




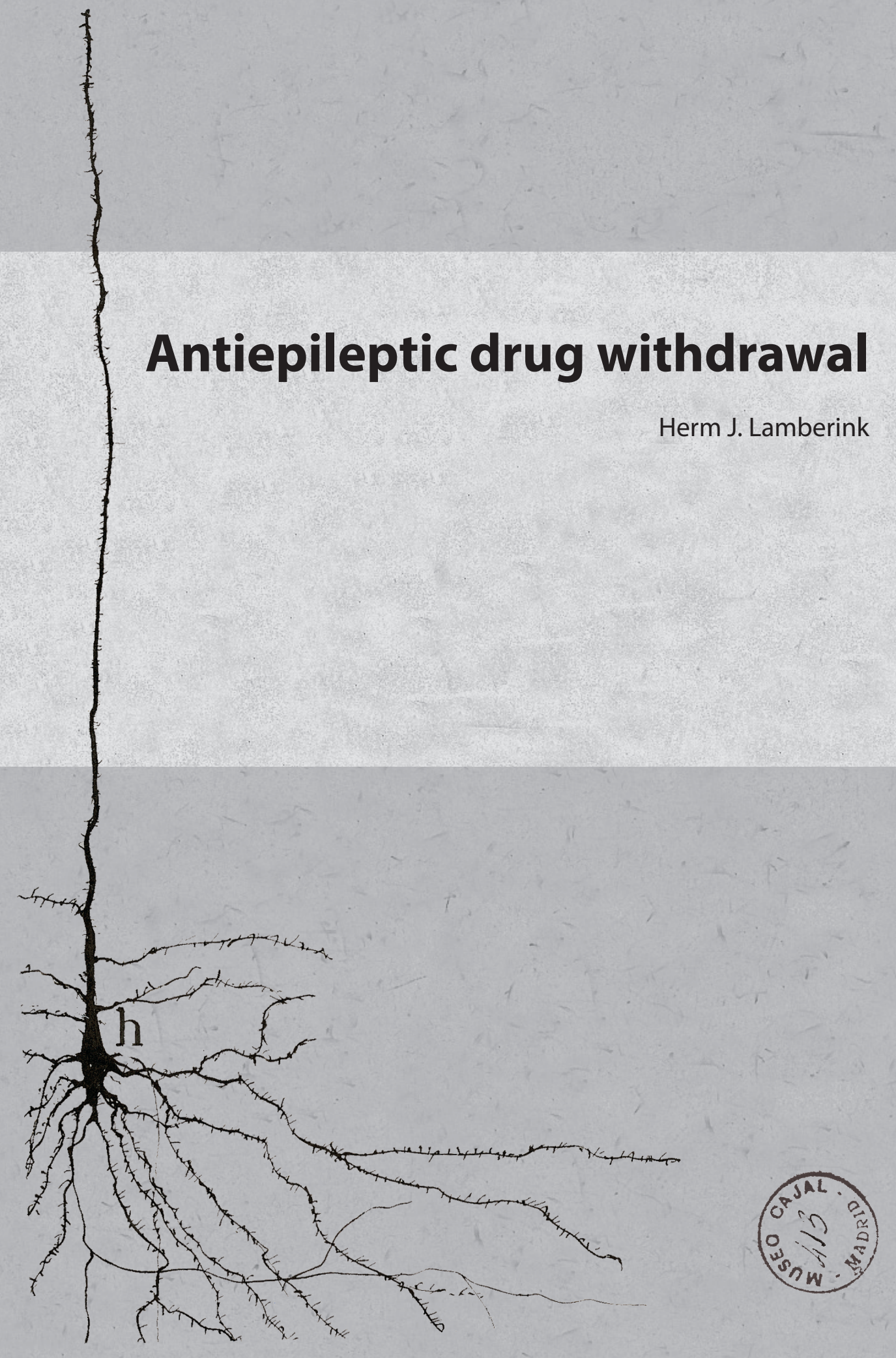


Antiepileptic drug withdrawal

Copyright $\odot$ Herm J. Lamberink

No part of this publication may be reproduced or transmitted in any form or by any means, without permission in writing from the author. The copyright of the articles that have been published or have been accepted for publication has been transferred to the respective journals.

ISBN:

978-90-393-7186-2

Cover design and layout: Herm Lamberink

Cover image:

Santiago Ramón y Cajal, drawing M413. Courtsy of the Cajal Institute, Cajal Legacy, Spanish National Research Council (CSIC), Madrid, Spain. Printed with permission.

Printing: Gildeprint BV

The research described in this thesis was supported by a grant of the Epilepsiefonds (08-10) 


\title{
Antiepileptic drug withdrawal
}

\author{
Het staken van anti-epileptica
}

(met een samenvatting in het Nederlands)

\section{Proefschrift}

ter verkrijging van de graad van doctor aan de

Universiteit Utrecht

op gezag van de

rector magnificus, prof.dr. H.R.B.M. Kummeling, ingevolge het besluit van het college voor promoties

in het openbaar te verdedigen op

dinsdag 15 oktober 2019 des middags te 2.30 uur

door

Herman Jan Lamberink

geboren op 30 november 1989

te Zwolle 


\title{
Promotor:
}

\author{
Prof. dr. K.P.J. Braun
}

\section{Copromotoren:}

Dr. W.M. Otte

Dr. C.P.W. Geleijns

Dit proefschrift werd mede mogelijk gemaakt met financiële steun van het Epilepsiefonds. 



\section{CONTENTS}

\section{General introduction}

Chapter $1 \quad$ Introduction, aim and outline

Part ONE

Chapter 2

Chapter 3

Chapter 4

\section{PART TWO}

Chapter 5

Chapter 6

\section{AED withdrawal in patients without surgery}

Antiepileptic drug withdrawal in medically and surgically treated patients: a meta- analysis of seizure recurrence and systematic review of its predictors.

Cumulative effects of antiepileptic drugs on intelligence in children with focal epilepsy.

Individualised prediction model of seizure recurrence and long-term outcomes after withdrawal of antiepileptic drugs in seizure-free patients: a systematic review and individual participant data meta-analysis.

\section{AED withdrawal after epilepsy surgery}

Changing profiles of pediatric epilepsy surgery candidates over time: A nationwide single-center experience from 1990 to 2011. multicentre European histopathology study of 9,147 patients who underwent epilepsy surgery. 
Chapter $7 \quad$ Cognitive consequences of early versus late antiepileptic drug withdrawal after pediatric epilepsy surgery, the TimeToStop (TTS) trial: study protocol for a randomized controlled trial.

Chapter 8 Why the TimeToStop trial failed to recruit: a survey on antiepileptic drug withdrawal after paediatric epilepsy surgery.

Chapter $9 \quad$ Individualized prediction of seizure relapse and outcomes following antiepileptic drug withdrawal after pediatric epilepsy surgery.

\section{General discussion}

Chapter $10 \quad$ Discussion

\section{Addendum}

References

Summary

Nederlandse samenvatting

List of publications

About the author

Dankwoord 

GENERAL INTRODUCTION 

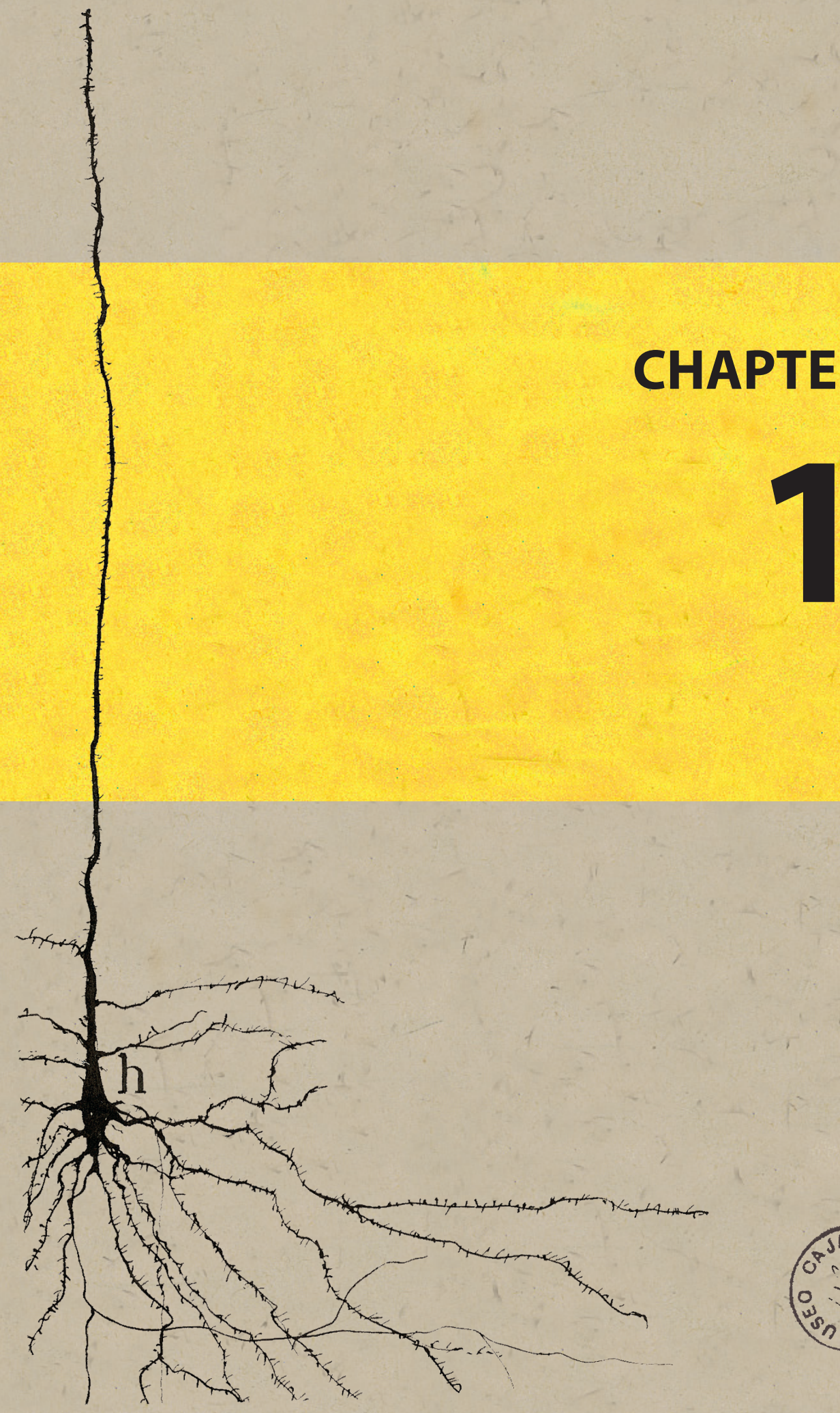


\section{Introduction, aim and outline}




\section{INTRODUCTION}

Epilepsy is a disorder that affects many people in the world. The exact number probably lies between 50 million (World Health Organization 2006) and 69 million people (Ngugi et al. 2010). With many treatment options available today, a large proportion of people can control, and be free from, their seizures. The most common treatment is medication, antiepileptic drugs (AEDs), that prevent seizures but do not cure epilepsy. Using AEDs for seizure prevention often comes at the cost of sedative and cognitive side effects. Moreover, even if seizures have not been present in somebody's life for years, the daily intake of the medication is a constant reminder of the presence of their disease. The person is free from seizures, but not from epilepsy. In contrast, if the medication can also be safely discontinued, and both seizure- and medication-freedom persist over ten years, they can be considered 'cured' (Fisher et al. 2014). When the medication has successfully prevented seizures for years, or when another treatment has taken away the assumed cause of epilepsy, the following questions arise: is it safe to stop treatment, and - if so - when should we start tapering? Stopping AED treatment might have many benefits, but it simultaneously increases the short-term risk of seizures. This dilemma is the background of all chapters following this introduction.

To provide context for these chapters, some basic principles about epilepsy, its causes, treatment options, and current knowledge on AED withdrawal will be outlined below.

\section{Definition of epilepsy}

Epilepsy can be defined as the experience of recurring seizures, or as a general predisposition of the brain toward generating seizures. In such a seizure, groups of neurons are temporarily and involuntarily firing simultaneously, resulting in transient loss of function related to the epileptic discharge in the brain. This pathological pattern of synchronous neuronal activity does not occur in a healthy brain. Clinically, seizures are divided into two types, based on the onset of the seizure being either focal or generalized (Fisher et al. 2017). In a focal seizure a local group of neurons are firing synchronously, and clinically the seizure can show symptoms related to that brain location. For example, a seizure in the left motor cortex may cause the right hand to twitch. When the whole brain is involved in a seizure -a generalized seizure- a person is not able to communicate, consciously act, and will not remember what happened during the seizure. 
The formal definition for the diagnosis of epilepsy is established by the International League Against Epilepsy (ILAE). The diagnosis can be made when a patient falls into one of the following three categories (Fisher et al. 2014):

1. At least two unprovoked (or reflex') seizures which occurred 24 hours apart from each other

2. One unprovoked (or reflex) seizure, and a chance of further seizures of at least $60 \%$

3. The diagnosis of a specific epilepsy syndrome ${ }^{2}$

When seizures are caused by an acute physical or chemical stimulus, like a head trauma or intoxication, they are called provoked seizures, which are explicitly excluded in the above definition of epilepsy.

\section{Etiology}

There are many different causes of epilepsy. In 2017 the ILAE has made the following distinction of six major categories of etiology (Scheffer et al. 2017): structural, genetic, infectious, metabolic, immune, and unknown. The structural epilepsies are caused by a lesion that can be seen on imaging or histopathology, such as a malformation of cortical development, a tumor, or a scar related to an old ischemic stroke (Scheffer et al. 2017). Genetic epilepsies are primarily caused by a genetic defect. A distinction should be made here between the clearly causal, usually mono-genetic defects causing epilepsy, and the many different types of genes which increase the risk of epilepsy but do not cause epilepsy by a single mutation (Helbig, Heinzen, and Mefford 2016). However, when a genetic defect is causing structural abnormalities, such as tubers or other malformations of cortical development, this will rather be classified as a structural epilepsy (Scheffer et al. 2017). Worldwide, the most common preventable group of etiologies are infectious diseases (Vezzani et al. 2016). Examples of infectious causes for epilepsy are neurocysticercosis, tuberculosis, HIV, and cerebral malaria. The metabolic epilepsies usually also have a genetic base, and awareness of this genetic defect could have major therapeutic consequences. An example of a metabolic epilepsy is pyridoxine-dependent epilepsy, in which seizures are controlled by a life-long supplementation of high-dose vitamin B6 (Stockler et al. 2011; Karnebeek et al. 2016). In immune related epilepsies the usual mechanism of disease

$1 \quad$ Reflex seizures: "The condition of recurrent reflex seizures, for instance in response to photic stimuli, represents provoked seizures that are defined as epilepsy. Even though the seizures are provoked, the tendency to respond repeatedly to such stimuli with seizures meets the conceptual definition of epilepsy, in that reflex epilepsies are associated with an enduring abnormal predisposition to have such seizures." (Fisher et al. 2014)

2 Epilepsy syndrome: "An epilepsy syndrome refers to a cluster of features incorporating seizure types, electro-encephalography (EEG), and imaging features that tend to occur together." (Scheffer et al. 2017) 
is that of auto-antibodies against a neuronal target, such as the NMDA receptor or GABA receptor (Lancaster and Dalmau 2012). Lastly, when the causal mechanism for the epilepsy remains unknown, it will be classified as such.

\section{Natural course of epilepsy}

When discussing the efficacy of treatment, it is important to know the natural course of a disease in the absence of treatment. This information can usually be taken from placebocontrolled trials where the placebo arm represents the untreated history of the disease. However, since AEDs have been available for over 100 years (phenobarbital was discovered in 1911 (Yasiry and Shorvon 2012) and bromide in the 1850's (Eadie 2012)) placebo-controlled mono-medication trials are not available nor deemed ethical. Therefore, knowledge on the natural history of epilepsy comes mainly from resource-poor settings where treatment is or was not available for many people (Beghi, Giussani, and Sander 2015). From these observational data it can be induced that $30 \%$ to $50 \%$ of people with epilepsy may eventually be free from seizures again, without any intervention (Beghi, Giussani, and Sander 2015). However, the fact that many people become seizure-free naturally is not enough to refrain from starting therapy, as each additional seizure can have detrimental effects. People in whom seizures are not successfully prevented by AEDs are namely more than twice as likely to die than the general population (Mohanraj et al. 2006; Trinka et al. 2013). Furthermore, refractory epilepsy is negatively associated with employment status (Marinas et al. 2011; Sillanpää, Haataja, and Shinnar 2004), quality of life (Baker et al. 1997; Sillanpää, Haataja, and Shinnar 2004), and social parameter such as being married, having children, or a driver's license (Sillanpää, Haataja, and Shinnar 2004). Lastly, continuing seizures have a negative impact on cognition (Berg et al. 2012; Holmes 2016). For all these reasons, initiating treatment is preferred above 'watchful waiting' in many patients once the diagnosis is established.

\section{Treatment options}

Epilepsy comes with several adjustments and restrictions to normal life. Beside avoiding situations that pose hazards like swimming or driving a car, other situations should be avoided because they could trigger seizures, such as sleep deprivation, alcohol, or parties with flashing lights. The first-line treatment to prevent seizures are AEDs (Glauser et al. 2006, 2013). These drugs are administered to almost all people with epilepsy: an Italian population-based study of 747 people with epilepsy showed that $98 \%$ of them had started AEDs (Giussani et al. 2017). When therapy fails and seizures keep recurring, the epilepsy is said to be medically refractory or drug-resistant. Drug-resistant epilepsy is defined by the ILAE as "failure of adequate trials of two tolerated and appropriately chosen and used AED schedules (whether as monotherapies or in combination) to achieve sustained seizure freedom" (Kwan et al. 2010). In the case of 
drug-resistant epilepsy, a small group of epilepsy patients in which the exact location of seizure-generating brain tissue can be identified can benefit from epilepsy surgery (Ryvlin, Cross, and Rheims 2014). For people with drug-resistant seizures who are not eligible for surgery, several other therapy strategies are available with the aim to reduce the seizure load and/or frequency, and sometimes to achieve seizure freedom. An example is the ketogenic diet, which reduces the availability of glucose to the brain and replaces it by ketones, an alternative source of energy for neurons and other body cells (D'Andrea Meira et al. 2019). Dietary therapy is promising, but the evidence for its efficacy is limited (Martin-McGill et al. 2018). Electrical stimulation of the vagal nerve is another therapy that is applied in refractory epilepsy, which has been proven to effectively reduce the seizure frequency in many different forms of epilepsy (Panebianco et al. 2015). Other methods of nerve and brain stimulation are employed to treat epilepsy also, with the goal of disrupting epileptogenic networks and/or seizure propagation (Schulze-Bonhage 2017). Lastly, immune-suppressive therapies have been suggested and are currently investigated, since the immune system is involved in some forms of epilepsy. In West syndrome for example, immune suppressive treatment, in combination with vigabatrin, is currently the most effective treatment (O'Callaghan et al. 2017). Similarly, patients with electrical status epilepticus during slow-wave sleep (ESES) often benefit from steroid treatment (van den Munckhof et al. 2015). In other epilepsies the efficacy of immune suppression is unknown (Mehta et al. 2015).

Despite all these available treatments, the question remains whether epilepsy can ever be considered 'cured'. In some patients epilepsy will resolve over time, with or without treatment. There will, however, always remain a low risk of seizures in these people, which is assumed to be higher than in the general population (Fisher et al. 2014). An ILAE task force therefore decided to use the term 'resolved' rather than 'cured' (Fisher et al. 2014). Epilepsy may be called resolved when ten years have passed without seizures, of which at least the last five years were without medication. The focus of our studies, described in this thesis, will be on the two most effective treatments in epilepsy, which will be addressed in more detail below: AEDs and epilepsy surgery.

\section{Antiepileptic drugs}

AEDs prevent seizures, but do not treat the underlying cause or disease responsible for the seizures. This was already noted by the English neurologist William Gowers in 1881.

"The treatment of epilepsy by bromide or other drugs is for the most part, as already stated, a question of arresting the fits, or, failing this, of producing the greatest possible diminution in their frequency and severity. The arrest of fits is of course not equivalent to the cure of the disease. In only too many cases, when the attacks 
have been arrested for a long time by treatment, they recur when that treatment is discontinued." (Gowers 1881)

Unfortunately, the name'antiepileptic drugs' suggests that these drugs actually treat epilepsy. Because this does not accurately describe their function, a better name for this class of drugs would be anticonvulsants or antiseizure drugs. For practical reasons the term 'antiepileptic' will still be used in this thesis, because this is the term commonly employed in the current literature.

AEDs act on various key elements in neurotransmission, preventing generation and spread of seizures (Rogawski and Löscher 2004). The most important drug targets are the neurotransmitter systems of gamma-aminobutyric acid (GABA) and glutamate, and the voltage-gated ion channels for sodium, potassium and calcium (Rogawski and Löscher 2004; Das, Dhanawat, and Shrivastava 2012). For many types of epilepsies, genetic defects in one of these molecular targets have been found, such as the SCN1A gene for a sodium channel subunit (Escayg and Goldin 2010; Helbig, Heinzen, and Mefford 2016). Recently, novel genetic correlates of epilepsy have been found, some of which are not yet targeted by any of the existing AEDs (The International League Against Epilepsy Consortium on Complex Epilepsies 2018). This new knowledge has created opportunities for the development of novel AEDs.

\section{Efficacy and side effects of AEDs}

Of all people newly diagnosed with epilepsy, approximately two out of three will become seizure free after starting AED treatment. The efficacy of treatment in new patients is known from a large observational study in Scotland where 1795 people newly diagnosed with epilepsy were followed up after initiation of AED therapy (Z. Chen et al. 2018). At the end of the study 1144 people (64\%) were free from seizures for at least 12 months after trying between one and eleven AEDs, of which most $(1028 / 1795,57 \%)$ became seizure free with the first or second drug. Because many people did not try a second or third AED, the probability of seizure freedom after three or more drugs is probably higher than reported.

Because AEDs either promote inhibitory systems or downregulate excitatory systems, therapy may also have significant consequences for normal functioning of a patient. AEDs have multiple side effects, many of which are the result of a direct effect on the modulation of neuronal excitability. The most commonly reported side effects are those that involve the central nervous system (Perucca and Gilliam 2012). Most AEDs share a sedative and cognitive adverse effect, which increases with higher doses, and therefore the most common complaints are drowsiness, imbalance, fatigue, concentration and memory problems (Perucca and Gilliam 2012; Mula and Trimble 2009). In children specifically, the drugs that are predominantly related to these side effects are phenobarbital, phenytoin, topiramate and zonisamide (Aldenkamp 
et al. 2016). Behavioral side effects such as aggression, mood or anxiety issues were noted for the following AEDs: phenobarbital, valproic acid, gabapentin, topiramate, levetiracetam, and zonisamide (Aldenkamp et al. 2016). Beside these direct and dose-dependent effects, AEDs may have multiple other adverse effects. There are rare but serious effects such as severe mucocutaneous reactions or acute hepatotoxic effects, which subside with prompt cessation of the AED but have high morbidity and mortality; problems with decreased bone mineral density or weight gain or loss; teratogenic effects of AEDs during pregnancy; and interactions between drugs that may interfere with efficacy (Perucca and Gilliam 2012). Regarding the effects during pregnancy, the risk for the child is quite high for specific drugs, with $10 \%$ risk of birth defects for valproic acid use, and between $4 \%$ and $7 \%$ for topiramate, carbamazepine, phenytoin and phenobarbital (Tomson et al. 2018). For valproic acid the European Medicines Agency has now obliged a pregnancy prevention program for women who are prescribed the drug (European Medicines Agency 2018). Although teratogenicity is the most obvious and detrimental effect of AEDs during pregnancy, more subtle effects have been identified including a relation with lower IQ with valproate (Bromley and Baker 2017) and primidone (Koch et al. 1999) and an increased risk of autistic spectrum disorder with valproic acid (Christensen et al. 2013).

In two studies from 1997 and 2001, 88\% of people using AEDs experienced at least one side effect (Perucca et al. 2009)including the Adverse Event Profile (AEP, and an average patient may report six or seven different side effects (Perucca et al. 2009)including the Adverse Event Profile (AEP. However, in those studies carbamazepine and phenytoin were among the most commonly used drugs, which are currently prescribed less often, and are partly replaced by more modern drugs like levetiracetam, lamotrigine and oxcarbazepine (Z. Chen et al. 2018). These more modern drugs are believed to have a better side effect profile, although the evidence for this is circumstantial (Mula and Trimble 2009). In children, levetiracetam and lamotrigine have sometimes even been associated with an improvement of cognitive function (Aldenkamp et al. 2016). It may therefore be that at time of writing less people are experiencing cognitive side effects than presented in above studies.

\section{Epilepsy surgery}

In some patients with focal - and presumed structural - epilepsy it may be possible to surgically remove the epilepsy-generating source, also referred to as the seizure onset zone. To determine whether someone is eligible for surgery, there should be reasonable confidence that (1) seizures are generated in (a) resectable area(s), and (2) that there are no major functions located in the resection area, such as speech or language-associated cortex (Ryvlin, Cross, and Rheims 2014). This is ascertained by combining information from different modalities. First, the seizure semiology, magnetic resonance imaging (MRI) and EEG may give information on the seizure onset zone (Ryvlin, Cross, and Rheims 2014). Second, more advanced imaging techniques 
like magnetoencephalography (MEG), ictal single photon emission computed tomography (SPECT), and interictal fludeoxyglucose-positron emission tomography (FDG-PET) may give more insight into the epileptogenic zone (Ryvlin, Cross, and Rheims 2014). Lastly, intracranial EEGs using a grid of electrodes on the cortical surface or depth electrodes can be used when exact source localization with previous methods was not possible, for example when the seizure onset zone is presumed to lie deeper in the brain tissue (Ryvlin, Cross, and Rheims 2014; Kovac et al. 2017). A community-based follow-up study of children with newly-diagnosed epilepsy suggested that approximately $25 \%$ (132/518) of children were pharmacoresistant, of whom only $10 \%$ (54/518) were referred for evaluation at a comprehensive epilepsy surgery center (Berg et al. 2009). In the end, 22/518 children underwent epilepsy surgery (4\%) (Berg et al. 2009). Other studies indicated that epilepsy surgery is still under-utilized (De Flon et al. 2010), also in children (Pestana-Knight et al. 2015) and adults specifically (Uijl et al. 2012).

Epilepsy surgery is accepted as a very effective treatment in well-selected patients, although neurosurgery has side-effects and risks. Firstly, there may be anticipated neurological deficits that are usually accepted as a small price to pay for potential seizure freedom. These deficits are related to the resection area, most commonly a partial visual field defect after temporal lobe surgery (Steensberg et al. 2018). Secondly, complications regarding surgery that bear no consequences for long-term seizure or functional outcomes can occur, such as an infection or leakage of cerebrospinal fluid requiring a second surgery. These post-surgical issues happen in approximately $5 \%$ of the patients (Hader et al. 2013). Lastly, the most serious complications are unexpected neurological deficits and surgery-related death, the latter are reported in up to $0.6 \%$ of the cases (Hader et al. 2013; Bjellvi et al. 2015).

The reason these rare but serious complications are risked, is the prospect of becoming seizure free, as the average chance to become free from seizures is $65 \%$ (West et al. 2015). This is in high contrast to the slim chance of becoming seizure free after receiving the diagnosis of drugresistant epilepsy. A recent randomized trial in pediatric epilepsy surgery candidates revealed - as expected - a seizure-freedom rate of $77 \%$ one year after surgery compared to $7 \%$ in the control group who continued medical treatment (Dwivedi et al. 2017). 


\section{ANTIEPILEPTIC DRUG WITHDRAWAL}

Once patients become seizure-free the inevitable question arises whether -or whenmedication can be safely stopped, and in whom. Not every person with epilepsy in remission will attempt to stop AED treatment. In fact, a recent population-based study showed that $64 \%$ of AEDs were never withdrawn (Giussani et al. 2017). Another $15 \%$ of drugs were withdrawn for reasons of ineffectiveness and supposedly replaced by other AEDs. Overall, only $11 \%$ of AEDs were withdrawn for the reason of expected 'terminal remission' (Giussani et al. 2017). Comparing this number to the fact that two out of three people become seizure free thanks to $A E D s$, it seems that most people continue medication despite being seizure free. The decision to start AED withdrawal will depend on the personal convictions and risk-seeking or -avoiding preferences of the patient, the potential personal consequences of a seizure and the experience of negative effects of the drugs, among others. In addition, the experience and convictions of the physician may have an influence on how many of her or his patients ultimately withdraw AEDs. In practice, the potential risks are contrasted against the expected benefit for every individual patient before deciding to withdraw or continue AED treatment. Below, these risks and benefits are discussed for those who become seizure free on AEDs, followed by a section on the situation after epilepsy surgery.

\section{Risks of AED withdrawal}

When AEDs are discontinued approximately $60 \%$ to $70 \%$ of people remain free from seizures. In the remaining patients seizures return and medication needs to be restarted (Braun and Schmidt 2014). A first reason to continue AEDs would be a known higher-than-average risk of seizure recurrence, for example in patients with juvenile myoclonic epilepsy (Höfler et al. 2014; Syvertsen et al. 2019; Healy et al. 2018). Another argument to be careful with AED withdrawal is the unknown course of epilepsy after seizure recurrence. Approximately $80 \%$ of people who experience recurrent seizures will be able to control them again by restarting their AEDs, although this takes longer than six months in half of them and up to five to twelve years for some (Schmidt and Löscher 2005). One in five people will continue to experience seizures despite restarting AED treatment (Schmidt and Löscher 2005). Although there is no evidence that refractory epilepsy after an AED withdrawal attempt is causally related to AED withdrawal (Braun and Schmidt 2014), it is a potential outcome that should be discussed with the person considering AED withdrawal.

In the case of pregnancy, it seems intuitive to attempt AED withdrawal because the drugs may harm the fetus. This is however not the case, because seizures during pregnancy carry their own risk for mother and child. Especially generalized tonic-clonic seizures are associated with 
increased maternal and infant death (Edey, Moran, and Nashef 2014), as well as with low birth weight and preterm delivery (Y.-H. Chen et al. 2009).

It should be stressed that continuation of therapy does not mean there is no risk of seizure recurrence. Results from the two randomized trials on the topic illustrate this clearly. Twelve months after randomization, the control arm still experienced $7 \%$ seizure recurrences compared to $15 \%$ in the AED withdrawal arm in the Akershus randomized trial (Lossius et al. 2008). At 24 months, the MRC trial showed a similar difference: $22 \%$ recurrences for the control group, compared to $41 \%$ in the AED withdrawal arm (MRC AED withdrawal study group 1991). From these trials it can be concluded that compared to AED withdrawal, the risk of seizures is approximately half for those who continue treatment, at least in the first two years.

\section{Benefits of AED withdrawal}

On the other hand there are good reasons to consider withdrawal of medication. To start, it is the only way to achieve complete remission according to official definitions (Fisher et al. 2014). Aspects to consider are the previously mentioned cognitive and behavioral side effects of AEDs (Aldenkamp et al. 2016; Mula and Trimble 2009) and the quality of life of the person with epilepsy (Sillanpää, Haataja, and Shinnar 2004). In girls and women, the teratogenic potential in relation to future pregnancy is another consideration. Lastly, chronic side effects of drugs such as permanent visual field defects by vigabatrin or changes in body weight (Perucca and Gilliam 2012), drug-to-drug interactions in the case of for example oral contraceptive drugs (Schwenkhagen and Stodieck 2008), and the societal costs of overtreatment are reasons to consider AED withdrawal.

Some of these potential benefits have been tested in the earlier mentioned randomized trials. Results from the Akershus randomized trial have shown, for example, that AED withdrawal improves cognition (Lossius et al. 2008). This trial showed statistically significant differences between those who withdrew and control participants, regarding processing speed to perform cognitive tasks (Hessen et al. 2006), word fluency (Hessen et al. 2006), executive function (Hessen, Lossius, and Gjerstad 2009), and verbal memory (Hessen, Lossius, and Gjerstad 2011). The research group of the MRC randomized trial studied psychosocial outcomes of AED withdrawal, and observed that randomization to discontinuation was related to increased selfesteem at two years (Jacoby, Johnson, and Chadwick 1992). Similarly, more people described their own health as excellent or good two years after randomization, compared to those who continued medication (Jacoby, Johnson, and Chadwick 1992). When a person had experienced a seizure recurrence or was still taking AEDs at two years, they were more likely to be worried about epilepsy, to feel restricted in social activities, and to indicate they were kept from doing certain things because of the epilepsy (Jacoby, Johnson, and Chadwick 1992). 


\section{AED withdrawal after epilepsy surgery}

After epilepsy surgery the situation is slightly different, though many of the above-mentioned considerations apply here as well. When surgery has been seemingly successful and no seizures occurred afterward, it is very likely that the brain does not anymore qualify as having a "general predisposition toward generating seizures". However, as long as AED treatment is continued it will not be known whether there is any residual seizure activity. In the first postoperative months it is unknown whether the ictogenic focus was completely removed, and many cases of surgical failure will present themselves during this time with seizures regardless of AED treatment. By waiting for some time, a selection of potential candidates for AED withdrawal can be made. Arguments to continue AED treatment for longer periods, say for years, are harder to substantiate. The fact that AED withdrawal temporarily increases the risk of seizure recurrence is only partially valid - if surgery was successful, a person should not have epilepsy anymore and there is no risk of seizures. Only in the cases where there is any residual epileptogenic network the risk of seizures will increase after AED withdrawal. A study by Boshuisen and colleagues (Boshuisen et al. 2012)investigated this: early withdrawal after pediatric epilepsy surgery did increase the risk for seizure recurrence, but there was no relation with long-term seizure freedom. There is no reason to believe that the situation is different for the adult population. A retrospective study in adults after temporal lobe surgery confirms that long-term seizure freedom rates are similar between those discontinued AEDs early or late after surgery, although people with a seizure recurrence during AED tapering had lower chances of seizure freedom compared to seizure recurrences occurring after complete discontinuation (Yardi et al. 2014). Again, there is no evidence that this was causally related to the timing of AED withdrawal. Overall, early withdrawal unmasked surgical failure and prevents overtreatment in the remaining surgical success cases.

The cognitive advantages of AED withdrawal after epilepsy surgery on the other hand are becoming clear. In children, stopping AEDs increases IQ by 6 points on average although there is large variation between patients (Boshuisen et al. 2015). This positive association between cessation of AEDs and improvement in IQ was also found by another group (Skirrow et al. 2011). AED reduction was also correlated to improved psychomotor speed (Van Schooneveld et al. 2013) and executive function (Helmstaedter, Elger, and Witt 2016). A recent study found that surgery, compared to no surgery, more often leads to significant improvement in IQ, but this study failed to show an effect of AED withdrawal on postoperative IQ (Skirrow et al. 2019). Most of the mentioned studies are quite small, and variation between patients is very large with significant decline in IQ scores for some. The average patient however, benefits cognitively from surgery and from postsurgical AED withdrawal. 


\section{Knowledge gap}

The sections above indicate that much of the risks and benefits of AED withdrawal are already known, and that there are many aspects a physician can use in counselling his or her patient on the choice to withdraw or continue. The information available however, is not complete, and there are important questions that remain. To estimate the risk for an individual patient, it is important to know which factors are most strongly related to elevated or decreased risks. Currently it is not known which factors should be used, because many different studies have indicated different determinants. More importantly, there is no tool available to calculate the risk of seizure recurrence for the individual patient. Furthermore, previous research on the cognitive benefits of AED withdrawal has sparked new questions to be studied. The longterm effects of AEDs on the development and cognition of children for example is not known. Also, all knowledge on the postoperative cognitive benefits of AED withdrawal are based on retrospective data. Will the cognitive benefits be as clear when studied in a prospective and randomized manner? 


\section{AIM OF THESIS}

The overall aim of this thesis was to quantify the risks and benefits of AED withdrawal in more detail for patients who become seizure-free after medical treatment and for patients who reach seizure-freedom after epilepsy surgery.

The specific objectives were:

- To calculate cumulative risks of seizure relapse after AED withdrawal in medically- and surgically treated epilepsy patients who became seizure-free

- To investigate potential long-term negative effects of AEDs on the cognition of children

- To develop tools that enable the prediction of individualized risks of seizure recurrence after AED withdrawal in seizure-free subjects

- To explore differences in AED withdrawal policies in paediatric epilepsy surgery over time and between European epilepsy surgery centers

- To study cognitive benefits of early AED withdrawal after pediatric epilepsy surgery 


\section{OUTLINE OF THESIS}

\section{Part I. AED withdrawal in patients without surgery.}

The first part of the thesis starts with an overview of the literature in Chapter 2. Previous publications are studied through a systematic review of the existing literature. A meta-analysis of the average recurrence risks is presented, as well as an overview of previously reported risk factors. Chapter $\mathbf{3}$ addresses a more fundamental question regarding the potential influence of AEDs on brain development in children, namely: are there reasons to believe that AEDs might actually harm long-term cognitive development? In Chapter $\mathbf{4}$ a new tool is presented to predict individualized risks of relapse in people with epilepsy who have become seizure free during AED therapy.

\section{Part II. AED withdrawal after epilepsy surgery.}

The first two chapters in this section mainly focus on surgery outcomes and medication withdrawal policies. Chapter 5 presents seizure-outcomes and drug policies during the first 22 years of the nationwide Dutch pediatric epilepsy surgery program. Chapter $\mathbf{6}$ addresses these issues in an uniquely large cohort of 9047 operated patients from 37 European epilepsy surgery centers. Next, to study the potential cognitive benefits of early versus late postoperative AED withdrawal in children, a randomized clinical trial was designed and initiated, of which Chapter $\mathbf{7}$ shows the trial protocol. Chapter $\mathbf{8}$ continues to discuss the challenges in patient recruitment for that trial, and presents the results of a survey among pediatric epilepsy surgery specialists. The survey focuses on current practices related to the timing of postoperative AED withdrawal. Chapter 9 is the counterpart of chapter 4, this time presenting a prediction tool to calculate individualized risks of seizure relapse after postoperative AED withdrawal in children.

Lastly, the discussion Chapter $\mathbf{1 0}$ takes the meta-view to assess what this thesis has added to our understanding of AED withdrawal. The findings will be placed in a broader context and directions for future research will be discussed. 


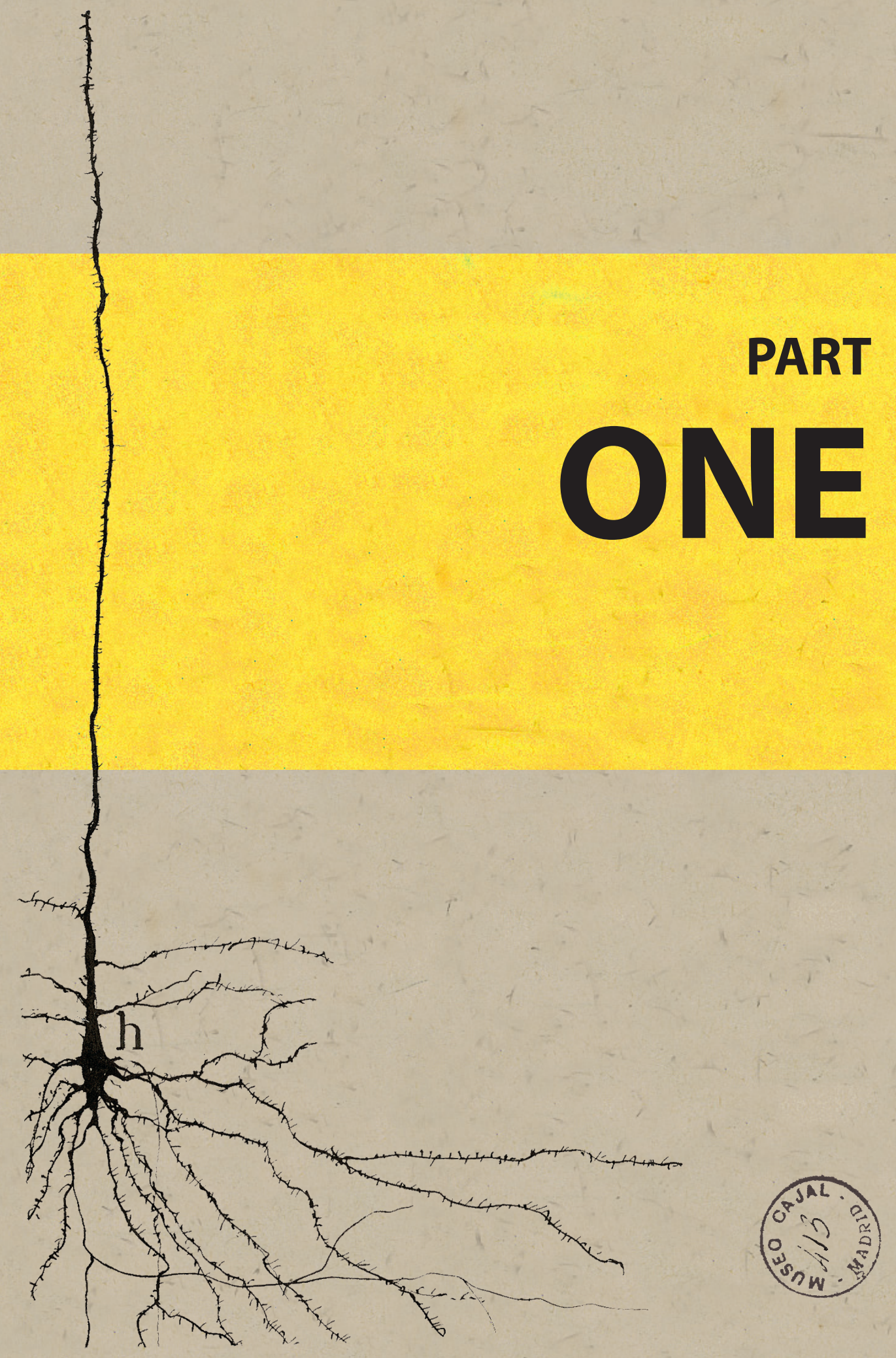




\section{AED WITHDRAWAL \\ IN PATIENTS \\ WITHOUT SURGERY}




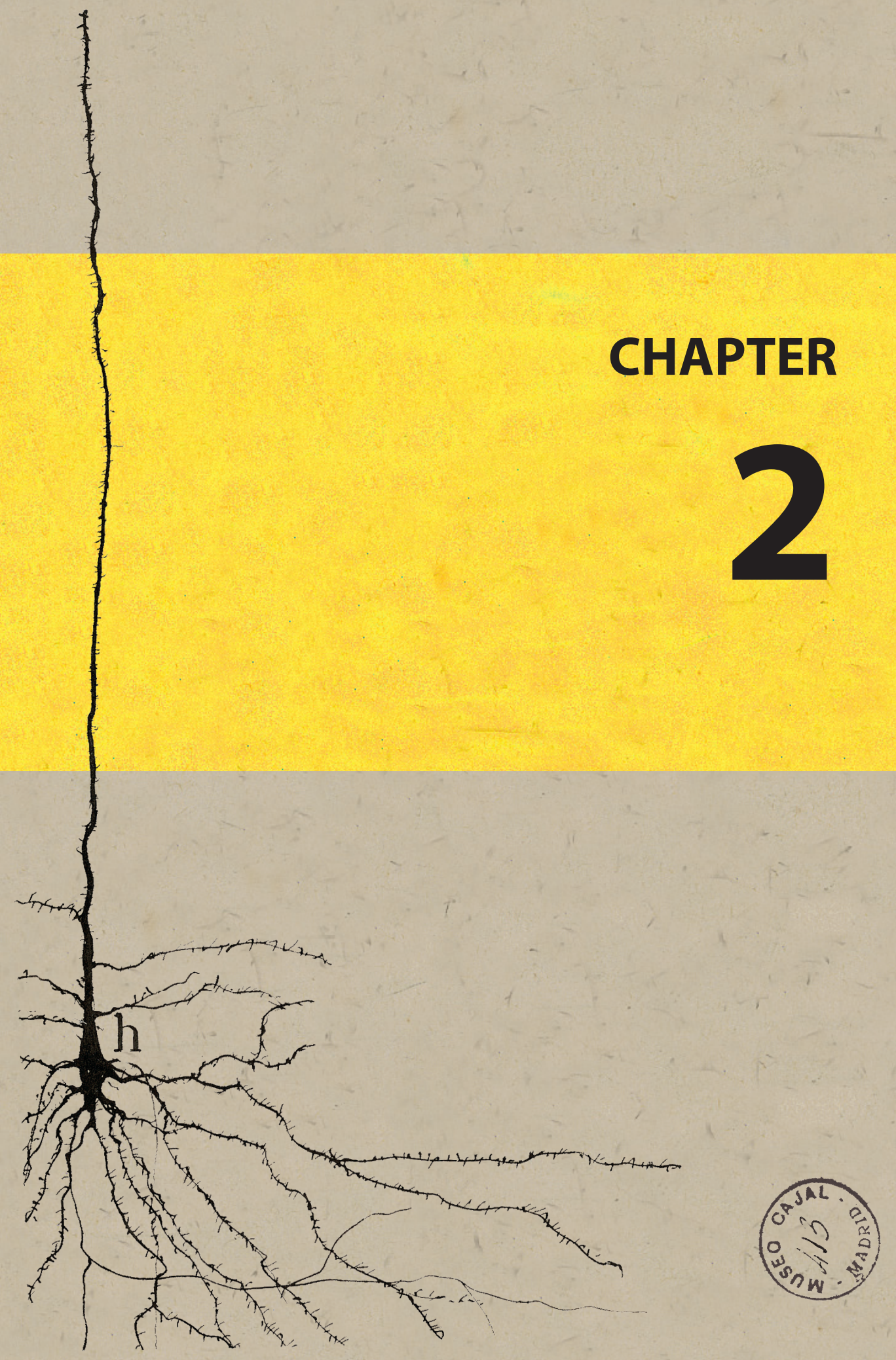




\section{Antiepileptic drug withdrawal in medically and surgically treated patients: a meta-analysis of seizure recurrence and systematic review of its predictors}

Herm J. Lamberink

Willem M. Otte

Karin Geleijns

Kees P.J. Braun

Epileptic Disorders. 2015;17(3):211-28 


\begin{abstract}
Aim: Many seizure-free patients consider withdrawal of antiepileptic drugs, both when seizure control is achieved by medication alone, or once they became seizure-free following epilepsy surgery. The risk of recurrence is consequently of very important prognostic value. However, estimations of recurrence risks are outdated for both populations. In addition, although many publications have reported predictors of seizure relapse, no comprehensive overview of prognostic factors is available.
\end{abstract}

Methods: A systematic review of the databases of PubMed and EMBASE was conducted, identifying articles on antiepileptic drug withdrawal in patient cohorts. Recurrence risk metaanalyses were performed for both populations at one, two, three to four, and five or more years of follow-up. Within the selected articles, studies presenting multivariable analysis of predictors were identified; all studied predictors were listed, as well as all significant independent predictors. The quality of separate analyses of predictors was assessed.

Results: There was no significant difference of long-term cumulative recurrence risk between surgical and medication-only populations, with respectively $29 \%$ and 34\% recurrences. In medication-only treated patients, 25 factors have been reported as significant independent predictors; 12 have been reported in surgical cohorts. The quality of most analyses of predictors was low to moderate. No predictor was consistently found among all analyses, and for most predictors, study results were contradictory.

Conclusion: No consistent set of predictors could be identified because a large number of variables have been identified in the literature, many studies reported contradicting results, study populations varied considerably, and the quality of the original studies was often low. Meta-analysis of individual participant data is necessary, because it allows for (1) correction for differences in follow-up duration between subjects and studies, (2) a study of interaction effects, (3) calculation of more accurate estimates valid across several populations, and (4) the assessment of each predictor's effect size. 


\section{Introduction}

Although antiepileptic drugs (AEDs) provide seizure freedom for the majority of people with epilepsy, nine out of ten patients experience at least one of the wide range of possible adverse effects (Perucca et al. 2009)including the Adverse Event Profile (AEP. A long-term follow-up study of childhood epilepsy showed that patients who became completely AED-free had a higher quality of life (QOL) than those continuing AED treatment, independently of being in remission or not (Sillanpää, Haataja, and Shinnar 2004). An important consideration for patients who become seizure-free, following either medical or surgical treatment, is whether or not an attempt should be made to stop AED treatment, with the risk of seizure recurrence. The significance of this decision is supported by the many reviews written on the subject (Berg and Shinnar 1994; Shlomo Shinnar and Berg 1995; Buna 1998; Greenwood and Tennison 1999; Alberto Verrotti et al. 2003; Luigi M Specchio and Beghi 2004; Shih and Ochoa 2009; Beghi and Schmidt 2013; Braun and Schmidt 2014). While the benefits of stopping AED treatment are clear, safety is a much debated issue. Previous meta-analyses have addressed seizure recurrence risks of AED discontinuation in medically treated (Berg and Shinnar 1994) and surgically treated patients (Ladino, Hernández-Ronquillo, and Téllez-Zenteno 2014). In the first study, cumulative recurrence rates at one and two years following start of withdrawal were $25 \%$ and $29 \%$, respectively. Relapse rates at later time points were not provided. In the pooled analysis of postoperative withdrawal studies, 708 of 2,901 patients $(24.4 \%)$ who withdrew AEDs had a seizure recurrence (Ladino, Hernández-Ronquillo, and Téllez-Zenteno 2014). Followup duration, however, was not accounted for. These studies did not systematically review the available evidence on predictors of seizure relapse. The independent predictive value of many clinical variables that have previously been reported to relate to seizure relapse remains debated, since studies have often revealed opposing results. When reviewing the available literature, the selection of studies referred to will therefore determine the conclusions reached by the reviewer. As an example, the predictive value of an EEG evaluation before the start of AED withdrawal is acknowledged by most authors but questioned in two articles (Alberto Verrotti et al. 2003; Luigi M Specchio and Beghi 2004).

The first objective of this study was to systematically review the literature and provide an updated meta-analysis of all available data in order to compare recurrence rates at different time intervals, including longer-term follow-up, after the start of AED reduction between two distinct populations: patients who became seizure-free with AEDs only (medically treated) and those who reached seizure freedom after epilepsy surgery (surgically treated). Second, we present an overview of possible predictors of seizure recurrence after AED withdrawal in both populations, as a first step towards an evidence-based estimation of recurrence risk in the individual patient. 


\section{Methods}

Study selection

Studies were selected based on two steps: first, articles were selected for meta-analysis of recurrence rates, after which all included articles were screened for the analysis of predictors of seizure outcome. To be eligible for inclusion in the meta-analysis of recurrence risks, manuscripts were required to be an original English full-text publication reporting a population of seizurefree epilepsy patients who attempted AED withdrawal. Both populations of medically treated and surgically treated patients were included (but analysed separately). The outcome of interest was seizure recurrence during or after AED withdrawal. Both retrospective and prospective observational studies were included, as well as randomized-controlled trials (RCTs). Excluded were case series and studies with $<20$ patients, cohorts that reinstituted AED therapy after an abnormal EEG (without having had a prior relapse), and publications on AED withdrawal in acute symptomatic seizures, neonatal seizures, or other populations that included patients not conforming to the 2014 ILAE definition of epilepsy (Fisher et al. 2014) (which includes the 1993 definition of at least two unprovoked seizures occurring 24 hours apart (International League Against Epilepsy 1993)).

The databases of PubMed and EMBASE were used until November 6, 2014; the full search string can be found in appendix 1. Duplicates were removed, after which two independent researchers ( $\mathrm{HJL}$ and $\mathrm{KG}$ ) screened the articles for eligibility based on above-mentioned criteria. All eligible articles were included in the meta-analysis, reference lists were checked for additional articles missed in the electronic search, and the articles were subsequently screened for the presence of multivariable analysis of risk factors for recurrence for inclusion in the systematic review.

\section{Data collection}

Full-text articles were screened by the first author to extract information on the following study characteristics and variables of interest: authors; publication year; geographical location; setting; publication type and in case of RCTs, the treatment and control groups; treatment (surgical versus medical); inclusion and exclusion criteria; number of participants; number of recurrences at one, two, three, four, and five or more years when available; duration of followup after start withdrawal (and, in some studies after surgery); predictors of seizure recurrence; potential for bias (see below); number of prognostic factors studied; and number and type of prognostic factors that were found to be significantly related to relapse.

Summary measures and risk of bias

A single group meta-analysis on recurrence risk after AED withdrawal was performed for both populations with different follow-up durations. Because less than half of studies on medical cohorts, and less than a third of studies on surgical cohorts, reported information on three- or four-year recurrence rates separately, these two follow-up times were combined, and when 
both were provided, the four-year recurrence rate was used. Similarly, the recurrence risks of all studies with follow-up of five or more years were averaged. To correct for differences in study sample size, meta-analysis was performed on the logit of the proportion of recurrence (logit=log[p/1-p], with variance[logit] $=[1 /$ number of recurrences] $+[1 /$ number of nonrecurrences], where $p$ equals the proportion of recurrences), according to Sutton et al. (Sutton et al. 2000). To correct for potential heterogeneity between studies, the meta-analysis was performed with a linear random effects model and additional Knapp and Hartung adjustments to obtain more accurate confidence intervals (Knapp and Hartung 2003). Meta-analysis summary estimates and corresponding $95 \%$ confidence intervals were back-transformed from logits to proportions.

Heterogeneity between studies was assessed with the 12 statistic according to Higgins and Thompson, where values between $50 \%$ and $75 \%$ are considered measures of moderate heterogeneity, and values $>75 \%$ high heterogeneity (Higgins and Thompson 2002). Statistical analyses were performed with R version 3.1.2 (R Core Team 2014), using packages "metaphor" and "boot".

In the surgical cohorts, the main meta-analysis included only those studies reporting follow-up after initiation of withdrawal (six studies). Studies that only reported follow-up relapse rates in relation to duration after surgery (ten studies) were excluded from the primary analysis, because the interval between surgery and AED withdrawal varied between subjects and studies. An additional analysis on studies that reported follow-up after surgery is shown as supplementary information.

Considering the systematic review of predictors of relapse, we compared, for each possible predictor, the number of studies that reported a significant correlation with relapse with the total number of studies that included the variable in the analysis. The possibility to metaanalyse the separate prognostic factors was evaluated, but many articles did not present data on non-significant predictors, resulting in a large publication bias rendering any result from meta-analysis unusable for solid conclusions. Therefore, the choice was made to perform a descriptive systematic review instead.

Quality appraisal and assessment of risk of bias of each study was performed with the Quality in Prognosis Studies (QUIPS) method proposed by Hayden and colleagues (Hayden, Coté, and Bombardier 2006). Six separate items are scored in this assessment: potential for bias in (1) study participation, (2) study attrition (loss to follow-up), (3) measurement of prognostic factors, (4) measurement of outcome, (5) measurement of, and accounting for, confounders, and (6) analysis and reporting. Since this is a descriptive systematic review of prognostic factors, no studies were excluded based on quality assessment. 


\section{Results}

From 2,588 articles identified, 61 articles were included in the meta-analysis, of which 45 (7,082 patients) concerned medically treated patients (Emerson et al. 1981; Holowach-Thurston et al. 1982; Shlomo Shinnar et al. 1985; Overweg et al. 1987; Arts et al. 1988; Callaghan, Garrett, and Timothy Goggin 1988; Alvarez 1989; Ehrhardt and Forsythe 1989; Matricardi, Brinciotti, and Benedetti 1989; MRC AED withdrawal study group 1991; Gherpelli et al. 1992; Galimberti et al. 1993; S Shinnar et al. 1994; Tennison et al. 1994; Uesugi et al. 1994; Delgado et al. 1996; Dooley et al. 1996; Tinuper et al. 1996; Braathen and Melander 1997; Caviedes and Herranz 1998; Marcus 1998; Altunbasak et al. 1999; Gebremariam, Mengesha, and Enqusilassie 1999; Alberto Verrotti, Morresi, Basciani, et al. 2000; Alberto Verrotti, Morresi, Cutarella, et al. 2000; Bouma, Peters, and Brouwer 2002; Lamdhade and Taori 2002; L M Specchio et al. 2002; Cardoso, Cendes, and Guerreiro 2003; Ohta et al. 2004; Camfield and Camfield 2005; Geerts et al. 2005; Serra, Montenegro, and Guerreiro 2005; Sillanpää and Schmidt 2006; Lossius et al. 2008; Olmez et al. 2009; Ramos-Lizana et al. 2010; Vurucu et al. 2010; Afshari and Moradian 2012; Pavlović, Jović, and Pekmezović 2012; A Verrotti et al. 2012; Su et al. 2013; Yang et al. 2013; Gafoor, Saifudheen, and Jose 2014; Li et al. 2014) and 16 (2,441 patients) surgical cohorts (Murro et al. 1991; Schiller et al. 2000; Griffin et al. 2004; Kim et al. 2005; Berg et al. 2006; Al-Kaylani et al. 2007; Sinclair et al. 2007; Lachhwani et al. 2008; Lee et al. 2008; Kerling et al. 2009; Park et al. 2010; Rathore et al. 2011; Boshuisen et al. 2012; Menon et al. 2012; Zeng et al. 2012; Yardi et al. 2014) (see figure 1 for full flow chart). A multivariable analysis of prognostic variables was performed in 27 studies on medically treated patients and in eight studies on surgical cohorts.

Meta-analysis of recurrence risk (table 1, figure 2)

Meta-analysis was performed on different subsets of articles, depending on the availability of data for the specific duration of follow-up (e.g. 36 medical cohorts with one-year followup data, see table 1). In medically treated patients, the cumulative recurrence rate climbed from $22 \%$ (95\% confidence interval [CI] 19\%-26\%) at one year, to $28 \%$ (24\%-32\%) at two years, and $34 \%(28 \%-40 \%)$ at three or four years. At five or more years of follow-up, the cumulative recurrence rate taken from 19 articles was lower, with an average of 27\% (23\%-32\%). Subgroup analysis of articles providing information on recurrences both at three to four years and at five or more years of follow-up, showed that recurrences after five years only occurred in less than $1 \%$ (Supplementary Table 1). The heterogeneity between studies was high, with $\mathrm{I}^{2}$ between $87 \%$ and $92 \%$ at different follow-up durations. 


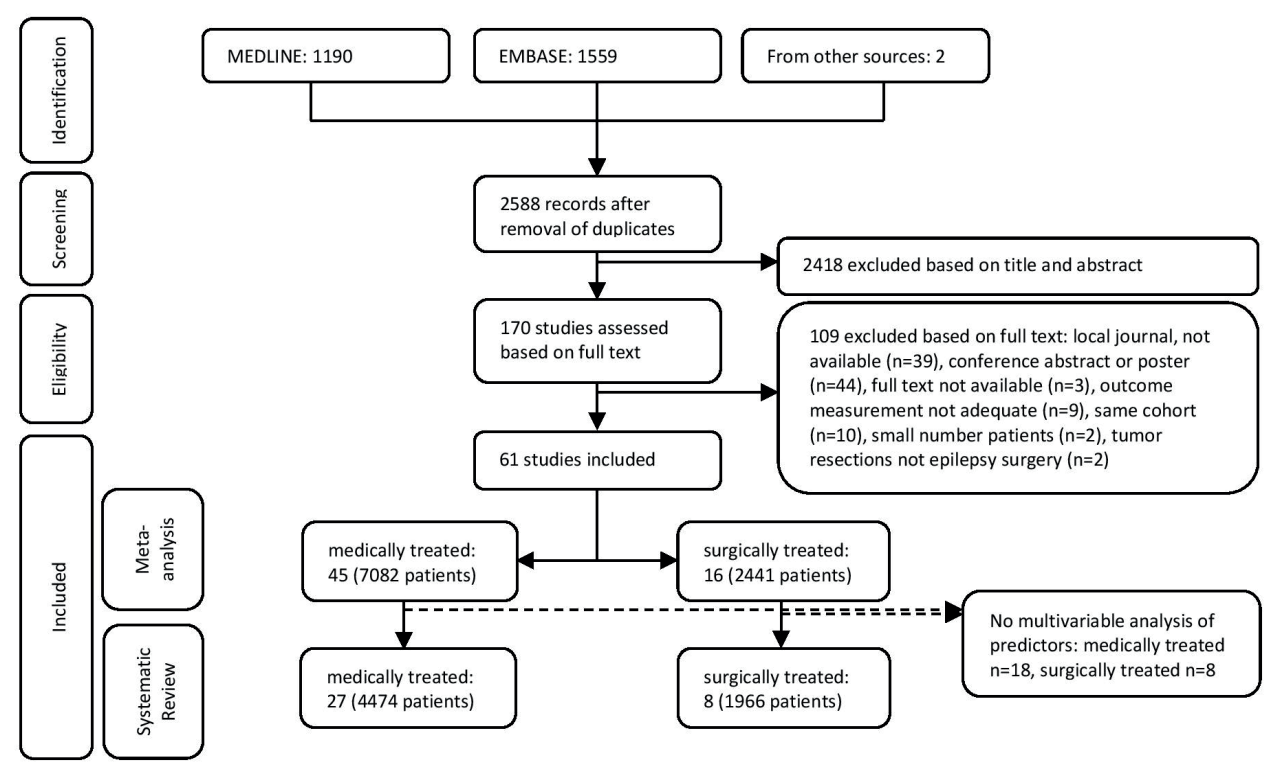

Figure 1 | Flow-chart of selection of publications.

Table 1 | Meta-analysis of recurrence risk after AED withdrawal. Separate meta-analyses were performed for the different follow-up durations. Follow-up started at initiation of AED withdrawal. If a study provided information on e.g. one-year and five-year seizure outcome, it was only included in these two meta-analyses.

\begin{tabular}{|c|c|c|c|c|}
\hline Population & $\begin{array}{l}\text { Follow-up after } \\
\text { initiation AED } \\
\text { withdrawal }\end{array}$ & $\begin{array}{l}\mathrm{N} \text { studies } \\
\text { (n patients) }\end{array}$ & $\begin{array}{l}\text { Recurrence risk } \\
(95 \% \mathrm{Cl})\end{array}$ & $\begin{array}{l}\text { Heterogeneity } \mathrm{I}^{2} \\
(95 \% \mathrm{Cl})\end{array}$ \\
\hline \multirow[t]{4}{*}{$\begin{array}{l}\text { Medical cohorts } \\
\text { (in total } 45 \\
\text { studies, } 7082 \\
\text { patients) }\end{array}$} & 1 year & $36(5215)$ & $22.1 \%(18.7-26.0)$ & $88.8(82.3-93.5)$ \\
\hline & 2 years & 35 (5283) & $27.5 \%(23.7-31.6)$ & $89.0(82.5-93.5)$ \\
\hline & 3 or 4 years & $26(3697)$ & $33.7 \%(28.1-39.8)$ & $91.5(85.8-95.8)$ \\
\hline & 5 or more years & $19(3653)$ & $27.2 \%(22.8-32.2)$ & $87.3(77.5-94.5)$ \\
\hline \multirow[t]{4}{*}{$\begin{array}{l}\text { Surgical cohorts } \\
\text { (in total } 6 \text { studies, } \\
1172 \text { patients) }\end{array}$} & 1 year & $5(1115)$ & $13.7 \%(4.1-37.0)$ & 94.1 (83.0-99.2) \\
\hline & 2 years & $5(1115)$ & $21.0 \%(7.9-45.3)$ & $94.4(84.2-99.3)$ \\
\hline & 3 or 4 years & $5(1025)$ & $24.1 \%(12.5-41.6)$ & $88.4(67.5-98.4)$ \\
\hline & 5 or more years & $2(913)$ & $28.5 \%(0.0-100.0)$ & $88.4(0.7-98.4)$ \\
\hline
\end{tabular}




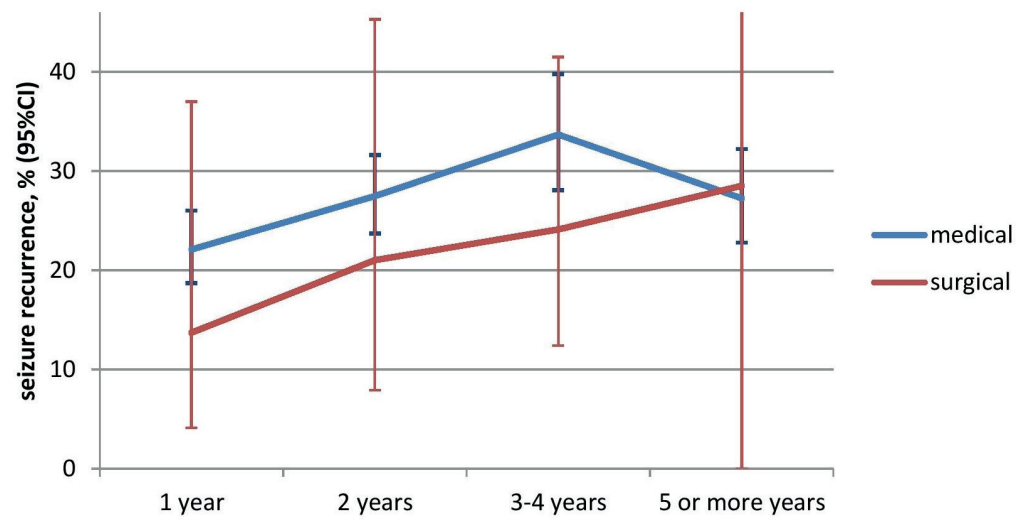

Figure 2 | Cumulative recurrence risk after AED withdrawal in medical and surgical cohorts. Error bars indicate $95 \%$ confidence interval. See table 1 for exact recurrence rates with $95 \% \mathrm{Cl}$.

The cumulative recurrence rate in surgical cohorts increased from 14\% (4\%-37\%) at one year after initiation of AED withdrawal, to $21 \%$ (8\%-45\%) at two years, $24 \%(13 \%-42 \%)$ at three to four years, and $29 \%$ (0\%-100\%) at five or more years. Six studies (1,172 patients) reported followup starting at the initiation of AED withdrawal and 11 studies (1,303 patients) started followup at surgery (one article reported both follow-up after surgery and after AED withdrawal). Meta-analysis of recurrence rates after AED withdrawal in these latter 11 articles is presented in Supplementary Table 2.

Heterogeneity between studies was high, with 12 of $88 \%$ to $94 \%$.

At all time points, there was no significant difference between the surgical and medical cohorts, but average recurrence rates were lower in surgical cohorts. Within the medical cohorts, $66 \%$ of recurrences occurred in the first year of follow-up. For surgical cohorts, this was $48 \%$.

\section{Study characteristics}

For the systematic review of predictors, study characteristics, seizure recurrences, and number of prognostic factors studied are listed in tables 2 and 3. In almost all cohorts of medically treated patients, a minimum of two years of seizure freedom was required before considering AED withdrawal, whereas this ranged from no minimum to a minimum of one year in the surgical articles. Clinical characteristics of included patients varied largely between cohorts, as evident from the different inclusion and exclusion criteria. Follow-up duration ranged from 22 to 216 months (median 48) after start of AED withdrawal in medically treated patients, and between 12 and 73 (median 61) after start of withdrawal in surgically treated patients and between 24 and 96 (median 58) months since surgery. 


\section{Quality assessment}

The articles that presented prognostic factors were assessed for potential bias. The quality was low to moderate in the majority of articles, as summarized in Supplementary Table 3. In most studies, the potential for bias in confounding measurement and account, and in analysis and reporting, was scored as moderate to high. In almost all articles, exploratory analyses were performed, in which the selection of variables into the multivariable model depended on the dataset and not on previous studies of prognostic factors. Because many of the potential predictors (or confounders) were not systematically included in each multivariable analysis, this increases potential for bias in confounding measurement and account (Hayden, Coté, and Bombardier 2006). The potential for bias in analysis and report is largely explained by the low number of included patients in most studies, compared to the large number of factors studied, resulting in low statistical power which leads to underestimation or false representation of predictive factors (Harrell Jr, Lee, and Mark 1996). Another reason for this large bias is that many articles did not fully report the results. In general, most articles had one or more flaws that increased the risk for bias, decreasing the scientific reliability of each of the individual studies.

\section{Prognostic factors}

Tables 4 and 5 list all variables that have been studied as possible risk factors for recurrence. The second column summarizes the number of studies in which a significant predictive value for a certain factor was identified compared to the number of studies that investigated the factor of interest. For example, 21 studies investigated the predictive value of female sex, and four concluded that this was a significant independent predictor for seizure recurrence.

In medically treated patients, 25 variables have been reported as significant independent predictors by at least one study, and 12 variables in surgical cohorts. 







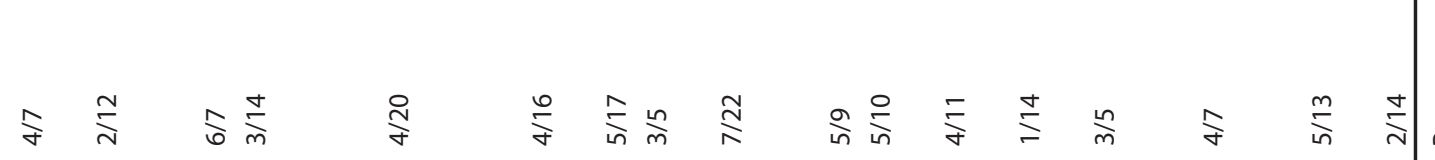

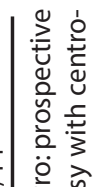

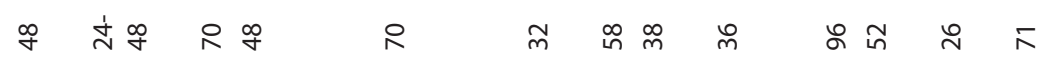

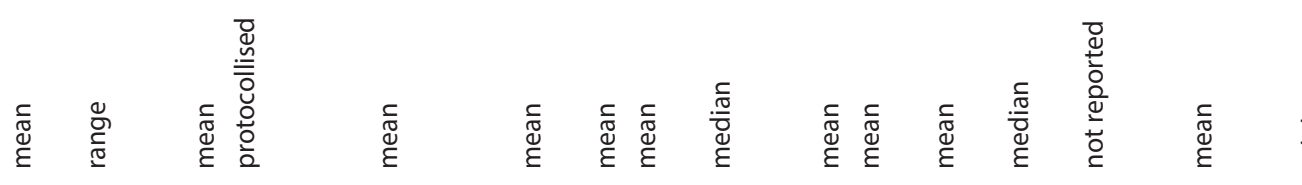

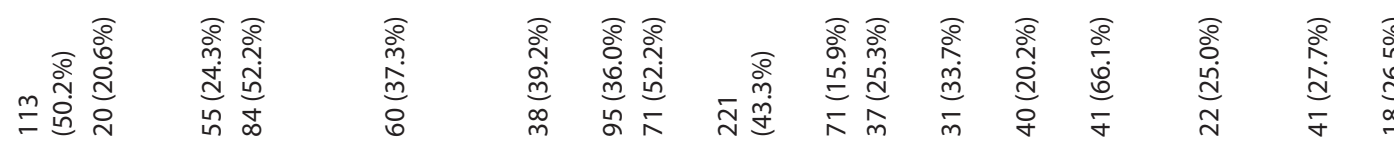
亗
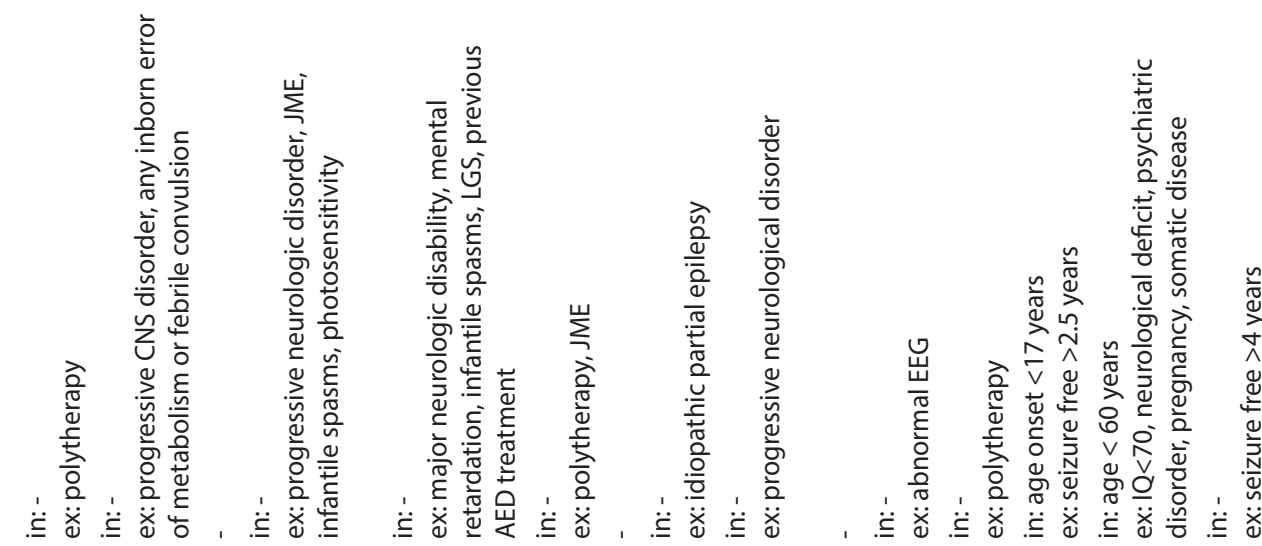
$\approx \stackrel{\circ}{\sim}$

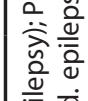




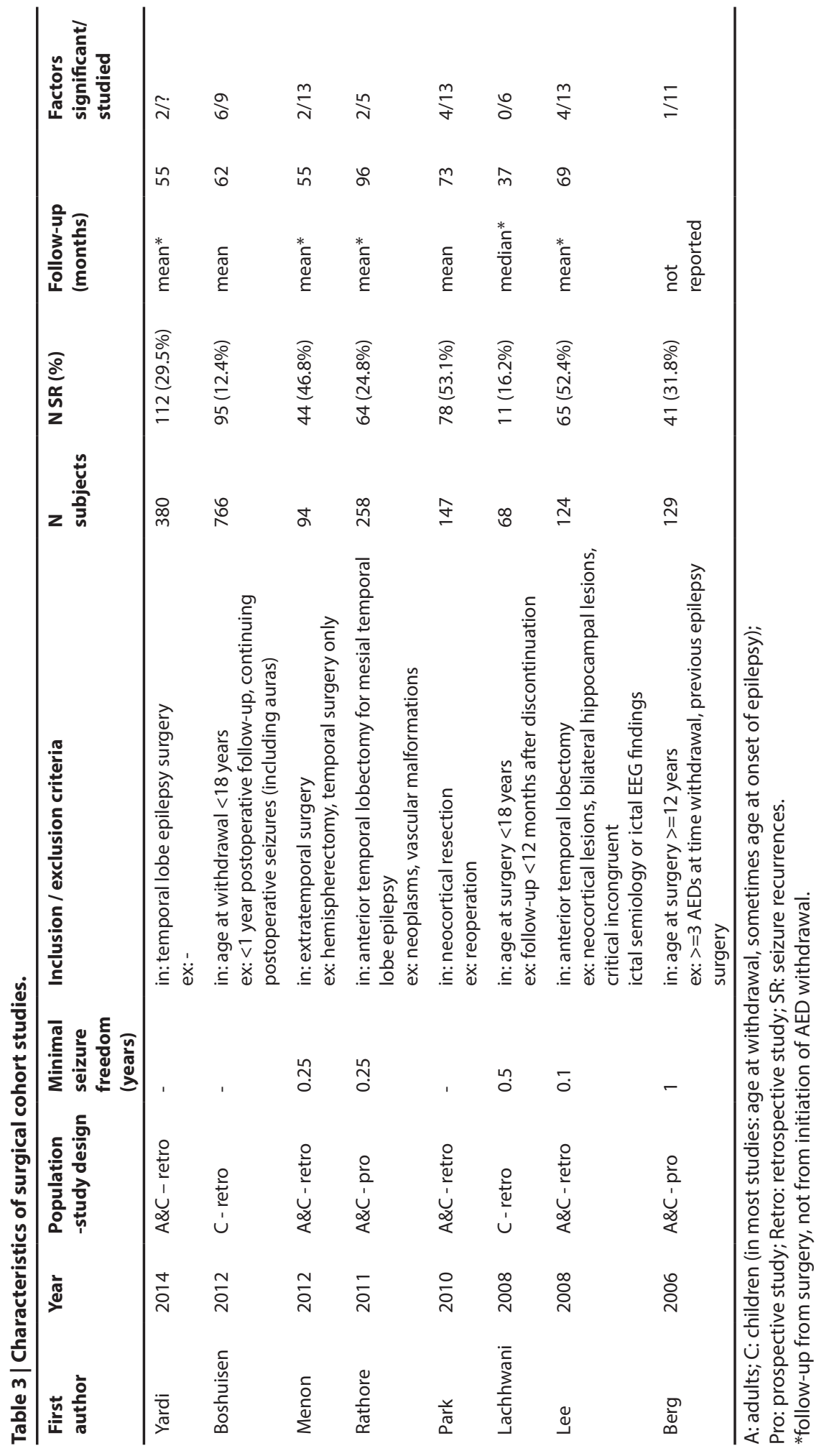


Table 4 | Risk factors for relapse in medically treated patients, showing significant findings based on multivariable models. Non-significant predictors that were only investigated by a single study were: age at initiation of treatment, time from first to second seizure, history of brain trauma, changes in seizure pattern over time, seizures only on awakening, seizures only while asleep, epilepsy severity, history of febrile seizures in patients with previously afebrile seizures, compliance, and previously attempted AED withdrawal. Correlations with different ethnic groups were studied in two articles and not found to be significant.

\begin{tabular}{lll}
\hline Predictor & $\begin{array}{l}\text { N significant/ } \\
\text { N studied }\end{array}$ & $\begin{array}{l}\text { References significant } \\
\text { findings }\end{array}$ \\
\hline $\begin{array}{l}\text { Patient } \\
\text { female sex }\end{array}$ & $4 / 21$ & $1-4$ \\
\hline Age and Time & & $2,5-11$ \\
$\quad$ age at onset & $8 / 22$ & $4,12-14$ \\
age at withdrawal & $4 / 13$ & 15 \\
age at last seizure & $1 / 2$ & $6,16,17$ \\
time from first seizure to start treatment & $0 / 6$ & 18 \\
time to seizure control (start treatment to last seizure) & $3 / 12$ & 11,19 \\
time from first seizure to last seizure (duration active disease) & $1 / 8$ & $13,14,18$ \\
time from start treatment to AED reduction (duration therapy) & $2 / 6$ & $3 / 10$ \\
time from last seizure to start AED reduction (duration seizure freedom) & \\
\hline
\end{tabular}

\section{Diagnostics}

neuroimaging (CT/MRI)

EEG abnormality before withdrawal

EEG abnormality or aggravation during/after withdrawal

\section{$0 / 8$}

$8 / 21$

$7 / 9$

9-11, 13, 19-21

$3,4,9,14,21-23$

\section{Aetiology}

remote symptomatic aetiology*

$\begin{array}{ll}3 / 13 & 1,8,24 \\ 6 / 13 & 1,2,12,17,21,25 \\ 4 / 12 & 10,12,17,21 \\ 0 / 3 & \end{array}$

\section{Seizure Characteristics}

seizure type ${ }^{* *}$

$9 / 24$

$2,8,9,11-13,17,19,21$

multiple types of seizures

$3 / 7$

$17,23,24$

seizure frequency/ amount of seizures before treatment

$2 / 16$

20,26

\section{History}

history of febrile seizures

$3 / 13$

$10,23,24$

history of neonatal seizures

$1 / 3$

24

history of status epilepticus

$0 / 7$

psychiatric abnormality

$1 / 3$

18

family history of epilepsy 
Table 4 continued

\begin{tabular}{lll}
\hline Predictor & $\begin{array}{l}\text { N significant/ } \\
\text { N studied }\end{array}$ & $\begin{array}{l}\text { References significant } \\
\text { findings }\end{array}$ \\
\hline Antiepileptic Drugs & & \\
$\quad$ number of AEDs & $4 / 13$ & $1,13,15,27$ \\
type of AED & $2 / 6$ & 12,19 \\
serum level of AED & $1 / 5$ & 15 \\
seizures after start AED treatment & $1 / 6$ & 13 \\
taper duration & $1 / 5$ & 5 \\
$\quad$ number of medication changes before remission & $0 / 3$ & \\
\hline
\end{tabular}

*unprovoked seizures related to static encephalopathy resulting from previous insult to the CNS (e.g. infection, CNS trauma, cerebrovascular disease) (ILAE, 1993)

${ }^{* * *}$ various seizure types have been reported as significant predictors, including partial seizures, absence seizures, rolandic seizures, "jacksonian seizures" andmyoclonic seizures.

(1) Arts et al., 1988; (2) Dooley et al., 1996; (3) Olmez et al., 2009; (4) Pavlovic et al., 2012; (5) Altunbasak et al., 1999;

(6) Ohta et al., 2004; (7) Bouma et al., 1987; (8) Peters et al., 1998; (9) Shinnar et al., 1985; (10) Shinnar et al., 1994; (11) Braathen and Melander, 1997; (12) Caviedes and Herranz, 1998; (13) MRC et al., 1993; (14) Galimberti et al., 1993; (15) Overweg et al., 1987; (16) Li et al., 2014; (17) Holowach-Thurston et al., 1982; (18) Specchio et al., 2002; (19) Callaghan et al., 1988; (20) Emerson et al., 1981; (21) Matricardi et al., 1989; (22) Su et al., 2013; (23) Verrotti et al., 2012; (24) RamosLizana et al., 2010; (25) Lossius et al., 2008; (26) Cardoso et al., 2003; (27) Vurucu et al., 2010. 
Table 5 | Risk factors for relapse in surgically treated patients, showing significant findings based on multivariable models. Non-significant predictors that were investigated only in a single study were: sex, age at surgical evaluation, time to complete discontinuation, poorly versus well-defined focal lesions, post resection ECoG spikes, SPECT preoperative ictal contralateral localization, PET preoperative contralateral localization, preoperative intracranial monitoring during evaluation, contralateral seizure semiology, persistent auras, temporal localization, lobar localization, type of surgery (lesionectomy versus bi/multilobar), MRI diagnosis of hippocampal sclerosis, tumour, and focal radiation.

\begin{tabular}{lll}
\hline Predictor & $\begin{array}{l}\mathbf{N} \text { significant/ N } \\
\text { studied }\end{array}$ & $\begin{array}{l}\text { References } \\
\text { significant findings }\end{array}$ \\
\hline Age and Time & & \\
$\quad$ age at onset epilepsy & $0 / 2$ & 1 \\
age at start withdrawal & $1 / 1$ & \\
age at surgery & $0 / 3$ & $1-3$ \\
$\quad$ time from surgery to reduction & $3 / 3$ & 3,4 \\
$\quad$ duration of epilepsy before surgery & $2 / 4$ & \\
\hline
\end{tabular}

\section{Diagnostics}

$\begin{array}{lll}\text { absence of focal MRI abnormalities } & 1 / 1 & 3 \\ \text { multifocal MRI lesions } & 1 / 1 & 2 \\ \text { EEG: preoperative contralateral interictal spikes } & 0 / 3 & \end{array}$

EEG: postoperative interictal epileptiform discharges prior to withdrawal

$4 / 5 \quad 2,4,5,6$

\section{Seizures}

$\begin{array}{lll}\text { generalized tonic-clonic seizures } & 0 / 2 & \\ \text { seizure frequency/ amount of seizures before treatment } & 1 / 4 & 6 \\ \text { history of febrile seizures } & 0 / 2 & 3,7 \\ \text { seizures between surgery and withdrawal } & 2 / 4 & \\ \text { acute postoperative seizures* } & 0 / 3 & \end{array}$

\section{Surgery Location}

lobar surgery

$0 / 2$

left-sided surgery

$0 / 2$

hemispherectomy

$1 / 1$

2

\section{Aetiology}

aetiology**

$1 / 5$

5

Therapy

$\begin{array}{lcc}\text { incomplete resection } & 1 / 2 & 2 \\ \text { number of AEDs } & 0 / 3 & \\ \text { multiple resections } & 1 / 1 & 2\end{array}$

*seizures within one week or two weeks after surgery, or seizures before hospital discharge

** significant for definite hippocampal sclerosis in a series of mesial temporal lobectomies

(1) Lee et al., 2008; (2) Boshuisen et al., 2012; (3) Park et al., 2010; (4) Menon et al., 2012; (5) Rathore et al., 2011;

(6) Yardi et al., 2014; (7) Berg et al., 2006. 


\section{Prediction models}

In addition to identification of predictors, some publications have created a clinical prediction model (Overweg et al. 1987; Medical Research Council 1993; Dooley et al. 1996; Braathen and Melander 1997; Geerts et al. 2005). These five models are compared in table 6, two of which reported c-statistics (0.73 and 0.78) (Medical Research Council 1993; Geerts et al. 2005). The populations vary (see also table 2), largely with respect to the inclusion of children or adults, the inclusion of subjects with mental or neurological impairment, and the required duration of seizure freedom. Therefore, most models are only applicable in highly specific populations; a general population with children and adults was used only in the model from the Medical Research Council (Medical Research Council 1993), although this model was built both on patients who withdrew AEDs and on those who continued AED treatment. There was no single predictive variable that was included in all models.

Table 6 | Existing prediction models for seizure recurrence after AED withdrawal.

\begin{tabular}{|c|c|c|}
\hline Publication & Population & Included variables \\
\hline Overweg (1987) & $\begin{array}{l}\text { age between } 18-60 \text { years }(n=62) \text {, } \\
\text { without mental or neurological } \\
\text { disability, seizure free }>3 \text { years }\end{array}$ & $\begin{array}{l}\text { (1) number of AEDs, (2) serum level of } \\
\text { AEDs, (3) age at last seizure, (4) dura- } \\
\text { tion of seizure free period }\end{array}$ \\
\hline $\begin{array}{l}\text { Medical Research } \\
\text { Council (1993) }\end{array}$ & $\begin{array}{l}\text { adults and children }(n=1003) \text {, } \\
\text { seizure free }>2 \text { years. Half of par- } \\
\text { ticipants randomized to maintain } \\
\text { AED treatment }\end{array}$ & $\begin{array}{l}\text { (1) age at withdrawal, (2) polytherapy, } \\
\text { (3) seizures after start of AED therapy, } \\
\text { (4) history of tonic-clonic seizures, } \\
\text { (5) history of myoclonic seizures, }(6) \\
\text { abnormal EEG in previous year, (7) no } \\
\text { EEG available, (8) duration of seizure } \\
\text { free period }\end{array}$ \\
\hline Dooley (1996) & $\begin{array}{l}\text { children }(n=97) \text {, seizure free }>1 \\
\text { year }\end{array}$ & $\begin{array}{l}\text { (1) female sex, (2) age at seizure onset, } \\
\text { (3) seizure type (generalized versus } \\
\text { partial), (4) neurologic abnormalities }\end{array}$ \\
\hline Braathen (1997) & $\begin{array}{l}\text { children without mental or neu- } \\
\text { rologic disability }(n=161) \text {, seizure } \\
\text { free }>1 \text { year }\end{array}$ & $\begin{array}{l}\text { (1) seizure type and epilepsy type } \\
\text { (complex partial, simple partial, ab- } \\
\text { sence epilepsy, generalized tonic clon- } \\
\text { ic seizures, rolandic seizures, benign } \\
\text { epilepsy with centrotemporal spikes), } \\
\text { (2) age at onset, (3) type of EEG abnor- } \\
\text { mality }\end{array}$ \\
\hline $\begin{array}{l}\text { Geerts }(2005, \\
\text { cohort of Peters } \\
1998)\end{array}$ & $\begin{array}{l}\text { children }(n=161) \text {, seizure free }>6 \\
\text { months }\end{array}$ & $\begin{array}{l}\text { (1) age at onset epilepsy. (2) absence } \\
\text { aetiology? If not: } \\
\text { (3) Idiopathic aetiology? (4) Abnormal } \\
\text { EEG? (5) Post-ictal signs? }\end{array}$ \\
\hline
\end{tabular}




\section{Discussion}

In this meta-analysis, the maximum cumulative recurrence risk at five or more years following the start of reduction was $34 \%$ in medically treated patients, and $29 \%$ in surgical cohorts. It can be concluded from the quality appraisal of studies that there is a moderate to high chance of bias (and therefore non-representative results) in most of the articles that reported on predictive factors of relapse. Concerning the existing prediction models on recurrence risk after AED withdrawal, we observed that no single predictor variable was included in each of the five models.

\section{Recurrence rates}

The presented recurrence rates for medically treated patients roughly resemble the outcome of a 20-year-old meta-analysis (Berg and Shinnar 1994) in which one-year and two-year recurrence rates of $25 \%$ (95\% Cl: $21 \%-30 \%)$ and $29 \%$ (24\%-34\%) were reported, respectively, which is only a few per cent higher than the results presented here. The current analysis adds longer-term seizure outcomes at three to four, and five or more years, revealing that seizures can recur after an interval longer than two years following the start of AED withdrawal, although this only happens in $6 \%$ of patients. Because our meta-analysis includes all available studies on recurrence risk after AED withdrawal until 2014, the average recurrence risks of $22 \%$ at one year, $28 \%$ at two years, and $34 \%$ at three or four years are the most accurate estimate currently available.

Seizure outcomes after AED withdrawal in surgical cohorts have recently been reviewed by Ladino et al. (Ladino, Hernández-Ronquillo, and Téllez-Zenteno 2014). Despite the comprehensive overview given, results are difficult to interpret, most importantly because the included articles had such a large range of follow-up durations, and only the recurrence rate at latest follow-up was used in their analysis. Cumulative relapse rates at specific time points were not provided. We therefore chose to determine the recurrence risk at different follow-up durations. We also used different inclusion criteria than those of Ladino et al. (Ladino, Hernández-Ronquillo, and Téllez-Zenteno 2014), excluding more articles from analysis, such as those describing only cohorts of patients who primarily underwent tumour resections. Bearing these differences in mind, we present recurrence rates of $14 \%$ at one year, $21 \%$ at two years, $24 \%$ at three or four years, and $29 \%$ at five or more years of follow-up after initiation of AED withdrawal.

\section{Predicting seizure recurrence}

In this systematic review, we listed the available evidence for different predictors of seizure recurrence after AED withdrawal. Although for some factors it seems likely that they predict relapse, for every variable there are also studies that found the opposite, and for some variables only few studies have reported significantly predictive values. Because most of the 
original studies reviewed here have several limitations, drawing definite conclusions from the presented data is not possible, and this may be the most important inference from this review.

An important issue, regarding the long lists of possible predictors, is how to decide which ones are relevant for clinical practice. An attempt to identify the most important predictors from this list is ill advised, because every method of doing so will have its limitations. As an example, it is tempting to presume that those factors that were most often reported as being significantly related to relapse are the strongest predictors. However, because of the limitations within all separate studies discussed below, conclusions will be weak at the least. Also, factors that have not been studied by many different groups would not be identified through this method, possibly unjustly so. In 1994, Berg and Shinnar performed a meta-analysis in which they studied only three predictors of seizure outcome after AED withdrawal and concluded that age at onset of epilepsy, symptomatic aetiology, and an abnormal EEG result, before AED withdrawal, significantly predicted seizure recurrence (Berg and Shinnar 1994). However, this was a meta-analysis based on univariable data, and therefore possible multicollinearity between these cannot be formally ruled out.

An obvious solution to this problem is a multivariable meta-analysis of all possible predictors. We have tried to meta-analyse the existing literature of predictors, but the large differences in presented outcome measures between studies made this impossible, given the mix of risk ratios, odds ratios and hazard ratios. A superior method of studying predictors is a meta-analysis of individual participant data (IPD), in which groups collaborate to create an aggregated dataset with predictor data at individual subject level which can be analysed multivariably (Riley, Lambert, and Abo-Zaid 2010). Only in this way, it becomes possible to study a large number of variables in a large dataset. This allows identification of predictors with accuracy which is not possible with other methods of meta-analysis, because the analysis can impute missing values on an individual patient level, detailed follow-up data is available, and (maybe most importantly) the analysis can be carried out in a standardized way across all included studies, while correcting for baseline characteristics (Riley et al., 2010). We have recently initiated a collaborative effort to perform such an IPD meta-analysis of all possible predictors of seizure relapse following AED withdrawal in both medically and surgically treated patients, as identified through this review.

\section{Limitations}

The recurrence risks calculated in our meta-analysis show estimates based on a relatively large number of articles and patients. Although many articles were included, not all presented useful information for risk estimation at each individual time point. In particular, in the surgical cohorts, only six of 16 articles presented follow-up starting from initiation of AED withdrawal, rendering ten articles inappropriate for primary analysis. The data derived from the analysis of 
the latter are not useful in daily practice, because the interval between surgery and withdrawal is different between individual patients and between centres.

In the medically treated patients, the cumulative recurrence risk after five or more years, calculated from all studies that included outcome at this follow-up duration, was lower than that after three to four years. The explanation for this discrepancy is that most articles presenting long-term follow-up did not include information also on the one- to four-year follow-up time point, and had a lower overall recurrence rate. For this reason, the presented data should not be taken as exact hazard functions; this would only be possible when all studies provided information on recurrences at all time points, or even better, when individual patient data were aggregated into a large IPD meta-analysis. In an additional analysis of studies that contained data from both follow-up time points, we showed that relapse rate at five or more years after start of AED withdrawal was virtually identical to that at three to four years.

The current presentation of evidence concerning predictors of relapse has several limitations. First, several factors have been measured or defined variably across the different studies. As an example, age at onset of epilepsy was measured both as a continuous variable and dichotomized, and the cut-off age ranged between 2 and 12 years. Second, the inconsistency between study results may be caused by low sample sizes, as mentioned before. Third, there might be true differences in predictors between different populations. Fourth, the methodology of statistical analysis varied greatly between studies, and different variables were included in the multivariable models leading to heterogeneous results; effects of multicollinearity may be present in one but absent in another model because of inclusion or exclusion of certain variables. As argued by John loannidis (loannidis 2005), several factors increase the possibility of "false positive findings"; amongst others: small sample sizes, high possibility for bias, hypothesis-generating studies (compared to confirmatory designs), and greater flexibility in design, analysis and reporting. All these factors are present in the different studies included here, increasing the chance that some of the findings are in reality not true. The way forward is to perform larger, bias-free studies or meta-analyses with collaboration, more appropriate statistics, and standardization (loannidis 2005, 2014).

Concerning the existing prediction models presented in table 6 , several limitations also exist. To start with, the populations on which the models have been based on raise questions of generalizability. Three articles have based their model on a paediatric population, one on an adult population, and one on a population with children and adults. It has been shown before (Berg and Shinnar 1994) that adolescent age at onset of epilepsy has a higher risk of recurrence than both childhood and adult-onset epilepsy. By excluding an age group, this effect might be less prominent or even absent. Also, two articles excluded patients with low intelligence quotient or neurological deficits. These two factors, which can be seen as signs of symptomatic 
aetiology, seem to be predictors of worse outcome based on the results listed in table 4, and also on a previous meta-analysis (Berg and Shinnar 1994). By excluding these patients from analysis, the validity of the model is restricted. In addition, the required duration of seizure freedom before the start of AED reduction varied between six months and three years, whilst three reports suggested that this time is important in determining recurrence risk (table 4). The above-mentioned observations illustrate that a narrative systematic review is only the first step in determining which factors will ultimately aid decision-making in the clinic. Unfortunately, no conclusions can yet be drawn concerning each separate predictor. Future research in the form of larger and well-designed analyses or IPD meta-analysis is needed to be able to predict recurrence risk in individual patients.

Lastly, an important limitation to the current review is the absence of effect sizes. Even if the conclusion would be that a certain factor predicts seizure recurrence, this review does not give insight into the strength of that prediction. Future quantitative analyses should provide answers to the question of effect sizes.

\section{Conclusion}

Survival analyses revealed that cumulative risk of seizure recurrence after AED withdrawal is $29 \%$ in surgically treated and $34 \%$ in medically treated patients. Many factors have been identified as possible predictors of seizure recurrence after AED withdrawal, but the evidence is inconclusive. To determine which variables are truly and independently predictive, and to assess effect sizes, the findings from this systematic review should be incorporated into a large meta-analysis of individual patient data. 
Supplementary Table 1 | Subgroup analysis from studies that described recurrence risks in medically treated cohorts both at 3-4 years and at 5 or more years of follow-up.

\begin{tabular}{llll}
\hline Follow-up & N studies (n patients) & Recurrence risk $\mathbf{( 9 5 \% ~ C I )}$ & Heterogeneity $\left.\mathbf{I}^{\mathbf{2}} \mathbf{( 9 5 \%} \mathbf{C I}\right)$ \\
\hline $3-4$ years & $7(905)$ & $28.9 \%(22.5-36.4)$ & $72.0(33.3-93.7)$ \\
5 or more years & $7(905)$ & $29.6 \%(22.9-37.3)$ & $73.5(36.8-94.0)$ \\
\hline
\end{tabular}

Supplementary Table 2 | Meta-analysis of recurrence risk after AED withdrawal in surgical cohorts, in studies that reported follow-up from surgery instead of follow-up from initiation AED withdrawal.

\begin{tabular}{|c|c|c|c|c|}
\hline & $\begin{array}{l}\text { Follow-up after } \\
\text { surgery }\end{array}$ & $\begin{array}{l}\mathbf{N} \text { studies ( } \mathbf{n} \\
\text { patients) }\end{array}$ & $\begin{array}{l}\text { Recurrence risk (95\% } \\
\mathrm{Cl})\end{array}$ & $\begin{array}{l}\text { Heterogeneity } \mathrm{I}^{2} \\
(95 \% \mathrm{CI})\end{array}$ \\
\hline \multirow[t]{4}{*}{$\begin{array}{l}\text { Surgical cohorts } \\
\text { (in total } 11 \text { studies, } \\
1303 \text { patients) }\end{array}$} & 1 year & $1(84)$ & $9.5 \%(-)$ & - \\
\hline & 2 years & $3(217)$ & $19.6 \%(5.8-49.2)$ & $56.7(0.0-99.4)$ \\
\hline & 3 or 4 years & $4(598)$ & $27.4 \%(14.8-45.0)$ & $83.2(46.5-97.9)$ \\
\hline & 5 or more years & $5(640)$ & $33.9 \%(22.8-47.1)$ & $82.6(54.6-97.0)$ \\
\hline
\end{tabular}


Supplementary Table 3 | Quality appraisal of prognostic studies (Hayden et al., 2006)

\begin{tabular}{|c|c|c|c|c|c|c|}
\hline Publication & $\begin{array}{l}\text { Study } \\
\text { participation }\end{array}$ & $\begin{array}{l}\text { Study } \\
\text { attrition }\end{array}$ & $\begin{array}{l}\text { Prognostic } \\
\text { factor } \\
\text { measurement }\end{array}$ & $\begin{array}{l}\text { Outcome } \\
\text { measurement }\end{array}$ & $\begin{array}{l}\text { Confounding } \\
\text { measurement } \\
\text { and account }\end{array}$ & $\begin{array}{l}\text { Analysis } \\
\text { and } \\
\text { reporting }\end{array}$ \\
\hline
\end{tabular}

\section{Medical cohorts}

Li, 2014

Su, 2013

Pavlovic, 2012

Verrotti, 2012

Ramos-Lizana, 2010

Vurucu, 2010

Olmez, 2009

Lossius, 2008

Geerts 2005

Ohta, 2004

Cardoso, 2003

Specchio, 2002

Altunbasak, 1999

Caviedes, 1998

Braathen, 1997

Dooley, 1996

Shinnar, 1994

Galimberti, 1993

MRC, 1993

Matricardi, 1989

Arts, 1988

Callaghan, 1988

Bouma, 1987

Overweg, 1987

Shinnar, 1985

Holowach, 1982

Emerson, 1981

Surgical cohorts

Yardi, 2014

Boshuisen, 2012

Menon, 2012

Rathore, 2011

Park, 2010

Lee, 2008

Lachhwani, 2008

$+/-$

$+/-$ 







\section{Cumulative effects of antiepileptic drugs on intelligence in children with focal epilepsy}

Carmen H. Stevering Herm J. Lamberink Julie Woodfield Monique van Schooneveld Willem M. Otte Karin Geleijns

Kees P.J. Braun 


\begin{abstract}
Objective: Antiepileptic drugs may have long-term effects on the neurodevelopment of children. We aimed to investigate the association between cumulative antiepileptic drug load and intelligence quotient (IQ), in relation to brain volume and cortical thickness and volume.
\end{abstract}

Methods: Retrospective analysis of children with focal epilepsy who underwent neuropsychological assessment and an MRI between the ages of 5-12 years in a tertiary epilepsy center. Cumulative drug load was presented in drug-years. We studied the association between drug load and IQ with multivariable linear regression, corrected for the following epilepsyrelated confounders: age at first treatment, etiology, maximum seizure frequency, duration of active epilepsy, history of secondary generalized seizures, history of status epilepticus and number of antiepileptic drugs at time of neuropsychological assessment.

Results: We included 59 children, median drug load was 5.3 drug-years (interquartile range: 2.0 - 11.1), mean total IQ ( \pm standard deviation) 77.4 \pm 18.9 . A significant negative relation between drug load and total IQ was found with a decrease of 1.2 IQ-points per drug-year (95\% confidence interval: -2.1 to -0.3 ), after correcting for confounders. Drug load was not significantly associated with brain volume, or cortical thickness, nor were the latter with IQ.

Conclusions: Higher drug load is associated with lower total IQ after adjusting for epilepsyrelated confounders. No evidence for a volumetric cause underlying the drug related IQ decrease was found. Prospective, longitudinal confirmation of these findings is required. Lastly, it should be stressed that effective seizure prevention outweighs potential negative effects of antiepileptic drugs. 


\section{Introduction}

Epilepsy is one of the most common brain disorders in children, with a prevalence of approximately $1 \%$ of all children (Russ, Larson, and Halfon 2012). A major issue in children with epilepsy are cognitive problems. These may be caused by the underlying pathology (Åndell et al. 2015; Reilly et al. 2015) and epilepsy-related factors (Kim and Ko 2016; Braun 2017), but an additional reason for concern is the treatment. Primary treatment of epilepsy consists of antiepileptic drugs (AEDs), which can suppress seizures by various mechanisms of action. Many studies have demonstrated that children who are using these drugs experience cognitive side effects (Aldenkamp et al. 2016; Lagae 2017, 2006; Bath and Scharfman 2013; Loring and Meador 2004). These studies, however, did not look at any potential long-term effects. The only knowledge we have on potential harmful effects of AED use on neural development comes from two fields of research: rodent studies (Bath and Scharfman 2013) and studies on the children of women who were taking AEDs during pregnancy. Prenatal exposure to AEDs affects brain development and results in an increased risk of intellectual disability, language impairment, psychomotor decline and autism spectrum disorders (Bath and Scharfman 2013; Verrotti et al. 2014; Scolnik et al. 1994; Koch et al. 1999; Reinisch et al. 1995)

Neurodevelopment, however, does not stop after birth. Between the ages of one and six, critical processes take place such as maturation of cortical structures and the establishment of their connectivity (Deoni et al. 2015). Furthermore, dendrite growth, synaptogenesis and myelination, amongst others, continue until adulthood (Webb, Monk, and Nelson 2001). Only one study has shown potential long-term effects of AEDs on intelligence quotient (IQ), which was a trial using phenobarbital in children with febrile seizures (Farwell et al. 1990; Sulzbacher et al. 1999). However, this is not informative for current practice, as this drug is now rarely prescribed beyond the neonatal age. Because the mechanism of action of AEDs includes many pathways that are also important in these developmental processes, we hypothesized that the use of AEDs early in life may affect brain development and cognitive functioning.

To test this hypothesis, this study assessed the long-term effect of exposure to AEDs on IQ, as a broad measure of neurodevelopment. The secondary aim was to investigate whether cortical thickness and brain volume measures could explain the relation between AEDs and IQ.

\section{Methods}

Study population

In this retrospective cohort study data was obtained from children with epilepsy (Fisher et al. 2014) evaluated and followed at our outpatient child neurology clinic, our first seizure clinic, and those who were evaluated for epilepsy surgery, between 2005 and 2017. Children were included when they suffered from focal epilepsy according to the International League Against 
Epilepsy classification (Fisher et al. 2017). Additional inclusion criteria were the availability of a neuropsychological assessment (NPA) during or after drug treatment, and a magnetic resonance brain scan for measuring cortical thickness and volumes (T1-weighed 3D MRI at 1.5 or 3.0 Tesla). Both NPA and MRI had to be performed between the age of five and twelve years. Children with NPA or MRI below the age of five years were excluded because of the difficulty of reliable automatic segmentation of MRI due to poor grey-white differentiation and shape differences to adult templates in immature brains (Vân Phan et al. 2018). Age at first treatment could have been before the age of 5 years. The maximum age of twelve years was chosen to limit the age span and reduce study population heterogeneity. To study the effect of AEDs on the cortex, only children were included with focal epilepsy refined to a single hemisphere based on semiology, (inter)ictal EEG, and in case of structural etiologies the exclusion of contralateral MRI-lesions. Furthermore, patients were excluded when there was a risk of a diffusely affected brain, for instance patients with a history of generalized epilepsy, tuberous sclerosis complex, mitochondrial or other metabolic disease, or children who had suffered from epileptic encephalopathies at some point during the course of their disease (Box 1). Similarly, patients suffering from epilepsy requiring hemispherectomy were excluded. Patients with a history of oral steroid treatment or thiopenthal coma were excluded from analysis as these interventions may influence total brain volume. Lastly, patients were excluded when they underwent epilepsy surgery before NPA and acquisition of MRI, as epilepsy surgery itself has an impact on cognition (Braun 2017).

The Dutch Medical Research Involving Human Subjects Act did not apply, as confirmed by the Ethical Committee of the University Medical Center in Utrecht. Patients with an active treatment relation at this center were approached by the researcher to obtain informed consent. Patients who previously objected to being part of scientific research were excluded.

\section{BOX 1 | Definitions.}

Aetiology of epilepsy: genetic, structural, metabolic, immune, infectious, unknown; according to International League Against Epilepsy classification (Scheffer et al. 2017).

Duration of active epilepsy: calculated as the difference between the date of first seizure and the date of last seizure expressed in years. Date of NPA was used when completed prior to the last seizure, or when the date of last seizure was unavailable.

Maximum seizure frequency: defined as the highest seizure frequency as noted in the patient's history before NPA was performed, expressed in seizures per day.

History of secondary generalised seizures: scored positively if a secondary generalised seizure was reported.

History of status epilepticus: scored positively if any case of status epilepticus requiring hospitalisation was reported. Status epilepticus was classified according to the International League Against Epilepsy guidelines (Trinka et al. 2015). 


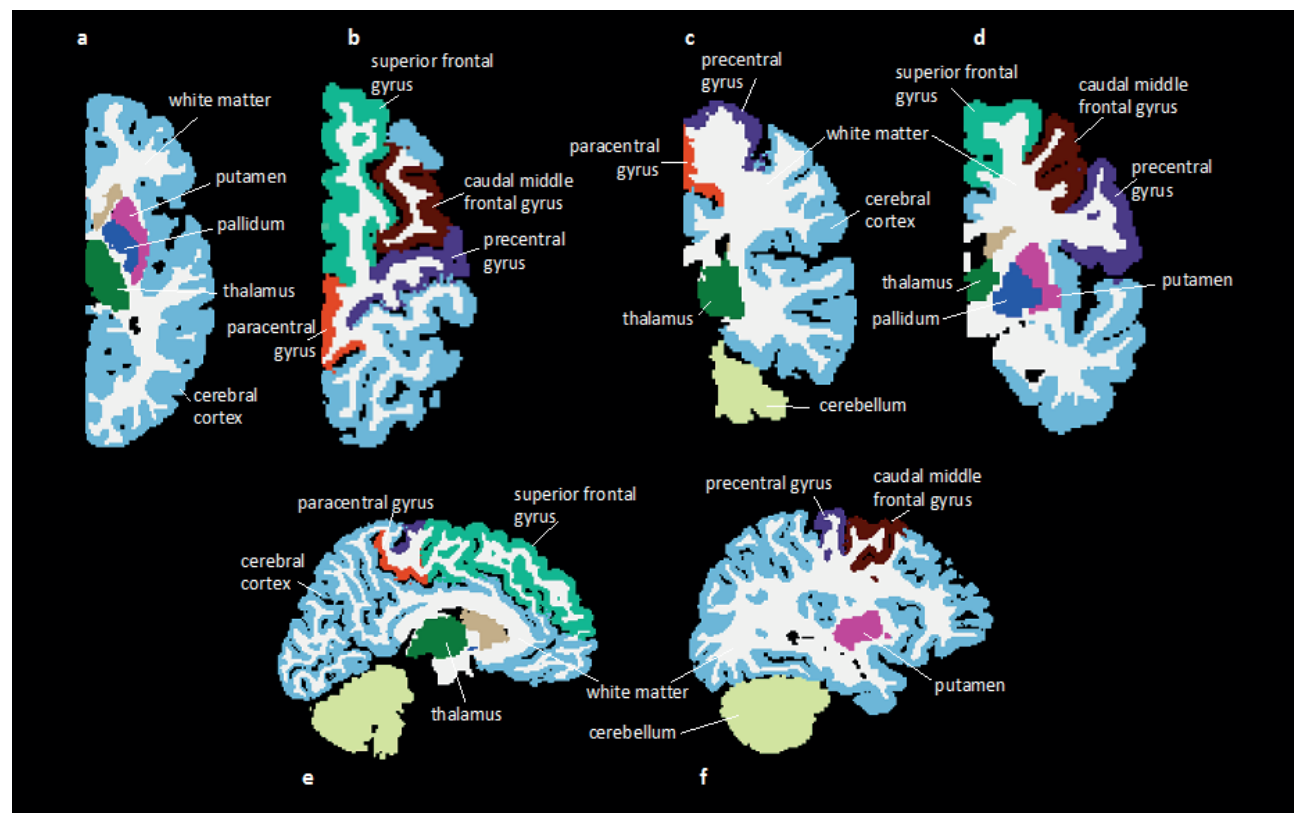

Figure 1 | Anatomical regions parcellated from T1-weighted MRI using Freesurfer. A representative left hemisphere is shown.

a) inferior axial slice, b) superior axial slice, c) posterior coronal slice, d) anterior coronal slice, e) medial sagittal slice, f) lateral sagittal slice. Regions shown: white matter (white), cerebral cortex (light blue), putamen (pink), pallidum (dark blue), thalamus (dark green), caudate (light brown), superior frontal gyrus (light green), caudal middle frontal gyrus (dark brown), precentral gyrus (purple), paracentral gyrus (red), cerebellum (yellow).

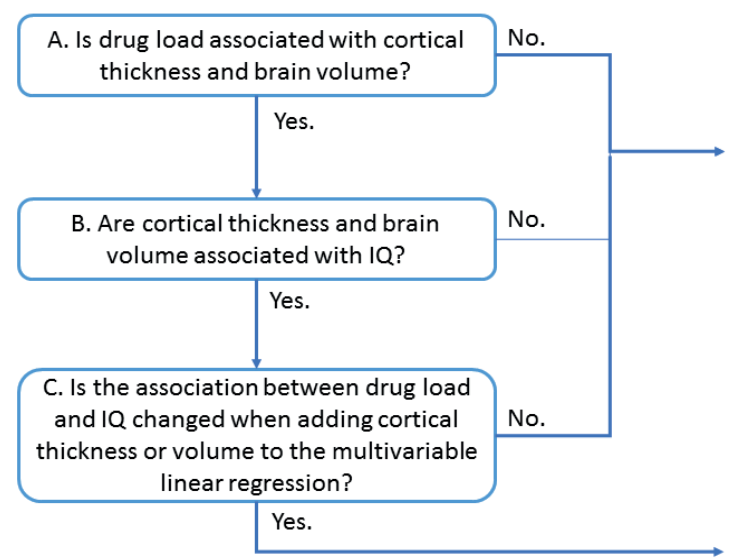

Cortical thickness and brain volume are not mediators of the association between drug load and IQ

Cortical thickness or brain volume explains (part of) the association between drug load and IQ

Figure 2 | Mediation analysis: is the association between drug load and IQ explained by an effect on brain development? 


\section{Drug load calculations}

Cumulative drug load was calculated by adding the years each AED was taken until the NPA. Since the effect of long-term exposure to AEDs was our primary interest, rescue medication for acute seizures was not considered in this calculation. Drug load is defined in units of drug-years, where one drug-year corresponds to one AED being taken for one year. As an example, when a child used both valproate and levetiracetam for one year during the timespan preceding NPA, drug load was 2 drug-years. Data was also collected regarding the number of drugs taken at time of NPA and MRI, as potential confounders.

\section{Brain structure measurements}

The MRI sequences were visually inspected for quality and scans with excessive artefact precluding automated processing were excluded. Scans were segmented and parcellated using Freesurfer Version 5.3.0 (Fischl et al. 2002). Cortical thickness (Fischl and Dale 2000) was calculated at each surface vertex as the distance from the grey white boundary to the cortical surface using the regions of the Desikan-Killiany atlas (Desikan et al. 2006). All segmentations and parcellations were manually checked for accuracy and manual adjustments to registration, parcellation, and segmentation were made where necessary. Only measurements from the hemisphere from which seizures did not arise were considered. The mean cortical thickness of the entire healthy hemisphere, contralateral to the seizure focus, as well as several specified cortical regions were analyzed. The precentral gyrus, caudal middle frontal gyrus, paracentral gyrus and superior frontal gyrus were specifically investigated because cortical thickness in these regions has been reported to be most affected in people with epilepsy (Whelan et al. 2018). Cerebral volume changes were studied investigating total hemisphere volume and the ratios between white matter volume / total hemisphere volume, and cerebellar volume / total hemisphere volume. Volumes of thalamus, pallidum and putamen were also analyzed, following the findings described by Whelan and colleagues (Whelan et al. 2018). Regions analyzed are displayed in Figure 1.

\section{Endpoints of interest}

The primary endpoint was IQ, which was assessed in children who underwent neuropsychological assessment for clinical reasons at the discretion of the treating physician. Whenever multiple NPAs were available, the latest was included. All NPAs were carried out by a clinical child neuropsychologist. Each NPA resulted in standardized scores for total intelligence quotient (TIQ), verbal intelligence quotient (VIQ) and performance intelligence quotient (PIQ).

\section{Data collection and analysis}

Data were obtained from patient records and consisted of general patient characteristics, epilepsy characteristics, results of structural MRI as concluded by an epilepsy-dedicated neuroradiologist, detailed information on drug therapy, and results from NPA. Cognitive 
outcome in epilepsy is influenced by multiple factors (Braun 2017; Kim and Ko 2016): etiology of epilepsy, maximum seizure frequency, duration of active epilepsy, age at onset of AED treatment, history of secondary generalized seizures, history of status epilepticus requiring hospitalization and the number of AEDs used during NPA. Since these factors may also influence the drug load, they were predefined as potential confounders. Definitions of the confounders are listed in Box 1.

For the primary endpoint, we tested the relation between drug load and IQ univariably using linear regression to get a crude estimate of the association. Next, we added all possible confounders to the regression model to obtain an adjusted regression coefficient. Children with IQ $<70$ are considered to have cognitive impairment; as a sensitivity analysis, all patients with an IQ $<70$ were excluded and the analysis was repeated. Secondly, to assess the hypothesis that the effect of drug load on IQ was mediated by changes in cortical structures or brain volume, a mediation analysis (Baron and Kenny 1986) was performed in three steps as visualized in Figure 2.

Statistical analysis was performed using SPSS Statistics (Version 25). For the primary analyses analysis, $\mathrm{p}<0.05$ was considered statistically significant. For the secondary analyses, a Bonferroni correction for multiple testing was applied resulting in an alpha level of $0.05 / 11=$ 0.0045 .

\section{Data availability statement}

Anonymized data will be shared by request from any qualified investigator.

\section{Results}

We screened 1658 patients for eligibility, resulting in 59 eligible patients (Supplementary Figure 1). Most frequent reasons for exclusion were no epilepsy ( $n=885)$, no MRI acquired in our own center $(n=305)$, no focal epilepsy $(n=148)$ and no available MRI and/or NPA between the age of 5-12 years $(n=129)$. Baseline characteristics of the study participants are presented in Table 1. Median drug load (interquartile range (IQR)) was 5.3 drug-years (2.0 - 11.1) and the median duration of active epilepsy was 4.1 years $(1.9-6.7)$. The timing of the MRI and NPA was closely related, with an median (IQR) duration of active epilepsy until NPA being 4.1 years (1.7 - 6.7), and until MRI 4.1 years (1.9 - 6.7). The most commonly used drugs were valproic acid (57 patients), clobazam (38 patients) and carbamazepine (37 patients) (Table e-1). Mean ( \pm SD) IQ scores were TIQ $77.4( \pm 18.9)$, VIQ $83.9( \pm 18.2)$, and PIQ $80.3( \pm 15.0)$.

An increase in drug load was associated with a decrease in TIQ. This association was significant in both the crude linear regression (regression coefficient (RC) -1.7 per drug-year; 95\% confidence interval $(95 \% \mathrm{Cl})-2.2$ to -1.1$)$ and the adjusted model $(\mathrm{RC}-1.2 ; 95 \% \mathrm{Cl}-2.1$ to -0.3$)$ (Table 2 and Table e-2). Analyses for both VIQ and PIQ showed that an increase in drug load was 
significantly associated with a decrease in VIQ or PIQ (Figure 3). This was significant in the crude model only (VIQ RC $-1.5 ; 95 \% \mathrm{Cl}-2.2$ to -0.8 ; PIQ RC $-1.2 ; 95 \% \mathrm{Cl}-1.9$ to -0.5 ). These associations lost significance after adjustment for potential epilepsy-related confounders (Table 2 and Table e-2). Sensitivity analyses for patients with $\mathrm{TIQ}^{3} 70$ revealed a significant association for $\mathrm{TIQ}$ and PIQ in multivariate regression analyses (TIQ RC $-1.9 ; 95 \% \mathrm{Cl}-3.2$ to $-0.5 ; \mathrm{PIQ} R C-2.6 ; 95 \% \mathrm{Cl}-4.0$ to -1.1), although not for VIQ (RC $-1.2 ; 95 \% \mathrm{Cl}-3.0$ to 0.6 ) (Table e-3). Table e-4 breaks down the effect of AED load into drug load during monotherapy and drug load during polytherapy. The adjusted effects are similar to the main effect described in Table 2 with the RC of -1.2.

Linear regression analyses between drug load and brain thickness and volume measures did not reveal a significant association in both crude and adjusted models with the Bonferroni corrected alpha-level of 0.0045 (Table 3). Additionally, no significant association was found between cortical thickness and volumes of each of the individual brain structures and TIQ, VIQ and PIQ scores (Table 4). The third step of the mediation analysis (Figure 2) was not executed because the first two steps did not show any significant association between drug load and brain structure nor between brain structure and IQ.

Interestingly, in the adjusted models thalamus volume tended to correlate with IQ, only becoming significant for performance IQ, after adjustment for other factors: RC 7.1 IQ points per $\mathrm{mm}^{3}$ volume increase $(95 \% \mathrm{Cl} 0.8$ to $13.4, \mathrm{p}=0.027$ ) (Table 4). However, this variable did not cross the Bonferroni-corrected alpha-level of 0.0045 and should therefore be considered non-significant.

Table 1 | Demographics.

\begin{tabular}{ll}
\hline Variable & Mean (SD)/median (IQR; Q1 - Q3)/n (\%) \\
\hline Gender, female & $31 / 59(52.5 \%)$ \\
Age at first seizure, years & $4.0(1.2-5.5)$ \\
Age at first AED treatment, years & $4.6(2.0-6.6)$ \\
Duration of active epilepsy*, at time of: & \\
MRI & $4.1(1.7-6.7)$ \\
NPA & $4.1(1.9-6.7)$ \\
Time between MRI and NPA§ & $-0.2(1.9)$ \\
Etiology of epilepsy & \\
Structural & $42(71.2 \%)$ \\
Genetic & $2(3.4 \%)$ \\
Metabolic & $1(1.7 \%)$ \\
Infectious & $1(1.7 \%)$ \\
Immune & $0(0.0 \%)$ \\
Unknown & $13(22.0 \%)$ \\
Seizure frequency & \\
Maximum seizure frequency MRI per month† & $91.3(2.0-243.3)$ \\
Maximum seizure frequency NPA per month† & $91.3(2.0-243.3)$ \\
Seizure frequency near MRI per month & $8.7(0.2-121.7)$ \\
Seizure frequency near NPA per month & $5.4(0.2-76.0)$ \\
& \\
\hline
\end{tabular}


Table 1 continued

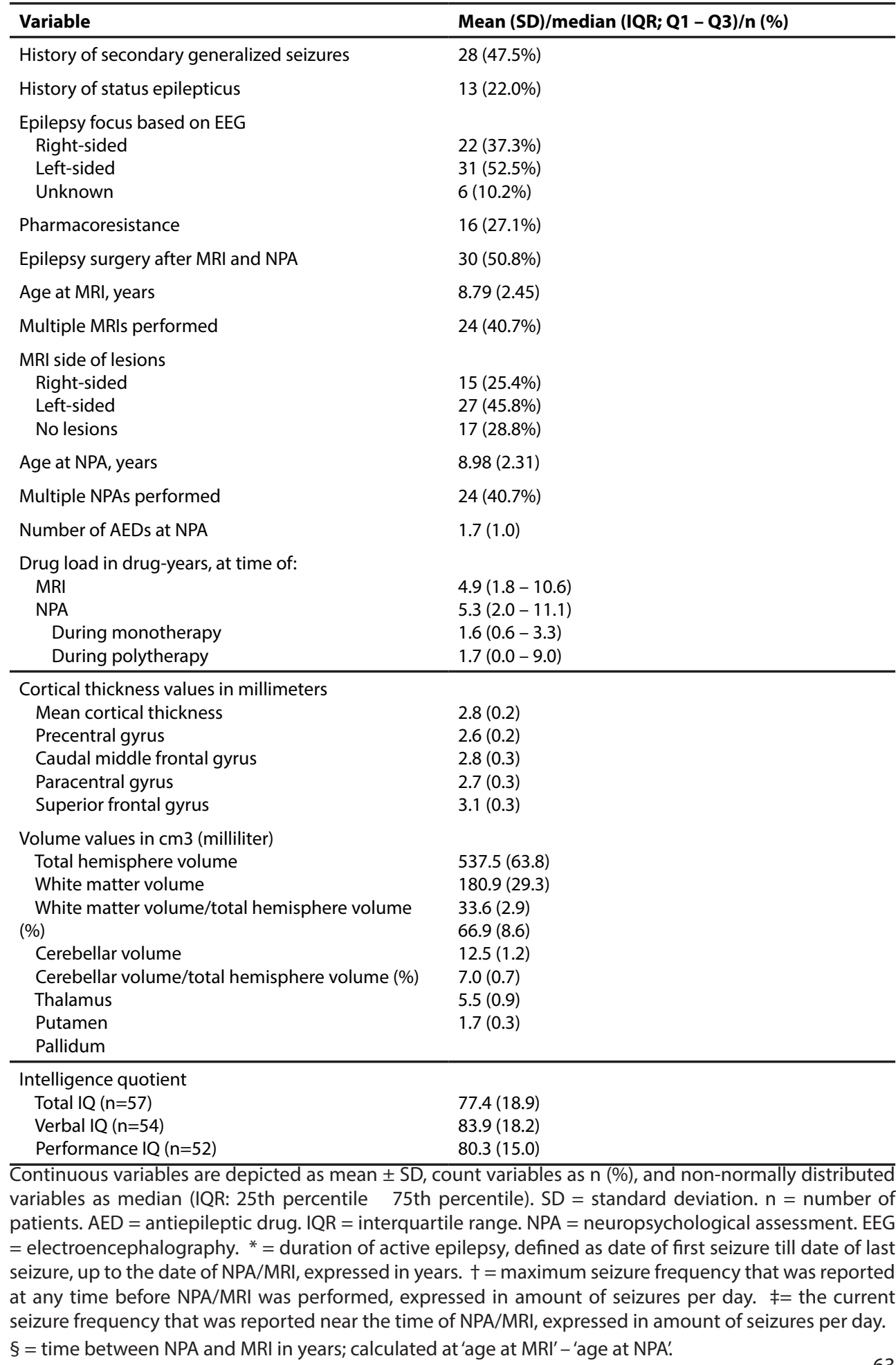




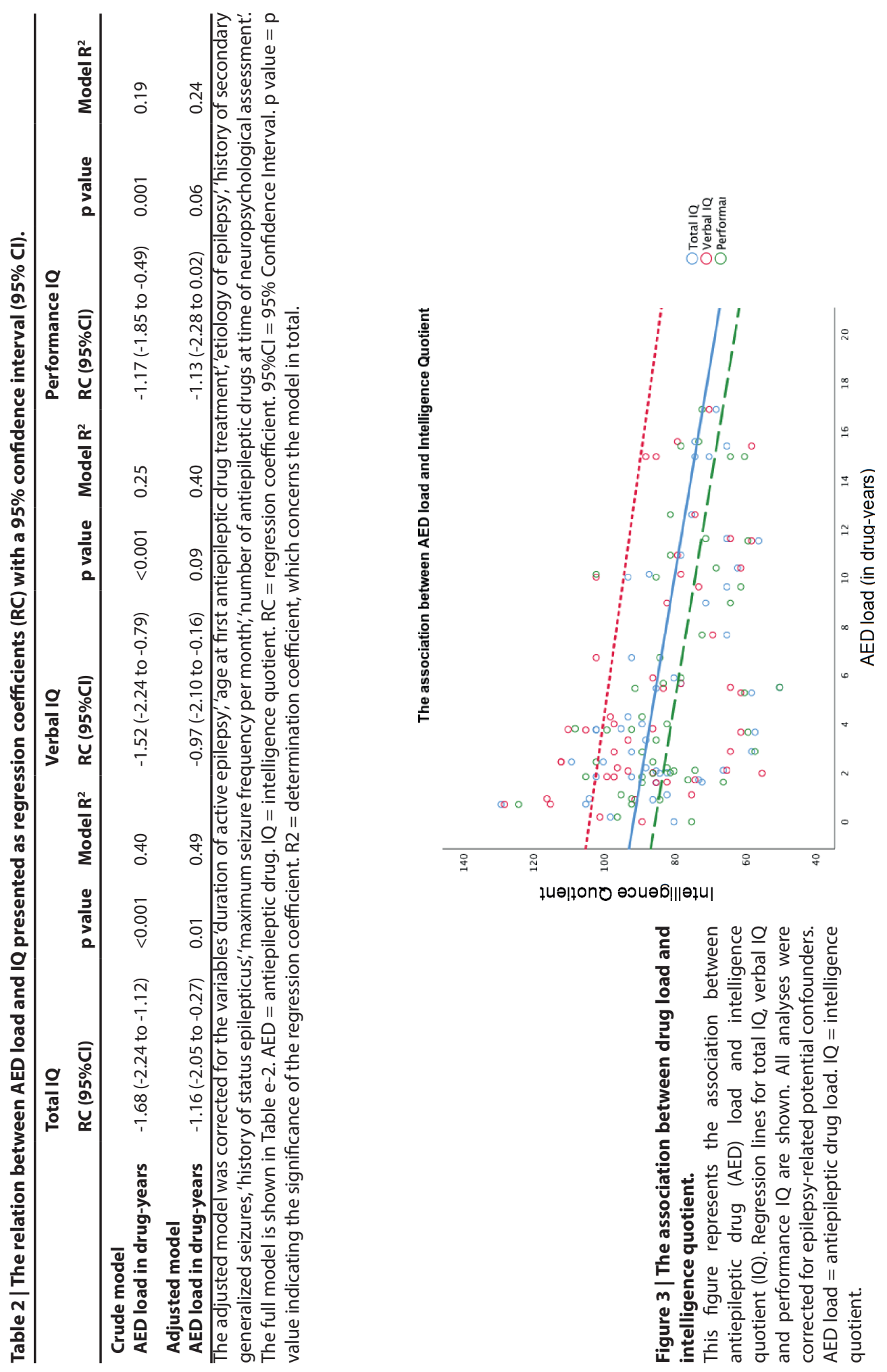














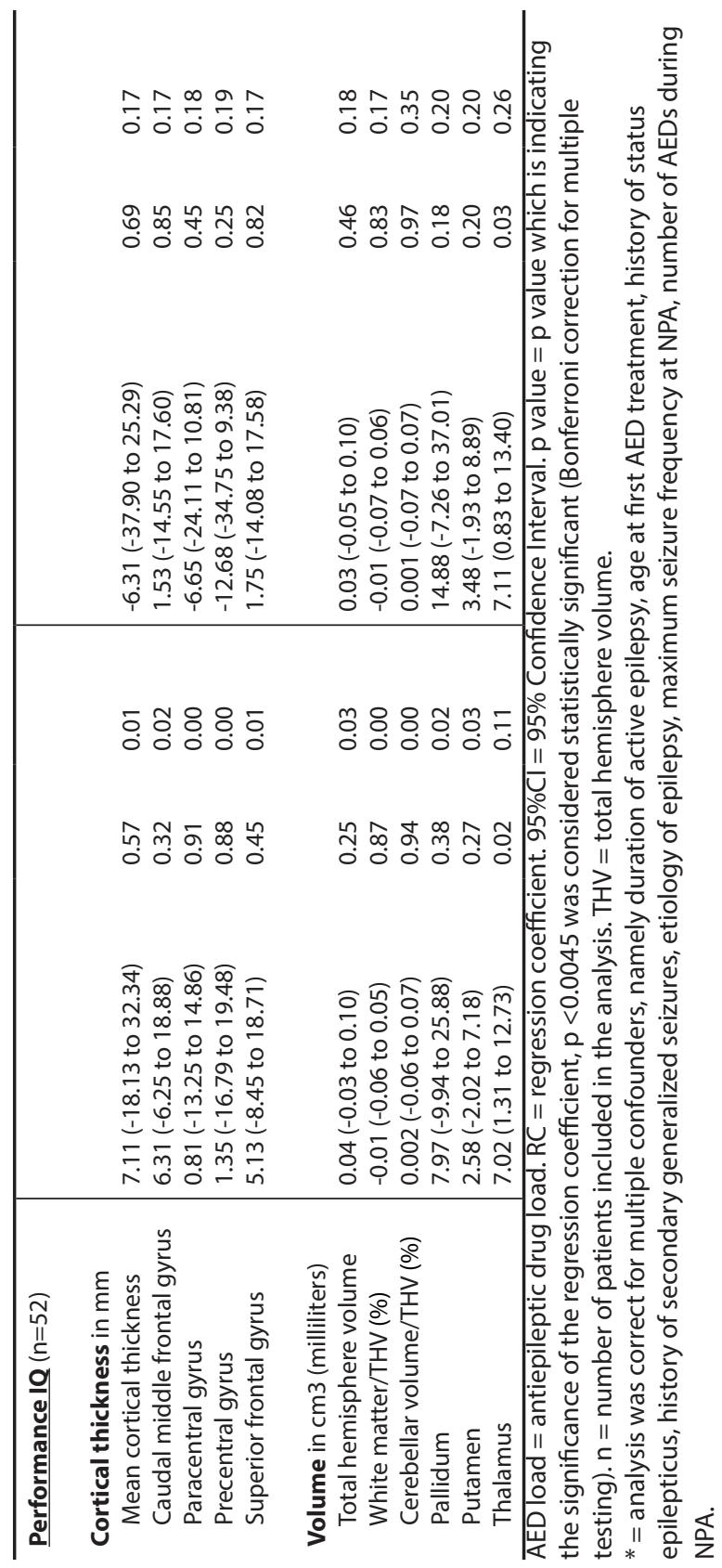




\section{Discussion}

The main finding of this study is that higher AED load is associated with lower TIQ in children. This result remained significant after adjustment for epilepsy-related confounders, including the use of AEDs at time of neuropsychological assessment, suggesting that the observed association is explained by previous cumulative drug load itself. A similar, yet non-significant association for VIQ and PIQ was observed. No significant association was found between drug load and cortical thickness or brain volume of the unaffected hemisphere, nor between cortical thickness or brain volume of the unaffected hemisphere and IQ. To indicate the clinical implication of our results, the median drug load in the study population was 5.3 drug-years, which would translate to an average TIQ reduction of 6.2 points ( $95 \% \mathrm{Cl}-10.9$ to -1.4$)$, which is not negligible in children.

Whereas most studies have focused on direct cognitive side effects of AEDs (Aldenkamp et al. 2016; Lagae 2017, 2006; Bath and Scharfman 2013; Loring and Meador 2004), the longterm effects of AED exposure on cognitive functioning remain largely unknown. Farwell and colleagues studied the long-term effects of an AED on IQ in children, in a relatively healthy cohort of children with febrile seizures (Farwell et al. 1990). In this trial 217 patients with febrile seizures, aged 8 to 36 months, were randomized to a phenobarbital or placebo treatment arm and followed for two years. At the end of the trial the average IQ score in the phenobarbital arm was 8.4 points lower than in the placebo group $(95 \% \mathrm{Cl}-13.3$ to -3.5$)$. After discontinuation of the trial drugs, the difference was still $5.2 \mathrm{IQ}$ points $(95 \% \mathrm{Cl}-10.5$ to 0.04$)$, and $3-5$ years after the trial the children from the phenobarbital group scored lower on a Wide Range Achievement Test (Sulzbacher et al. 1999), although there was no significant difference in average group IQ anymore. Both the Farwell trial and our study are suggestive of long-term effects of AEDs on cognition, although both have limitations. In the case of the Farwell trial, results are based on intention-to-treat analyses, and many participants stopped trial medication or were lost to follow-up. Also, currently phenobarbital is not prescribed as standard treatment against epilepsy beyond the neonatal period.

Recently, the ENIGMA-epilepsy consortium investigated structural brain abnormalities in epilepsy patients and healthy controls (Whelan et al. 2018). Significantly smaller volumes of the thalamus, hippocampus, and pallidum of people with epilepsy were shown, compared to healthy controls, and reduced cortical thickness was found across seven regions bilaterally. In our study we were not able to find an association between structural changes in these regions and drug load, nor with IQ. Because our study did not find mediation of the association between drug load and IQ by changes in brain volume or cortical thickness, the pathophysiological mechanism behind the influence of AEDs on IQ remains unclear. The absence of gross volumetric MRI changes, however, certainly does not exclude subtle changes in cortical microstructure. Neurogenesis, apoptosis, synaptogenesis and pruning are all 
relevant processes for neurodevelopment (Webb, Monk, and Nelson 2001; Kaindl et al. 2006). Disturbing these developmental processes might account for cognitive deficits in humans preor postnatally exposed to AEDs (Kaindl et al. 2006). The hypothesis is that these disturbances are related to decreased neuronal activity during development, since all AEDs share this effect (Bittigau et al. 2002). Animal studies have given some insight in the possible neurotoxic effect of AEDs. In rodent studies, it has been shown that some compounds used in AED treatment trigger widespread apoptotic neurodegeneration throughout the developing brain when administered during the period of rapid brain growth (Bittigau et al. 2002). Furthermore, it was suggested that neurotransmitters can modulate proliferation of neural stem cells, neuroblasts and glioblasts, regulate migration and induce differentiation (Emerit, Riad, and Hamon 1992; Retz, Kornhuber, and Riederer 1996; Levitt et al. 1997; Nguyen et al. 2001). In this way, pharmacological agents that interfere with neurotransmission, such as AEDs, may cause permanent defects in the central nervous system. These findings in animal studies suggest a potential influence of AEDs on neurodevelopment in humans. The results of the current study seem to support the aforementioned theories, however, our results are at best circumstantial evidence and no causal conclusions may yet be drawn.

A strength of this study is that it is the first to explore long-term effects of AEDs on cognition, as previous research mostly focused on the short-term adverse cognitive effects of AEDs. Secondly, the effect was studied while adjusting for known confounders, including the number of AEDs used at the time of NPA to specifically exclude acute side effects of the AEDs. Finally, the amount of missing data was limited, despite the retrospective study design.

A causal relationship between drug load and IQ must be interpreted with caution, as this study has several limitations. The study population consisted of a selected group of children, since a patient was only included when both NPA results and 3DT1 MRI were available. However, NPA will usually be performed in children with a suspicion of cognitive deficits (in accordance with Dutch Guidelines (Nederlandse Vereniging voor Neurologie 2015)) or with severe epilepsy, possibly resulting in exclusion of less affected children from the study, leading to potential selection bias. This could have resulted in an overestimation of the relation between drug load and IQ. However, subgroup analysis of patients with $\mathrm{TIQ}^{3} 70$ still revealed a significant, negative association for drug load and TIQ, supporting the claims of the main analysis. Additionally, the prevalence of pharmacoresistance in the current study population was high. In addition, due to the retrospective study design, confounders were not measured in a standardized manner, which might have caused information bias. Results from a control group of epilepsy patients without AED treatment were unavailable, complicating correction for unknown confounders. Also, this study did not contain longitudinal follow-up results of the epilepsy patients.

As NPAs before start of drug treatment were unavailable, no longitudinal comparison at individual level was possible. Furthermore, the sample size was relatively small. In addition, 
when assessing the influence of AEDs on intelligence, neurodevelopmental stages were not considered in the analyses, due to the small sample size. Since it is known that neurodevelopment is a dynamic process with different neurological functions developing at different stages and timespans (Korkman et al. 2013), age might be an effect modifier in the relation between drug load and IQ, suggesting that evaluation of the relation across different categories might be insightful. Also, no distinction was made for different AEDs or dosage, as the objective of the current study was to explore the relationship between overall AED load and IQ. Because the working mechanism of each AED is different, the effects of each drug on neurodevelopment might be very different and should ideally be studied separately or in consort with drugs using the same mechanism. As IQ-scores are a rough estimate of academic potential, IQ is less sensitive for subtle or modest neuropsychological change. Consequently, additional research on the long-term influence of AED exposure on a broader neuropsychological domain would be interesting to detect mild changes in cognition. Finally, studies investigating the influence of epileptic seizures on IQ, while correcting for AED use, are scarce. Therefore, separating the effects of long-term drug use from those of ongoing seizures remains difficult.

Altogether, the observed relation must be confirmed in a prospective study consisting of epilepsy patients representative of the whole epilepsy population, while assessing the effect of AEDs in different stages of neurodevelopment and exploring these effects for different subgroups of drugs.

\section{Conclusion}

This study revealed a significant negative relation between AED load and TIQ after adjustment for epilepsy-related confounders and the number of AEDs taken at the time of NPA. This relation was not mediated by changes in brain structure. Since the implications of the current study are considerable, confirmation of a possible causal relation between drug load and IQ in a prospective, longitudinal study design is desirable. As concluding remark, it must be mentioned that a potential effect of AEDs may very well be justified when compared to the detrimental effects of epilepsy itself and continuing seizures. 


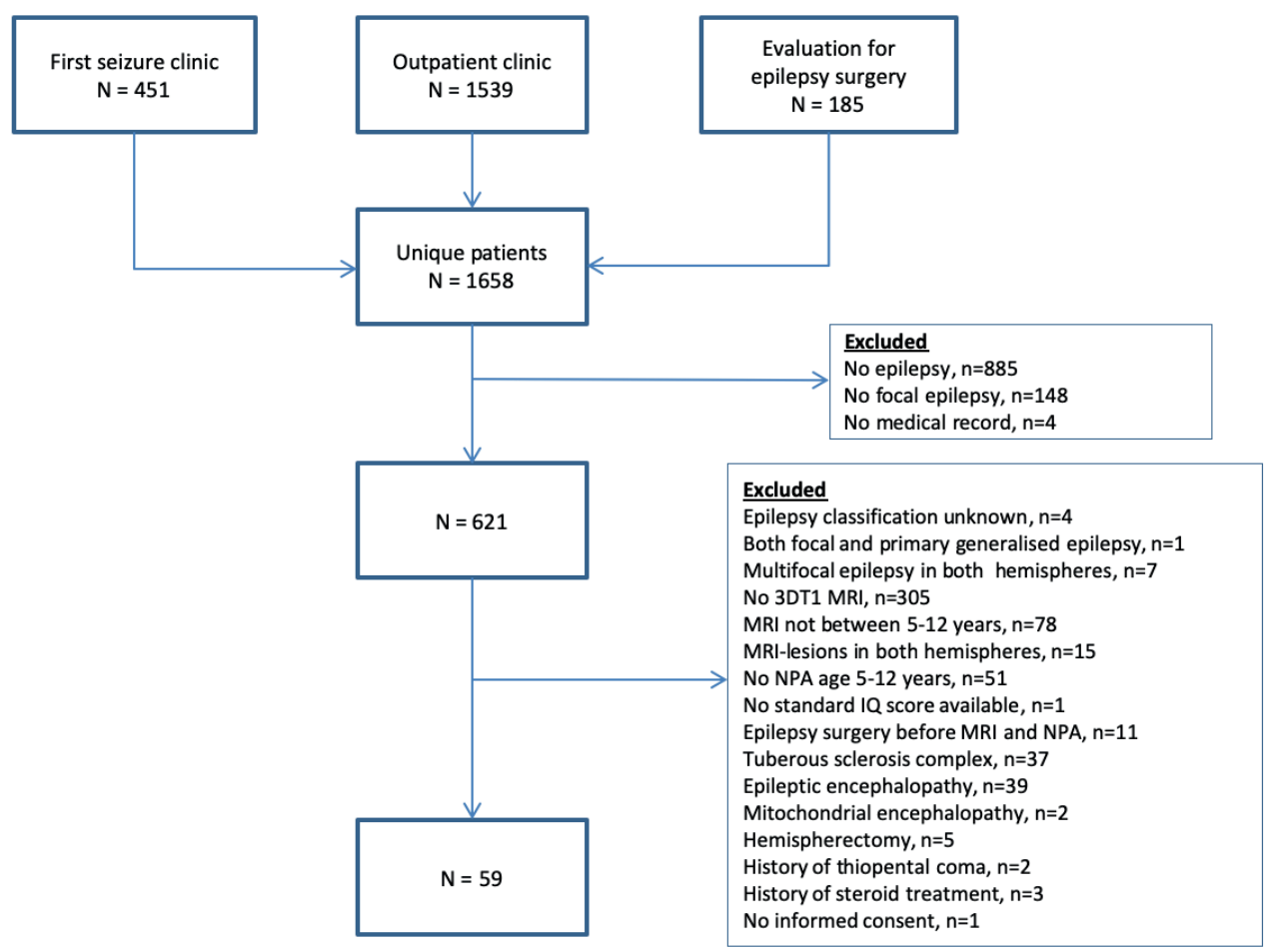

\section{Supplementary Figure 1 | Flow-chart inclusions}

Legend: $n=$ number of patients. MRI = Magnetic Resonance Imaging. 3DT1 MRI = 3DT1 modality of MRI. $\mathrm{NPA}=$ neuropsychological assessment. $\mathrm{IQ}=$ intelligence quotient. 


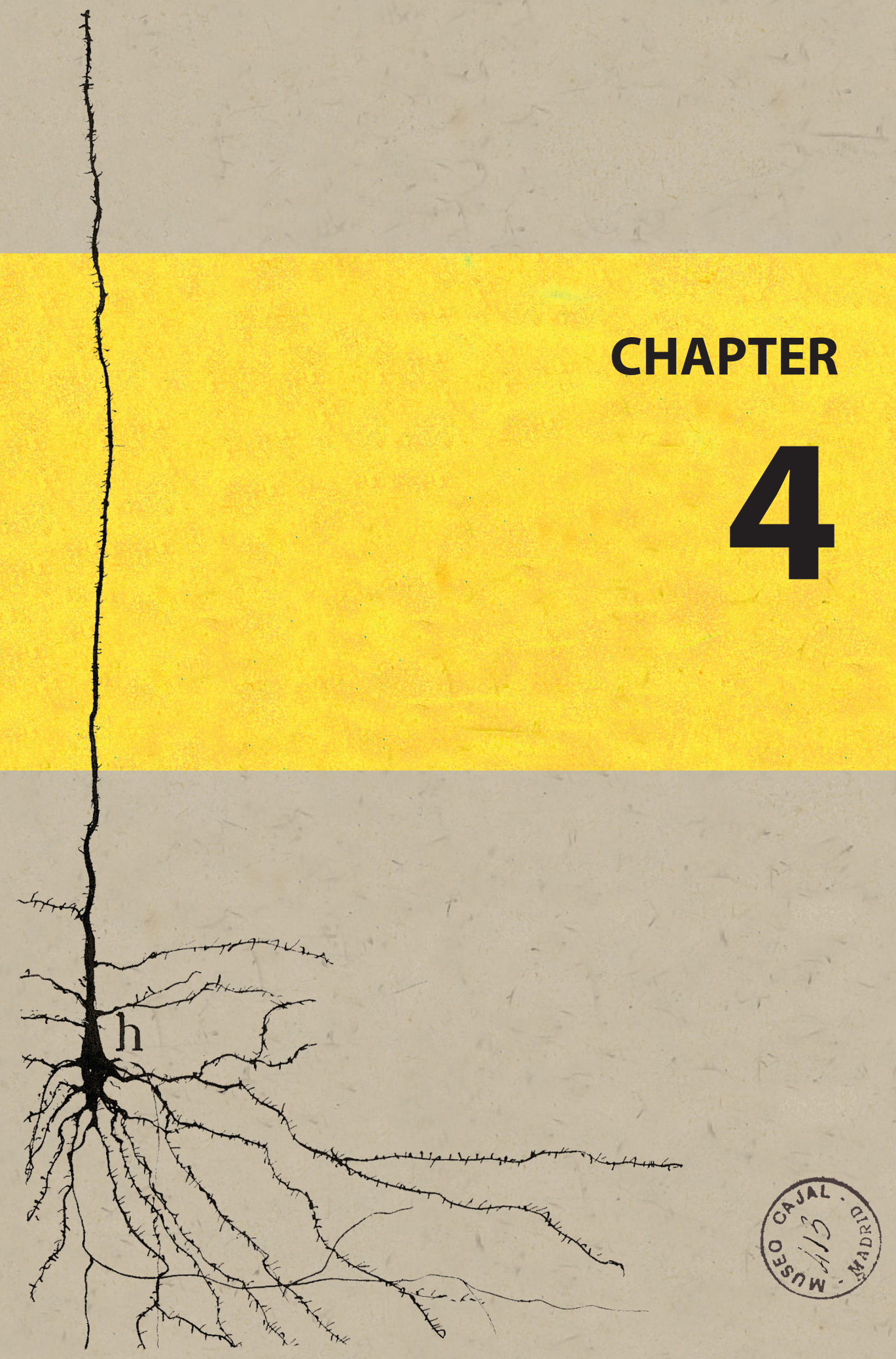




\title{
Individualised prediction model of seizure recurrence and long- term outcomes after withdrawal of antiepileptic drugs in seizure-free patients: a systematic review and individual participant data meta-analysis
}

\author{
Lamberink HJ \\ Otte WM \\ Geerts AT \\ Pavlovic M \\ Ramos-Lizana J \\ Marson AG \\ Overweg J \\ Sauma L \\ Specchio LM \\ Tennison M \\ Cardoso TMO \\ Shinnar S \\ Schmidt D \\ Geleijns K \\ Braun KPJ
}




\section{Abstract}

Background: People with epilepsy who became seizure-free while taking antiepileptic drugs might consider discontinuing their medication, with the possibility of increased quality of life because of the elimination of adverse events. The risk with this action, however, is seizure recurrence. The objectives of our study were to identify predictors of seizure recurrence and long-term seizure outcomes and to produce nomograms for estimation of individualised outcomes.

Methods: We did a systematic review and meta-analysis, and identified eligible articles and candidate predictors, using PubMed and Embase databases with a last update on Nov 6, 2014. Eligible articles had to report on cohorts of patients with epilepsy who were seizurefree and had started withdrawal of antiepileptic drugs; articles also had to contain information regarding seizure recurrences during and after withdrawal. We excluded surgical cohorts, reports with fewer than 30 patients, and reports on acute symptomatic seizures because these topics were beyond the scope of our objective. Risk of bias was assessed using the Quality in Prognosis Studies system. Data analysis was based on individual participant data. Survival curves and proportional hazards were computed. The strongest predictors were selected with backward selection. Models were converted to nomograms and a web-based tool to determine individual risks.

Findings: We identified 45 studies with 7082 patients; ten studies (22\%) with 1769 patients (25\%) were included in the meta-analysis. Median follow-up was 5.3 years (IQR 3.0-10.0, maximum 23 years). Prospective and retrospective studies and randomised controlled trials were included, covering non-selected and selected populations of both children and adults. Relapse occurred in 812 (46\%) of 1769 patients; 136 (9\%) of 1455 for whom data were available had seizures in their last year of follow-up, suggesting enduring seizure control was not regained by this timepoint. Independent predictors of seizure recurrence were epilepsy duration before remission, seizure-free interval before antiepileptic drug withdrawal, age at onset of epilepsy, history of febrile seizures, number of seizures before remission, absence of a self-limiting epilepsy syndrome, developmental delay, and epileptiform abnormality on electroencephalogram (EEG) before withdrawal. Independent predictors of seizures in the last year of follow-up were epilepsy duration before remission, seizure-free interval before antiepileptic drug withdrawal, number of antiepileptic drugs before withdrawal, female sex, family history of epilepsy, number of seizures before remission, focal seizures, and epileptiform abnormality on EEG before withdrawal. Adjusted concordance statistics were 0.65 (95\% Cl 0.65$0.66)$ for predicting seizure recurrence and $0.71(0.70-0.71)$ for predicting long-term seizure freedom. Validation was stable across the individual study populations. 
Interpretation: We present evidence-based nomograms with robust performance across populations of children and adults. The nomograms facilitate prediction of outcomes following drug withdrawal for the individual patient, including both the risk of relapse and the chance of long-term freedom from seizures. The main limitations were the absence of a control group continuing antiepileptic drug treatment and a consistent definition of long-term seizure freedom.

Funding: Epilepsiefonds. 


\section{Research in context}

Evidence before this study

We did a systematic review of the English-language scientific literature in PubMed and Embase published up to Nov 6, 2014, using the search terms "antiepileptic", "withdrawal", "recurrence", and "seizure-free", and their synonyms. Overall risk of bias for separate studies was low for study participation, study attrition, prognostic factor measurement, and outcome measurement. 25 variables were identified as significant predictors of seizure recurrence in at least one peerreviewed article. However, differences in study design, population, and methods limited the possibility to determine which were the strongest predictors, and how to combine those predictors to identify risks for the individual patient.

\section{Added value of this study}

This individual participant data meta-analysis of information from 1769 patients identified independent predictors of seizure relapse and eventual seizure freedom after antiepileptic drug withdrawal, and enabled the computation of individualised outcome risks. Our nomograms are validated across various populations and can be applied in all seizure-free patients, both children and adults, for whom antiepileptic drug withdrawal is being considered.

\section{Implications of all the available evidence}

The nomograms have the potential to improve patient consultations by providing evidencebased estimates of risk for antiepileptic drug withdrawal. Furthermore, future studies on prognostic factors for the outcome of antiepileptic drug withdrawal should correct for those identified as predictors in this paper. 


\section{Introduction}

Antiepileptic drugs suppress seizures in $65 \%$ to $85 \%$ of people with epilepsy (Shorvon and Goodridge 2013). Because of the fear of seizure relapse many people with epilepsy continue antiepileptic drug treatment even when free from seizures and despite the side-effects of the drugs. Up to $88 \%$ of patients often have several adverse effects from antiepileptic drugs (Baker et al. 1997; Perucca et al. 2009). As a result, quality of life for seizure-free patients is significantly better when antiepileptic drugs are discontinued (Sillanpää, Haataja, and Shinnar 2004), provided they remain seizure free.

Results from a meta-analysis estimated that the cumulative seizure recurrence rate after antiepileptic drug withdrawal is around 34\% (Lamberink, Otte, et al. 2015). For those who have seizure recurrence, about $80 \%$ will be able to control their seizures by reinstating antiepileptic drug treatment (Schmidt and Löscher 2005). The remaining 20\% will develop treatmentrefractory epilepsy, although there is no convincing evidence that this refractoriness occurs as a consequence of antiepileptic drug withdrawal. Nonetheless, there is some debate around whether antiepileptic drug withdrawal is safe at all (Schmidt 2011; Beghi 2011).

The dilemma between overtreatment and side-effects of antiepileptic drugs on the one hand, and the risk of seizure recurrence on the other should be considered with every seizurefree patient. However, a robust tool to guide the decision to withdraw antiepileptic drugs is not available. 25 predictors of seizure outcome have been identified, but the published populations, methods, and results were too variable to distil a definitive set of independent predictors (Lamberink, Otte, et al. 2015). Although many studies have focused on predictors of seizure recurrence, only a few have studied factors related to refractory epilepsy (Schmidt and Löscher 2005). A major limitation of prognostic meta-analyses that have used published aggregate data is that effect sizes associated with individual predictors cannot be produced because of different methods and reporting of the original studies. A method to overcome this issue is through a meta-analysis of individual participant data (IPD), in which the original data from previous studies are combined and more accurate, adjusted statistics can be computed for a large dataset (Riley, Lambert, and Abo-Zaid 2010).

In this IPD meta-analysis we aimed to identify independent predictors of seizure recurrence and long-term seizure outcome, and ultimately provide an evidence-based tool using nomograms to predict the short-term and long-term seizure outcomes for individual seizure-free patients who face the decision of whether to withdraw antiepileptic drugs. 


\section{Methods}

\section{Search strategy and selection criteria}

To select articles eligible for this study, we did a systematic search of PubMed and Embase on Nov 6, 2014 (with no date restrictions). Inclusion criteria were that they had to be original full-text articles reporting on a cohort of seizure-free patients who started antiepileptic drug withdrawal and containing information regarding seizure recurrences during and after antiepileptic drug withdrawal. We excluded surgical cohorts, reports with fewer than 30 patients, and reports on acute symptomatic seizures because these topics were beyond the scope of our objective. Unpublished data were not explored. Search queries are in Appendix 1. We checked reference lists for missed articles. Two independent researchers ( $H J L$ and KG) selected the studies and differences in article inclusion were solved through discussion. After selecting eligible articles, contact details of authors were gathered from recent articles or the internet and authors were asked to collaborate with us. A second request was sent to non-responders 6 weeks later. Authors who agreed to collaborate were requested to provide anonymous IPD for baseline, outcome, and candidate predictor variables. Aggregate data from studies for which IPD were not available were not used.

\section{Outcome and predictor variables}

We used two distinct outcome variables corresponding with the two main research questions. The first was the occurrence and timing of seizure recurrence at 2 and 5 years after initiation of antiepileptic drug withdrawal. The second was long-term seizure outcome, with favourable outcome defined as complete seizure freedom in the last year of follow-up, suggesting either no recurrence or recurrence with subsequent regain of seizure control. For those with unfavourable long-term outcome, time to event was defined as the interval between initiation of antiepileptic drug withdrawal and seizure recurrence; for seizure-free patients at last followup, irrespective of the presence of seizure recurrence, censoring time was the maximum follow-up duration.

The selection of candidate predictors was based on a systematic review of the predictors of seizure recurrence after antiepileptic drug withdrawal, 5 which identified 25 significant predictors. Three pairs of variables measured similar constructs and were therefore reduced to three single variables, resulting in a final list of 22 variables for the analysis (table 1). Information on variable definitions is in the appendix.

The quality of data presented in the original publications was previously assessed in a systematic review (Lamberink, Otte, et al. 2015) with an adjusted version of the Quality in Prognosis Studies system (Hayden, Coté, and Bombardier 2006). Potential for bias was classified as low, moderate, or high for the categories of study participation, study attrition, prognostic factor measurement, and outcome measurement. 


\section{Data analysis}

A detailed overview of statistical methods is in Appendix 2. Briefly, missing data were dealt with by multiple imputations. Random-effects proportional hazards regression was done to study prognostic factors. A selection of the strongest contributing predictors was made through backward selection of variables using the Akaike information criterion combined with manual removal of the least contributing predictors, until the most optimum model was selected. Calibration plots were created and, for validation, a concordance statistic (c statistic) was computed and adjusted for optimism by using 200 bootstrap samples. Internal-external crossvalidation (IECV) was done to assess the validity of the model across the different populations.

\section{Role of the funding source}

The funding source had no role in study design, data collection, data analysis, data interpretation, or writing of the report. The corresponding author had full access to all the data in the study and had final responsibility for the decision to submit for publication.

\section{Results}

We identified 45 reports as eligible for inclusion; 33 authors were ultimately contacted and invited to collaborate, of whom ten agreed to participate and provide IPD (Supplementary Figure 1). 1771 (25\%) of 7082 patients were included in the initial analysis. Many authors provided additional, unpublished details on the cohorts, such as longer follow-up durations. No important issues that could compromise the analysis were identified in checking IPD from contributing cohorts. The cohorts consisted of a variety of populations (table2) (Cardoso, Cendes, and Guerreiro 2003; Geerts et al. 2005; MRC AED withdrawal study group 1991; Overweg et al. 1987; Pavlović, Jović, and Pekmezović 2012; Ramos-Lizana et al. 2010; Serra, Montenegro, and Guerreiro 2005; S Shinnar et al. 1994; L M Specchio et al. 2002; Tennison et al. 1994), some with selected populations, such as children with cryptogenic focal epilepsies (Pavlović, Jović, and Pekmezović 2012), patients only on monotherapy (L M Specchio et al. 2002), and patients older than 13 years on monotherapy with exclusion of idiopathic generalised epilepsies (Cardoso, Cendes, and Guerreiro 2003), and others with mostly unselected populations of children (Geerts et al. 2005; Ramos-Lizana et al. 2010; Serra, Montenegro, and Guerreiro 2005; S Shinnar et al. 1994; Tennison et al. 1994), adults (Overweg et al. 1987), or both (MRC AED withdrawal study group 1991). The maximum follow-up after start of antiepileptic drug withdrawal was 23 years (median 5.3, IQR 3.0-10.0) and for the patients with a seizure recurrence the follow-up after the recurrence was a median of 3.7 years (range $0-20 \cdot 0$, IQR 1.0-7.0). The median time to antiepileptic drug withdrawal after the last seizure was 33 months (range 3-385, IQR 24-48). 


\begin{tabular}{|c|c|c|c|c|c|}
\hline \multirow[b]{2}{*}{ Variable } & \multirow[b]{2}{*}{$n(\%)^{\ddagger}$} & \multicolumn{2}{|l|}{$\begin{array}{l}\text { seizure } \\
\text { recurrence* }\end{array}$} & \multicolumn{2}{|c|}{$\begin{array}{l}\text { seizures in last year of follow- } \\
\text { up }^{+}\end{array}$} \\
\hline & & HR $(95 \% \mathrm{Cl})$ & p-value & HR $(95 \% \mathrm{Cl})$ & p-value \\
\hline Female sex & $842(48 \%)$ & $1.08(0.94-1.24)$ & 0.2745 & $1.43(1.02-2.01)$ & 0.0391 \\
\hline \multicolumn{6}{|l|}{ Age at onset epilepsy } \\
\hline Childhood age at onset (0-10) & $1087(61 \%)$ & $0.75(0.60-0.92)$ & 0.0064 & $1.31(0.80-2.16)$ & 0.3069 \\
\hline Adolescent age at onset (11-17) & $387(22 \%)$ & $1.15(0.93-1.42)$ & 0.2008 & $1.41(0.84-2.36)$ & 0.1769 \\
\hline Adult age at onset $(\geq 18)$ & $295(17 \%)$ & Reference & & Reference & \\
\hline Age at withdrawal in years ${ }^{\S}$ & $15(0-84)$ & $1.01(1.01-1.02)$ & $<0.0001$ & $1.00(0.98-1.01)$ & 0.5155 \\
\hline Family history of epilepsy & $365 / 1735$ (21\%) & $1.16(0.98-1.38)$ & 0.0828 & $1.55(1.04-2.30)$ & 0.0311 \\
\hline History of neonatal seizures & $53 / 1601(3 \%)$ & $1.30(0.91-1.84)$ & $0 \cdot 1440$ & $1.77(0.77-4.07)$ & 0.1792 \\
\hline History of febrile seizures & 199/1765 (11\%) & $1.27(1.03-1.56)$ & 0.0250 & $1.06(0.61-1.85)$ & 0.8424 \\
\hline$\geq 10$ seizures before remission & $573 / 1446(40 \%)$ & $1.52(1.29-1.81)$ & $<0.0001$ & $2 \cdot 21(1 \cdot 15-3.37)$ & 0.0003 \\
\hline $\begin{array}{l}\text { Epilepsy duration before remission } \\
\text { in years }{ }^{\S}\end{array}$ & $1(0-5)$ & $1.04(1.03-1.05)$ & $<0.0001$ & $1.03(1.01-1.06)$ & 0.0118 \\
\hline $\begin{array}{l}\text { Seizure free interval before AED } \\
\text { withdrawal in years }{ }^{\S}\end{array}$ & $3(2-4)$ & $0.94(0.91-0.98)$ & 0.0022 & $0.85(0.76-0.95)$ & 0.0057 \\
\hline Number of AEDs before withdrawal ${ }^{\S}$ & $1(1-2)$ & $1.15(1.05-1.26)$ & 0.0035 & $1.51(1.24-1.83)$ & $<0.0001$ \\
\hline Failure of previous AED withdrawal & $126 / 1246(10 \%)$ & $1.13(0.89-1.44)$ & 0.3268 & $1.15(0.68-1.95)$ & 0.5954 \\
\hline Focal seizures & $833 / 1652(50 \%)$ & $1.13(0.97-1.32)$ & 0.1162 & $1.81(1.26-2 \cdot 56)$ & 0.0015 \\
\hline Generalised tonic-clonic seizures & $1141 / 1652(69 \%)$ & $1.51(1.25-1.83)$ & $<0.0001$ & $1.07(0.69-1.66)$ & 0.7470 \\
\hline Multiple seizure types & $254 / 1089(23 \%)$ & $1.24(1.02-1.51)$ & 0.0334 & $0.94(0.55-1.59)$ & 0.8088 \\
\hline Remote symptomatic aetiology & $468 / 1649(28 \%)$ & $1.45(1.24-1.70)$ & $<0.0001$ & $1.80(1.26-2.56)$ & 0.0011 \\
\hline Self-limiting epilepsy syndrome $\mathrm{e}^{\bigotimes}$ & $183 / 978(19 \%)$ & $0.51(0.39-0.68)$ & $<0.0001$ & $0.48(0.25-0.92)$ & 0.0266 \\
\hline History of epileptic encephalopathy & $24 / 1142(2 \%)$ & $0.82(0.60-1.12)$ & 0.2201 & $0.79(0.29-2.12)$ & 0.6365 \\
\hline Juvenile myoclonic epilepsy & $30 / 978(3 \%)$ & $1.27(0.87-1.86)$ & 0.2116 & $0.91(0.29-2.87)$ & 0.8663 \\
\hline Developmental delay & $262 / 1742(15 \%)$ & $1.52(1.27-1.82)$ & $<0.0001$ & $1.30(0.82-2.04)$ & 0.2622 \\
\hline Motor deficit & $163 / 1736(9 \%)$ & $1.23(0.97-1.54)$ & 0.0850 & $0.90(0.47-1.72)$ & 0.7515 \\
\hline \multicolumn{6}{|l|}{ Imaging } \\
\hline Normal & $774 / 984(73 \%)$ & Reference & & Reference & \\
\hline Abnormal & 210/984 (20\%) & $1.32(1.08-1.62)$ & 0.0076 & $1.66(0.93-2.98)$ & 0.0877 \\
\hline Not performed & 77/984 (7\%) & $0.86(0.66-1.13)$ & 0.2861 & $0.71(0.37-1.36)$ & 0.2996 \\
\hline \multicolumn{6}{|l|}{ EEG before withdrawal } \\
\hline Normal & $1207 / 1490(79 \%)$ & Reference & & Reference & \\
\hline Epileptiform abnormality & $283 / 1490(18 \%)$ & $1.50(1.25-1.79)$ & $<0.0001$ & $1.68(1.11-2.54)$ & 0.0144 \\
\hline Not performed & $46 / 1490(3 \%)$ & $0.71(0.39-1.27)$ & 0.2446 & $1.14(0.27-4.78)$ & 0.8562 \\
\hline
\end{tabular}

Data are $\mathrm{n}(\%)$, median (IQR), or hazard ratios (HR [95\% CI]). Analysis was done with proportional hazards regression that included a random-effects term to correct for heterogeneity between populations.

* Based on available information before imputations; the denominator indicates total number of complete cases. 
Seizure recurrence occurred in 812 patients (46\%, table 2). Figure 1 shows the survival curve for time to seizure recurrence, with an ultimate Kaplan-Meier estimate of $48 \%$ seizure-free patients. The last seizure recurrence was 13 years after starting antiepileptic drug withdrawal. Supplementary Figure 2 shows the survival curve split by electroencephalogram (EEG) results. The overall recurrence rate was higher than the average reported in the literature (Lamberink, Otte, et al. 2015); when only published data were considered, the median of published seizure recurrence estimates of the ten included papers was 40\% (IQR 36-48), whereas the median of the 35 papers that were not included was $28 \%$ (22-37; Supplementary Table 1). Another difference between included and excluded papers was the high percentage of randomised controlled trials in the current analysis (50\%) compared with $11 \%$ in non-included papers (Supplementary Table 1). 136 (9\%) of 1455 patients were not seizure-free in the last year of follow-up (table 2), although some of those patients might have had a period of seizure freedom before this point. Of the patients with seizure recurrence and maximum follow-up between 1 and 5 years after recurrence, 202 (72\%) of 280 were seizure-free in the last year of follow-up. The proportions of patients with seizure recurrence who were seizure free at final follow-up were 121 (80\%) of 152 patients, 65 (81\%) of 80 , and 50 (88\%) of 57 for the maximum follow-up durations of 5-10 years, 10-15 years, and more than 15 years after seizure recurrence, respectively.

Seizure freedom after initiation AED withdrawal

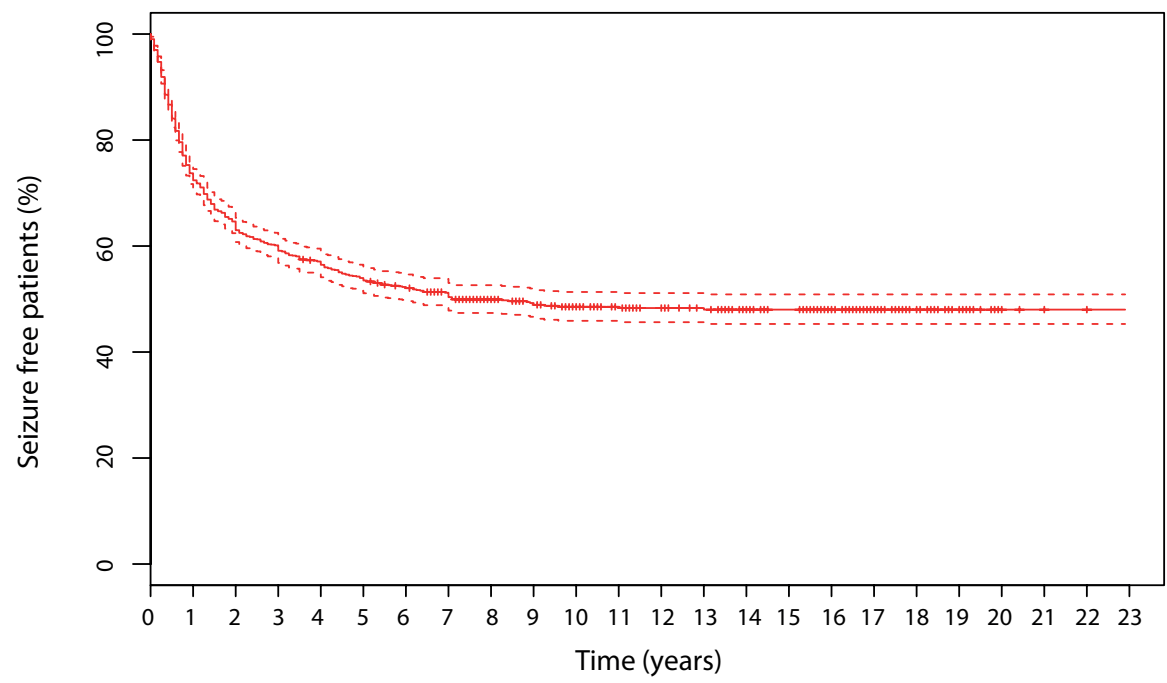

Number at risk

Cumulative $n$ events

Kaplan-Meier estimate $(95 \% \mathrm{Cl})$

$\begin{array}{cc}262 & 163 \\ 809 & 811 \\ 0.49 & 0.48 \\ (0.46-0.51) & (0.45-0.51)\end{array}$

163
811
0.48
$05-0.51)$ $0.74)(0.65)$

Figure 1 | Seizure-free patients after initiation of antiepileptic drug withdrawal

Survival curve of seizure-free patients over time with Kaplan-Meier estimates at 1, 2, 5, 10, and 13 years (time of last event in this dataset), with seizure recurrence taken as event. Time 0 equals the start of antiepileptic drug withdrawal. 
Table 2 | Characteristics of individuals from included studies

\begin{tabular}{|c|c|c|c|c|c|}
\hline & $\begin{array}{l}\text { Total } \\
(n=1769)\end{array}$ & $\begin{array}{l}\text { Cardoso } \\
(n=99)\end{array}$ & $\begin{array}{l}\text { Geerts } \\
(n=133)\end{array}$ & $\begin{array}{l}\text { MRC } \\
(n=510)\end{array}$ & $\begin{array}{l}\text { Overweg } \\
(n=65)\end{array}$ \\
\hline Year publication & - & 2003 & 2005 & 1991 & 1987 \\
\hline Study design & - & $\mathrm{RCT}^{*}$ & $\mathrm{RCT}^{\dagger}$ & $\mathrm{RCT}^{\ddagger}$ & pros \\
\hline Country & - & Brazil & $\begin{array}{l}\text { The } \\
\text { Netherlands }\end{array}$ & $\begin{array}{l}\text { United } \\
\text { Kingdom }\end{array}$ & The Netherlands \\
\hline $\begin{array}{l}\text { Follow-up in years, } \\
\text { median (IQR) }\end{array}$ & $5 \cdot 3(3-10)$ & $9 \cdot 0(7 \cdot 3-13 \cdot 3)$ & $10 \cdot 9(10-11 \cdot 3)$ & $5 \cdot 0(4-5.9)$ & $3 \cdot 0(2 \cdot 7-3 \cdot 3)$ \\
\hline $\begin{array}{l}\text { Follow-up after seizure } \\
\text { recurrence in years, } \\
\text { median (IQR) }\end{array}$ & $3 \cdot 7(0 \cdot 6-6 \cdot 8)$ & $7 \cdot 8(5.4-11 \cdot 9)$ & $9.4(7 \cdot 7-10 \cdot 4)$ & $3.9(2 \cdot 6-4.9)$ & $2 \cdot 5(1.8-2 \cdot 8)$ \\
\hline Female sex, $\%$ & $842(48 \%)$ & $46(46 \%)$ & 69 (52\%) & $260(51 \%)$ & $25(38 \%)$ \\
\hline Polytherapy, \% & $464 / 1753(26 \%)$ & 47 (47\%) & $22(17 \%)$ & $86(17 \%)$ & $46(71 \%)$ \\
\hline $\begin{array}{l}\text { Aetiology, remote } \\
\text { symptomatic, } \%\end{array}$ & $468 / 1649$ (28\%) & $72(73 \%)$ & $42(32 \%)$ & $134(26 \%)$ & $13(20 \%)$ \\
\hline $\begin{array}{l}\text { Age at onset epilepsy } \\
\text { in years, median (IQR) }\end{array}$ & $8(4-14)$ & $14(9-22)$ & $7(4-10)$ & $14(7-24)$ & $10(6-14)$ \\
\hline $\begin{array}{l}\text { Age at withdrawal in } \\
\text { years, median }(\mathrm{IQR}) \|\end{array}$ & $15(10-26)$ & $26(21-36)$ & $8(5-11)$ & $27(18-43)$ & $29(22-24)$ \\
\hline $\begin{array}{l}\text { Epilepsy duration } \\
\text { before remission in } \\
\text { months, median (IQR) }\end{array}$ & $23(4-72)$ & $8(3-15)$ & $19(5-43)$ & $53(10-143)$ & $157(94-240)$ \\
\hline $\begin{array}{l}\text { Seizure-free interval } \\
\text { before AED withdrawal } \\
\text { in months, median } \\
\text { (IQR) }\end{array}$ & $33(24-48)$ & $40(27-38)$ & $6(6-12)$ & $41(29-70)$ & $63(48-85)$ \\
\hline $\begin{array}{l}\text { Previously failed } \\
\text { withdrawal attempt, } \\
\%^{* *}\end{array}$ & $126 / 1246(10 \%)$ & $27 / 99(27 \%)$ & $0 / 133$ & $60 / 510(12 \%)$ & $4 / 51(8 \%)$ \\
\hline $\begin{array}{l}\text { Epileptiform EEG } \\
\text { before withdrawal, \%** }\end{array}$ & $283 / 1536(18 \%)$ & $12 / 99$ (12\%) & $72 / 133(54 \%)$ & $85 / 457(19 \%)$ & $31 / 64(48 \%)$ \\
\hline \multicolumn{6}{|l|}{ Seizure outcome } \\
\hline $\begin{array}{l}\text { Number of } \\
\text { recurrences, \% }\end{array}$ & $812(46 \%)$ & $53(54 \%)$ & 71 (53\%) & $235(46 \%)$ & $39(60 \%)$ \\
\hline $\begin{array}{l}\text { Seizures in last year of } \\
\text { follow-up, \%** }\end{array}$ & $136 / 1455$ (9\%) & 16/98 (16\%) & $17 / 129(14 \%)$ & $60 / 495(12 \%)$ & $12 / 60(20 \%)$ \\
\hline
\end{tabular}

Data are $\mathrm{n}(\%)$ or median (IQR). RCT=randomised controlled trial. .. =not applicable.

* Randomly assigned to either reduction of antiepileptic drugs or complete discontinuation of antiepileptic drugs. Seizure-free patients from the reduction group later completely withdrew from antiepileptic drugs. † Randomly assigned to withdrawal of antiepileptic drugs after either 6 or 12 months of freedom from seizures. ₹ Patients in this study were randomly assigned to either withdrawal or no withdrawal from antiepileptic drugs; in this analysis, we included only patients assigned to withdrawal.

$\S$ Randomly assigned to tapering from antiepileptic drugs for a duration of either 1 month or 6 months.

I Randomly assigned to tapering from antiepileptic drugs for a duration of either 6 weeks or 9 months.

II 988 patients (56\%) were younger than 18 years at withdrawal.

** Incomplete cases; the denominator shows the number of patients with available information

†† In most cases follow-up was ceased after seizure recurrence, so that the final seizure outcome could not be reported. 


\begin{tabular}{|c|c|c|c|c|c|}
\hline $\begin{array}{l}\text { Pavlovic } \\
(n=52)\end{array}$ & $\begin{array}{l}\text { Ramos-Lizana } \\
(n=216)\end{array}$ & $\begin{array}{l}\text { Serra } \\
(n=57)\end{array}$ & $\begin{array}{l}\text { Shinnar } \\
(n=264)\end{array}$ & $\begin{array}{l}\text { Specchio } \\
(n=256)\end{array}$ & $\begin{array}{l}\text { Tennison } \\
(\mathrm{n}=119)\end{array}$ \\
\hline 2012 & 2010 & 2005 & 1994 & 2002 & 1994 \\
\hline retro & pros & $\mathrm{RCT}^{\S}$ & pros & pros & $\mathrm{RCT}^{\pi}$ \\
\hline Serbia & Spain & Brazil & $\begin{array}{l}\text { United States of } \\
\text { America }\end{array}$ & Italy & $\begin{array}{l}\text { United States } \\
\text { of America }\end{array}$ \\
\hline $3 \cdot 0(2-6)$ & $4.5(2 \cdot 3-7 \cdot 5)$ & $2 \cdot 3(1 \cdot 8-5)$ & $16 \cdot 1(15 \cdot 3-17 \cdot 7)$ & $2 \cdot 0(0.7-4)$ & $2 \cdot 4(1 \cdot 3-4 \cdot 2)$ \\
\hline $2 \cdot 0(1-5 \cdot 2)$ & $4.0(2.6-5.9)$ & $3.9(2 \cdot 2-5 \cdot 7)$ & $14.8(11.3-16.9)$ & $0(0-0)$ & $0(0-1 \cdot 8)$ \\
\hline $16(31 \%)$ & $96(44 \%)$ & 25 (44\%) & $128(48 \%)$ & $130(51 \%)$ & 47 (39\%) \\
\hline $10(19 \%)$ & 40 (19\%) & $29(51 \%)$ & $13(5 \%)$ & $130(51 \%)$ & $41(34 \%)$ \\
\hline $0(0 \%)$ & $55(25 \%)$ & $18(32 \%)$ & 97 (37\%) & $37(14 \%)$ & missing \\
\hline $7(5-9)$ & $5(1-8)$ & $4(1-8)$ & $5(2-9)$ & $12(7-17)$ & $3(1-6)$ \\
\hline $14(11-16)$ & $8(4-10)$ & $10(7-13)$ & $12(8-16)$ & $22(17-30)$ & $11(8-14)$ \\
\hline $1(0-12)$ & $0(0-6)$ & $23(10-46)$ & $21(5-55)$ & $47(11-108)$ & $35(12-74)$ \\
\hline $48(36-60)$ & $25(23-27)$ & $24(24-33)$ & $30(26-42)$ & $36(36-60)$ & $24(24-48)$ \\
\hline $0 / 52$ & $0 / 0$ & $3 / 57(5 \%)$ & $22 / 264(8 \%)$ & $0 / 0$ & $10 / 80(13 \%)$ \\
\hline $0 / 41$ & $0 / 171$ & $0 / 41$ & $0 / 158$ & $55 / 256(21 \%)$ & $28 / 116(24 \%)$ \\
\hline $19(37 \%)$ & $56(26 \%)$ & $23(40 \%)$ & $110(42 \%)$ & $160(63 \%)$ & 46 (39\%) \\
\hline $0 / 47$ & $6 / 211(3 \%)$ & $9 / 56(16 \%)$ & $11 / 258(4 \%)$ & $5 / 101(5 \%)$ & t+ \\
\hline
\end{tabular}


Five variables had missing values in between $30 \%$ and $45 \%$ of patients (Supplementary Table 2, Supplementary Figure 3). Imputation was not possible for two cases because of too much missing information; these cases were removed from further analysis, which was thus done for 1769 patients. The risk of bias based on the published papers in the ten selected cohorts was scored as low to partly present (Supplementary Table 3) (Lamberink, Otte, et al. 2015)

Univariable predictors of seizure recurrence are presented in table 1, showing 14 significant variables. With respect to the long-term outcome, defined as the presence of seizures in the last year of follow-up, ten variables were significantly related in univariate analysis. To investigate a possible selection bias for the variable of failure of previous antiepileptic drug withdrawal, baseline characteristics between positive and negative cases were investigated; this analysis showed no large differences between the groups besides a longer duration of epilepsy (median 61 months [IQR 19-116] vs 24 months [5-68]), and a longer seizure free interval (median 41 [IQR 48-62] vs 31 months [24-48]; Supplementary Table 4) in the group of patients who had a previous relapse after withdrawal.

For the risk of seizure recurrence and the chance of long-term seizure freedom, respectively, 13 and 12 independent predictors were identified in multivariable modelling (Supplementary Tables 5 and 6). It was possible to reduce the number of variables in each model to eight without having an effect on the calibration plots or the validation statistics. The final reduced models with hazard ratios are in Supplementary Tables 7 and 8 . A visual representation of the models is in Figure 2, Figure 3, which are nomograms that can be applied for direct use in clinical practice to calculate the chance of both outcome measures at specific timepoints in each individual patient. Independent predictors of seizure recurrence were epilepsy duration before remission, seizure-free interval before antiepileptic drug withdrawal, age at onset of epilepsy, history of febrile seizures, number of seizures before remission, absence of a self-limiting epilepsy syndrome, developmental delay, and epileptiform abnormality on EEG before withdrawal. Independent predictors of seizures in the last year of follow-up were epilepsy duration before remission, seizure-free interval before antiepileptic drug withdrawal, number of antiepileptic drugs before withdrawal, female sex, family history of epilepsy, number of seizures before remission, focal seizures, and epileptiform abnormality on EEG before withdrawal. For practical purposes the nomograms were translated into a web-based tool for risk calculation.

The adjusted c statistic for predicting seizure recurrence was 0.65 (95\% $\mathrm{Cl} 0.65-0.66)$. In the validation procedure, the c statistic varied between 0.64 and 0.67 , thus showing stability across all populations (Supplementary Table 9). For predicting long-term seizure freedom, the adjusted c statistic was $0.71(0.70-0.71)$, which varied in the validation procedure between 0.68 and 0.79 (Supplementary Table 10). Lastly, plotting the predicted probabilities against the observed proportions showed good calibration for both models (Figure 2, Figure 3). 
\&

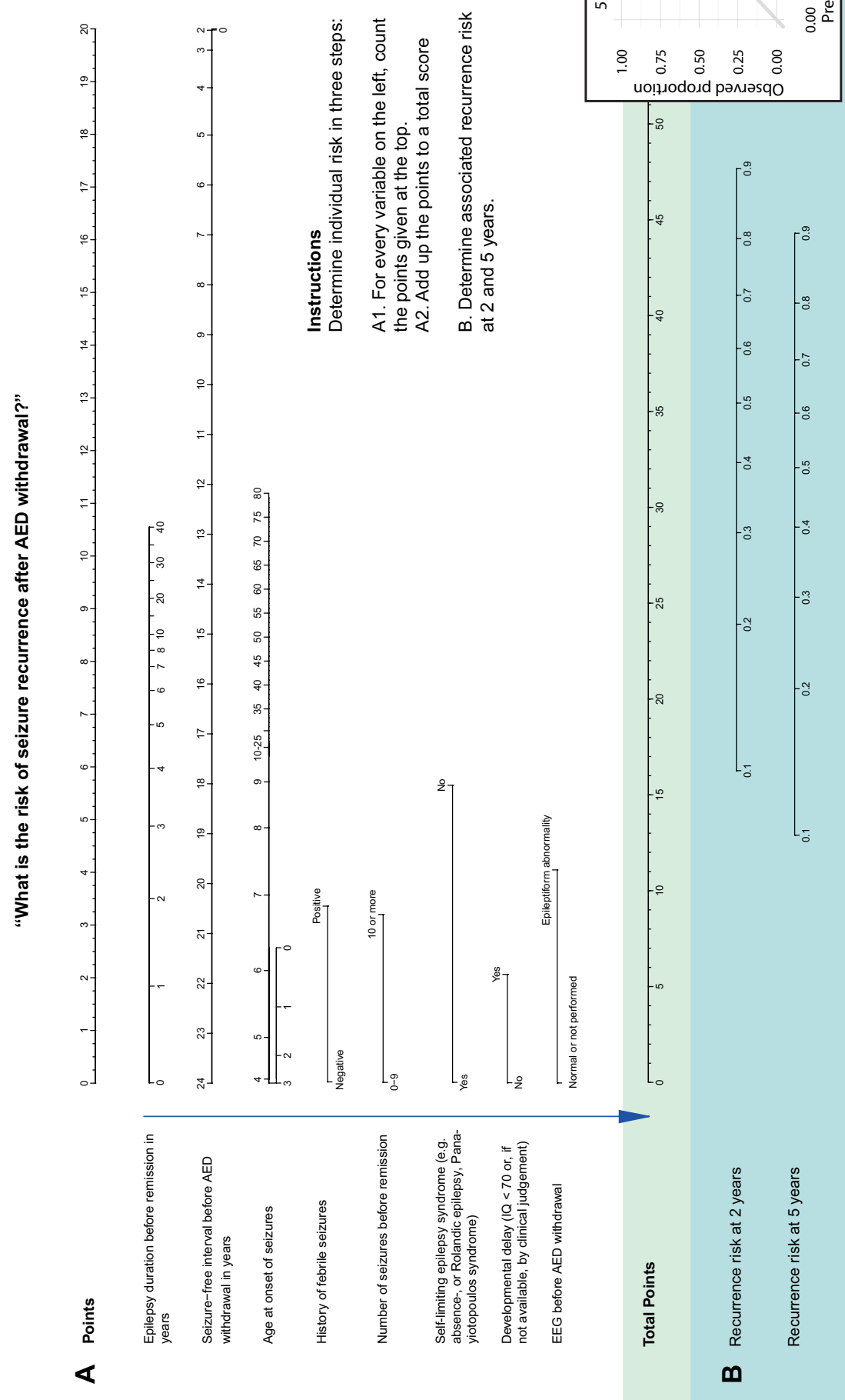

ฮั

है 它

可

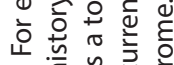
完言艺

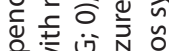

응 谣

。出出

을

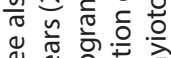

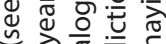

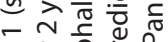

ㅎํㄴ 웡

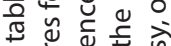

. 촌ㅎㄴ원

จ

는 뜨응 잉

है 인 즐 응

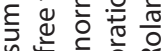

ㄴㄷㅇ

히 은

언

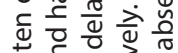

额

$\subseteq$ ष을

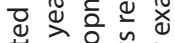

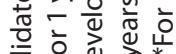



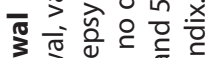

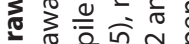

흥 这先 융

茪 至

잉

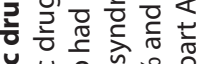

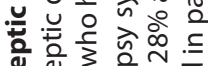

흥

웡 웡 훙

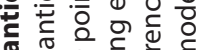

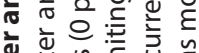

ए

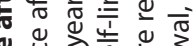

\&

凹

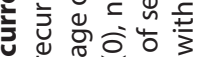

ญั



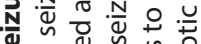

⿶凵

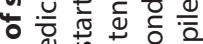

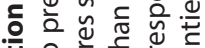
융워

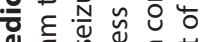

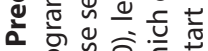
N

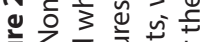

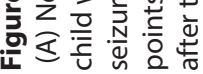


త

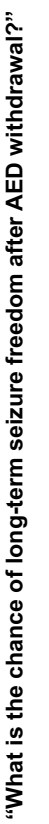

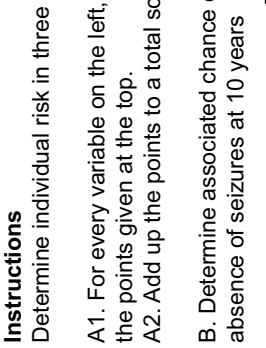
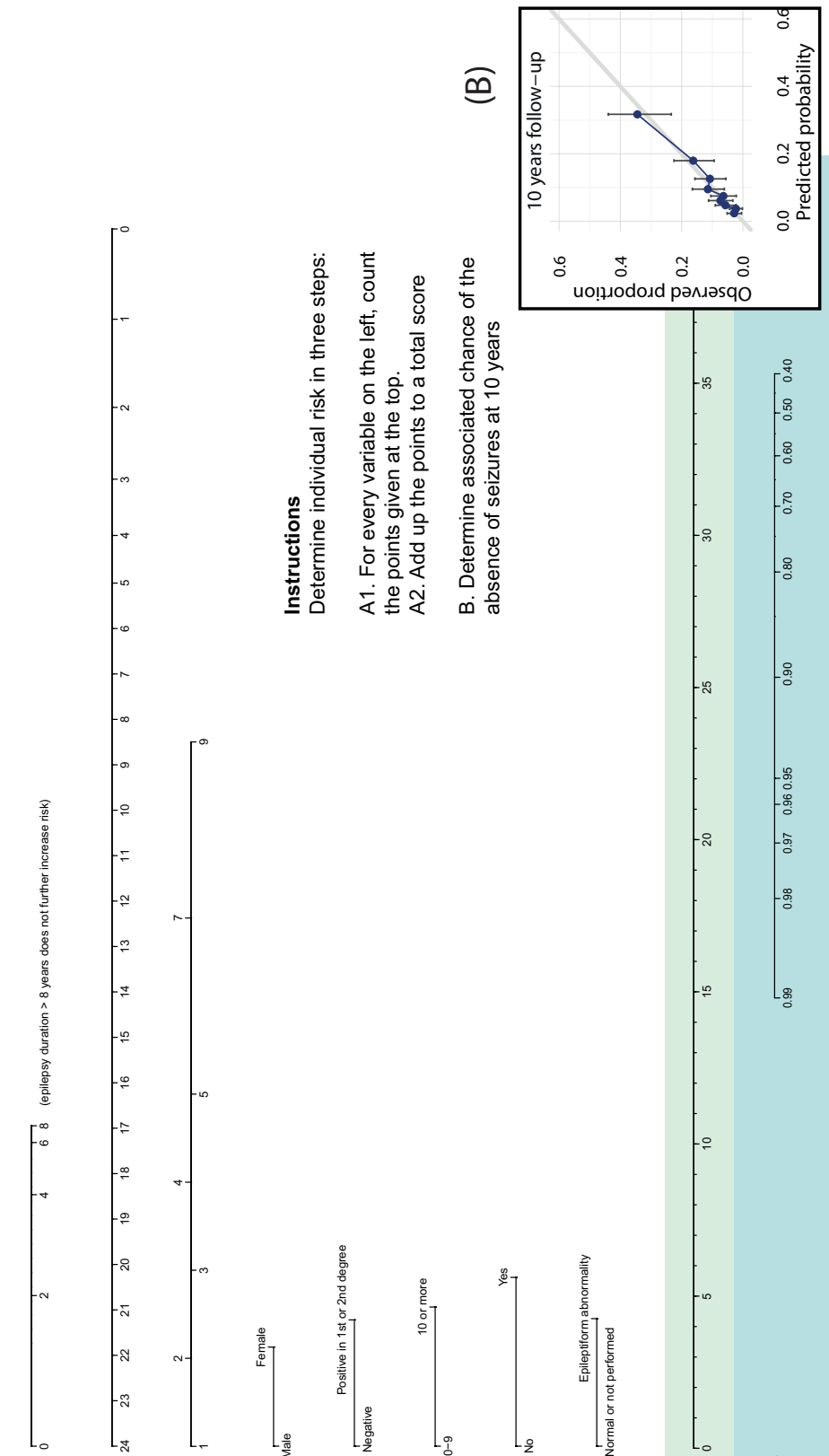

-1
+1
+1


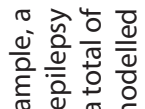


흥 눟ㅎㅎㅇ 증 $\overline{0}$ ปั

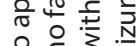

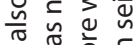
ष्ट 휸 으은

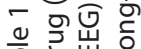
흥 늘 $\subseteq$ 둔


눈 ह 등 웡 है ㄴํํ원 든 는 능 엉 드응

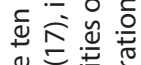

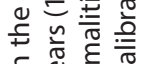
$\subseteq \stackrel{\pi}{\pi}$ 월 충 윽흔 훙 웅



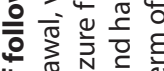

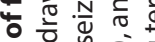
ज 응

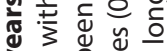
ภ원 은 능 뜬 to.



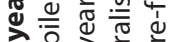
중 范䒕高 す ๘

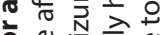


ह 언 융 氜 ข

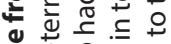
촌

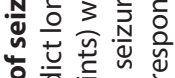
to

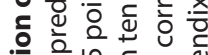
는 눙

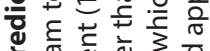
ㅎํㄴ $\bar{m} \stackrel{0}{2} \stackrel{0}{\longleftarrow}$

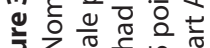

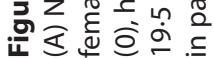




\section{Discussion}

This prognostic IPD meta-analysis of the risks of antiepileptic drug withdrawal in 1769 seizurefree people with epilepsy yielded clinically useful nomograms to predict individual seizure outcome. Relapse occurred in 812 (46\%) patients, while only 136 (9\%) of 1455 in the cohort of patients with available information had seizures in the last year of follow-up. The proportion of relapsing patients who did not regain freedom from seizures decreased with longer follow-up times. The strongest predictors included in the nomograms for seizure recurrence were duration of epilepsy, duration of the seizure-free interval, age at onset of seizures, history of febrile seizures, ten or more seizures before remission, the absence of a self-limiting epilepsy syndrome (such as absence or Rolandic epilepsy or Panayiotopoulos syndrome), intelligence quotient (IQ) below 70, and epileptiform abnormality on EEG before antiepileptic drug withdrawal. For predicting long-term seizure outcome, the eight selected independent predictors were duration of epilepsy, duration of the seizure-free interval, number of antiepileptic drugs before withdrawal, female sex, family history of epilepsy in first or second degree relatives, ten or more seizures before remission, the presence of focal seizures, and epileptiform abnormality on EEG before antiepileptic drug withdrawal. Validation, or assessment of how well a prediction works on data other than those on which the model was built, is arguably the most important issue in prognostic modelling (Steyerberg et al. 2013); internal-external cross validation within the available data was done through IECV (Ahmed et al. 2014) with good and stable performance across all cohorts.

Several clinically important implications can be drawn from the presented data. The first is that although the 22 candidate predictors had all been reported as significant predictors in at least one peer-reviewed article (Lamberink, Otte, et al. 2015), eight of these have now been shown to have no consistent significant association with the outcome. The most striking example is the failure of a previous attempt to withdraw from medication. In line with findings from a publication by Wolf and colleagues (Wolf 2016), seizure recurrence after previous antiepileptic drug withdrawal is not related to the outcome of a second (or third) attempt. This finding is unlikely to be the result of selection bias because none of the included cohorts excluded patients with a previous failure of antiepileptic drug withdrawal and the baseline characteristics of patients with a failed previous antiepileptic drug withdrawal attempt were very similar to patients attempting withdrawal for the first time.

Another observation is the effect of epileptiform activity on EEG before antiepileptic drug withdrawal, a factor which has been debated in the past (Stagi et al. 2016). EEG abnormalities were significantly associated with outcome, but in the absence of other predictive factors they only slightly increased the risks. EEG abnormalities alone should thus not prevent withdrawal of medication, a notion which was already stated in 1987 (Overweg et al. 1987) and is in agreement with, for example, the 2013 Italian guideline on antiepileptic drug withdrawal 
(Beghi et al. 2013)divided into four pairs, critically appraised 128 published reports and provided graded recommendations answering 15 key questions: length of the seizure-free period after treatment initiation, difference in seizure-free periods in children and adults, electroencephalography (EEG.

The age at onset of epilepsy is an important predictor for seizure recurrence but not for longterm freedom from seizures. Its association with seizure recurrence is U-shaped, with an elevated risk at birth that falls to a nadir by about age 3-4 years when it begins to rise again until age 10 years and plateaus until age 25 years; subsequently, the risk continues to rise further with older ages of onset. No clear explanation for the U-shaped relation between age at onset and seizure recurrence could be found.

The duration of the seizure-free interval is negatively correlated to both seizure outcomes. Where most studies on the timing of antiepileptic drug withdrawal assess the dichotomy of early versus late antiepileptic drug withdrawal, as meta-analysed in a Cochrane review (Strozzi et al. 2015), our analysis showed that the risk decreases with every additional year of seizure freedom. The common understanding that it is advisable to wait for at least 2 years is based on an artificial threshold and the rule should at least be complemented by adding that every added seizure-free year reduces the risk. The nomograms will provide insight into the best timing for the individual patient.

As a general caveat, in addition to likelihood of the outcome, there are many more considerations in the decision to withdraw antiepileptic drugs in seizure-free patients. When counselling patients with the use of these prediction models, a physician should be aware of the way the risks are presented as it can steer the patient towards a certain choice (Gong et al. 2013). Other factors such as the fear of losing a driver's licence or even a job (Bonnett et al. 2011), the social stigma around seizures (Jacoby, Johnson, and Chadwick 1992; Kilinç and Campbell 2008), and the quality of the patient's life (Baker et al. 1997) are important considerations. The nomograms only provide individualised statistical chances and should be applied when balancing benefits and risks within the context of all these factors.

Our models might appear to be restricted to populations with relatively high recurrence rates, with an estimated $52 \%$ of patients having seizure recurrence within 23 years after antiepileptic drug withdrawal. However, the ten included studies contained many different populations, from strictly selected to population based, with recurrence rates between $26 \%$ and $63 \%$. In the internal-external cross-validation procedure the effect of the separate populations was tested by omitting them one by one. For both the populations with low and high recurrence risks, the model performance remained stable. Therefore, the high average recurrence rate is no limitation to the generalisability of the models. 
One limitation of our analysis was that the study population contained only people who made an attempt to withdraw antiepileptic drugs, since maintaining antiepileptic drugs still carries the risk of seizure recurrence and refractory epilepsy. The findings from the only two randomised trials of antiepileptic drug withdrawal showed that continued antiepileptic drug treatment is related to 7\% seizure recurrence at 1 year after withdrawal (Lossius et al. 2008) and $22 \%$ at 2 years (MRC AED withdrawal study group 1991), compared with $15 \%$ and $41 \%$ for the withdrawal groups, respectively. The development of refractory epilepsy might not be related to antiepileptic drug withdrawal: a follow-up study of the UK Medical Research Council antiepileptic drug withdrawal trial showed no differences between the two randomisation groups in terms of seizure control after relapse (Chadwick, Taylor, and Johnson 1996).

For two predictors, few cases were provided: history of epileptic encephalopathy (24 cases) and juvenile myoclonic epilepsy (JME; 30 cases). Because of these low patient numbers it cannot be concluded that these factors are not predictors of outcome. For patients with JME, 26 (87\%) of 30 had seizure relapse but all were seizure free at last follow-up. This finding suggests that few patients can be successful at antiepileptic drug withdrawal (Höfler et al. 2014; Geithner et al. 2012). However, although most patients relapse, the eventual rate of regaining freedom from seizures is high.

A limitation of using IPD from previously executed studies is that prognostic factors can be defined differently. For the included variables, some variation in the measurement of developmental delay and the definition of epilepsy duration was noted (appendix). The selflimiting epilepsy syndromes were strictly defined in our protocol and not subject to different interpretation.

Another limitation was the quantification of long-term seizure freedom chosen in the analysis. From most studies, only two outcome measures were available: seizure recurrence and seizure status in the last year of follow-up, both dichotomised as seizures being present or not. Although the presence of seizures in the last year of follow-up does not fully cover longterm outcome, it is the most accurate approximation of seizure control after seizure recurrence currently available.

In conclusion, the presented nomograms were helpful to calculate an individualised risk of antiepileptic drug withdrawal and the chance of long-term favourable seizure outcomes. They might therefore help to guide individual-tailored choices by the physician and patient. 


\section{SUPPLEMENTARY TABLES, FIGURES, AND APPENDICES}



\section{Supplementary Figure 1 | Flow-chart article inclusion}

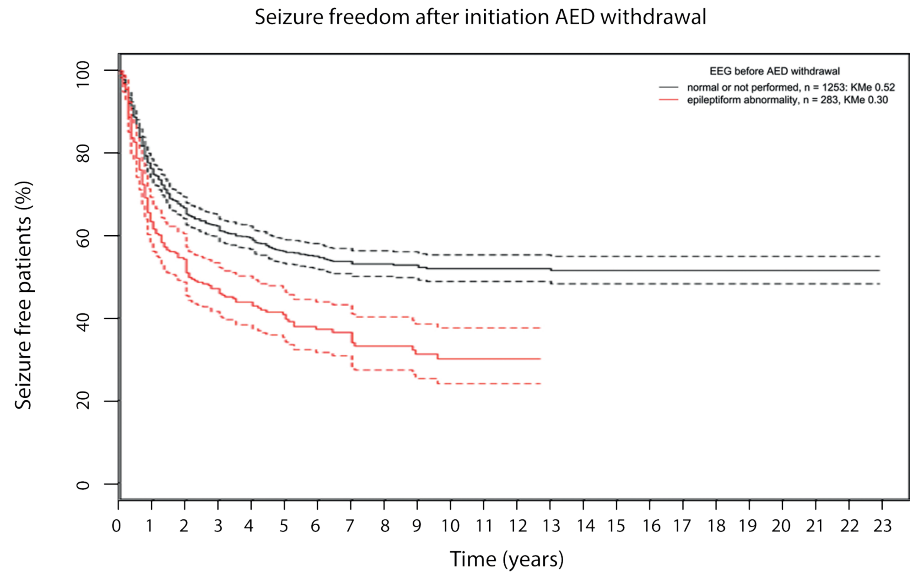

\begin{tabular}{llllll}
\cline { 2 - 5 } time in years & 0 & 1 & 2 & 5 & 10 \\
\hline $\begin{array}{l}\text { Normal or not performed, number at risk/ cu- } \\
\text { mulative number of events }\end{array}$ & 1253 & $934 / 313$ & $764 / 427$ & $406 / 513$ & $178 / 533$ \\
$\begin{array}{l}\text { Epileptiform abnormality, number at risk/ cu- } \\
\text { mulative number of events }\end{array}$ & 283 & $174 / 108$ & $147 / 137$ & $71 / 163$ & $27 / 175$
\end{tabular}

Supplementary Figure 2 | Seizure free patients after initiation of AED withdrawal, separated by EEG results before AED withdrawal.

Survival curve of seizure free patients over time with Kaplan-Meier estimates at 1, 2, 5, 10 and 13 years (time of last event in this dataset), with seizure recurrence taken as event. Time of zero equals the start of $\mathrm{AED}$ withdrawal. $\mathrm{KMe}=$ Kaplan-Meier estimate. 
Supplementary Table 1 | Comparing follow-up and seizure recurrence rate between included studies and non-participating studies

\begin{tabular}{|c|c|c|c|}
\hline Variable & $\begin{array}{l}\text { All studies ( } \mathrm{n}=45 \mathrm{co}- \\
\text { horts, } 7082 \text { patients) }\end{array}$ & $\begin{array}{l}\text { Included studies ( } \mathrm{n}=10 \\
\text { cohorts, } 17 \text { patients) }\end{array}$ & $\begin{array}{l}\text { Non-participating } \\
\text { studies ( } \mathrm{n}=35 \\
\text { cohorts, } 5311 \text { pa- } \\
\text { tients) }\end{array}$ \\
\hline $\begin{array}{l}\text { Follow-up duration in months, } \\
\text { median (range; IQR) }\end{array}$ & $\begin{array}{l}48 \\
(12-408 ; 31-66)\end{array}$ & $\begin{array}{l}44 \\
(24-129 ; 36-56)\end{array}$ & $\begin{array}{l}48 \\
(12-408 ; 30-68)\end{array}$ \\
\hline $\begin{array}{l}\text { Seizure recurrence rate, medi- } \\
\text { an (range; IQR) }\end{array}$ & $\begin{array}{l}30 \% \\
(10-66 ; 25-40)\end{array}$ & $\begin{array}{l}40 \% \\
(26-66 ; 36-48)^{*}\end{array}$ & $\begin{array}{l}28 \% \\
(10-63 ; 22-37)\end{array}$ \\
\hline $\begin{array}{l}\text { Year of publication, median } \\
\text { (range; IQR) }\end{array}$ & $\begin{array}{l}1999 \\
(1981-2014 \\
1993-2006)\end{array}$ & $\begin{array}{l}2002 \\
(1987-2012 ; \\
1994-2005)\end{array}$ & $\begin{array}{l}1999 \\
(1981-2014 \\
1992-2007)\end{array}$ \\
\hline $\begin{array}{l}\text { Ages included } \\
\text { Only adults } \\
\text { Only children } \\
\text { Both adults and children }\end{array}$ & $\begin{array}{l}2(4 \%) \\
28(62 \%) \\
15(33 \%)\end{array}$ & $\begin{array}{l}1(10 \%) \\
5(50 \%) \\
4(40 \%)\end{array}$ & $\begin{array}{l}1(3 \%) \\
23(66 \%) \\
11(31 \%)\end{array}$ \\
\hline $\begin{array}{l}\text { Type of population } \\
\text { Population based } \\
\text { Secondary care } \\
\text { Specialised centre } \\
\text { Mixed } \\
\text { Unclear }\end{array}$ & $\begin{array}{l}3(7 \%) \\
7(16 \%) \\
32(71 \%) \\
1(2 \%) \\
2(4 \%)\end{array}$ & $\begin{array}{l}1(10 \%) \\
1(10 \%) \\
7(70 \%) \\
1(10 \%) \\
0\end{array}$ & $\begin{array}{l}2(6 \%) \\
6(17 \%) \\
25(71 \%) \\
0 \\
2(6 \%)\end{array}$ \\
\hline $\begin{array}{l}\text { Design } \\
\text { Retrospective } \\
\text { Prospective } \\
\text { RCT }\end{array}$ & $\begin{array}{l}12(27 \%) \\
24(53 \%) \\
9(20 \%)\end{array}$ & $\begin{array}{l}1(10 \%) \\
4(40 \%) \\
5(50 \%)\end{array}$ & $\begin{array}{l}11(31 \%) \\
20(57 \%) \\
4(11 \%)\end{array}$ \\
\hline
\end{tabular}

$\mathrm{IQR}=$ interquartile range. $\mathrm{RCT}=$ Randomised controlled trial

*medians were calculated from data available from published results as available from our previous systematic review (Lamberink, 2015), not with the available individual patient data (IPD). Because the IPD contained longer follow-up than published results, the recurrence rate reported elsewhere in the current publication is higher. 
Supplementary Table 2 | Missing data before imputations

\begin{tabular}{lll}
\hline Variable & $\mathrm{n}$ missing values & $\%$ missing values \\
\hline Censor variable & 3 & $0 \cdot 2 \%$ \\
History of febrile seizures & 4 & $0 \cdot 2 \%$ \\
Number of AEDs. categorical & 16 & $0 \cdot 9 \%$ \\
Follow-up & 26 & $1 \cdot 5 \%$ \\
Developmental delay & 27 & $1 \cdot 5 \%$ \\
Motor deficit & 33 & $1 \cdot 9 \%$ \\
Family history of epilepsy & 34 & $1 \cdot 9 \%$ \\
EEG, general abnormalities & 53 & $3 \cdot 0 \%$ \\
Number of AEDs. numerical & 69 & $3 \cdot 9 \%$ \\
Focal seizures & 117 & $6 \cdot 6 \%$ \\
Generalised tonic-clonic seizures & 117 & $6 \cdot 6 \%$ \\
Aetiology & 120 & $6 \cdot 7 \%$ \\
History of neonatal seizures & 168 & $9 \cdot 4 \%$ \\
EEG, specific epileptiform abnormalities & 233 & $13 \cdot 1 \%$ \\
Seizure status at last follow-up & 314 & $17 \cdot 7 \%$ \\
Total number of seizures & 323 & $18 \cdot 2 \%$ \\
Failure of previous withdrawal attempt & 523 & $29 \cdot 4 \%$ \\
Epileptic encephalopathy & 627 & $35 \cdot 2 \%$ \\
Multiple seizure types & 680 & $38 \cdot 2 \%$ \\
Imaging & 708 & $39 \cdot 8 \%$ \\
Self-limiting epilepsy syndromes & 791 & $44 \cdot 5 \%$ \\
Juvenile myoclonic epilepsy & 791 & $44 \cdot 5 \%$ \\
\hline & & \\
& 33 & \\
\hline
\end{tabular}


Histogram of missing data

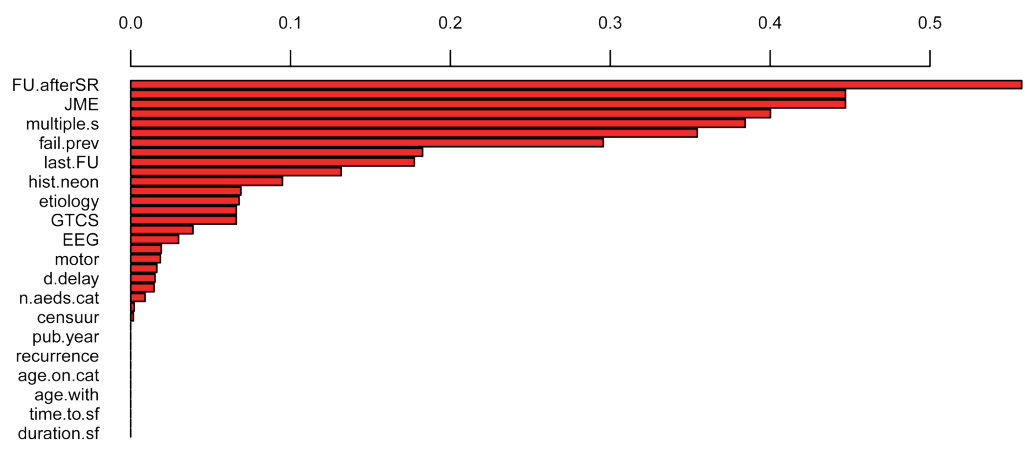

\section{Pattern}



Supplementary Figure 3 | Missing data before imputations 
Supplementary Table 3 | Quality appraisal of prognostic studies (Hayden et al., 2006)

\begin{tabular}{lllll}
\hline Publication & $\begin{array}{l}\text { Study } \\
\text { participation }\end{array}$ & $\begin{array}{l}\text { Study } \\
\text { attrition }\end{array}$ & $\begin{array}{l}\text { Prognostic factor } \\
\text { measurement }\end{array}$ & $\begin{array}{l}\text { Outcome } \\
\text { measurement }\end{array}$ \\
\hline Cardoso, 2003 & $+/-$ & $+/-$ & + & + \\
Geerts, 2005 & + & + & + & + \\
MRC, 1991 & + & + & $+/-$ & + \\
Overweg, 1987 & $+/-$ & $+/-$ & + & + \\
Pavlovic, 2012 & + & - & + & + \\
Ramos-Lizana, 2010 & + & + & + & + \\
Sauma, 2005 & $+/-$ & + & + & + \\
Shinnar, 1994 & + & + & + & + \\
Specchio, 2002 & + & - & + & + \\
Tennison, 1994 & + & + & + \\
\hline
\end{tabular}

${ }^{\prime}+{ }^{\prime}=$ bias is sufficiently limited; ${ }^{\prime} \pm^{\prime}=$ potential for bias is partly present; ${ }^{\prime}-{ }^{\prime}=$ high potential for bias.

Adapted from: Lamberink HJ, Otte WM, Geleijns K, Braun KPJ. Antiepileptic drug withdrawal in medically and surgically treated patients: a meta-analysis of seizure recurrence and systematic review of its predictors. Epileptic Disorders 2015; 17(3):211-228 with permission from John Libbey Eurotext 
Supplementary Table 4 | Baseline differences between patients with a history of seizure recurrence after AED withdrawal and those attempting for the first time

\begin{tabular}{lll}
\hline & $\begin{array}{l}\text { No previous attempt (n } \\
(1120)\end{array}$ & $\begin{array}{l}\text { Failure previous } \\
\text { attempt of AED with- } \\
\text { drawal (n= 126) }\end{array}$ \\
\hline FU in months (median, IQR) & $73(47-137)$ & $71(54-149)$ \\
Seizure recurrence & $508(45 \%)$ & $64(51 \%)$ \\
FU after seizure recurrence in months (median, IQR) & $59(33-120)$ & $60(48-118)$ \\
Seizures at last follow-up & $108 / 1016(11 \%)$ & $15 / 115(13 \%)$ \\
Sex, female & $526(47 \%)$ & $69(55 \%)$ \\
Age at onset (median, IQR) & $9(4-15)$ & $9(3-18)$ \\
Age at onset $\geq 11$ & $460(41 \%)$ & $53(42 \%)$ \\
Age at withdrawal (median, IQR) & $16(10-27)$ & $22(15-37)$ \\
Duration of epilepsy, months (median, IQR) & $24(5-68)$ & $61(19-116)$ \\
Seizure free interval, months (median, IQR) & $31(24-48)$ & $41(48-62)$ \\
Number of AEDs (median, IQR) & $1(1-1)$ & $1(1-1)$ \\
History of febrile seizures & $122 / 1116(11 \%)$ & $16(13 \%)$ \\
Family history & $249 / 1096(23 \%)$ & $37 / 123(29 \%)$ \\
$\geq$ ten seizures before remission & $320 / 834(38 \%)$ & $40 / 96(42 \%)$ \\
Benign & $69 / 481(14 \%)$ & 0 \\
Focal seizures & $526 / 1050(50 \%)$ & $65 / 116(56 \%)$ \\
Developmental delay & $185 / 1103(17 \%)$ & $10 / 125(8 \%)$ \\
EEG epileptiform abnormality & $194 / 945(21 \%)$ & $18 / 113(16 \%)$ \\
\hline FU & & \\
\hline
\end{tabular}

FU $=$ follow-up 


\section{Supplementary Table 5 | Full prediction model 1: predicting seizure recurrence}

\begin{tabular}{lll}
\hline Variable & HR $(\mathbf{9 5} \% \mathbf{C I})$ & p-value \\
\hline Epilepsy duration before remission in years & $*$ & $*$ \\
Seizure free interval before AED withdrawal in years & $*$ & $*$ \\
Age at onset of epilepsy & $*$ & $*$ \\
Family history of epilepsy & $1.22(1.03-1.45)$ & 0.0252 \\
History of febrile seizures & $1.38(1.11-1.70)$ & 0.0033 \\
$\geq 10$ seizures before remission & $1.35(1.13-1.60)$ & 0.0008 \\
Generalised tonic-clonic seizures & $1.19(0.97-1.46)$ & 0.0940 \\
Multiple seizure types & $1.13(0.90-1.42)$ & 0.2779 \\
Self-limiting epilepsy syndrome ${ }^{\dagger}$ & $0.57(0.43-0.77)$ & 0.0004 \\
History of epileptic encephalopathy & $0.69(0.50-0.93)$ & 0.0172 \\
Juvenile Myoclonic Epilepsy & $1.37(0.98-1.93)$ & 0.0664 \\
Developmental delay & $1.31(1.05-1.63)$ & 0.0160 \\
Epileptiform abnormality on EEG before withdrawal & $1.48(1.23-1.77)$ & $<0.0001$ \\
\hline
\end{tabular}

Predictors in model selected by backward selection. Adjusted c-statistic $=0.66(95 \% \mathrm{Cl} \mathrm{0.65-0.66)}$, bootstrap-corrected for $1.8 \%$ optimism

*not linearly related to outcome, no estimate available. For influence on model, see nomogram in figure 2 ; 'formerly called "benign course", e.g. absence epilepsy, benign epilepsy with centrotemporal spikes (Rolandic epilepsy), Panayiotopoulos syndrome

\section{Supplementary Table 6 | Full prediction model 2: predicting seizures in last year of follow-up}

\begin{tabular}{lll}
\hline Variable & HR & p-value \\
\hline Epilepsy duration before remission in years & $*$ & $*$ \\
Seizure free interval before AED withdrawal in years & $*$ & $*$ \\
Number of AEDs before withdrawal (per AED) & $1.34(1.09-1.64)$ & 0.0060 \\
Female sex & $1.48(1.04-2.10)$ & 0.0302 \\
Family history of epilepsy & $1.65(1.10-2.47)$ & 0.0161 \\
$\geq 10$ seizures before remission & $1.65(1.03-2.64)$ & 0.0366 \\
Remote symptomatic aetiology & $1.67(1.08-2.59)$ & 0.0219 \\
Focal seizures & $1.75(1.19-2.57)$ & 0.0042 \\
Self-limiting epilepsy syndrome ${ }^{\dagger}$ & $0.62(0.34-1.13)$ & 0.1173 \\
Juvenile myoclonic epilepsy & $1.82(0.61-5.44)$ & 0.2759 \\
Motor deficit & $0.56(0.28-1.13)$ & 0.1047 \\
Epileptiform abnormality on EEG before withdrawal & $1.68(1.11-2.54)$ & 0.0140 \\
\hline
\end{tabular}

Predictors in model selected by backward selection. Adjusted c-statistic $=0.71(95 \% \mathrm{Cl} 0.70-0.71)$, bootstrap-corrected for $3.5 \%$ optimism

*not linearly related to outcome, no estimate available. For influence on model, see nomogram in figure 4 
Supplementary Table 7 | Final prediction model 1: predicting seizure recurrence

\begin{tabular}{lll}
\hline Variable & HR $(\mathbf{9 5} \% \mathbf{C l})$ & p-value \\
\hline Epilepsy duration before remission in years & $*$ & $*$ \\
Seizure free interval before AED withdrawal in years & $*$ & $*$ \\
Age at onset of epilepsy & $*$ & $*$ \\
History of febrile seizures & $1.40(1.13-1.73)$ & 0.0020 \\
$\geq 10$ seizures before remission & $1.38(1.17-1.63)$ & 0.0002 \\
Self-limiting epilepsy syndrome $\dagger$ & $0.57(0.44-0.72)$ & $<0.0001$ \\
Developmental delay & $1.23(1.01-1.50)$ & 0.0420 \\
\hline Epileptiform abnormality on EEG before withdrawal & $1.50(1.25-1.80)$ & $<0.0001$ \\
\hline
\end{tabular}

The number of variables of the full model from supplementary table 5 was reduced. Adjusted c-statistic $=$ 0.65 (95\% Cl 0.65-0.66), bootstrap-corrected for 1.4\% optimism. Random effects 1.01.

*not linearly related to outcome, no estimate available. For influence on model, see nomogram in figure 2; tformerly called "benign course", e.g. absence epilepsy, benign epilepsy with centrotemporal spikes (Rolandic epilepsy), Panayiotopoulos syndrome

Supplementary Table 8 | Final prediction model 2: predicting seizures in last year of follow-up

\begin{tabular}{lll}
\hline Variable & HR & p-value \\
\hline Epilepsy duration before remission in years & $*$ & $*$ \\
Seizure free interval before AED withdrawal in years & $*$ & $*$ \\
Number of AEDs before withdrawal (per AED) & $1.37(1.11-1.69)$ & 0.0031 \\
Female sex & $1.42(1.01-2.01)$ & 0.0437 \\
Family history of epilepsy & $1.56(1.04-2.33)$ & 0.0302 \\
$\geq 10$ seizures before remission & $1.62(1.04-2.51)$ & 0.0315 \\
Focal seizures & $1.82(1.26-2.64)$ & 0.0016 \\
Epileptiform abnormality on EEG before withdrawal & $1.57(1.04-2.36)$ & 0.0313 \\
\hline
\end{tabular}

The number of variables of the full model from supplementary table 6 was reduced. Adjusted c-statistic $=$ 0.71 ( $95 \% \mathrm{Cl} 0.70-0.71)$, bootstrap-corrected for $3.5 \%$ optimism. Random effects 1.65 .

*not linearly related to outcome, no estimate available. For influence on model, see nomogram in figure 3 
Supplementary Table 9 | Internal-external cross-validation for prediction of seizure recurrence

\begin{tabular}{lll}
\hline Omitted study & C-statistic ( remaining studies)* & C-statistic ( omitted study )* \\
\hline Cardoso & 0.655 & 0.656 \\
Geerts & 0.645 & 0.649 \\
MRC & 0.674 & 0.665 \\
Overweg & 0.648 & 0.650 \\
Pavlovic & 0.650 & 0.652 \\
Ramos-Lizana & 0.648 & 0.638 \\
Serra & 0.646 & 0.651 \\
Shinnar & 0.643 & 0.642 \\
Specchio & 0.649 & 0.645 \\
Tennison & 0.648 & 0.649 \\
\hline
\end{tabular}

To assess the IECV, one study was omitted after which the model was fitted on the nine remaining studies. A c-statistic was computed on the nine studies, after which the model was forced onto the omitted study, where again a c-statistic was computed.

${ }^{*}$ c-statistic is corrected for optimism similar to the overall c-statistic in suppl. tables 5-8

\begin{tabular}{lll}
$\begin{array}{l}\text { Supplementary Table } \\
\text { of } \mathbf{1 0} \text { | Internal-external cross-validation for prediction of seizures in last year }\end{array}$ \\
\hline Omitted study & C-statistic ( remaining studies ) & C-statistic ( omitted study ) \\
\hline Cardoso & 0.704 & 0.700 \\
Geerts & 0.715 & 0.713 \\
MRC & 0.785 & 0.787 \\
Overweg & 0.711 & 0.714 \\
Pavlovic & 0.704 & 0.709 \\
Ramos-Lizana & 0.693 & 0.683 \\
Serra & 0.716 & 0.702 \\
Shinnar & 0.717 & 0.720 \\
Specchio & 0.716 & 0.711 \\
Tennison & 0.717 & 0.715 \\
\hline
\end{tabular}

To assess the IECV, one study was omitted after which the model was fitted on the nine remaining studies. A c-statistic was computed on the nine studies, after which the model was forced onto the omitted study, where again a c-statistic was computed.

${ }^{*}$ c-statistic is corrected for optimism similar to the overall c-statistic in suppl. tables 5-8 


\section{APPENDIX 1}

Search strategy in PubMed and EMBASE databases, as last executed until November 6, 2014

MEDLINE: (antiepileptic*[tiab] OR AED*[tiab] OR anticonvulsant*[tiab] OR anticonvulsants[MeSH]) AND (stop[tiab] OR stopping[tiab] OR reduction[tiab] OR discontinuation[tiab] OR withdrawal[tiab]) AND ("seizure free"[tiab] OR "seizure-free"[tiab] OR recurrence[tiab] OR relapse[tiab] OR remission[tiab])

EMBASE: ('anticonvulsive agent'/exp OR antiepileptic:ti:ab OR antiepileptica:ti:ab OR AED:ti:ab OR AEDs:ti:ab OR anticonvulsant:ti:ab OR anticonvulsants:ti:ab) AND ('drug withdrawal'/exp OR stop:ti:ab OR stopping:ti:ab OR reduction:ti:ab OR discontinuation:ti:ab OR withdrawal:ti:ab OR titration:ti:ab) AND ('recurrence risk'/exp OR 'seizure free':ti:ab OR 'seizure-free':ti:ab OR recurrence:ti:ab OR relapse:ti:ab OR remission:ti:ab) NOT [Medline]/lim 


\section{APPENDIX 2}

Supplementary methods

\section{Data extraction and data transformation}

Study-level data that were sought: country, type of population (population-based, secondary care, specialised centre, mixed), years of recruitment, study design.

Participant data that were requested:

1. basic information: follow-up after start of AED withdrawal in months, in case of RCT treatment allocation.

2. outcome variables: seizure recurrence, time to seizure recurrence in months, seizure status at last follow-up (presence of seizures in the last year including auras)

3. potential predictors: sex (male/female), age at onset of epilepsy (years), age at withdrawal (years), family history in $1^{\text {st }}$ or $2^{\text {nd }}$ degree, history of neonatal seizures, history of febrile seizures, number of seizures before remission (less than 10/10 or more), epilepsy duration before remission (interval between first and last seizure in months), seizure free interval before AED withdrawal (interval from last seizure to start AED withdrawal), failure of a previous AED withdrawal attempt, number of AEDs before withdrawal, presence of focal seizures, presence of generalised tonic-clonic seizures, presence of multiple seizure types, aetiology (remote symptomatic/ proven genetic/ unknown), presence of Juvenile Myoclonic Epilepsy, presence of a self-limiting epilepsy syndrome (absence epilepsy, benign epilepsy with centro-temporal spikes, Panayiotopoulos syndrome), presence or history of epileptic encephalopathy (West syndrome, Ohtahara syndrome, Lennox-Gastaut syndrome, ESES, Landau-Kleffner), developmental delay, motor deficits on neurological examination, imaging (normal, structural laesion, imaging not performed), EEG before AED withdrawal (normal, general abnormality, not performed), epileptiform EEG before AED withdrawal (normal, epileptiform abnormality, not performed).

\section{Data with variable definitions or data transformation:}

Developmental delay: IQ below 70 (4 studies, $\mathrm{n=698),} \mathrm{IQ} \mathrm{below} 70$ or clinical judgement (1 study, $n=52$ ), clinical judgement and necessity for specialised schooling ( 3 studies, $n=899$ ), IQ between 70 and 80 ( 1 study, $n=65$ ), Denver scale ( 1 study, $n=57$ ).

Imaging: many advances in imaging have come about in the past decades, resulting in a proportion of patients who have not been evaluated with MRI but with CT-scan. MRI scans have improved in accuracy over the years.

Aetiology: in 2010 the ILAE proposed a new approach to aetiology, in which the term 'remote symptomatic' is not used anymore. Since most included studies are older than the new 
definition, the old dichotomy of remote symptomatic versus not remotely symptomatic was used.

Number of AEDs: one study (Pavlovic, $\mathrm{n}=52$ ) only had binary information available, multiversus polytherapy. For the other studies, a new variable with this dichotomy was created next to the continuous variable, for the purpose of imputing the 52 missing values.

EEG abnormality: six studies provided information on both general and specific EEG abnormalities (Cardoso, Geerts, MRC, Overweg, Specchio, Tennison , $n=1182$ ). Four studies only had information on general EEG abnormalities (Pavlovic, Ramos, Serra, Shinnar, $\mathrm{n}=589$ ). For the latter four, a new variable was created in which the categories "normal EEG" and "not performed" were kept, the category "abnormal" was set to missing and imputed to create the variable "epileptiform EEG before AED withdrawal".

Epilepsy duration: two studies (Pavlovic, Ramos, $\mathrm{n}=268$ ) formulated this as the interval between start treatment and the last seizure, which was accepted as an approximation of the interval between the first and last seizure.

Self-limiting epilepsy syndromes: the definition of 'Benign childhood epilepsies' has been debated. Therefore, we chose to define this group of syndromes as including absence epilepsy, Rolandic epilepsy, or Panayiotopoulos syndrome. Patients with Juvenile Myoclonic Epilepsy are not included.

\section{Statistical analysis}

Missing information was analysed and imputed using Multiple Imputations using Chained Equations (MICE (Van Buuren and Groothuis-Oudshoorn 2011), number of imputations = 20), which provides more accurate summary statistics than deleting cases with missing data (Marshall et al. 2010). Continuous variables were imputed using predictive mean matching; logistic regression was used for imputing categorical variables. Baseline characteristics were computed before imputations were performed. If follow-up after a seizure recurrence was less than a year, the second outcome parameter could not be established, in which case the value was reported as 'missing', after which it was imputed to estimate the outcome most accurately (Vergouw et al. 2012).

To account for the variation in follow-up among participants, analyses were performed with a variation on the Cox proportional hazards model. The standard Cox model has shown disadvantages concerning heterogeneity between groups, as it can be seen as a fixedeffect model which does not adjust for between-study heterogeneity. To account for this 
heterogeneity, a shared frailty model was used, which is a random-effects extension of the Cox proportional hazards model (Therneau 2015). As an indication of heterogeneity, the randomeffects statistic reported in this paper is the approximate hazard ratio between two equally sized prognostic groups from different cohorts.

The assumption of linearity was assessed by inspection of martingale residuals and if violated corrected by restricted cubic splines with three to five knots. In the survival analyses we assumed censoring to be non-informative, and the assumption of proportional hazards was not violated as tested by statistical and visual inspection of Schoenfeld residuals (Grambsch and Therneau 1994). Multi-collinearity was checked through a correlation matrix, after which age at withdrawal was removed from the models because of collinearity with age at onset of seizures (Pearson's $r=0 \cdot 80$ ).

A selection of strongest contributing predictors was made through backward selection of variables using Akaike information criterion (AIC) combined with manual removal of least contributing predictors, until the most optimal model was selected. For the purpose of creating a prediction model, categorical variables with three categories were reduced to binary variables when the third category was not contributing to the model (e.g. instead of EEG before withdrawal categories "normal", "epileptiform abnormality" and "not performed", comparing "normal or not performed" with "epileptiform abnormality"). Using the final reduced model, a nomogram was created to assist the clinician in computing the individual risks for a patient.

To validate the models, concordance-statistics (c-statistics) were computed on the model and on 200 bootstrap samples to correct for optimism. The adjusted c-statistic and percentage of optimism are reported. Furthermore, internal-external cross-validation (IECV) was performed to assess validity of the model across the different populations. With this method, it can be shown that the models work similarly in the different populations, and it therefore functions as external validation (Ahmed et al. 2014). To assess the calibration of the models, a graphic plotting the predicted probability versus the observed proportion was used. The plotted data points should align the diagonal grey line.

Statistical analyses were performed with $\mathrm{R}$ Statistical Software version $3 \cdot 2 \cdot 2$, using packages 'MICE', 'coxme', and 'rms'. 


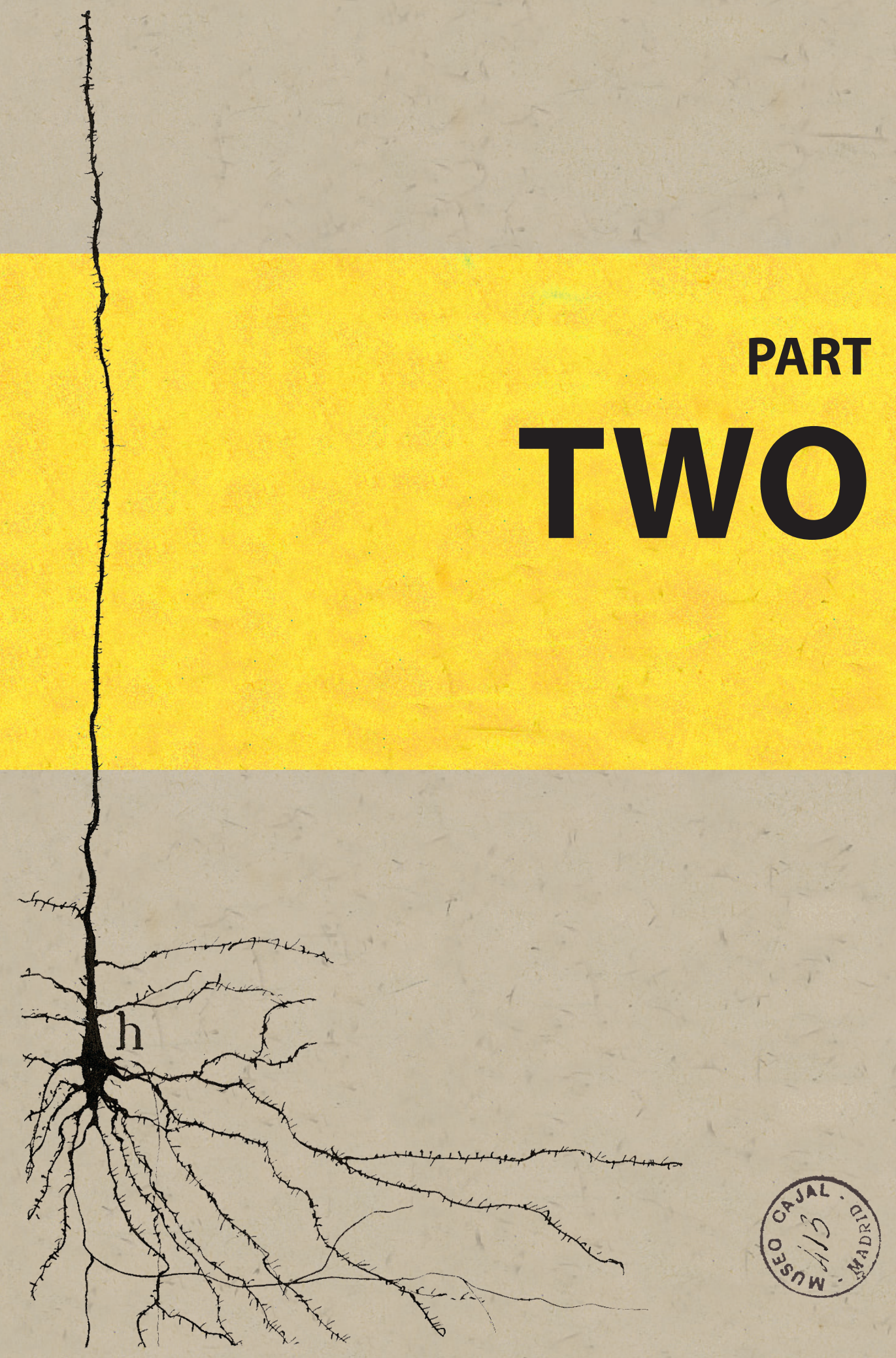




\section{AED WITHDRAWAL AFTER EPILEPSY SURGERY}




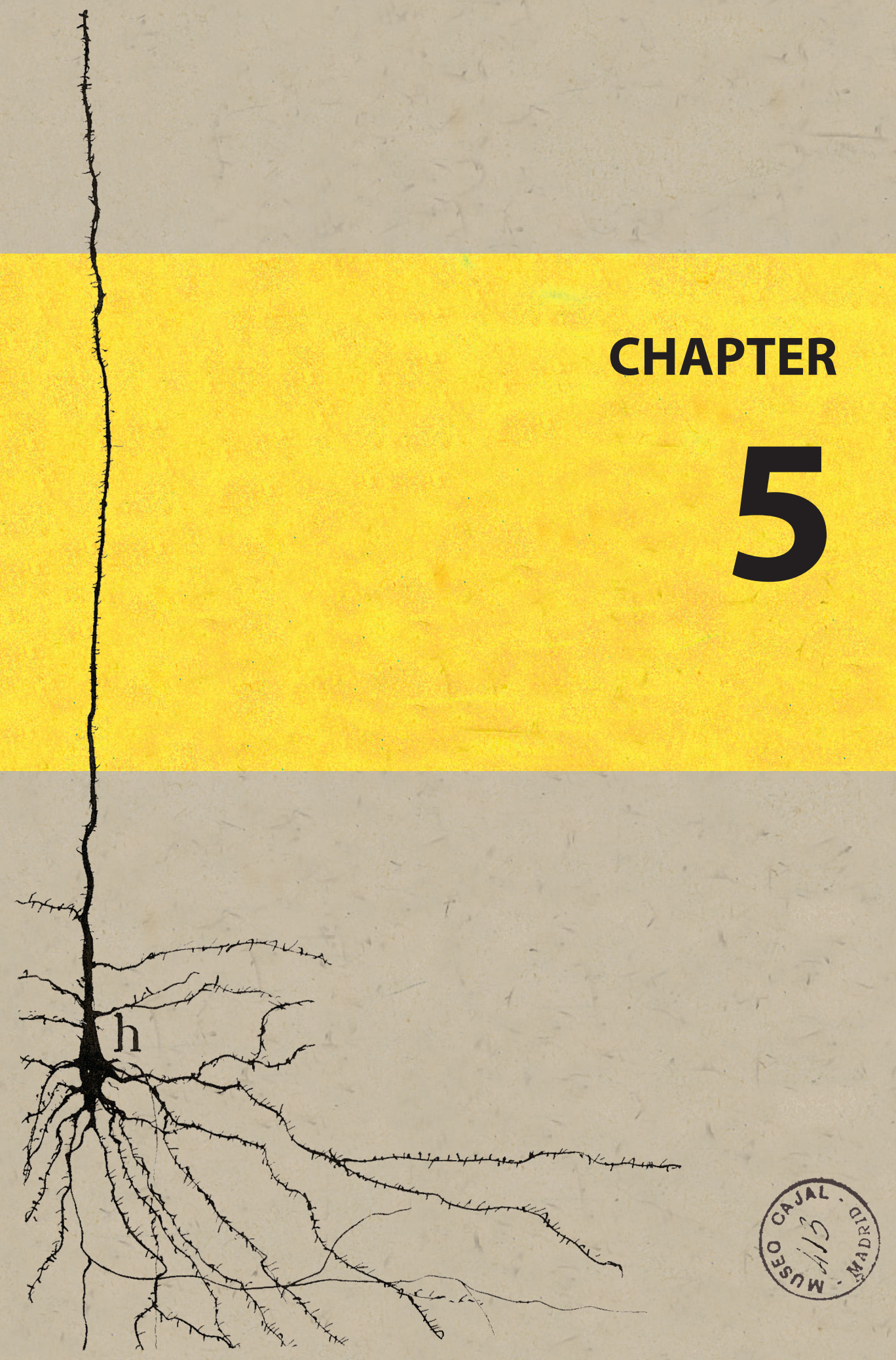




\section{Changing profiles of pediatric epilepsy surgery candidates over time: A nationwide single-center experience from 1990 to 2011}

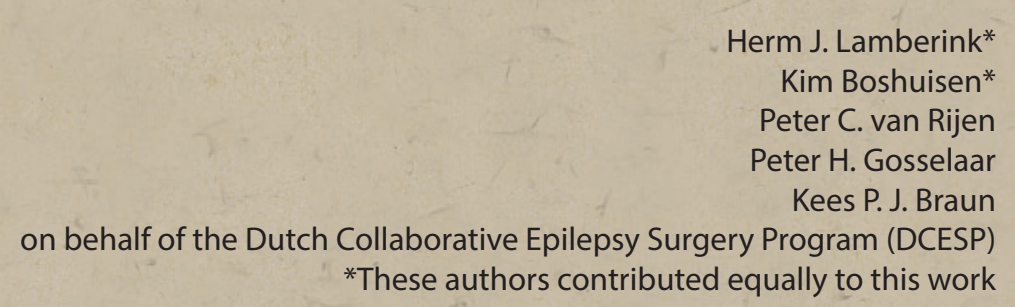

Epilepsia. 2015;56(5):717-25 


\section{Summary}

Objective: Over the past decades, the number of epilepsy surgeries in children has increased and indications for surgery have broadened. We studied the changes in patient characteristics between 1990 and 2011 in a nationwide cohort and related these to seizure outcome and postoperative medication status. Second, we tried to identify predictors for seizure outcome after pediatric epilepsy surgery.

Methods: To study changes over time, we divided this retrospective cohort of 234 children into two consecutive time periods of 11 years, and statistically compared the epochs in terms of patient characteristics, surgical variables, complications, seizure outcome, and postoperative medication status. To identify predictors of postoperative seizure freedom, we performed univariable and multivariable logistic regression analyses.

Results: The number of surgeries per year increased from an average of 5 in the first, to 16 in the past epoch. Over time, significantly more surgeries were performed for malformations of cortical development, and more patients underwent magnetoencephalography (MEG) and invasive monitoring. Four percent of patients had a serious complication. Complete seizure freedom (Engel class IA) at 2 years after surgery was achieved in $74 \%$ of patients, which did not change significantly over time. The proportion of patients who were free from seizures and antiepileptic medication 2 years after surgery significantly increased from $13 \%$ to $32 \%$. Factors predictive of seizure recurrence were preoperative intracranial monitoring, multilobar surgery, etiology, and longer duration of epilepsy before surgery.

Significance: Although more complex cases were operated over time and medication was withdrawn earlier after surgery, success rates at 2 years remained stable. In combination with low complication rates, this underscores the efficacy and safety of pediatric epilepsy surgery. It is important to consider epilepsy surgery early, as longer duration of epilepsy increased the risk of postoperative seizure recurrence. 
Epilepsy surgery has become a widely accepted treatment option for children with refractory epilepsy caused by a focal or hemispheric structural epileptogenic source. Between $59 \%$ and $70 \%$ of children become seizure-free after resective surgery (Spencer and Huh 2008). The ultimate goal of surgery is to "cure" the epilepsy, which can be defined as reaching complete freedom from both seizures and antiepileptic drugs (AEDs) for at least 5 years (Schmidt, Baumgartner, and Wolfgang 2004).

Over the past three decades, the approach to pediatric epilepsy surgery has changed dramatically. Improved imaging techniques with higher field strengths (Phal et al. 2008), specific magnetic resonance imaging (MRI) protocols, and imaging postprocessing in apparent MRI-negative patients have enabled the identification of patients previously not considered eligible for epilepsy surgery (So and Lee 2014). Other factors that have led to increased numbers of surgeries in children are advances in functional imaging (positron emission tomography/single-photon emission computed tomography [PET/SPECT]), improved source localization techniques, and intracranial monitoring (grid, stereo-electroencephalography [EEG]) (So and Lee 2014). The past ten years, attitudes toward surgery have changed even more, with the notion that surgery can also be performed in generalized epileptic syndromes when focal or hemispheric MRI abnormalities are present (Wyllie et al. 2007; Lachhwani 2005; Boshuisen et al. 2010), and the increasing awareness that early surgery (and thus shorter duration of epilepsy) leads to improved seizure and cognitive outcomes (Freitag and Tuxhorn 2005; Lettori et al. 2008).

Different studies have shown that the number of patients considered for epilepsy surgery has increased over the years (Bien et al. 2013; Schiltz et al. 2013), but the number of surgeries has remained stable (Schiltz et al. 2013; Englot et al. 2012). In children, however, the number of surgeries has increased with time (Schiltz et al. 2013; Hemb et al. 2010). Few studies have focused on trends in individual patient characteristics, and the studies that have done so were either in a specific population of patients who underwent temporal lobe epilepsy surgery (Helmstaedter et al. 2014), or were single-center studies and therefore carried the risk of presenting selected populations (Bien et al. 2013; Hemb et al. 2010). In only one study was pediatric epilepsy surgery addressed specifically (Hemb et al. 2010), whereas the others described populations including all ages (Bien et al. 2013; Helmstaedter et al. 2014).

Since 1990, children who are considered candidates for epilepsy surgery in The Netherlands are evaluated during the national multidisciplinary meetings of the Dutch Collaborative Epilepsy Surgery Program (DCESP). When consensus to perform surgery is reached, all children undergo surgery in the University Medical Center Utrecht (UMCU). The number of surgeries has increased rapidly over the past years. Because pediatric epilepsy surgery in our country has been performed in only a single center, this has created the unique opportunity to study characteristics and outcomes of a nationwide patient population over time. The current study provides an overview of pediatric epilepsy surgery in The Netherlands from 1990 to 2011.

Under the assumption that the rising numbers of pediatric epilepsy surgical procedures are paralleled by an increase in complexity of selected cases, we first aimed to explore how patient 
characteristics, surgical variables, and complication rates changed over two consecutive periods from 1990-2011. Second, we compared seizure outcome and medication status 2 years after surgery between these epochs. Finally, we identified predictive factors of seizure outcome in the entire surgical cohort.

\section{Methods}

Patients

This is a retrospective study of all children (aged $<18$ years) who underwent epilepsy surgery in UMCU and who had at least 2 years of follow-up. Patients were included if they underwent resective or disconnective surgery between January 1, 1990 and October 1, 2011. Excluded were patients who underwent palliative procedures (e.g., corpus callosotomy, $n=16$ ). For patients who were subject to a second surgical procedure $>2$ years later, only the first surgery was evaluated in the current analysis. In total, 234 patients were included.

\section{Data collection}

Data were retrieved from the database of the DCESP and complemented with information from patient records when necessary. Part of this cohort has been included in a previous publication on surgical outcome (Oijen et al. 2006). The DCESP receives referrals from all over the country. All results of diagnostic evaluation, partly performed in the referring center, are discussed in the monthly national multidisciplinary meetings, where it is decided whether additional investigations are necessary, and if the patient is eligible for surgery. Over the years, a total of three specialized epilepsy neurosurgeons performed all surgeries.

We collected data on gender, age at onset of epilepsy, age at surgery-including the proportion of children below the age of 2 years-duration of epilepsy, presence of specific electroclinical syndromes reflecting epileptic encephalopathy, presurgical evaluations, side and type of surgery, etiology of epilepsy, and number and type of complications. Seizure outcome was evaluated after 2 years, and expressed as the proportion of patients with Engel class IA (i.e., being completely seizure-free since surgery) (Engel et al. 1993). Furthermore, we collected data on antiepileptic drug (AED) use 2 years after surgery. Invasive monitoring included grids, depth electrodes, stereo-EEG recordings or a combination. Type of surgery was classified as "lobar," including frontal, temporal, parietal, and occipital resections or disconnections, "multilobar" resections or disconnections, and peri-insular hemispherectomy, from now "hemispherectomy." Electroclinical syndromes suggesting epileptic encephalopathy were defined according to the 2010 International League Against Epilepsy (ILAE) classification (Berg et al. 2010), including Ohtahara, West, Lennox-Gastaut syndromes, and electrical status epilepticus during sleep/continuous spikes and waves during sleep (ESES/CSWS). Etiology was classified according to histopathologic investigation (when available) or MRI. We distinguished "malformations of cortical development" (MCDs) (focal cortical dysplasia, hemimegalencephaly, and 
polymicrogyria), "tumors" (glioneuronal tumors and low-grade gliomas), "hippocampal sclerosis," "vascular pathology" (ischemic and hemorrhagic stroke, arteriovenous malformations), "neurocutaneous disorders" (Sturge-Weber syndrome and tuberous sclerosis complex), and "other" (including Rasmussen's encephalitis and unclassifiable pathology).

Complications were classified as either "serious" or "mild and reversible." Serious complications included perioperative death, complications that required invasive treatment, and unforeseen neurologic deficits that persisted for longer than 3 months. Hydrocephalus was reported when occurring at any moment postoperatively (not restricted to the first 3 months) and classified as a serious complication. Mild and reversible complications were classified into four subcategories: (1) surgery-related infections, divided into proven infections (with positive cultures), and suspected infections (but no culture performed) or aseptic inflammatory reactions (negative cultures); (2) non-surgery-related infections such as urinary tract, and upper or lower respiratory tract infections; (3) electrolyte changes for which intervention was started; and (4) transient neurologic deficits that were not anticipated. Foreseen neurologic deficits (i.e., homonymous hemianopia after hemispherectomy) were not considered as true complications.

\section{Study design and statistical analysis}

To study changes over time with regard to preoperative, perioperative, and postoperative variables, the cohort was divided into two epochs of 11 years: children who underwent surgery between 1990 and $2000(n=56)$, and between 2001 and $2011(n=178)$. The proportions of categorical variables were compared between the two epochs using X2-statistic, where necessary computed with Fisher's exact testing. For continuous variables, means were compared using a Student's t-test or, when data were not normally distributed, Mann-Whitney U-test.

To identify predictors of seizure freedom, first univariable logistic regression was performed for all variables included in this study. Variables with a $p$-value less than 0.10 were then included in a multivariable model. The separate lobar surgery locations were not included in the multivariable regression model because these are subgroups of lobar surgeries already included in the model.

Analyses were performed using IBM SPSS Statistics for Windows version 20.0, (IBM Corp. Armonk, NY, U.S.A.). 


\section{Results}

\section{Patient characteristics}

Between 1990 and 2011, 690 children were referred to the DCESP to discuss eligibility for epilepsy surgery (Table 1). Of these, 234 were operated and fulfilled the inclusion criteria. The proportion of evaluated patients receiving surgery was $47 \%$ between 1990 and 2000, and 48\% between 2000 and 2011 ( $p=0.94$ ). The number of surgeries increased by a factor of three over time; from an average of five surgeries per year in the first epoch, to an average of 16 in the last $(p<0.001)$. The median duration between epilepsy onset and surgery decreased with 2.1 years, from 5.8 years in the first epoch to 3.7 in the last, although this difference was not statistically significant $(p=0.07)$. Age, gender, and side of surgery did not differ between the two epochs. The contribution of the different etiologies changed significantly over time $(p=$ 0.03 ), with a relative increase of patients with malformation of cortical development and a decrease in "other pathologies." Nine percent of children had a clearly described electroclinical syndrome reflecting epileptic encephalopathy at time of surgery. A threefold increase in the proportion of these patients was seen over time, although not significantly.

\section{Preoperative additional investigations}

Supplementary to standard diagnostic examinations-including EEG and MRI-30\% of the patients needed additional diagnostic testing to further localize the epileptogenic source ( $\mathrm{Ta}$ ble 1). These included magnetoencephalography (MEG), PET, SPECT, or intracranial monitoring with subdural grids, depth electrodes, stereo-EEG, or a combination. MEG was used more frequently over time, from $4 \%$ to $16 \%(p=0.02)$, whereas the number of patients evaluated with SPECT has decreased from $13 \%$ to $3 \%(p=0.009)$. We did, however, perform SPECT registrations in the latter epoch and are using it more frequently in current practice, but in the presented cohort patients evaluated by SPECT have not been found eligible for surgery. The proportion of invasive recordings increased significantly from $2 \%$ to $12 \%$ of patients.

\section{Surgical procedures}

Twenty-seven percent of the surgeries were hemispherectomies, $6 \%$ were multilobar resections, and $68 \%$ lobar resections or disconnections (Table 1). Types of surgery did not change over time. Of the lobar surgeries, $71 \%$ were temporal. The number of frontal surgeries increased, although not significantly. 
Table 1 | Trends in time - general, pre-surgical, and surgical variables.

\begin{tabular}{|c|c|c|c|c|}
\hline Variable & $\begin{array}{l}\text { All years } \\
(n=234)\end{array}$ & $\begin{array}{l}1990-2000 \\
(n=56)\end{array}$ & $\begin{array}{l}2001-2011 \\
(n=178)\end{array}$ & p-value \\
\hline Evaluated patients in DCESP per year, mean (SD) & $31.4(19.6)$ & $16.9(8.9)$ & $45.8(16.3)$ & $<0.001$ \\
\hline Surgeries per year, mean (SD)* & $10.6(6.9)$ & $5.1(3.0)$ & $16.2(4.7)$ & $<0.001$ \\
\hline Gender female, $\%$ & 48.3 & 53.6 & 46.6 & 0.37 \\
\hline Age at seizure onset, $y$, median & 2.0 & 2.0 & 2.0 & 0.98 \\
\hline Age at surgery, $y$, median & 10.4 & 11.4 & 9.4 & 0.48 \\
\hline Age $<2$ years at surgery, $\%$ & 12.8 & 10.7 & 13.5 & 0.59 \\
\hline Duration of epilepsy until surgery, y, median & 4.2 & 5.8 & 3.7 & 0.07 \\
\hline Left side, \% & 48.7 & 42.9 & 50.6 & 0.31 \\
\hline Electroclinical syndrome ${ }^{* *}, \%$ & 8.6 & 3.6 & 10.1 & 0.17 \\
\hline West syndrome, \% & 6.0 & 1.8 & 7.3 & \\
\hline Ohtahara syndrome, $\%$ & 1.7 & 1.8 & 1.7 & \\
\hline ESES/CSWS, \% & 0.9 & 0 & 1.1 & \\
\hline Lennox-Gastaut syndrome, \% & 0.4 & 0 & 0.6 & \\
\hline Additional diagnostics***, \% & 29.5 & 23.2 & 31.5 & 0.24 \\
\hline PET, \% & 17.3 & 14.5 & 18.2 & 0.53 \\
\hline MEG, \% & 12.9 & 3.6 & 15.8 & 0.02 \\
\hline SPECT, \% & 5.2 & 12.7 & 2.8 & 0.009 \\
\hline Intracranial monitoring, \% & 9.4 & 1.8 & 11.8 & 0.03 \\
\hline Etiology & & & & 0.005 \\
\hline MCD, \% & 26.5 & 14.3 & 30.3 & 0.02 \\
\hline Tumor, \% & 34.6 & 37.5 & 33.7 & 0.60 \\
\hline Hippocampal sclerosis, \% & 14.1 & 19.6 & 12.4 & 0.17 \\
\hline Vascular, \% & 12.4 & 7.1 & 14.0 & 0.17 \\
\hline Neurocutaneous, \% & 7.7 & 8.9 & 7.3 & 0.69 \\
\hline Other, \% & 4.7 & 12.5 & 2.2 & 0.005 \\
\hline Surgery type & & & & 0.64 \\
\hline Hemispherectomy, \% & 26.9 & 23.2 & 28.1 & \\
\hline Multilobar, \% & 5.6 & 7.1 & 5.1 & \\
\hline Lobar, \% & 67.5 & 69.6 & 66.9 & \\
\hline Lobar surgery location & & & & 0.46 \\
\hline Temporal, \% & 70.9 & 74.4 & 69.7 & \\
\hline Frontal, \% & 21.5 & 15.4 & 23.5 & \\
\hline Parietal, \% & 5.1 & 5.1 & 5.0 & \\
\hline Occipital, \% & 2.5 & 5.1 & 1.7 & \\
\hline
\end{tabular}

Changes over time compared between two epochs. Bold p-values depict a statistically significant difference between the epochs. Analysis performed with independent samples t-test, Mann-Whitney U test, chi-square or Fisher's exact test when appropriate. ${ }^{\text {a }}$ Computed with 234 patients included in this study. ${ }^{\text {b }}$ Percentage of patients with one of four categories below. One patient was included with West syndrome and subsequent Lennox-Gastaut syndrome. An additional $2 \%$ of patients had a history of West syndrome (not in table), which resolved into focal seizures; these patients underwent surgery 3 to 12 years later. ' Percentage of patients who underwent one or more of the four diagnostic tests below. One patient can be included in more than one of the categories. DCESP, Dutch Collaborative Epilepsy Surgery Program; ESES, electrical status epilepticus during sleep; CSWS, continuous spikes and waves during sleep; PET, positron emission tomography; MEG, magnetoencephalography; SPECT, single photon emission computed tomography; MCD, malformation of cortical development. 


\section{Complications}

Four percent of all patients had a serious complication (Table 2). The proportion of serious and mild or reversible complications remained stable over the years $(p=1.00$ and $p=0.71$, respectively). From the patients with a serious complication directly related to surgery, only one $(0.4 \%)$ developed a permanent hemiplegia as result of an ischemic lesion after an insular resection. Eight patients (3.5\%) had to undergo invasive treatment for several complications, including intracranial hematoma, increased intracranial pressure, cerebrospinal fluid (CSF) leakage, and hydrocephalus. No deaths occurred as a complication of surgery. Within the follow-up period, two patients died: one of pulmonary failure during intensive care treatment for refractory epilepsy 3 months after surgery, and one at 6 months after surgery of unknown cause.

Mild complications included mainly infections or suspected infections (9.9\%). From the group of surgery-related possible infections, a positive CSF culture was identified in only $0.4 \%$. The remaining surgery-related presumed infections (4.3\%) where treated with antibiotics even despite negative cultures. The non-surgery-related infections (4.7\%) consisted of respiratory tract infections, urinary tract infections and gastroenteritis. Two percent of the patients had unforeseen neurologic deficits, which normalized within the first 3 months after surgery, and $3.5 \%$ of the patients received treatment for electrolyte disturbances after surgery.

\section{Surgical outcome}

Seizure outcome remained stable over the two epochs $(p=0.09)$, with $81 \%$ of the patients being free from disabling seizures (Engel class 116) after 2 years, and 74\% with complete seizure freedom since surgery (Engel class IA) (Table 3). Eight percent had rare disabling seizures (Engel class II). In total, approximately $89 \%$ of the patients had a clear benefit from surgery (Engel class I and II), 6\% had worthwhile improvement (Engel class III), and 6\% did not have any improvement (Engel class IV). The proportion of patients for whom AEDs were completely withdrawn within 2 years after surgery has significantly increased, from $14 \%$ to $33 \%$ ( $p=$ 0.006). Of all patients who were off antiepileptic drugs, $94 \%$ had been completely seizure-free since surgery. 
Table 2 | Trends in time - complications.

\begin{tabular}{|c|c|c|c|c|}
\hline Variable & $\begin{array}{l}\text { All years } \\
(n=230)\end{array}$ & $\begin{array}{l}1990-2000 \\
(n=55)\end{array}$ & $\begin{array}{l}2001-2011 \\
(n=175)\end{array}$ & p-value \\
\hline Serious complications, $\%$ & 3.9 & 3.6 & 4.0 & 1.00 \\
\hline Invasive treatment, $\%$ & 3.5 & 3.6 & 3.3 & \\
\hline Hydrocephalus, \% & 1.3 & 1.8 & 1.1 & \\
\hline Permanent neurological deficit, \% & 0.4 & 0 & 0.6 & \\
\hline Death, \% & 0 & 0 & 0 & \\
\hline Mild and reversible complications, $\%$ & 14.8 & 16.4 & 14.3 & 0.71 \\
\hline Infections, surgery related, $\%$ & 4.7 & 5.4 & 4.6 & \\
\hline Documented, \% & 0.4 & 1.8 & 0 & \\
\hline Suspected/aseptic, \% & 4.3 & 3.6 & 4.6 & \\
\hline Infections, non-surgery related, $\%$ & 5.2 & 7.3 & 4.6 & \\
\hline Transient neurological deficits, \%* & 1.7 & 1.8 & 1.7 & \\
\hline Intervention for electrolyte change, $\%$ & 3.5 & 1.8 & 4.0 & \\
\hline
\end{tabular}

Table 3 | Trends in time - surgical outcome.

\begin{tabular}{lllll}
\hline Variable & $\begin{array}{l}\text { All years } \\
(\mathbf{n = 2 3 4 )}\end{array}$ & $\begin{array}{l}\mathbf{1 9 9 0 - 2 0 0 0} \\
(\mathbf{n}=\mathbf{5 6})\end{array}$ & $\begin{array}{l}\mathbf{2 0 0 1 - 2 0 1 1} \\
(\mathbf{n = 1 7 8})\end{array}$ & p-value \\
\hline Completely seizure-free (SF), 2y, \% & 74.4 & 71.4 & 75.3 & 0.57 \\
Engel classification, 2y, \% & & & & 0.09 \\
1 & 81.2 & 78.6 & 82.0 & \\
2 & 7.7 & 5.4 & 8.4 & \\
3 & 5.6 & 12.5 & 3.4 & \\
4 & 5.6 & 3.6 & 6.2 & \\
AED-free, 2y, \% & 28.8 & 14.3 & 33.3 & 0.006 \\
SF and AED-free, 2y, \% & 27.0 & 12.5 & 31.6 & 0.005 \\
\hline
\end{tabular}

Trends in time in seizure outcome and medication status at 2 years after surgery. Bold $p$-values depict a statistically significant difference between the epochs. Analysis was performed with $\mathrm{x} 2$ or Fisher's exact test when appropriate.

SF, complete seizure freedom at 24 months (Engel class IA); AED, antiepileptic drug. 
In patients with a follow-up of at least 5 years $(n=136$, median follow-up 8 years), the proportion of patients with an Engel class I or IA score at latest follow-up was $75 \%$ and $65 \%$, respectively (Supplementary Table 1). The proportion of seizure- and AED-free patients was 55\%. No significant changes over time were observed. The median follow-up duration of patients operated during the first time epoch, however, was significantly longer than of those from the second epoch $(p<0.001)$. We examined possible relations between follow-up duration and the three long-term outcome measures using logistic regression. There was no significant relation between follow-up duration in years and Engel class I (odds ratio [OR] 0.98), Engel class IA (OR 1.00), or seizure and AED freedom (OR 1.05), at latest follow-up ( $p=0.76, p=0.97$, or $p=0.39$, respectively).

\section{Predictors of outcome}

We analyzed which factors were associated with complete seizure freedom (Engel class IA) (Table 4). In multivariable analysis, multilobar surgery (OR 0.18, $\mathrm{p}=0.02)$, presurgical invasive monitoring (OR $0.28, p=0.02$ ), and longer duration of epilepsy until surgery (OR 0.90 per year, $p=0.03$ ) were independently associated with unfavorable seizure outcome (not reaching Engel class IA). Etiology was also related with outcome, with "other etiology" (OR 0.18, p = 0.02) being an independent predictor of seizure recurrence.

\section{Discussion}

In this Dutch pediatric epilepsy surgery cohort, operated between 1990 and 2011, we studied patient and surgery characteristics to identify changes over time. We found several shifts in patient characteristics. The unselected and nationwide nature of this cohort provides a unique opportunity to study the evolving field of pediatric epilepsy surgery. Over the past two decades, the number of evaluated children for surgery and the number of surgeries increased significantly, with more operations on MCDs, and an increased proportion of preoperative MEG recordings and invasive monitoring. The complication rate was low and stable over time. Seizure outcomes at 2 years remained stable, with $90 \%$ of patients having achieved improvement in epilepsy severity and $74 \%$ being completely seizure-free since surgery. Over time, the interval to start of AED withdrawal shortened, resulting in significantly increased AED freedom at 2 years after surgery. Multilobar surgery, presurgical invasive monitoring, etiology, and longer duration of epilepsy independently predicted poor seizure outcome. 


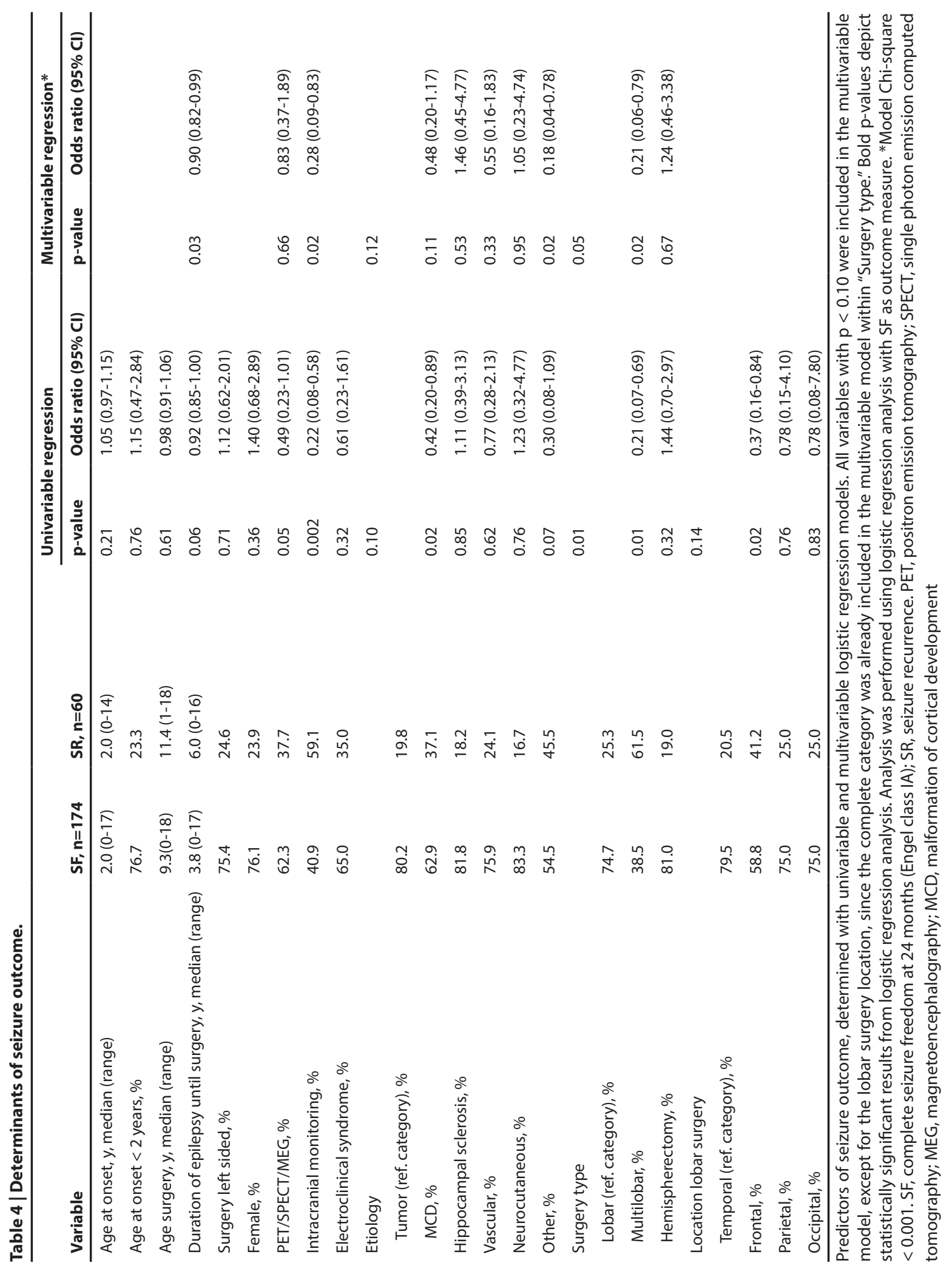




\section{Patient characteristics}

The relative increase in the number of patients with MCD, presence of an electroclinical syndrome of epileptic encephalopathy, and the increased proportion of patients with preoperative invasive monitoring, reflect a shift toward more complex cases. In a population of all ages, a similar trend toward more complex cases was observed with less resections on benign tumors and more on patients with focal cortical dysplasia (Bien et al. 2013). In our cohort, the number of frontal surgeries increased, although not significantly.

With regard to etiology, postoperative seizure freedom rates of children with malformations of cortical development, particularly those with focal cortical dysplasia (FCD) type I (Krsek et al. 2009), are known to be lower than those of patients with other pathologies. Chern et al. (Chern et al. 2010) reported a seizure freedom rate of $60 \%$ (range $46-70 \%$ ) for patients with FCD, compared to $71 \%$ (range 36-100\%) for tumor patients (Englot et al. 2011). In the current study, this lower seizure freedom rate for MCD patients was also found, with $63 \%$ of the patients reaching complete seizure freedom compared to success rates over $80 \%$ for tumor, hippocampal sclerosis, and vascular etiology. The changes in resection type and identified etiologies indicate a clear shift in the utilization of pediatric epilepsy surgery. At the time when many pediatric epilepsy surgery programs started, mainly children with well-circumscribed focal, MRI-visible, and preferably temporal structural lesions were considered eligible candidates for surgical treatment. Over the years, indications for surgery have now broadened, including children with frontal or posterior quadrant epilepsies, generalized epileptic encephalopathies with focal or hemispheric structural pathology (Wyllie et al. 2007; Lachhwani 2005; Boshuisen et al. 2010), and MRI-negative cases (Phal et al. 2008; So and Lee 2014; Bien et al. 2013).

The trends in the number of specific additional presurgical investigations support the hypothesis that more complex cases are operated in the latest years: the increased use of MEG and invasive monitoring are indicative of the need for more precise localization of the epileptogenic source. In our nationwide single-center experience, the availability of acute corticography since 2000, of 3T MRI since 2004, and of neuronavigation since 2003, improved diagnostic accuracy and the ability to delineate the epileptogenic zone. Despite the trend toward the selection of more complex cases, with inherent lower a priori probability of reaching seizure control, seizure outcomes were shown to remain remarkably constant over time, with around $75 \%$ of complete seizure freedom at 2 years. This likely reflects the team's learning curve, experience, and access to diagnostic procedures.

\section{AED withdrawal policy}

AED freedom rates in our cohort increased from $14 \%$ to $33 \%$, reflecting a more aggressive AED withdrawal policy, which seems to be a more global phenomenon; in a survey regarding AED withdrawal policy of $2007,71 \%$ of U.S. epileptologists would wait for at least 2 years of postoperative seizure freedom before considering discontinuation of drugs, and some of these would even never consider withdrawal of AEDs (Berg et al. 2007). In 2012 (Téllez-zenteno et al. 2012) 
and 2013 (Swisher and Sinha 2013), only 3-7\% of U.S. and Canadian epileptologists would wait to withdraw AEDs for at least 2 years in patients receiving polytherapy and $24-35 \%$ would wait 2 years for patients receiving monotherapy, reflecting a shift in postoperative drug withdrawal policy.

The proportion of patients who are ultimately AED free is different between children and adults, as shown in a Swedish study on long-term postoperative outcomes: 10 years after surgery, $86 \%$ of the children had discontinued AEDs, compared to $43 \%$ of adults (Edelvik et al. 2013). The timing of AED withdrawal, however, seems to be similar in children and adults: a meta-analysis including both adults and children reported an average time between surgery and initiation of AED withdrawal of 14 months, and the average time to complete discontinuation of 30 months (Ladino, Hernández-Ronquillo, and Téllez-Zenteno 2014). The TimeToStop study on 766 children revealed that in children the median time to start reducing drugs was 12.5 months, and the median time to discontinuation was 28.8 months (Boshuisen et al. 2012). In that study, early AED withdrawal was thought to unmask surgical failure sooner, but not at the cost of long-term outcomes. This was recently confirmed in a mainly adult cohort study (Yardi et al. 2014). The changes in survey responses point toward a trend of earlier AED withdrawal, similar to what we observed in the current study. In contrast to these findings, a study from the pediatric UCLA cohort (Hemb et al. 2010) attributed an increased seizure freedom

rate in recently operated children to longer AED use after surgery, as compared to children who underwent surgery in earlier time epochs.

\section{Predictors of outcome}

Longer duration of epilepsy, multilobar surgery, etiology, and intracranial presurgical monitoring were found to be independently associated with unfavorable seizure outcome. Other known factors predicting seizure recurrence in pediatric epilepsy surgery include extratemporal resections, multifocal MRI lesions, incomplete resection of the lesion, and the presence of presurgical generalized tonic-clonic seizures (Spencer and Huh 2008).

Intracranial monitoring has previously been reported to predict unfavorable postoperative seizure outcome (Tonini et al. 2004). The predictive value of intracranial monitoring, however, may highly depend on the population, since other studies have reported no or an inverse relation between invasive monitoring and seizure outcomes (Kumar et al. 2013; Brelie et al. 2013; Elsharkawy et al. 2008).

Longer duration of epilepsy until surgery was associated with unfavorable seizure outcome, confirming findings from previous studies (Englot et al. 2011; Edelvik et al. 2013; Simasathien et al. 2013; Englot and Chang 2014). In addition, it has been demonstrated previously that longer duration of epilepsy has a negative impact on cognitive change after surgery (Spencer and Huh 2008; Van Schooneveld and Braun 2013). We may expect that the recent evidence on the beneficial cognitive effects of early epilepsy surgery lead to a further decrease in age at surgery in future pediatric cohorts. 


\section{Complications}

The risk of serious surgical complications was $4 \%$, with only $0.4 \%$ resulting in permanent neurologic damage. The frequency of mild complications was higher, but it should be acknowledged that not all presumed surgical complications are true complications requiring therapy. In some children, antibiotic treatment was started when infection was suspected, but only in a minority was direct proof of infection by positive culture obtained. After pediatric hemispherectomy, for example, fever, increased peripheral white blood cell count, and CSF protein are frequently observed, but this is rarely caused by intracranial infection (Phung, Mathern, and Krogstad 2013). Changes in serum sodium levels in some children led to treatment (fluid restriction or sodium supplementation) without direct proof of syndrome of inappropriate antidiuretic hormone secretion (SIADH) or cerebral salt wasting; the electrolyte disbalance may have also been caused by specific AEDs (e.g., carbamazepine). If we do not take above-mentioned "mild complications" into account, only $2.1 \%$ of the patients had a mild surgery-related complication (documented infections and transient neurological deficits).

Several studies have used different criteria to report their complication rates. A recent review on complications after epilepsy surgery reported $5.7 \%$ minor and $4.5 \%$ major medical complications, and $11.2 \%$ minor and $5.1 \%$ major neurologic complications for children (Hader et al. 2013). The higher complication rate in that study can be explained by the authors' choice to include anticipated neurologic deficits that are inherent to surgery. Hemb et al. (Hemb et al. 2010) have reported serious and permanent complications only, with a complication rate of $9.2 \%$. The difference in complication rate between their and our cohort can be explained by the much higher percentage of hemispherectomy patients in their cohort and the difference in definition of complications that included long-term deaths unrelated to surgery in the cohort of Hemb et al., whereas we chose not to consider those cases.

The 22 children who underwent invasive EEG monitoring preoperatively did not have any complication related to this procedure. Studies in larger cohorts have concluded that invasive monitoring bears limited risks, and large benefits (Hedegärd et al. 2014; Wellmer et al. 2012), with a risk for major or permanent complications of $0.6 \%$ (Hader et al. 2013).

\section{Strengths and limitations}

The current study distinguishes itself from previous studies because of its nationwide setup. The population included all children who underwent epilepsy surgery in The Netherlands between 1990 and 2011, and therefore the observed trends are truly reflective of changes in pediatric epilepsy surgery in an unbiased cohort, although we acknowledge that there will be differences between countries and continents.

The study also has several limitations. First, no data are presented on changes in preoperative evaluations and diagnostics for children who were referred but eventually rejected for epilepsy surgery. There was an increasing number of patients referred to the DCESP, which reflects the increased knowledge of the benefits of epilepsy surgery among physicians taking care of 
children with refractory epilepsy.

Second, the follow-up period of 2 years after surgery is relatively short. When our success rate is compared to other cohorts, the proportion of completely seizure-free patients of $74 \% 2$ years after surgery is high, since the average postoperative seizure freedom rate in children is reported to be $59-70 \%$ (Spencer and Huh 2008). However, seizure-freedom rates are known to decline over the years (Tisi et al. 2011) and seem to remain stable after 5 years postoperatively (Edelvik et al. 2013). For 136 of our patients, follow-up data after at least 5 years was available, revealing a relatively high proportion of patients with Engel class IA (65\%), with no differences between the two epochs. However, a direct comparison of long-term outcome between the two time periods is complicated by the inherent differences in follow-up duration.

\section{Conclusion}

The outcome of pediatric epilepsy surgery in The Netherlands has been stable over time with high success rates and low complication rates. Although we found a shift toward surgery in more complex patient categories (such as MCDs), and a tendency toward more complex lesion locations (such as frontal resections), seizure-freedom rates remained steadily high, and the number of children who reached both freedom from seizures and AEDs significantly increased over the years. Predictors independently associated with unfavorable seizure outcome were multilobar surgery, invasive monitoring, and longer epilepsy duration. Unfavorable seizure outcomes with longer duration of epilepsy underscore the fact that early surgery should be the goal. The high seizure freedom and low complication rates in this nationwide cohort reflect the advantage of a centralized multidisciplinary assessment of surgical candidates, and the performance of surgery in highly specialized medical centers.

\section{Acknowledgments}

We gratefully acknowledge Dr. C.S.P. Uiterwaal and Dr. F.S.S. Leijten for their valuable comments. 
Supplemental table 1 | Long term follow-up.

\begin{tabular}{lllll}
\hline Variable & $\begin{array}{l}\text { All years } \\
(\mathbf{n = 1 3 6 )}\end{array}$ & $\begin{array}{l}\mathbf{1 9 9 0 - 2 0 0 0} \\
(\mathbf{n = 5 5 )}\end{array}$ & $\begin{array}{l}\mathbf{2 0 0 1 - 2 0 1 1} \\
(\mathbf{n = 8 1 )}\end{array}$ & p-value \\
\hline FU duration in years, median (range) & $8(5-17)$ & $11(5-17)$ & $7(5-12)$ & $<\mathbf{0 . 0 0 1}$ \\
Engel 1, \% & 75.0 & 76.4 & 74.1 & 0.76 \\
Engel 1A, \% & 65.4 & 65.5 & 65.4 & 1.00 \\
SF and AED free, \% & 55.2 & 63.0 & 50.0 & 0.14 \\
\hline
\end{tabular}

Long-term seizure and medication outcome, in patients with follow-up of at least five years. Analysis was performed with Chi-square or Mann-Whitney-U tests. 


\section{APPENDIX}

Dutch Collaborative Epilepsy Surgery Program

Current and former* members:

University Medical Center Utrecht

K.P.J. Braun, F.E. Jansen, K. Geleijns, O van Nieuwenhuizen* (pediatric neurologists); F.S.S. Leijten, C.H. Ferrier, A.C. van Huffelen* (clinical neurophysiologists); P.C. van Rijen, P. Gosselaar, C.W. van Veelen* (neurosurgeons); G.A. de Kort (neuroradiologist); M.M. van Schooneveld, O. Braams, M.J. van Zandvoort (neuropsychologists); W.T. Spliet (neuropathologist); T.A. Gebbink (physician assistant); J.M. Ophorst-van Eck (specialized nurse); T. De Groot (secretary).

Epilepsy Centers of the Netherlands Foundation (SEIN)

R.M. Debets* (neurologist, adult epileptologist); D. Velis, S. Claus, M. Bourez-Swart, A.W. de Weerd, W. van Emde Boas* (clinical neurophysiologists, epileptologists); W.C. Alpherts* (neuropsychologist); A. Hospes (specialized nurse).

Epilepsy Center Kempenhaeghe

A.J. Colon, L. Wagner, H.W. ter Spill*, E.P. Veltman* (clinical neurophysiologists, epileptologists). Free University Medical Center Amsterdam

J.C. Baayen (neurosurgeon); C.J. Stam, H.E. Ronner (clinical neurophysiologists); E. Comans (nuclear radiologist); E. Sanchez (neuroradiologist).

Maastricht University Medical Center

J. Dings (neurosurgeon); V. van Kranen-Mastenbroek (clinical neurophysiologist); P. Hofman (neuroradiologist). 


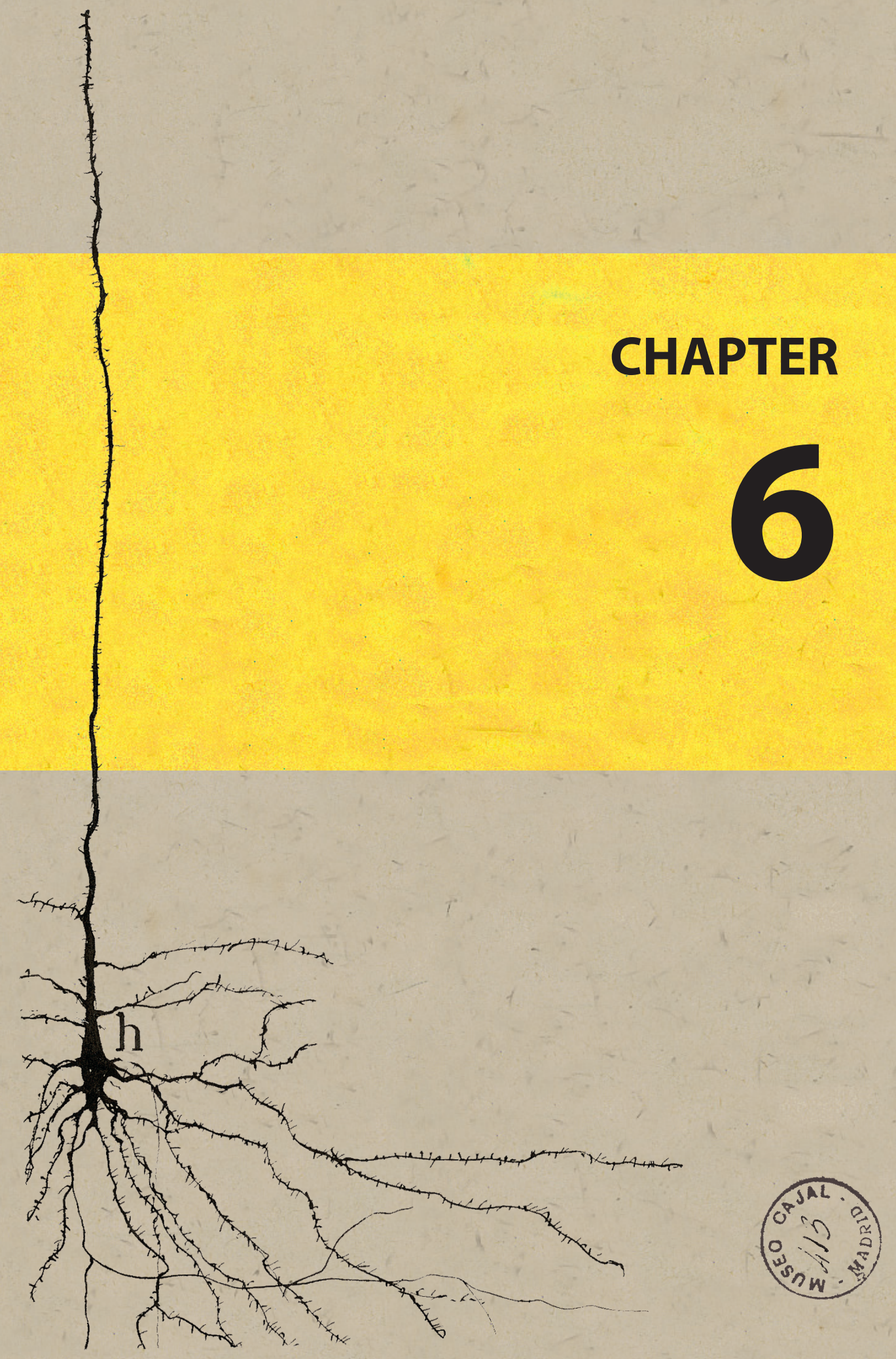




\section{Aetiology and duration of epilepsy matter - results from a multicentre European histopathology study of 9,147 patients who underwent epilepsy surgery}

Herm J Lamberink Willem M Otte Karin Geleijns

Eleonora Aronica

Roland Coras

Hajo Hamer

Karl Rössler

Christian G Bien

Thomas Cloppenborg

Alexander Grote

Johannes Schramm

Albert Becker

Christian Elger

John S Duncan

Jane De Tisi

Maria Thom

Francine Chassoux

Bertrand Devaux

Georg Dorfmüller

Mathilde Chipaux

Kristina Malmgren

Bertil Rydenhag

Andreas Schulze-Bonhage

Jürgen Beck

Thomas S Jacques

J Helen Cross

Cigdem Özkara

Mustafa Uzan

Tom Pieper

Manfred Kudernatsch
Giancarlo DiGennaro

Vincenze Esposito

Sylvain Rheims

Helene Catenoix

Istvan Bodi

Mrinalini Honavar

Petr Marusic

Adam Kalina

Reetta Kälviäinen

Leena Jutila

Margitta Seeck

Karl Lothar Schaller

Soheyl Noachtar

Katharina Ernst

Thomas Grunwald

Kristina Koenig

Wim Van Paesschen

Johannes van Loon

Tom Theys

Raf Sciot

Armen Melikyan

Lyudmila Shishkina

Martha Feucht

Theresa Scholl

Jose Pimentel

Isabel Amorim

Tim J von Oertzen

Martin Aichholzer

Rita Garbelli

Francesco Deleo
Felix Rosenow

Katja Menzler

Karl Martin Klein

Pavel Krsek

Martin Kudr

Olaf Schijns

Jan Beckervordersandforth

Victoria San Antonio-Arce

Javier Aparicio

Alexis Arzimanoglou

Joseph Toulouse

Renzo Guerrini

Carmen Barba

Antonia Jakovcevic

Zeljka Petelin Gadze

Nicola Specchio

Luca De Palma

Savo Raicevic

Dragoslav Sokic

Joana Ribeiro

Francisco Sales

Antonio Gil-Nagel

Rafael Toledano Delgado

Ingmar Blümcke*

Kees PJ Braun*

on behalf of the European

Epilepsy Brain Bank consortium

*authors contributed equally 


\section{Abstract}

Background: Epilepsy surgery is a widely accepted treatment option for drug-resistant focal epilepsy. A detailed analysis of longitudinal postoperative seizure outcomes and use of antiepileptic drugs across different brain lesions causing epilepsy is not available. Such information would improve presurgical decision-making and counselling.

Methods: Patients who had epilepsy surgery between 2000 and 2012 at 37 collaborating centres of the European Epilepsy Brain Bank consortium were studied. Histopathological diagnosis and a minimal dataset of clinical variables were collected. Outcome data included seizureand medication-freedom at one, two, and five years. Proportions of seizure- and medicationfree subjects were reported for the seven main categories of histopathological diagnosis. We studied patterns of seizure and medication-freedom over time. Relative outcomes were computed using random effects multivariable logistic regression and the association between histopathology, duration of epilepsy, age at surgery and outcomes was characterised.

Findings: 9,147 patients were included. The diagnoses tumour, vascular malformation and hippocampal sclerosis had the best seizure outcome, with 76\% (1239/1635), 74\% (328/443), and $72 \%(2108 / 2948)$ of subjects being seizure-free at two years after surgery, respectively. Lower rates of seizure-freedom were seen for malformation of cortical development $60 \%$ (1229/2040), encephalitis 60\% (74/124), glial scar 59\% (155/261), and those with no histopathological lesion $54 \%$ (396/740). The proportion of both seizure- and medication-free patients was 3-9\% at one year and increased to $17-43 \%$ at five years. Children were more often seizure- and drug-free, temporal lobe surgeries had the best seizure outcomes, and a longer duration of epilepsy was associated with reduced chance of seizure-freedom and seizure- and drug-freedom.

Interpretation: This large, multi-centre cohort offers precise estimation of seizure- and drugfree outcomes up to five years after resective brain surgery for epilepsy. Two-thirds of patients remained seizure-free. Histopathological diagnosis, age at surgery and duration of epilepsy are important prognostic factors. 


\section{Research in context}

Evidence before this study. We searched PubMed for original cohort studies describing outcomes of epilepsy surgery related to histopathology or aetiology, using the terms "epilepsy surgery", "outcome OR seizure", and "histopathology OR histopathological OR etiology OR aetiology", without language restriction. Cohorts focusing on specific pathologies or brain regions were excluded since direct comparison of outcomes between pathologies would not be possible, as well as studies without a comparison in surgery outcomes between different aetiologies. The search yielded 252 results. Only five identified studies had sample sizes above five hundred patients. The largest cohort sample was 1,995. None of these studies included all pathological categories, including encephalitis and vascular malformations. Only one study included a category consistent with a brain scar. The only aetiology included in all five studies was hippocampal sclerosis, four looked at either focal cortical dysplasia or the total group of malformations of cortical development, and only three looked at outcomes for tumours. The identified studies did not provide detailed information on expected surgical outcomes for all classes of histopathological diagnoses, nor for specific diagnoses. Postoperative drug-freedom was not reported in any of the studies. None studied the effect of duration of epilepsy in the separate diagnoses.

Added value of this study. This study provides clear-cut estimates of epilepsy surgery outcomes up to five years after surgery, for all major classes of histopathological diagnosis and specific sub-diagnoses, based on 9,147 operated patients. There are important differences between the aetiologies regarding seizure- and drug-freedom, changes in seizure-freedom over the postoperative years, and the effect of duration of epilepsy on surgical outcome.

Implications of all the available evidence. Epilepsy surgery should be considered in every person with drug-resistant focal epilepsy: surgery renders the majority of selected patients seizure-free. Histopathology is an important predictor of seizure outcome. Other independent determinants of seizure- and drug-outcome are age at surgery, cerebral lobe, and duration of epilepsy. Longer duration of epilepsy is associated with poorer outcome. 


\section{Introduction}

Epilepsy is one of the most prevalent and severe neurological disorders with around 70 million individuals affected worldwide (Ngugi et al. 2010). Antiepileptic drugs are effective in approximately 70\% of patients (Kwan and Brodie 2000; Picot et al. 2008), may have many side effects (Perucca et al. 2009), and merely suppress seizures, rather than modifying the disease course. Approximately $60 \%$ of drug-resistant patients with focal epilepsy have been reported to become seizure free one year after surgery (Blümcke et al. 2017) and elective surgery is increasingly recognized as curative treatment option (Ryvlin, Cross, and Rheims 2014). The range of reported seizure-freedom remains large, however, varying from 15\% to 93\%, depending among other factors - on the number of seizures occurring before surgery, MRI findings, need for invasive diagnostics, definition of seizure freedom and duration of post-surgical follow-up (Ryvlin, Cross, and Rheims 2014; Gracia et al. 2015; Jobst and Cascino 2019; Bell et al. 2017). The underlying brain pathology is likely to also play a role in outcome assessment of surgical treatment (Jehi et al., 2015). Studying postoperative seizure-freedom rates in relation to the large spectrum of brain lesions and duration of follow-up, while adjusting for many different determinants of outcome, is a particular challenge and requires a large cohort of surgical patients and a multi-center approach.

We recently described the spectrum of histopathological diagnoses in 9523 European patients who underwent epilepsy surgery between 1990 and 2014 (Blümcke et al. 2017). The study confirmed the unbalanced distribution of various disease conditions, and also showed that the proportion of histopathological categories varied largely between children and adults (Blümcke et al. 2017).

In the current study, we aimed to substantiate (1) the association between histopathology and seizure outcome and drug-freedom up to five years after epilepsy surgery, and (2) the effects of age at surgery and duration of epilepsy among different histopathological diagnoses on outcome, in a cohort of 9147 epilepsy surgery patients across Europe.

\section{Methods}

\section{Study design and participants}

The European Epilepsy Brain Bank is an EU-funded open collaboration of specialised epilepsy centres in Austria, Belgium, Croatia, the Czech Republic, Finland, France, Germany, Greece, Italy, the Netherlands, Portugal, the Russian Federation, Serbia, Spain, Sweden, Switzerland, Turkey, and the United Kingdom. Histopathological diagnoses of resected brain tissue were made at the local centres or the German Neuropathology Reference Center for Epilepsy Surgery in Erlangen, Germany (Blümcke et al. 2017). Patients in this database were included in the current study if epilepsy surgery was performed between January 2000 and December 2012. Information 
regarding basic clinical parameters was provided by the participating centres. Collected data included sex, age at surgery (children, aged 0-17 years, versus adults), age at onset of epilepsy, duration of epilepsy prior to surgery, lobe of surgery (temporal, frontal, occipital, parietal or multilobar [including hemispheric], and hypothalamic resections), and side of surgery (left, right, and midline, i.e. hypothalamus). Participating centres were asked to provide seizure outcomes (classified using either Engel or ILAE scores) and antiepileptic drug (AED) use at 1, 2 and 5 years. Patients were included in the study when at least seizure outcome was available for one of these time-points. In case of multiple surgeries, only histopathology and outcome data related to the first surgery were included. Histopathological diagnoses were divided into seven main categories: (1) no lesion (normal tissue or non-specific gliosis); (2) glial scarring (as result of an acute vascular event, i.e. hypoxemic or haemorrhagic infarction, trauma, or infection in the past); (3) encephalitis (Rasmussen encephalitis and 'other'); (4) malformation of cortical development (MCD) (focal cortical dysplasia [FCD] type I, type II and FCD not otherwise specified, mild MCD, hypothalamic hamartoma, tuber, and'other'rare conditions [including glioneuronal hamartoma, hemimegalencephaly, heterotopia, polymicrogyria]); (5) hippocampal sclerosis; (6) vascular malformation (cavernoma or 'other' [including meningioangiomatosis, as seen in Sturge-Weber syndrome, and arterio-venous malformations]), and (7) low-grade epilepsy-associated tumour (LEAT; dysembryoplastic neuroepithelial tumour, other lowgrade neuroepithelial tumours, diffuse glioma, ganglioglioma, pilocytic astrocytoma, and 'other'). The latter category included angiocentric neuroepithelial tumor, cyst, ependymoma, gangliocytoma, meningioma, neurocytoma, papillary glioneuronal tumour and pleomorphic xanthoastrocytoma. The study was approved by the ethics review board of the University of Erlangen, and all procedures were conducted in accordance with the ethics requirements of the contributing centres. Seizure outcome data of some patients who were included in singlecentre cohort studies have been reported before (Cloppenborg et al. 2019; Bien et al. 2013; Vermeulen et al. 2016; Lamberink et al. 2015b; Baud et al. 2018).

\section{Outcomes}

Individual surgery outcomes were "seizure-freedom" and "both seizure- and drug-freedom". From submitted data, seizure-freedom was defined as Engel class 1 (including subtypes 1a1d) (Engel et al. 1993) or ILAE class 1 and 2 (Wieser et al. 2001), for at least one year at time of outcome determination, and thus allowing for the presence of only auras. Seizure- and drugfreedom was defined as both having an Engel 1/ILAE 1-2 score and complete freedom from AED.

\section{Statistical analysis}

The difference in seizure-freedom between one and five years after surgery was computed for all diagnoses in the subgroup of patients with complete information at these two data points. The difference in seizure-freedom decline between categories was assessed with a chi- 
square test. The association between histopathology diagnosis and seizure-freedom at two years was determined with random-effects logistic regression models. We characterised the association between seizure- and drug-freedom and histopathological diagnosis only at five years postoperatively, because the proportion of drug-free patients increases with the number of years after surgery. Models were corrected for age at surgery, surgery year, location of surgery, duration of epilepsy and heterogeneity between centres. Interaction effects between histopathological diagnosis and duration of epilepsy further improved model fits.

Missing data were imputed by means of multiple imputations using chained equations with predictive mean matching (Van Buuren and Groothuis-Oudshoorn 2011), with twenty imputation sets (Sterne et al. 2009). Imputed dataset regression results were pooled according to the Rubin rule. The age of onset of hippocampal sclerosis patients below the age of 6 was set to missing, as was their duration of epilepsy, which were subsequently imputed. This was done because descriptive analyses revealed that in 35\% (1048/3038) of hippocampal sclerosis cases, collaborators had indicated an age of seizure onset below 6 years, therefore hampering a certain differentiation between a noted age of precipitating febrile seizures or status epilepticus (typically occurring before 6 years of age) and the age of onset of the subsequent temporal lobe seizures. Statistical modelling was done with 'glmmTMB', version 0.2.2.0 and $R$, version 3.4.1. A two-tailed $p$ value of 0.05 or less was considered significant.

Role of the funding source

No funding source had any role in study design, collection, analysis and interpretation of data, writing the report, or the decision to submit the paper for publication.

\section{Results}

Information regarding histopathological diagnosis was available for 9601 epilepsy surgeries between 2000 and 2012, from 18 countries and 37 centres. Re-operations (77 subjects) and patients without clinical follow-up (377 subjects) were excluded, leaving a total cohort of 9147 patients of whom 2952 (33\%) were below the age of 18 years at time of surgery. Age at surgery ranged between $<1$ and 75 years, $48 \%$ were female, and $66 \%$ of surgeries were in the temporal lobe (Table 1). The largest categories of histopathological diagnosis were hippocampal sclerosis (36\%), MCD (25\%), and tumour (20\%) (Table 1).

Of all sixteen variables relevant to the regression analyses, $15 \%$ of data points were missing $(21,380 / 146,352)$, mainly in the antiepileptic drug status at five (59\%), two (40\%) and one year $(36 \%)$, the seizure outcome at five (39\%), two (10\%), and one year (10\%), and the age at onset of epilepsy (18\%) and duration of epilepsy (18\%) (Supplementary Table 1). 
Table 1 | Demographics

\begin{tabular}{|c|c|c|c|}
\hline Variable & $\begin{array}{l}\text { Total cohort } \\
(\mathrm{n}=9147)\end{array}$ & Children $(\mathrm{n}=2952)$ & Adults $(n=5994)$ \\
\hline Female/male & $\begin{array}{l}4429(48 \cdot 4 \%) / 4714 \\
(51 \cdot 6 \%)\end{array}$ & $\begin{array}{l}1382(46 \cdot 8 \%) / 1570 \\
(53 \cdot 2 \%)\end{array}$ & $\begin{array}{l}2960(49 \cdot 4 \%) / 3030 \\
(50 \cdot 6 \%)\end{array}$ \\
\hline Median age at onset (range) & $10(0-74)$ & $2(0-17)$ & $15(0-74)$ \\
\hline Median duration in years (range) & $11(0-64)$ & $4(0-17)$ & $17(0-64)$ \\
\hline Median age at surgery (range) & $27(0-75)$ & $9 \cdot 2(0-17 \cdot 9)$ & $35(18-75)$ \\
\hline \multicolumn{4}{|l|}{ Location of surgery } \\
\hline Temporal & $6018(66 \cdot 4 \%)$ & $1176(40 \cdot 7 \%)$ & $4730(79 \cdot 1 \%)$ \\
\hline Frontal & $1376(15 \cdot 2 \%)$ & $660(22 \cdot 9 \%)$ & $675(11 \cdot 3 \%)$ \\
\hline Occipital & $218(2 \cdot 4 \%)$ & $105(3 \cdot 6 \%)$ & $103(1.7 \%)$ \\
\hline Parietal & $285(3 \cdot 1 \%)$ & $128(4 \cdot 4 \%)$ & $150(2 \cdot 5 \%)$ \\
\hline Multilobar & $1040(11 \cdot 5 \%)$ & $727(25 \cdot 2 \%)$ & $285(4 \cdot 8 \%)$ \\
\hline Hypothalamus & $128(1.4 \%)$ & $90(3 \cdot 1 \%)$ & $36(0 \cdot 6 \%)$ \\
\hline \multicolumn{4}{|l|}{ Side of surgery } \\
\hline Left & $4577(50 \cdot 3 \%)$ & $1463(49.9 \%)$ & $3020(50 \cdot 6 \%)$ \\
\hline Hypothalamus & $128(1.4 \%)$ & $90(3 \cdot 1 \%)$ & $36(0 \cdot 6 \%)$ \\
\hline Right & $4396(48 \cdot 3 \%)$ & $1380(47 \cdot 1 \%)$ & $2913(48 \cdot 8 \%)$ \\
\hline \multicolumn{4}{|l|}{ Histopathological diagnosis } \\
\hline Hippocampal sclerosis & 3260 (35.6\%) & $384(13 \%)$ & 2816 (47\%) \\
\hline $\begin{array}{l}\text { Malformation of cortical } \\
\text { development }\end{array}$ & 2264 (24.8\%) & 1300 (44\%) & 893 (14.9\%) \\
\hline Tumour & 1849 (20.2\%) & 707 (23.9\%) & 1101 (18.4\%) \\
\hline No lesion & 836 (9.1\%) & $223(7.6 \%)$ & $602(10 \%)$ \\
\hline Vascular & 494 (5.4\%) & 109 (3.7\%) & 379 (6.3\%) \\
\hline Scar & 298 (3.3\%) & 143 (4.8\%) & 148 (2.5\%) \\
\hline Encephalitis & $146(1.6 \%)$ & $86(2.9 \%)$ & 55 (0.9\%) \\
\hline
\end{tabular}

\section{Seizure-freedom and AED-freedom over time}

The histopathological diagnosis with most favourable seizure outcome at two years was 'tumour' with 76\% (1239/1635) seizure-free patients, followed by $74 \%$ (328/443) in vascular malformations, $72 \%(2108 / 2948)$ in hippocampal sclerosis, 60\% (1229/2040) in MCD, 60\% $(74 / 124)$ in encephalitis, and 59\% (155/261) in glial scar. Fifty-four percent (396/740) of patients with no histopathological lesion were seizure-free at two years. Outcome varied considerably between diagnostic subcategories (Table 2). 
Table 2 | Seizure-freedom (Engel1/ILAE 1-2) at 1, 2 and 5 years after surgery by histopathological diagnosis.

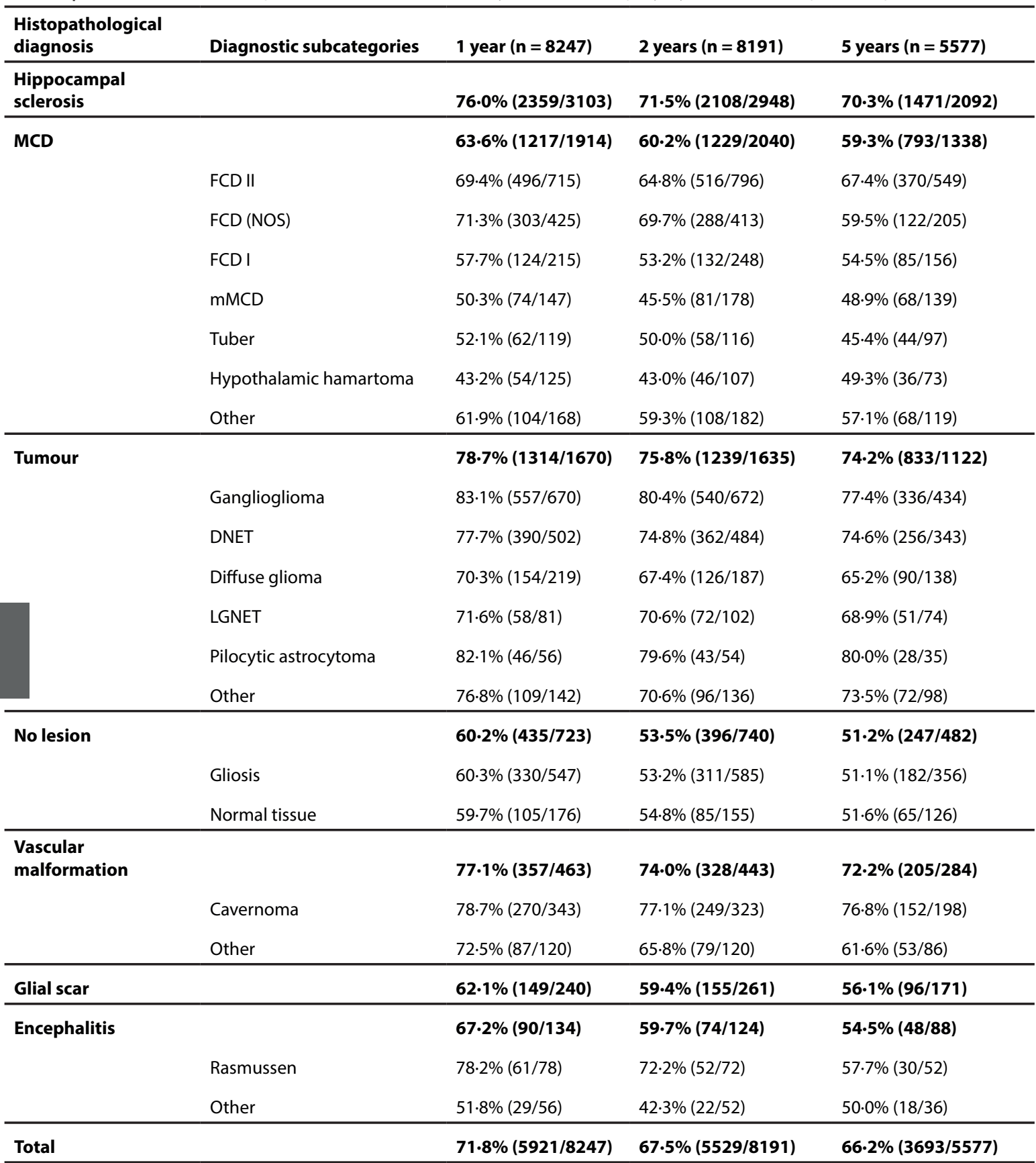

The proportions of the main categories of histopathological diagnosis correspond to Figure 1. DNET = Dysembryoplastic neuroepithelial tumour. FCD I = Focal cortical dysplasia type I. FCD II = Focal cortical dysplasia type II. FCD (NOS) = Focal cortical dysplasia (not otherwise specified). MCD = Malformation of cortical development. mMCD = mild MCD. LGNET = Low-grade neuroepithelial tumour. 
Table 3 | Change in seizure-freedom over the years per histopathological diagnosis in 5077 patients with data available at both 1 year and 5 years postoperatively.

\begin{tabular}{lrrrr}
\hline Histopathological diagnosis & Total & $\begin{array}{r}\text { Number seizure-free } \\
\text { patients 1y }\end{array}$ & $\begin{array}{r}\text { Number seizure- } \\
\text { free patients 5y }\end{array}$ & $\begin{array}{r}\text { Delta seizure- } \\
\text { freedom }\end{array}$ \\
\hline HS & 1969 & 1472 & 1383 & $4 \cdot 5 \%$ \\
MCD & 1169 & 738 & 691 & $4 \cdot 0 \%$ \\
Tumour & 1022 & 791 & 761 & $2 \cdot 9 \%$ \\
No lesion & 398 & 229 & 201 & $7 \cdot 0 \%$ \\
Vascular & 274 & 208 & 200 & $2 \cdot 9 \%$ \\
Scar & 163 & 103 & 91 & $7 \cdot 4 \%$ \\
Encephalitis & 82 & 52 & 45 & $8 \cdot 5 \%$ \\
\hline TOTAL & 5077 & 3593 & 3372 & $4 \cdot 4 \%$ \\
\hline Chisquar
\end{tabular}

Chi-squared test for the columns'total' and 'delta': X-squared $=18.59, \mathrm{df}=6, \mathrm{p}=0.005$. HS $=$ hippocampal sclerosis. $M C D=$ malformation of cortical development.

In patients with the histopathological diagnosis of $M C D$, those with a hypothalamic hamartoma had a two-year seizure-freedom of $43 \%(46 / 107)$ compared to $65 \%(516 / 796)$ in those with FCD II. Figure 1 plots the proportion of seizure-free patients per diagnostic category, from one to five years postoperatively. As a sensitivity analysis, this figure was recreated using the subset of patients for whom seizure outcome was known at all three time points, yielding similar seizure-freedom rates and their change over time (Supplementary Figure 1). The decline in seizure-freedom from one to five postoperative years differed between the categories, with a decrease of $9 \%(7 / 82)$ for those with encephalitis, and $3 \%$ for vascular malformations (8/274) and tumours (30/1022). This difference was statistically significant (Table 3 , chi-squared $=$ $18 \cdot 59$, degrees of freedom $(d f)=6, p=0.005)$.

Multivariable regression analysis (Table 4) revealed that, compared to the histopathological group of tumours, the chance of reaching seizure-freedom at two years was significantly lower for the diagnostic categories no lesion (odds ratio (OR) $0.37,95 \%$ confidence interval (CI) 0.30-0.44) , scar (0.53, 0.36-0.77), encephalitis (0.56, 0.43-0.75), MCD (0.57, 0.49-0.66), and hippocampal sclerosis $(0.85,0.73-0.98)$ (Table 4). Seizure-freedom in tumour patients was not statistically different from that in patients with vascular malformation (0.93, 0.73-1.18) (Table 4). Apart from histopathology, independent positive predictors of seizure-freedom at two years were: surgery in childhood, temporal lobe surgery, and shorter duration of epilepsy.

The proportion of both seizure- and AED-free patients is displayed in Figure 2 and Supplementary Table 2. Seizure- and drug-freedom rates increased over time, varying between $3 \%$ to $9 \%$ at one year postoperatively and $17 \%$ to $43 \%$ at five years (Supplementary Figure 2). After adjusting for potential confounders, the relation between histopathology and 
seizure- and drug-freedom at five years was similar to the relation between histopathology and seizure-freedom (Supplementary Table 3). People with tumours were significantly more often seizure- and drug-free at five years after surgery, compared with patients with no lesion (OR 0.32, 95\% Cl 0.23-0.44), scar (0.33, 0.21-0.53), encephalitis (0.39, 0.24-0.64), MCD (0.54, 0.43-0.69), and hippocampal sclerosis $(0.68,0.56-0.83)$ (Supplementary Table 3). There was no significant difference in seizure- and AED-freedom between patients with tumours and vascular malformations (OR 0.86, 95\% Cl 0.58-1.27) (Supplementary Table 3). Independent positive predictors of seizure- and drug-freedom at five years were - apart from histopathology - younger age, temporal surgery, and shorter epilepsy duration.

The proportion of seizure-freedom varied between centres, with an interquartile range of 6274\% seizure-free patients at five years (Supplementary Figure 3). For seizure- and drug-freedom at five years the variation between centres was much larger, with an interquartile range of 18$37 \%$ (Supplementary Figure 3). This natural variation between centres was effectively modelled with random intercepts.

\section{Differential effect of duration of epilepsy}

Overall, epilepsy duration before surgery was negatively correlated with seizure-freedom outcome (OR 0.99, 95\% Cl 0.98-0.99, p < 0.0001, Table 4), shorter duration correlating with better outcome. The effect of duration on seizure-freedom varied across the different diagnoses. An interaction effect between diagnosis and duration (Supplementary Table 4) significantly improved the model fit (Likelihood ratio test between model with and model without interaction effect between duration of epilepsy and diagnosis, Chi-squared $=39.7$, $d f=6, p<0.0001$ ). Figure 3 visualises the difference in the effect of duration on outcome between the diagnoses. This figure illustrates that, as an example, an adult with a tumour in the temporal lobe has an $83 \%$ chance of seizure-freedom at two years when surgery is performed within a year after epilepsy onset; when surgery is delayed until 20 years after epilepsy onset, this chance is reduced to $74 \%$. A similar pattern is seen for the other diagnoses, except for MCD and hippocampal sclerosis. In the latter two categories, the odds ratio for duration was 1.00 (0.99-1.01) and 1.00 (0.98-1.01), respectively. 
Seizure-freedom after epilepsy surgery

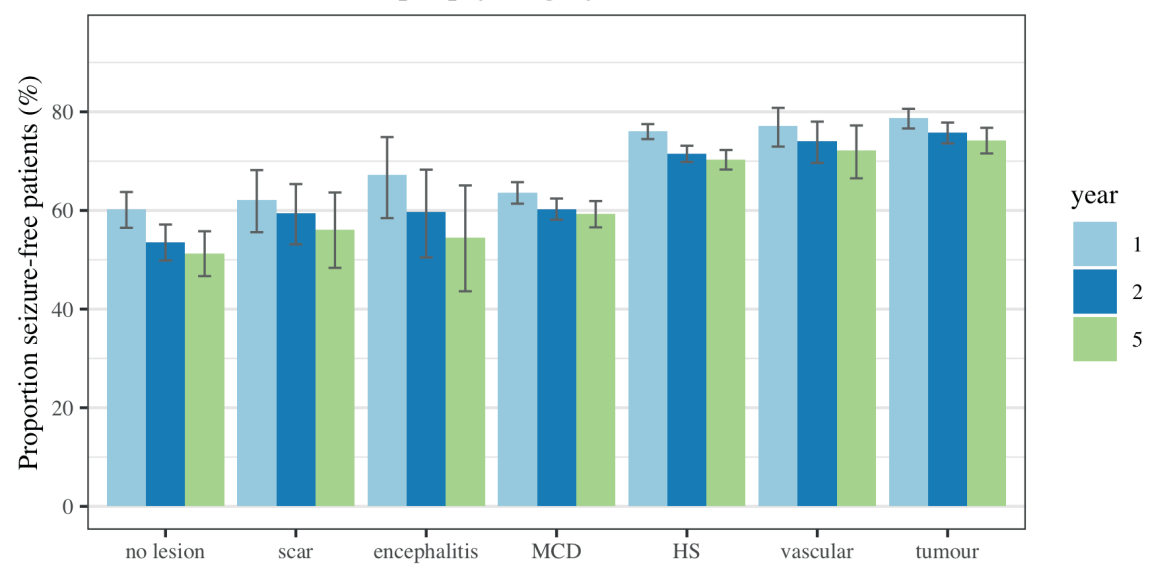

Figure 1 | Proportion of patients who were seizure free (Engel 1/ILAE 1-2) at $1(n=5921 / 8247)$, $2(n=5529 / 8191)$ and $5(n=3693 / 5577)$ years after surgery.

The exact proportions corresponding to this figure are indicated in Table 2. For the same information on cases with information available on all three follow-up time-points, see the complete case Supplementary Figure 1. HS = hippocampal sclerosis. MCD = malformation of cortical development.

Seizure- and AED-freedom after epilepsy surgery

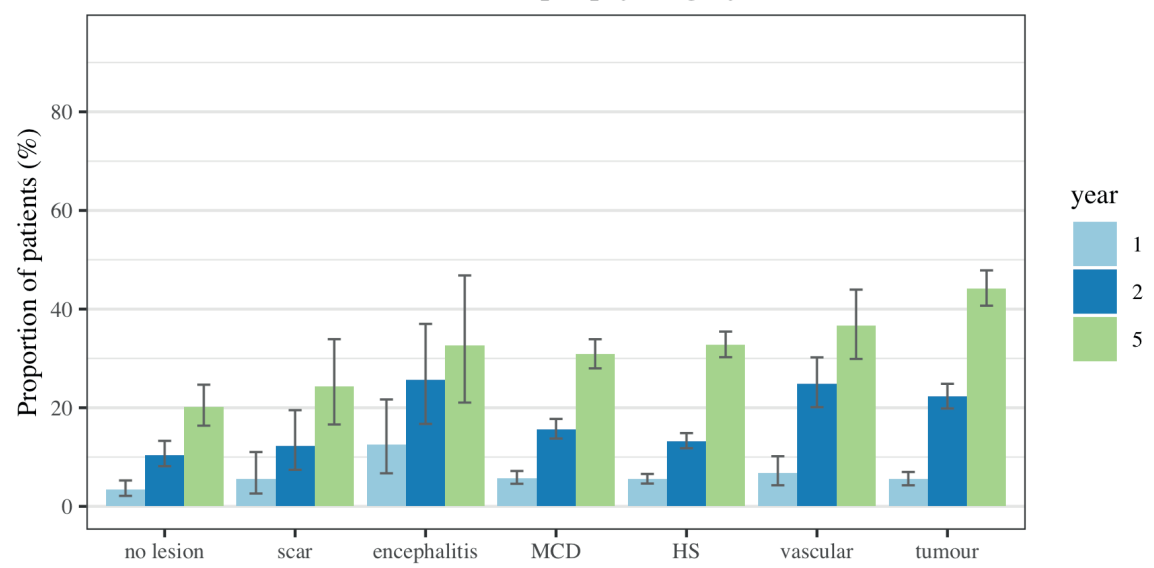

Figure 2 | Proportion of patients who were both seizure free (Engel 1/ILAE 1-2) and had completely discontinued antiepileptic drugs at $1(n=323 / 5861), 2(n=881 / 5461)$ and 5 $(n=1250 / 3753)$ years after surgery.

For the same information on cases with information at three time-points, see the complete case Supplementary Figure 2. HS = hippocampal sclerosis. MCD = malformation of cortical development. 
Table 4 | Multivariable regression model with seizure-freedom (Engel 1/ILAE 1-2) at 2 years after surgery as outcome.

\begin{tabular}{|c|c|c|c|c|}
\hline Variable & Odds ratio & $95 \% \mathrm{Cl}$ & & p value \\
\hline \multicolumn{5}{|c|}{ Histopathological diagnosis } \\
\hline Tumour & 1.00 (ref) & & & \\
\hline Vascular & 0.93 & 0.73 & $1 \cdot 18$ & 0.54 \\
\hline HS & 0.85 & 0.73 & 0.98 & 0.03 \\
\hline MCD & 0.57 & 0.49 & 0.66 & $<0.0001$ \\
\hline Encephalitis & 0.56 & 0.43 & 0.75 & 0.0004 \\
\hline Scar & 0.53 & 0.36 & 0.77 & 0.001 \\
\hline No lesion & 0.37 & 0.30 & 0.44 & $<0.0001$ \\
\hline \multicolumn{5}{|l|}{ Age at surgery } \\
\hline$<18$ years & 1.00 (ref) & & & \\
\hline$\geq 18$ years & 0.80 & 0.69 & 0.92 & 0.002 \\
\hline Surgery year & $1 \cdot 00$ & 0.99 & 1.02 & 0.63 \\
\hline \multicolumn{5}{|l|}{ Location of surgery } \\
\hline Temporal & 1.00 (ref) & & & \\
\hline Frontal & 0.62 & 0.53 & 0.72 & $<0.0001$ \\
\hline Hypothalamus & 0.27 & $0 \cdot 18$ & 0.42 & $<0.0001$ \\
\hline Multilobar & 0.77 & 0.65 & 0.92 & 0.004 \\
\hline Occipital & 0.62 & 0.46 & 0.84 & 0.002 \\
\hline Parietal & 0.70 & 0.53 & 0.92 & 0.01 \\
\hline Duration of epilepsy & 0.99 & 0.98 & 0.99 & $<0.0001$ \\
\hline
\end{tabular}

Duration of epilepsy is the time between the first seizure and surgery in years. Surgery year is the year in which surgery was performed (from 2000 to 2012). HS = hippocampal sclerosis. MCD = malformation of cortical development. 


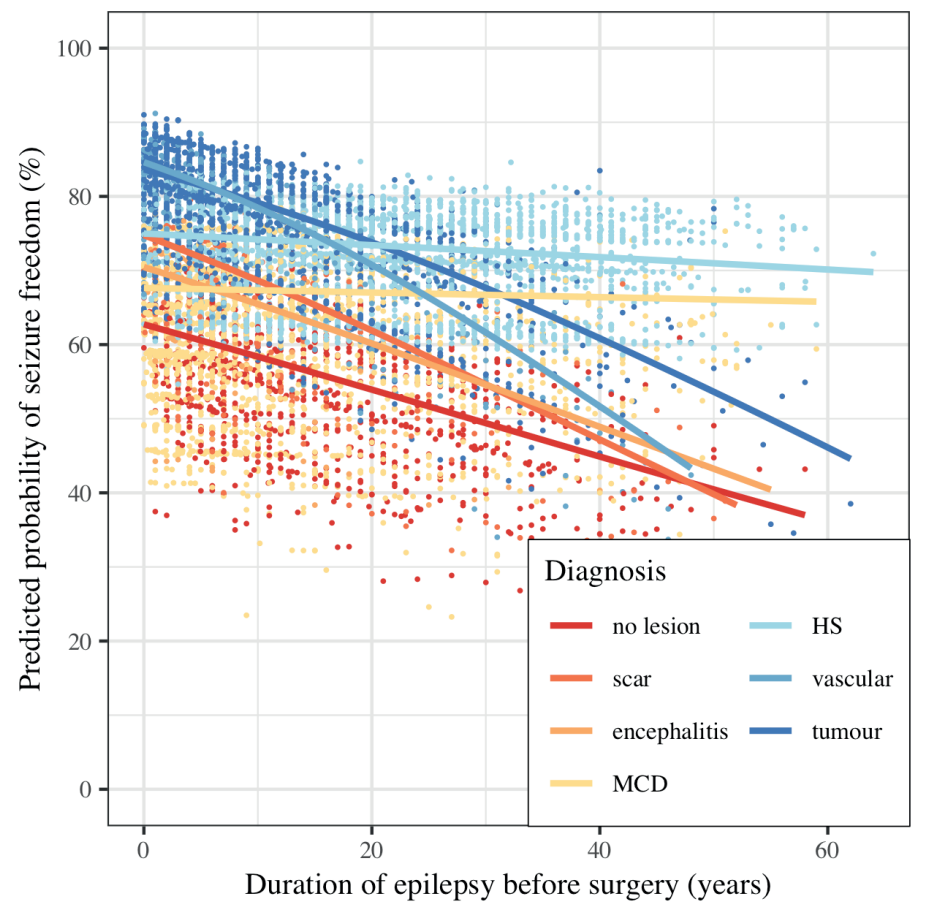

Figure 3 | Predicted probabilities of seizure-freedom (Engel 1/ILAE 1-2) at two years for the different diagnoses, plotted against duration of epilepsy.

Individual dots indicate predicted probability of included patients, based on the model presented in Supplementary Table 4. Lines are the average predicted probability for each histopathological diagnosis (with age at surgery $=$ adult, surgery year $=2007$, and location $=$ temporal). $\mathrm{HS}=$ hippocampal sclerosis. $\mathrm{MCD}=$ malformation of cortical development.

\section{Comparing adults and children}

Overall, children had a better prognosis of seizure-freedom than adults: the odds ratio for seizure-freedom in patients who underwent surgery above the age of 17 years was 0.80 ( $95 \%$ $\mathrm{Cl}$ 0.69-0.92, Table 4). Uncorrected for confounders however, group averages were similar with $67 \%$ seizure-free children (1131/1680) and 66\% seizure-free adults (2475/3728) at five years. In all pathology categories, except MCD and vascular lesions, children had higher proportions of seizure-freedom at five years (Figure 4). To study the effect of age at surgery within the diagnoses, an interaction effect between these variables was added to the regression model (Supplementary Table 5). Only MCD was significantly different from the reference category tumour ( $p$ for interaction 0.02), with no a difference between adults and children regarding seizure-freedom at two years. Figure 5 displays the comparison between children and adults regarding seizure- and drug-freedom, with even larger differences between the two groups. For 
all categories, children were more often seizure- and drug-free, with between 11-32\% (group average 45\%) more seizure and drug-free children as compared to adults (group average 28\%), depending on the diagnosis (Figure 5). The adjusted odds ratio for seizure- and drug-freedom when operated at adult age was 0.56 (95\% Cl 0.45-0.69, Supplementary Table 3).

Seizure-freedom 5 years after epilepsy surgery
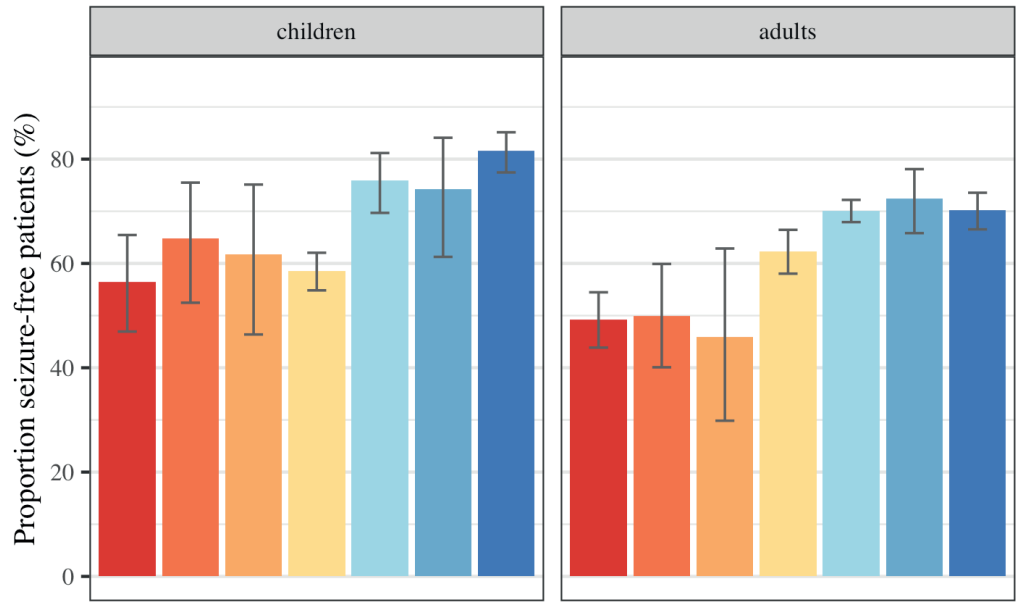

category



Figure 4 | Proportion of patients who were seizure free (Engel 1/ILAE 1-2) at 5 years compared between children $(n=1131 / 1680)$ and adults $(n=2475 / 3728)$

$\mathrm{HS}=$ hippocampal sclerosis. $\mathrm{MCD}=$ malformation of cortical development.

Seizure- and AED-freedom 5 years after epilepsy surgery
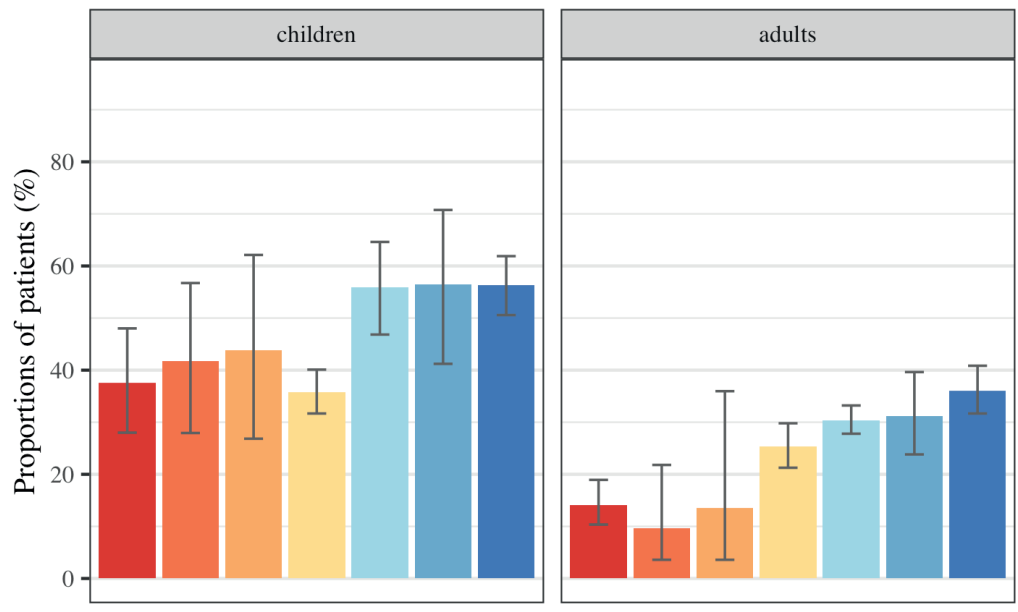

category

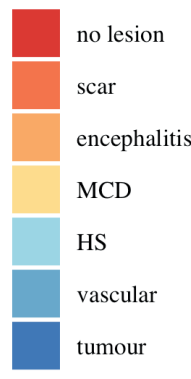

Figure 5 | Proportion of patients who were both seizure free (Engel 1/ILAE 1-2) and completely discontinued antiepileptic drugs at 5 years compared between children $(n=526 / 1175)$ and adults ( $n=703 / 2490)$

$\mathrm{HS}=$ hippocampal sclerosis. $\mathrm{MCD}=$ malformation of cortical development. 


\section{Discussion}

In this large multi-centre cohort study of 9,147 patients who underwent epilepsy surgery across eighteen European countries between 2000 and 2012, histopathological diagnosis was an important and independent determinant of surgery outcome. Average seizure-freedom for all patients was $68 \%$ at two years post-surgery, varying from $76 \%$ in patients with brain tumours to $54 \%$ in those without a microscopically confirmed lesion. From one to five years after surgery, seizure-freedom rates dropped in the range of $3 \%$ to $9 \%$, depending on the underlying brain lesion and least so in patients with vascular lesions and tumours. Independent predictors of seizure-freedom and of seizure- and drug-freedom were histopathology, younger age at surgery, temporal lobe location of surgery, and a shorter disease duration before surgery.

In our study, children had a better prognosis regarding seizure-freedom across all major histopathology diagnoses, and more children discontinued medication after surgery. However, not all previously published studies concur with this observation. A multi-centre study comparing 402 children to 896 adults presented a seizure-freedom rate of $70 \%$ for children and 69\% for adults two years after surgery (Baud et al. 2018). The multivariable odds ratio in that study was not significantly different from 1 . Another single-centre study comparing 690 children with 1305 adults described $58 \%$ of children being seizure-free at two years and $48 \%$ of adults (Cloppenborg et al. 2019). Our study revealed that children undergo more often a resection outside the temporal lobe, less often have a diagnosis of hippocampal sclerosis, and more often suffer from pathologies that are known to have worse outcomes, such as encephalomalacia (histopathologically classified as glial scar). Despite this relatively unfavourable prognostic profile, surgery in childhood was associated with equal surgical outcomes, independent of an inherently shorter duration of epilepsy in this group of patients.

Previous large studies (maximum of 1995 subjects) have analysed the relation between histopathology and surgery outcomes (Alsemari et al. 2014; Baud et al. 2018; Bien et al. 2013; Cloppenborg et al. 2019; Radhakrishnan et al. 2018; Jehi et al. 2015). Reported seizure-freedom was between $49 \%$ and $73 \%$ in these cohorts, at various time intervals after surgery, and the only aetiology that was uniformly characterised in all studies was hippocampal sclerosis. The recognition of all other aetiologies varied considerably across these studies rendering a reliable conclusion of surgery outcome for different brain lesions problematic. Our large collection of data bridges this gap and provides a comprehensive overview of histopathology entities and seizure outcome at 1, 2 and 5 years after surgery. Several studies have suggested that shorter disease duration is a predictive measure for better outcome (Wieser et al. 2003; Janszky et al. 2005; Edelvik et al. 2013; Lamberink et al. 2015a; Jehi et al. 2015), substantiated by a recent aggregate meta-analysis (Bjellvi et al. 2019). We obtained identical results (Figure 3) with two observations in need of further clarification. In the MCD category, duration of epilepsy was not correlated with outcome. Heterogeneity of various histopathology subtypes within the 
MCD category may explain this finding. As an example, the group contains a cohort of 364 patients with a poor outcome pathology (tuber and FCD I), who had a shorter duration of epilepsy (mean 4.6 and 7 years respectively), in contrast with the large group of 1209 good outcome patients (FCD II and FCD NOS) who had a longer duration of epilepsy ( 9 and 10 years respectively). The absence of an effect of duration for the hippocampal sclerosis patients is also difficult to explain, although other studies reported similar results where duration had an effect on outcome except in the case of hippocampal sclerosis (Jehi et al., n.d.; Wieser et al. 2003; Chassoux et al. 2017); only one study showed a possible effect for hippocampal sclerosis patients specifically (Janszky et al. 2005).

In contrast to the other known predictors of surgical outcome, duration of epilepsy is the only factor that can be influenced by decisions made after the diagnosis of drug-resistant epilepsy. Many studies have shown a delay in referral of patients with focal epilepsy to a specialised epilepsy surgery centre (Erba et al. 2012; Kumlien and Mattsson 2010; De Flon et al. 2010; Uijl et al. 2012), with an average time between onset and epilepsy surgery of twenty years in adults (Blümcke et al. 2017). Another factor might be the patients' perspective on epilepsy surgery. A recent study among German epileptologists and patients (Steinbrenner, Kowski, and Holtkamp 2019) found that only one in three patients followed the epileptologists' recommendation to enter presurgical evaluation. This is often caused by fear, possible postoperative handicaps or cognitive deficits. It is also related to the lack of success of surgery in $>25 \%$ of cases. Counselling on the expected benefits and low risks of surgery to patients, and improving referral practices from secondary to tertiary care may shorten the duration until surgery and improve outcomes.

Although $51-74 \%$ of patients were free from disabling seizures (Engel class I), only $20-44 \%$ of them were both seizure- and drug-free at five years. This contrast was more pronounced in adults, where only $10-36 \%$ were seizure- and drug-free at five years, compared to $36-57 \%$ of children. The fact that children withdraw from medication more often and earlier than adults was also observed in a Swedish national study (Edelvik et al. 2013). A major contributing factor for the difference between adults in children are the consequences of AED withdrawal and the risk of seizure recurrence. For adults these consequences may have a much larger impact, for example by losing a driver licence (Bonnett et al. 2011), but also factors like occupation and stigma could play a role. Hence, these differences may not reflect a difference in seizure control without AEDs, but rather the different attitudes between adults and patients.

\section{Strengths and limitations of this study}

This is the largest study on histology findings in epilepsy surgery with outcome data available at 1, 2 and 5 years after surgery with or without AED withdrawal. Including more than 9,000 patients allowed for precise analyses of the effects on seizure outcome for all aetiologies separately, and of the independent effect of duration of epilepsy within subgroups. Epilepsy 
surgery outcomes are usually presented for cohorts of only several hundred patients, limiting the possibilities of subgroup analysis or correcting for multiple confounding factors. Many studies dichotomise, therefore, between temporal and extratemporal lobe surgeries, or assemble smaller aetiologies together into "others". Another strength of our histopathologybased approach is a reliable phenotyping of patients with low chance of misclassification. The only category where misclassification plays a role is the'no lesion' group. However, the majority of patients in this group had TLE without microscopic evidence of hippocampal sclerosis. In a small proportion of the no-lesion group the primary pathological tissue may not have been sent for review or left in place after being disconnected, as often is the case in hemispheric surgeries (i.e. sampling error).Drawing causal inferences from observational data is an inherent difficulty challenging our conclusions on the effect of duration of epilepsy. Interestingly, the beneficial effect of short disease duration remained after correcting for the confounders age at surgery, histopathology, and surgery lobe. Other probable confounders were not available for analysis, however, such as preoperative MRI data, EEG characteristics, completeness of resection and resection volume, medical and seizure history (Ryvlin, Cross, and Rheims 2014; Gracia et al. 2015; Jobst and Cascino 2019; Bell et al. 2017), which may decrease the true effect. Complexity of the epilepsy syndrome may have confounded the relation between duration of epilepsy and outcome. The presurgical evaluation trajectory - and possibly also the time interval before referral to a surgical centre - may have been longer in patients with less straight-forward focal structural epilepsies, in whom the need for more presurgical diagnostic investigations inherently reflects a lower chance of surgical success (Gracia et al. 2015; Ryvlin, Cross, and Rheims 2014).

Due to the retrospective design of the data collection not all clinical parameters were available and data entries have not been standardised. The outcome data were not complete for all three time-points in all patients. The sensitivity analyses using only those patients with all three outcomes available, however, showed no discrepancies when compared to the main results presented in this study. By imputing missing data for the logistic regression this problem is largely overcome (Sterne et al. 2009).

Finally, we used an imperfect measure of seizure freedom. Engel class 1 contains four subdomains, of which only Engel class $1 \mathrm{~A}$ entails complete seizure freedom ever since surgery (similar to ILAE class 1). Formally, we have therefore only reported on "freedom from disabling seizures", which has two consequences for the interpretation of results: (1) in a small proportion of patients with good outcome, we don't know whether surgery with curative intent had been fully successful, and (2) discontinuation of medication is not possible for everyone with an Engel class 1 score, explaining part of the discrepancy between seizure-freedom and drugfreedom. 
In conclusion, this patient cohort from the European Epilepsy Brain Bank allowed for comparison of surgery outcome across all major disease aetiologies. Seizure-freedom dropped from $72 \%$ at one year postoperatively to $66 \%$ at five years, with important differences between histopathological diagnoses. Apart from histopathology, important factors associated with good outcome after surgery are a short duration of epilepsy, young age at surgery, and when the temporal lobe was targeted for surgery. 


\section{SUPPLEMENTARY FIGURES AND TABLES}

\section{Supplementary Table 1 | Missing data.}

\begin{tabular}{|c|c|c|}
\hline Variable & $\begin{array}{r}\text { Number of } \\
\text { datapoints missing }\end{array}$ & $\begin{array}{r}\text { Percent datapoints } \\
\text { missing }\end{array}$ \\
\hline Center & 0 & $0 \%$ \\
\hline Surgery year & 0 & $0 \%$ \\
\hline Histopathological diagnosis & 0 & $0 \%$ \\
\hline Histopathological subdiagnosis & 0 & $0 \%$ \\
\hline Sex & 4 & $0 \%$ \\
\hline Side & 46 & $1 \%$ \\
\hline Location & 82 & $1 \%$ \\
\hline Age at surgery & 201 & $2 \%$ \\
\hline Seizure outcome at 1 year & 900 & $10 \%$ \\
\hline Seizure outcome at 2 years & 956 & $10 \%$ \\
\hline Age at onset & 1686 & $18 \%$ \\
\hline Duration & 1692 & $18 \%$ \\
\hline AED status at 1 year & 3273 & $36 \%$ \\
\hline Seizure outcome at 5 years & 3570 & $39 \%$ \\
\hline AED status at 2 years & 3618 & $40 \%$ \\
\hline AED status at 5 years & 5352 & $59 \%$ \\
\hline Total $\mathrm{n}$ missing variables & 21380 & $15 \%$ \\
\hline
\end{tabular}

Legend to S.Table: Patterns of missing data. Total data points: $146,352$.

AED: antiepileptic drug 
Seizure-freedom after epilepsy surgery



Supplementary Figure 1 | Seizure freedom at 1, 2 and 5 years after surgery in all patients with information at all three time-points $(n=5011)$.

LHS = hippocampal sclerosis. MCD = malformation of cortical development.

Seizure- and AED-freedom after epilepsy surgery

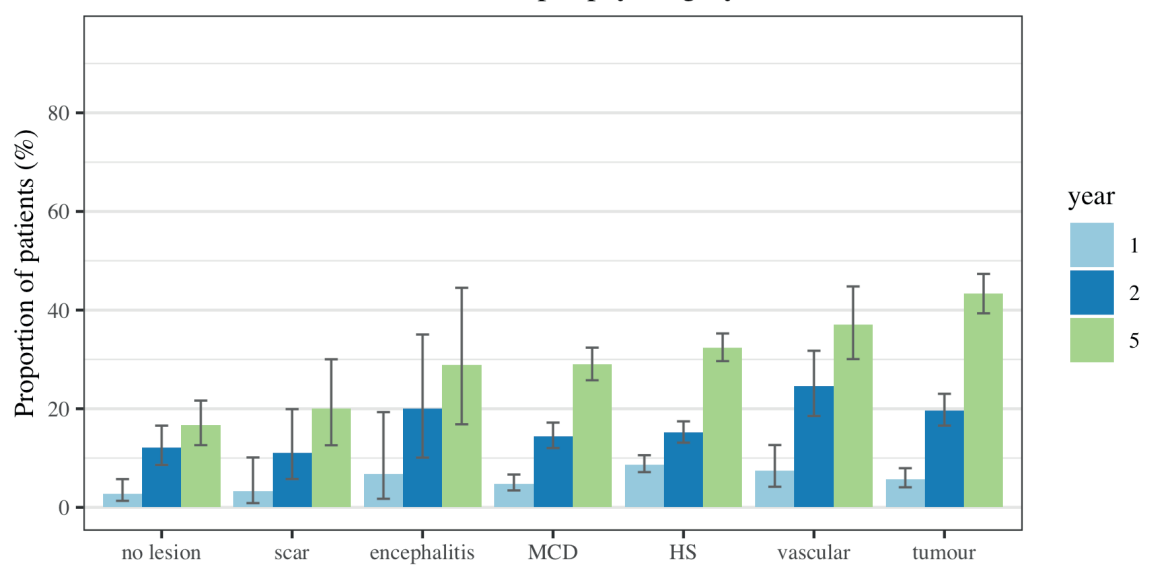

Supplementary Figure 2 | Seizure- and AED freedom at 1, 2 and 5 years after surgery in all patients with information at all three time-points $(n=3669)$.

$\mathrm{HS}=$ hippocampal sclerosis. $\mathrm{MCD}=$ malformation of cortical development. 
Supplementary Table 2 | Proportion of patients who were both seizure free (Engel 1) and had completely discontinued antiepileptic drugs at 1,2 and 5 years after surgery by histopathological diagnosis.

\begin{tabular}{|c|c|c|c|c|}
\hline $\begin{array}{l}\text { Histopathological } \\
\text { diagnosis }\end{array}$ & $\begin{array}{l}\text { Diagnostic } \\
\text { subcategories }\end{array}$ & 1 year $(n=5861)$ & 2 years $(n=5461)$ & 5 years $(n=3753)$ \\
\hline Hippocampal sclerosis & & $5.5 \%(123 / 2228)$ & $13 \cdot 2 \%(257 / 1940)$ & $32 \cdot 8 \%(423 / 1290)$ \\
\hline \multirow[t]{8}{*}{ MCD } & & $5.7 \%(76 / 1323)$ & $15 \cdot 6 \%(208 / 1330)$ & $30.9 \%(299 / 969)$ \\
\hline & Tuber & $3 \cdot 1 \%(2 / 64)$ & $11 \cdot 3 \%(7 / 62)$ & $21 \cdot 6 \%(11 / 51)$ \\
\hline & $\mathrm{mMCD}$ & $0 \%(0 / 102)$ & $9 \cdot 3 \%(11 / 118)$ & $25 \cdot 3 \%(25 / 99)$ \\
\hline & $\begin{array}{l}\text { Hypothalamic } \\
\text { hamartoma }\end{array}$ & $16 \cdot 4 \%(11 / 67)$ & $19 \cdot 6 \%(9 / 46)$ & $21 \cdot 2 \%(7 / 33)$ \\
\hline & FCD I & $2 \cdot 6 \%(4 / 156)$ & $5 \cdot 3 \%(8 / 150)$ & $14 \cdot 8 \%(18 / 122)$ \\
\hline & FCD ॥ & $5 \cdot 8 \%(29 / 504)$ & $15 \cdot 8 \%(86 / 545)$ & $34.9 \%(147 / 421)$ \\
\hline & FCD (NOS) & $6 \cdot 4 \%(20 / 314)$ & $19 \cdot 7 \%(59 / 300)$ & $37 \cdot 0 \%(57 / 154)$ \\
\hline & Other & $8 \cdot 6 \%(10 / 116)$ & $25 \cdot 7 \%(28 / 109)$ & $38 \cdot 2 \%(34 / 89)$ \\
\hline \multirow[t]{7}{*}{ Tumour } & & $5 \cdot 5 \%(64 / 1170)$ & $22 \cdot 3 \%(245 / 1101)$ & $44 \cdot 2 \%(338 / 764)$ \\
\hline & Diffuse glioma & $5 \cdot 8 \%(9 / 155)$ & $148 \cdot 0 \%(17 / 121)$ & $28.4 \%(27 / 95)$ \\
\hline & LGNET & $4.9 \%(2 / 41)$ & $17 \cdot 7 \%(11 / 62)$ & $42 \cdot 3 \%(22 / 52)$ \\
\hline & DNET & $6.9 \%(27 / 392)$ & $21.4 \%(74 / 346)$ & $44 \cdot 7 \%(109 / 244)$ \\
\hline & Ganglioglioma & $3.9 \%(18 / 458)$ & $25 \cdot 2 \%(115 / 457)$ & $51 \cdot 0 \%(149 / 292)$ \\
\hline & Pilocytic astrocytoma & $0 \%(0 / 24)$ & $13 \cdot 6 \%(3 / 22)$ & $37 \cdot 5 \%(6 / 16)$ \\
\hline & Other & $8 \cdot 0 \%(8 / 100)$ & $26 \cdot 9 \%(25 / 93)$ & $38 \cdot 5 \%(25 / 65)$ \\
\hline \multirow[t]{3}{*}{ No lesion } & & $3.4 \%(20 / 593)$ & $10.4 \%(61 / 584)$ & $20 \cdot 2 \%(77 / 381)$ \\
\hline & Gliosis & $3 \cdot 1 \%(14 / 454)$ & $10 \cdot 6 \%(50 / 470)$ & $22 \cdot 1 \%(65 / 294)$ \\
\hline & Normal tissue & $4 \cdot 3 \%(6 / 139)$ & $9 \cdot 6 \%(11 / 114)$ & $13 \cdot 8 \%(12 / 87)$ \\
\hline \multirow[t]{3}{*}{ Vascular malformation } & & $6 \cdot 7 \%(21 / 315)$ & $24 \cdot 8 \%(74 / 298)$ & $36 \cdot 6 \%(70 / 191)$ \\
\hline & Cavernoma & $4 \cdot 8 \%(11 / 228)$ & $23 \cdot 8 \%(51 / 214)$ & $36 \cdot 7 \%(47 / 128)$ \\
\hline & Other & $11 \cdot 5 \%(10 / 87)$ & $27 \cdot 4 \%(23 / 84)$ & $36 \cdot 5 \%(23 / 63)$ \\
\hline Glial scar & & $5.6 \%(8 / 144)$ & $12 \cdot 3 \%(16 / 130)$ & $24 \cdot 3 \%(25 / 103)$ \\
\hline \multirow[t]{3}{*}{ Encephalitis } & & $12 \cdot 5 \%(11 / 88)$ & $25 \cdot 6 \%(20 / 78)$ & $32 \cdot 7 \%(18 / 55)$ \\
\hline & Rasmussen & $13 \cdot 6 \%(8 / 59)$ & $28 \cdot 0 \%(14 / 50)$ & $41 \cdot 7 \%(15 / 36)$ \\
\hline & Other & $10 \cdot 3 \%(3 / 29)$ & $21.4 \%(6 / 28)$ & $15 \cdot 8 \%(3 / 19)$ \\
\hline Total & & $5 \cdot 5 \%(323 / 5861)$ & $16 \cdot 1 \%(881 / 5461)$ & $33 \cdot 3 \%(1250 / 3753)$ \\
\hline
\end{tabular}

The proportions of the main categories correspond to Figure 2.

DNET = Dysembryoplastic neuroepithelial tumour. FCD I = Focal cortical dysplasia type I. FCD II = Focal cortical dysplasia type II. FCD $(\mathrm{NOS})=$ Focal cortical dysplasia (not otherwise specified). $M C D=$ Malformation of cortical development. $\mathrm{mMCD}$ $=$ minimal MCD. LGNET = Low-grade neuroepithelial tumour. 
Supplementary Table 3 | Multivariable logistic regression with seizure- and AED freedom at 5 years after surgery as outcome.

\begin{tabular}{|c|c|c|c|c|}
\hline Variable & Odds ratio & $95 \% \mathrm{Cl}$ & & \\
\hline \multicolumn{5}{|c|}{ Histopathological diagnosis } \\
\hline Tumour & 1.00 (ref) & & & \\
\hline Vascular & 0.86 & $0 \cdot 58$ & $1 \cdot 27$ & 0.45 \\
\hline $\mathrm{HS}$ & 0.68 & $0 \cdot 56$ & 0.83 & 0.0004 \\
\hline MCD & 0.54 & 0.43 & 0.69 & $<0.0001$ \\
\hline Encephalitis & 0.39 & $0 \cdot 24$ & 0.64 & 0.0002 \\
\hline Scar & 0.33 & 0.21 & 0.53 & $<0.0001$ \\
\hline No lesion & 0.32 & $0 \cdot 23$ & 0.44 & $<0.0001$ \\
\hline \multicolumn{5}{|l|}{ Age at surgery } \\
\hline$<18$ years & 1.00 (ref) & & & \\
\hline$\geq 18$ years & 0.56 & 0.45 & 0.69 & $<0.0001$ \\
\hline Surgery year & 1.00 & 0.98 & 1.02 & 0.99 \\
\hline \multicolumn{5}{|l|}{ Location of surgery } \\
\hline Temporal & 1.00 (ref) & & & \\
\hline Frontal & 0.77 & 0.63 & 0.94 & 0.01 \\
\hline Hypothalamus & 0.42 & 0.24 & 0.74 & 0.003 \\
\hline Multilobar & 1.06 & $0 \cdot 84$ & $1 \cdot 34$ & 0.63 \\
\hline Occipital & 0.64 & 0.39 & 1.03 & 0.07 \\
\hline Parietal & 0.91 & 0.65 & $1 \cdot 28$ & 0.59 \\
\hline Duration of epilepsy & 0.97 & 0.97 & 0.98 & $<0.0001$ \\
\hline
\end{tabular}

Duration of epilepsy is the time between the first seizure and surgery in years. Surgery year is the year in which surgery was performed.

$\mathrm{HS}=$ hippocampal sclerosis. $\mathrm{MCD}=$ malformation of cortical development. 


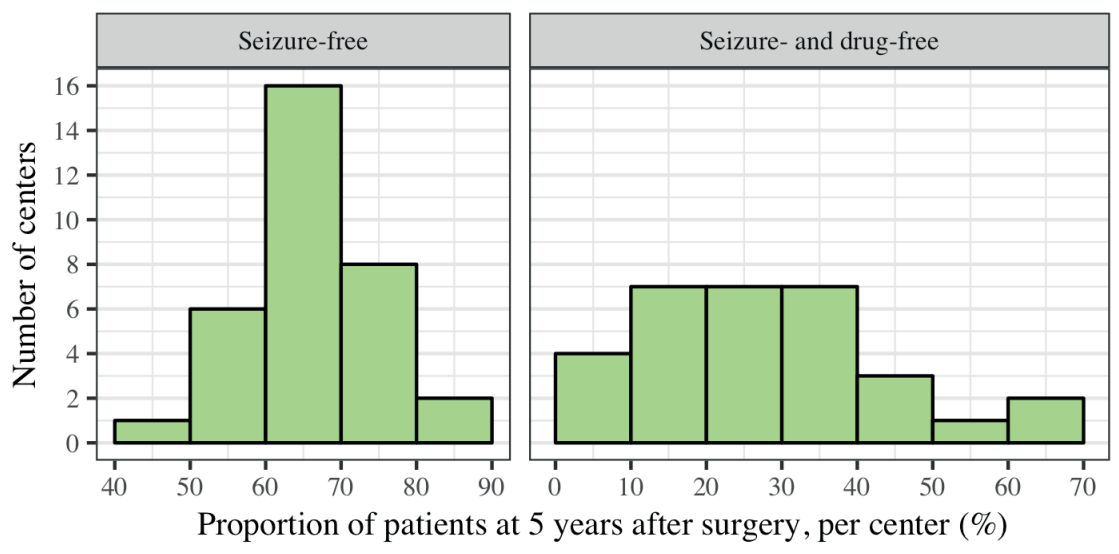

\section{Supplementary Figure 3 | Histogram of surgery outcomes per centre.}

For every centre, the proportion of seizure free and seizure-, and drug free patients was computed. Seizure freedom data at five years was available for 33 centres, and 31 centres had data available regarding seizure-, and drug freedom at five years. 
Supplementary Table 4 | Multivariable logistic regression with seizure-freedom at 2 years after surgery as outcome, including an interaction effect between diagnosis and duration of epilepsy.

\begin{tabular}{|c|c|c|c|c|c|}
\hline Variable & Odds ratio & $95 \% \mathrm{Cl}$ & & $p$ value & p for interaction \\
\hline \multicolumn{6}{|l|}{ Histopathological diagnosis } \\
\hline Tumour & 1.00 (ref) & & & & \\
\hline Vascular & 0.91 & 0.71 & $1 \cdot 17$ & 0.47 & \\
\hline HS & 0.85 & 0.73 & 0.99 & 0.04 & \\
\hline MCD & 0.62 & 0.53 & 0.72 & $<0.0001$ & \\
\hline Encephalitis & 0.58 & 0.43 & 0.77 & 0.0002 & \\
\hline Scar & 0.52 & 0.32 & 0.84 & 0.007 & \\
\hline No lesion & 0.39 & 0.32 & 0.47 & $<0.0001$ & \\
\hline \multicolumn{6}{|l|}{ Age at surgery } \\
\hline$<18$ years & $1.00($ ref $)$ & & & & \\
\hline$\geq 18$ years & 0.80 & 0.69 & 0.93 & 0.003 & \\
\hline Surgery year & 1.00 & 0.99 & 1.02 & 0.74 & \\
\hline \multicolumn{6}{|l|}{ Location of surgery } \\
\hline Temporal & 1.00 (ref) & & & & \\
\hline Frontal & 0.60 & 0.52 & 0.70 & $<0.0001$ & \\
\hline Hypothalamus & 0.27 & 0.18 & 0.41 & $<0.0001$ & \\
\hline Multilobar & 0.77 & 0.64 & 0.91 & 0.003 & \\
\hline Occipital & 0.61 & 0.45 & 0.83 & 0.001 & \\
\hline Parietal & 0.68 & 0.52 & 0.89 & 0.005 & \\
\hline Duration (for tumour) & 0.97 & 0.96 & 0.98 & $<0.0001$ & Reference \\
\hline Duration (for vascular) & 0.96 & 0.94 & 0.98 & 0.0004 & 0.33 \\
\hline Duration (for HS) & 1.00 & 0.98 & 1.01 & 0.53 & 0.0001 \\
\hline Duration (for MCD) & $1 \cdot 00$ & 0.99 & 1.01 & 0.84 & 0.0001 \\
\hline Duration (for encephalitis) & 0.97 & 0.95 & 0.99 & 0.02 & 0.99 \\
\hline Duration (for scar) & 0.98 & 0.94 & 1.02 & 0.31 & 0.76 \\
\hline Duration (for no lesion) & 0.98 & 0.97 & 1.00 & 0.03 & 0.17 \\
\hline
\end{tabular}

Duration of epilepsy is the time between the first seizure and surgery in years. Surgery year is the year in which surgery was performed. $P$ for interaction: when $<0.05$, the conclusion is that the effect of duration is significantly different from the effect in the reference category.

$\mathrm{HS}=$ hippocampal sclerosis. $\mathrm{MCD}=$ malformation of cortical development. 
Supplementary Table 5 | Multivariable regression model with seizure-freedom at 2 years after surgery as outcome, using all potential confounders and an interaction between age and histopathological diagnosis as independent variables.

\begin{tabular}{|c|c|c|c|c|c|}
\hline Variable & Odds ratio & $95 \% \mathrm{Cl}$ & & p value & p for interaction \\
\hline \multicolumn{6}{|l|}{ Histopathological diagnosis } \\
\hline Tumour & 1.00 (ref) & & & & \\
\hline Vascular & 0.67 & 0.38 & $1 \cdot 18$ & 0.17 & \\
\hline HS & 0.84 & 0.60 & $1 \cdot 17$ & 0.30 & \\
\hline MCD & 0.48 & 0.36 & 0.64 & $<0.0001$ & \\
\hline Encephalitis & 0.56 & 0.34 & 0.92 & 0.02 & \\
\hline Scar & 0.72 & 0.34 & 1.48 & 0.37 & \\
\hline No lesion & 0.45 & 0.31 & 0.67 & 0.0001 & \\
\hline Surgery year & 1.00 & 0.99 & 1.02 & 0.82 & \\
\hline \multicolumn{6}{|l|}{ Location of surgery } \\
\hline Temporal & 1.00 (ref) & & & & \\
\hline Frontal & 0.61 & 0.52 & 0.71 & $<0.0001$ & \\
\hline Hypothalamus & $0 \cdot 28$ & 0.19 & 0.43 & $<0.0001$ & \\
\hline Multilobar & 0.76 & 0.64 & 0.91 & 0.003 & \\
\hline Occipital & 0.62 & 0.46 & 0.84 & 0.002 & \\
\hline Parietal & 0.68 & 0.52 & 0.90 & 0.006 & \\
\hline Duration (for tumour) & 0.97 & 0.96 & 0.98 & $<0.0001$ & Reference \\
\hline Duration (for vascular) & 0.96 & 0.93 & 0.98 & 0.0004 & 0.19 \\
\hline Duration (for HS) & 1.00 & 0.98 & 1.01 & 0.60 & 0.0007 \\
\hline Duration (for MCD) & 0.99 & 0.97 & 1.00 & $0 \cdot 16$ & 0.07 \\
\hline Duration (for encephalitis) & 0.97 & 0.94 & 1.00 & 0.07 & 1.00 \\
\hline Duration (for scar) & 0.99 & 0.95 & 1.04 & 0.83 & 0.37 \\
\hline Duration (for no lesion) & 0.99 & 0.97 & 1.01 & 0.20 & $0 \cdot 10$ \\
\hline Adult age at surgery (for tumour) & 0.71 & 0.54 & 0.94 & 0.02 & Reference \\
\hline Adult age at surgery (for vascular) & 1.05 & 0.56 & 1.97 & 0.88 & 0.23 \\
\hline Adult age at surgery (for HS) & 0.74 & 0.51 & 1.08 & $0 \cdot 12$ & 0.83 \\
\hline Adult age at surgery (for MCD) & $1 \cdot 14$ & 0.78 & $1 \cdot 68$ & 0.50 & 0.02 \\
\hline $\begin{array}{l}\text { Adult age at surgery (for } \\
\text { encephalitis) }\end{array}$ & 0.73 & 0.37 & 1.42 & 0.35 & 0.95 \\
\hline Adult age at surgery (for scar) & 0.40 & 0.17 & 0.95 & 0.04 & 0.20 \\
\hline Adult age at surgery (for no lesion) & 0.58 & 0.36 & 0.91 & 0.02 & 0.37 \\
\hline
\end{tabular}

Duration of epilepsy is the time between the first seizure and surgery in years. Surgery year is the year in which surgery was performed. $P$ for interaction: when $<0.05$, the conclusion is that the effect of $x$ (in this case duration, and adult vs. childhood age at surgery) is significantly different from the effect in the reference category.

$\mathrm{HS}=$ hippocampal sclerosis. $\mathrm{MCD}=$ malformation of cortical development. 


\section{APPENDIX}

\section{Collaborators of the EEBB consortium}

Floor Jansen, Frans Leijten, Peter van Rijen, Wim G.M. Spliet, Angelika Mühlebner, Burkhard S Kasper, Susanne Fauser, Tilman Polster, Thilo Kalbhenn, Daniel Delev, Andrew McEvoy, Anna Miserocchi, Elisabeth Landré, Bares Turak, Pascale Varlet, Sarah Ferrand-Sorbets, Martine Fohlen, Christine Bulteau, Anna Edelvik, Mukesch Johannes Shah, Christian Scheiwe, Eva Gutierrez Delicado, Martin Tisdall, Christin Eltze, Serdar Akkol, Kaancan Deniz, Buge Oz, Hans Holthausen, Till Hartlieb, Martin Staudt, Sara Casciato, Pier Paolo Quarato, Felice Giangaspero, Nathalie Streichenberger, Marc Guenot, Jean Isnard, Antonio Valentin, Amanda Chang, Nandini Mullatti, Josef Zamecnik, Jana Zarubova, Martin Tomasek, Arto Immonen, Anni Saarela, Tuomas Rauramaa, Johannes Alexander Lobrinus, Kristof Egervari, Shahan Momjian, Elisabeth Hartl, Hannah Lohr, Judith Kroell, Lynn Vermeulen, Evy Cleeren, Pavel Vlasov, Antonia Kozlova, Alexey Vorobyev, Gudrun Goeppel, Sharon Samueli, Thomas Czech, Johannes Hainfellner, Gertraud Puttinger, Gabriele Schwarz, Harald Stefanits, Serge Weis, Roberto Spreafico, Flavio Villani, Laura Rossini, Anke Hermsen, Susanne Knake, Christopher Nimsky, Barbara Carl, Anezka Belohlavkova, Barbora Benova, Jeroen Bisschop, Albert Colon, Vivianne van KranenMastenbroek, Rob P.W. Rouhl, Govert Hoogland, Jordi Rumiá, Alia Ramírez-Camacho, Santiago Candela-Cantó, Karine Ostrowsky-Coste, Eleni Panagiotakaki, Alexandra Montavont, Pascale Keo Kosal, Zeynep Gokce-Samar, Clara Milleret, Anna Maria Buccoliero, Flavio Giordano, Vlatko Sulentic, Goran Mrak, Andrej Desnica, Giusy Carfi'Pavia, Alessandro De Benedictis, Carlo Efisio Marras, Vladimir Bascarevic, Nikola Vojvodic, Aleksandar Ristic, Olinda Rebelo, Angel AledoSerrano, Irene Garcia-Morales, Carla Anciones. 


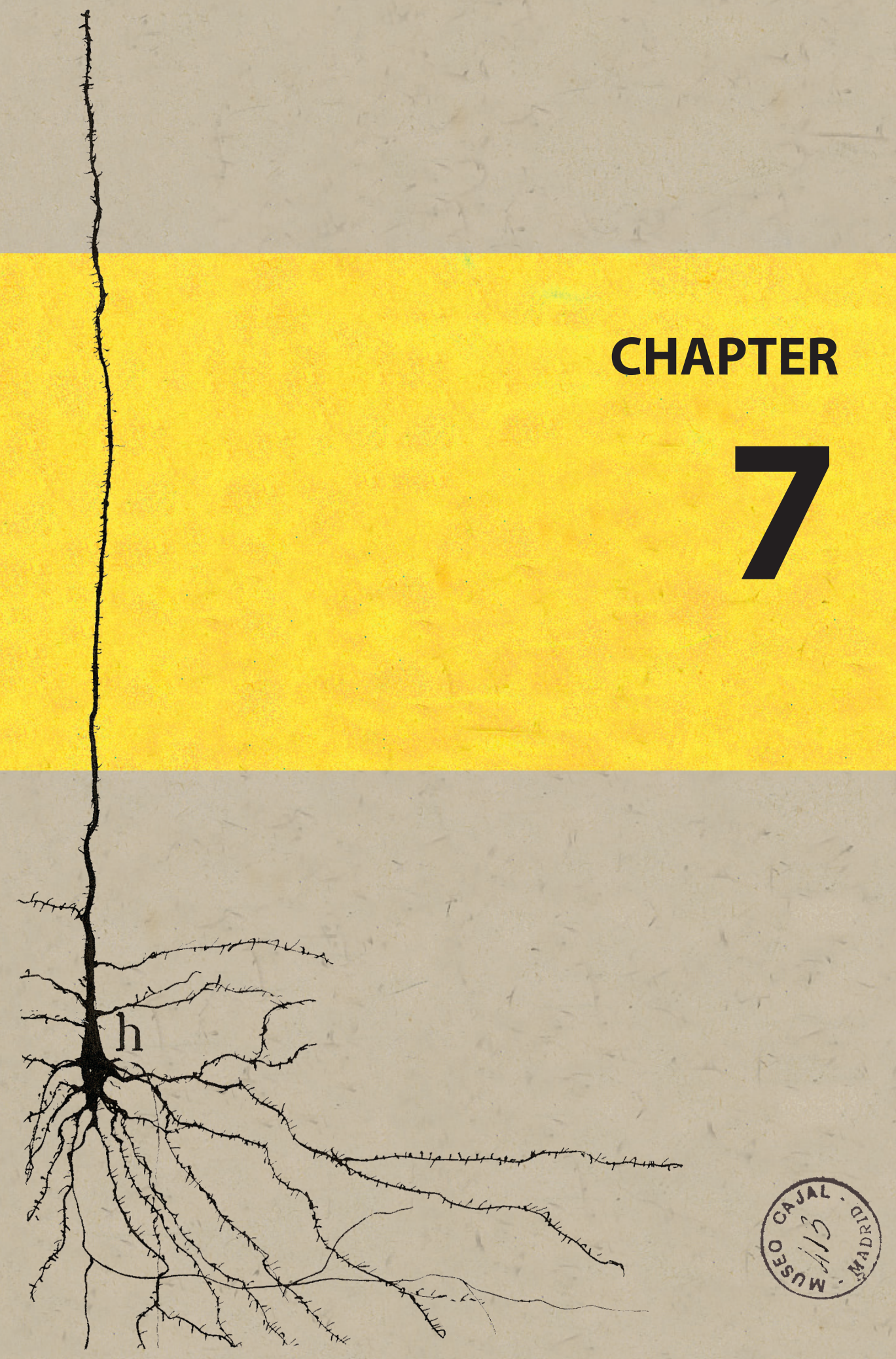




\title{
Cognitive consequences of early versus late antiepileptic drug withdrawal after pediatric epilepsy surgery, the TimeToStop (TTS) trial: study protocol for a randomized controlled trial
}

\author{
Boshuisen $\mathrm{K}^{*}$ \\ Lamberink HJ* \\ van Schooneveld MM \\ Cross JH \\ Arzimanoglou A \\ van der Tweel I \\ Geleijns K \\ Uiterwaal CS \\ Braun KP \\ * Contributed equally
}

Trials. 2015;16:482 


\section{Abstract}

Background: The goals of intentional curative pediatric epilepsy surgery are to achieve seizure-freedom and antiepileptic drug (AED) freedom. Retrospective cohort studies have indicated that early postoperative AED withdrawal unmasks incomplete surgical success and AED dependency sooner, but not at the cost of long-term seizure outcome. Moreover, AED withdrawal seemed to improve cognitive outcome. A randomized trial is needed to confirm these findings. We hypothesized that early AED withdrawal in children is not only safe, but also beneficial with respect to cognitive functioning.

Design: This is a multi-center pragmatic randomized clinical trial to investigate whether early AED withdrawal improves cognitive function, in terms of attention, executive function and intelligence, quality of life and behavior, and to confirm safety in terms of eventual seizure freedom, seizure recurrences and "seizure and AED freedom." Patients will be randomly allocated in parallel groups (1:1) to either early or late AED withdrawal. Randomization will be concealed and stratified for preoperative IQ and medical center. In the early withdrawal arm reduction of AEDs will start 4 months after surgery, while in the late withdrawal arm reduction starts 12 months after surgery, with intended complete cessation of drugs after 12 and 20 months respectively. Cognitive outcome measurements will be performed preoperatively, and at 1 and 2 years following surgery, and consist of assessment of attention and executive functioning using the EpiTrack Junior test and intelligence expressed as IQ (Wechsler Intelligence Scales). Seizure outcomes will be assessed at 24 months after surgery, and at 20 months following start of AED reduction. We aim to randomize 180 patients who underwent anticipated curative epilepsy surgery below 16 years of age, were able to perform the EpiTrack Junior test preoperatively, and have no predictors of poor postoperative seizure prognosis (multifocal magnetic resonance imaging (MRI) abnormalities, incomplete resection of the lesion, epileptic postoperative electroencephalogram (EEG) abnormalities, or more than three AEDs at the time of surgery).

Discussion: Growing experience with epilepsy surgery has changed the view towards postoperative medication policy. In a European collaboration, we designed a multi-center pragmatic randomized clinical trial comparing early with late AED withdrawal to investigate benefits and safety of early AED withdrawal. The TTS trial is supported by the Dutch Epilepsy Fund (NL 08-10) ISRCTN 88423240, 08/05/2013. 


\section{Background}

For children with refractory epilepsy, epilepsy surgery is a successful and widely accepted therapeutic option with postoperative seizure freedom rates ranging from 41 to $93 \%$ (Spencer and Huh 2008; Ryvlin, Cross, and Rheims 2014). In patients who have been operated on and who have reached seizure freedom the ultimate goal is to discontinue antiepileptic drug (AED) use and to improve developmental capacities (Shields 2000; Cross 2002; Van Schooneveld and Braun 2013). AEDs are known to have cognitive side-effects, particularly in children. Most affected cognitive functions are attention, vigilance and psychomotor speed (Loring and Meador 2004; Meador 2002; Lossius et al. 2008; Skirrow et al. 2011). AED reduction has been reported to improve cognitive processing under time pressure (Lossius et al. 2008; Hessen et al. 2006), psychomotor speed and alertness (AP Aldenkamp et al. 1993, 1998; Van Schooneveld et al. 2013), processing speed (Hallböök et al. 2013), verbal memory (Meekes et al. 2013) and Intelligence Quotient (IQ) (Skirrow et al. 2011). The growing body of evidence that cognitive functioning improves after AED withdrawal has increased awareness of the possible benefits of the earliest possible withdrawal of AEDs after epilepsy surgery. Two recently published studies (Boshuisen et al. 2012; Yardi et al. 2014) showed that early withdrawal of drug treatment unmasks incomplete surgical success and AED dependency sooner, but not at the cost of worse long-term seizure outcome. Thus, early AED withdrawal would discern the few that require further AEDs from the many that can safely stop, without changing the chance of regaining seizure freedom. A pressing question is whether early AED withdrawal improves cognitive outcome measures, as compared to current care with usually later AED withdrawal. To answer that question we designed a pragmatic randomized clinical trial to investigate the benefits and safety of early AED withdrawal after epilepsy surgery in children. We hypothesize that 1) children who discontinue AED's early have better cognitive scores, behavior and quality of life than those who discontinue late, particularly at 1 year after surgery (when 1 group is without AEDs and the other is still on AEDs), and maybe persisting from then on, and 2) early AED withdrawal is safe and does not cause more recurrences that are unresponsive to restart of medication, than late withdrawal.

\section{Study objectives}

The primary objective is to investigate whether early AED withdrawal improves cognitive function, in terms of attention and executive functions, compared to late AED reduction. The secondary objectives are to assess (improvement in) IQ, and safety, in terms of seizure recurrences, long-term seizure freedom and "seizure and AED freedom" of early versus late AED withdrawal. Seizure freedom will be defined as complete seizure freedom (including auras), expressed as Engel 1A or ILAE class 1 for at least 1 year (Engel, Van Ness, et al. 1993; Engel and International League Against Epilepsy 2001), and "seizure and AED freedom" as being seizure free without medication for at least 1 year. In addition, we will compare behavior, quality of life and other neuropsychological outcomes between the two AED withdrawal strategies. 


\section{Methods/Design}

\section{Study design}

The TimeToStop trial will be a European multicenter pragmatic randomized clinical trial. In the Netherlands the trial has been approved by the Medical Ethical Committee of the University Medical Center Utrecht, which is the leading ethical committee. Other participating centers are in the process of protocol submission and have not yet obtained definite approval. Only then, local patient recruitment will start. For every patient who will be included in the trial we will obtain informed consent. In the TimeToStop trial, index intervention is early withdrawal: AED withdrawal is started 4 months after surgery and completed within 8 months after start of withdrawal (thus, at latest 12 months postoperatively). Reference intervention is late AED withdrawal: tapering off medication starts at 12 months after surgery and completion should be within 20 months after surgery. For both treatment arms, the total tapering period is set at 8 months maximum and clinicians may decide which AED they want to taper first and at what speed they will reduce each individual drug. A tapering period of 8 months is considered reasonable, as from the retrospective TimeToStop (TTS) study (Boshuisen et al. 2012) it could be calculated that patients who fulfill the inclusion criteria used on average 1.7 AEDs prior to surgery (range $0-3$ ). The late (reference) starting point of AED tapering - i.e. 12 months - is based on current practice; the median interval between surgery and drug reduction in our retrospective European study was 12.5 months (Boshuisen et al. 2012). The 4 months'time-point in the early withdrawal group is selected mainly because of logistical reasons. First, informing parents and recruiting patients needs time. Second, inclusion requires proof of early surgical success, which needs a certain time window to discriminate between acute postoperative (running-down) seizures and true seizure recurrence. Third, in some children clinicians may want to prove completeness of resection by performing a postoperative magnetic resonance imaging (MRI), for which a minimum follow-up time of a few months is required. For both intervention strategies, the treating physician is allowed to start tapering earlier if patients experience unacceptable side effects of prescribed AEDs. Rescue medication that is started preoperatively and withdrawn shortly after surgery, can be discontinued according to existing local routine practice, and will not be investigated in this study.

The trial's primary outcome measure at 1 year after surgery is cognitive functioning, in terms of attention and executive functioning, which will be assessed using the EpiTrack Junior (C Helmstaedter et al. 2010). As secondary cognitive outcome measure, intelligence will be tested with the Wechsler Preschool and Primary Scales, the Wechsler Intelligence Scale for children, or the Wechsler Adult Intelligence scale, depending on the children's age and capabilities; scores derived are Verbal (VIQ), Performal (PIQ) and Full-scale IQ, perceptual organization or reasoning and information processing speed index scores. We will measure baseline neuropsychological status maximum 3 months before surgery ( $\mathrm{t} 1$ ), and thereafter at 12 months ( \pm 2 months) (t2) and 24 months ( \pm 2 months) ( $\mathrm{t} 3$ ) postoperatively. Patients and/or parents will at all 
neuropsychological test points complete three types of questionnaires to assess the secondary outcome measures: the Child Behavior Checklist (CBCL) and the the Hague Side Effects Scale (HASES) (Carpay et al. 2002) will be completed by the parents, and the Pediatric Quality of Life InventoryTM (PedsQL ${ }^{\mathrm{TM}}$ ) will be completed by both parents and patients. This design will allow us to assess whether cognitive performance is better in the early AED-withdrawal group, with patients being AED-free at 1 year following surgery, compared to the group that is still on full medication at the first postoperative time point. It will also enable the study of differences at a later follow-up time point, when both groups have completely discontinued medication ( $\mathrm{t} 3$ ) (Fig. 1). Seizure outcome will be assessed at the moment of randomization, as seizure freedom is an inclusion criterion, at 24 months after surgery, and at 20 months following start of AED withdrawal. This design allows us to assess differences in seizure outcome at equal AED-free intervals for both groups, and at 2 years after surgery irrespective of length of AED freedom.

Patients will be allocated to treatment strategy by concealed blocked randomization and randomization will be stratified for preoperative IQ score and medical center. The primary goal of randomization is to achieve an AED treatment duration contrast. Therefore, physicians and patients allocated to an arm will be instructed to comply with that strategy. However, these intervention strategies will to a certain extent be in compliance with clinical practice, and allow "protocol violations" for the following instances: if patients have seizure recurrences in the period before scheduled AED withdrawal, they do not have to start AED withdrawal. The same applies to seizure recurrences that occur after start of tapering medication. Patients can restart medication and do not have to taper medication again, regardless of planned medication status. Notably, whatever "protocol deviations" will occur, patients will be analyzed as randomized (intention-to-treat principle).

\section{Inclusion criteria}

In order to be eligible a subject must meet all of the following criteria:

- Younger than 16 years at surgery, with focal non-idiopathic epilepsy

- Native speaker in the language in which the neuropsychological tests have to be taken

- Able to perform an EpiTrack Junior (C Helmstaedter et al. 2010) test preoperatively

- Underwent intentionally curative epilepsy surgery

- After surgery, the treating physician considers withdrawal of AEDs, with the intention to completely discontinue medication

- The treating physician, the patient (if capable), and the parents agree with randomization in either arm of the study

- Postoperative seizure freedom was achieved (with the exception of so-called running down seizures not outlasting 2 weeks)

- Written informed consent of children and both parents or caregivers of children older than 12 years, and of both parents or caregivers of children below that age 


\section{Exclusion criteria}

A subject who meets any of the following criteria will be excluded from participation:

- A contraindication to be randomized to either of the two withdrawal strategies

- The treating physician does not want to discontinue all AEDs within a maximum time frame of 8 months as prescribed in the study protocol

- Multifocal MRI abnormalities, known incomplete resection of the anatomical or epileptogenic lesion certified before randomization (if considered necessary by the treating physician a postoperative MRI may be performed) and, if a postoperative electroencephalogram (EEG) is performed before randomization (at the discretion of the treating physician), epileptic EEG abnormalities. These are the most important risk factors of seizure recurrence or unfavorable long-term seizure outcome (Boshuisen et al. 2012)

- Use of more than three AEDs at the time of surgery. The reason is that clinicians can then not be expected to want to wait for 12 months in the late withdrawal arm to reduce the first AED in these patients. Furthermore, withdrawing 4 or more AEDs within 8 months may be difficult to achieve

- Patients who are on a ketogenic diet or have a vagal nerve stimulator implanted

- If surgery is primarily intended as "tumor surgery" (a growing epileptogenic lesion was the indication for surgery) and not as epilepsy surgery

\section{Excluded patients}

Participating centers will provide brief anonymized information about all children who are operated on, including those who were not in the trial, to document reasons for exclusion and the overall proportion of eligible patients, allowing the assessment of generalizability of the trial results. We will only report aggregated data of excluded patients.

\section{Statistical analysis}

The general approach towards analysis will be on an intention-to-treat basis, so patients randomized will be analyzed and accounted for as allocated, independent of later compliance. For all analyses described below, we will add baseline adjusted analyses using various regression techniques (linear regression for continuous and logistic regression for binary outcomes). For these adjustments to increase statistical precision, we will use propensity score methods or inverse variance weighting methods.

Prior to analyses described below, we will deal with missing values by formal accepted multiple imputation methods, for reasons of optimizing statistical precision but more importantly because particularly loss to follow-up may be expected not to occur randomly in this trial. 




Figure 1 | Trial design.

Flowchart of trial design; NPA, neuropsychological assessment 


\section{Outcome parameters}

Cognitive outcome: differences in EpiTrack scores, IQ scores and other continuous outcome measures of neuropsychological assessment will be analyzed using independent samples t-tests or other tests depending on distributions. EpiTrack and IQ differences will be assessed cross-sectionally to answer the primary research question. Additionally, longitudinal changes in EpiTrack measures and IQ compared to preoperatively will be assessed between both treatment arms using linear mixed models. We will calculate a number needed to treat, expressed as the number of patients required to taper drugs earlier to achieve one more patient with improvement in attention. Analyses will be considered statistically significant if $95 \%$ confidence intervals for group differences do not include 0 , compatible with a 2-sided $p<0.05$. For chances of eventual seizure freedom and "seizure and AED freedom"; we will calculate a relative risk and a risk difference with $95 \%$ confidence intervals. Chances of eventual seizure freedom and "seizure and AED freedom" will be compared between the groups at 24 months after surgery and at 20 months following start of AED withdrawal; for the early withdrawal arm this will be at 24 months and for the late withdrawal arm at 32 months.

\section{Sample size calculation}

The study is powered based on the primary neuropsychological measures attention and executive function, determined with the EpiTrack Junior. We plan to study a continuous response variable from a late withdrawal (control) versus an early withdrawal (experimental) group with one control per experimental subject. For the sample size calculation, we used the reference values given in the Introduction and first validation of EpiTrack Junior (C Helmstaedter et al. 2010), which presents reference values of healthy children and children with epilepsy. The mean (SD) EpiTrack Junior score in healthy children was 32.6 (2.40). In children with epilepsy the mean (SD) score was 29.5 (4.70). For sample size calculations, we calculated the sample size using the SD of the epilepsy patients. Based on numbers of patients operated on per year in the participating medical centers and excluding patients with incompletely resected lesions, multifocal MRI abnormalities, an epileptic EEG postoperatively and use of a maximum of three AEDs at time of surgery (the numbers derived from the previous retrospective study (Boshuisen et al. 2012)), we estimated to expect to include at least 90 patients per year. By including at least 150 patients in the trial, with 75 patients per arm, we will be able to detect a true difference in the mean response of 2.16 points in EpiTrack Junior score (which corresponds to a difference of $0.46 \mathrm{SD}$ ) with a 2-sided alpha $=0.05$ and power (1-beta) $=0.8$. This difference is considered clinically relevant, as for the EpiTrack a 0.5 SD difference, can reflect a shift towards a milder impaired group of epilepsy patients (C Helmstaedter et al. 2010). One interim analysis according to O'Brien Fleming is planned after half of the planned number of patients has reached their endpoints. For reasons of possible dropout, we will aim to include 180 patients. 


\section{Ethical approval}

The multicenter trial will start in the University Medical Center Utrecht, the Netherlands, in November 2015. Ethical permission has been obtained from the ethical committee of the University Medical Center Utrecht, which is the leading ethical committee. Eight sites: University Hospitals of Lyon ( $\mathrm{HCL})$, France; University Hospital Strasbourg, France; Epilepsy Center Freiburg, Epilepsy Center Kork, University Hospital Heidelberg, Germany; Great Ormond Street Hospital for Children NHS Foundation Trust, London, and Young Epilepsy, Lingfield, United Kingdom; Western General Hospital Edinburgh, Scotland; Hôpitaux Universitaire de Genève, Switzerland, will join the trial shortly thereafter. Other centers, that have adequate experience with pediatric epilepsy surgery, are welcome to participate.

\section{Safety monitoring}

To minimize the risk for the participants in the proposed trial, we will exclude patients with those characteristics that have previously been shown to predict unfavorable outcome (Boshuisen et al. 2012). In our retrospective cohort we simulated the prospective randomized trial on 478 patients who would be eligible based on their exact inclusion and exclusion criteria. In this group, the interval to start of AED reduction was not associated with seizure recurrences during or after $A E D$ withdrawal ( $p=0.155, \mathrm{HR} 0.94 ; \mathrm{Cl} 0.86-1.02$ per 3 months), nor with eventual seizure freedom ( $p=0.551, \mathrm{HR} 0.96 ; \mathrm{Cl} 0.85-1.09$ ) or seizure and AED freedom ( $p=0.516, \mathrm{HR} 1.01 \mathrm{Cl} 0.97-1.06$, Cox regression analysis). Based on these analyses, we expect an overall comparable percentage of seizure recurrences in both treatments arms, similar to the number or recurrences encountered in everyday clinical practice when starting tapering of AEDs.

We appointed a Data Safety Monitoring Board (DSMB) that will analyze our data to warrant safety and to monitor inclusion rates and assess inclusion feasibility. At 24 months a blinded interim-analysis will be performed by the DSMB to investigate superiority of one of the treatment arms with regard to the primary outcome measure. If the difference in attention between groups is more than $1 \mathrm{SD}$ (age appropriate average reaction time), the DSMB may advise to prematurely stop the trial, if early withdrawal shows to be superior, the remaining patients in the late withdrawal group who still await start of AED reduction are allowed to withdraw medication from that time on. A test result with a 2 -sided $p<0.0054$ will be declared significant.

With regard to safety; due to the design of the trial, an interim safety analysis comparing both treatment arms at one moment is not possible. This is because we expect in both arms relapse risks to increase particularly once AED withdrawal is started. Thus, in the early withdrawal group recurrences probably occur sooner than in the late withdrawal group, although the total risk of relapse at the end of follow-up is not expected to differ between groups. This implies that 
at interim time points, when inherently more patients are under AED reduction (at increased risk of relapse) in the early arm, a fair comparison of relapse between both groups is not feasible. To deal with this, we will assess safety in each arm separately. The study intervention will be considered safe when seizure recurrences occur in less than $40 \%$ of both treatment arms. This percentage is chosen based on the general seizure freedom rate after childhood epilepsy surgery of 41 to 93 \% (Spencer and Huh 2008; Ryvlin, Cross, and Rheims 2014). Based on previous identification of risk factors for seizure recurrences, we expect recurrence rates in the patients included in the trial to be $<30 \%$, due to exclusion of patients with identified risk factors (Spencer and Huh 2008; Boshuisen et al. 2012; Ryvlin, Cucherat, and Rheims 2011; Cossu et al. 2008). We do not expect, however, the seizure status to be better than in normal clinical practice. Because of the expected cognitive benefit due to a longer AED-free period, a $40 \%$ rate of seizure relapse will still be accepted. The DSMB statistician will analyze the number of patients with seizure recurrences every 3 months during trial duration, in order to allow early awareness of unexpected high numbers of recurrences, exceeding the $40 \%$ limit. In this sequential testing safety model (Fig. 2), a 1-sided $p$ value $<0.05$ will be considered significant for the safety monitoring (Whitehead 1997; MPS Research Unit 2000)"

\section{Adverse event reporting}

For this study, recurrent epileptic seizures during AED withdrawal that need hospitalization do not necessarily have to be recorded as serious adverse events (SAEs), but are noted in the case report form (CRF) as one of the outcome measures, and will be included in the interim safety analyses by the DSMB. Epileptic seizures that lead to a potentially life-threatening situation, such as status epilepticus, will be reported as SAEs. All SAEs will be reported through the web portal "ToetsingOnline," to the accredited ethical committee that approved the protocol, within 15 days after the sponsor has first knowledge of the serious adverse reactions. Participating centers will inform the sponsor about the SAEs. Reporting of SAEs that result in death or are life-threatening should be expedited. The expedited reporting will occur not later than 7 days after the responsible investigator has first knowledge of the adverse reaction. This is for a preliminary report with another 8 days for completion of the report. 


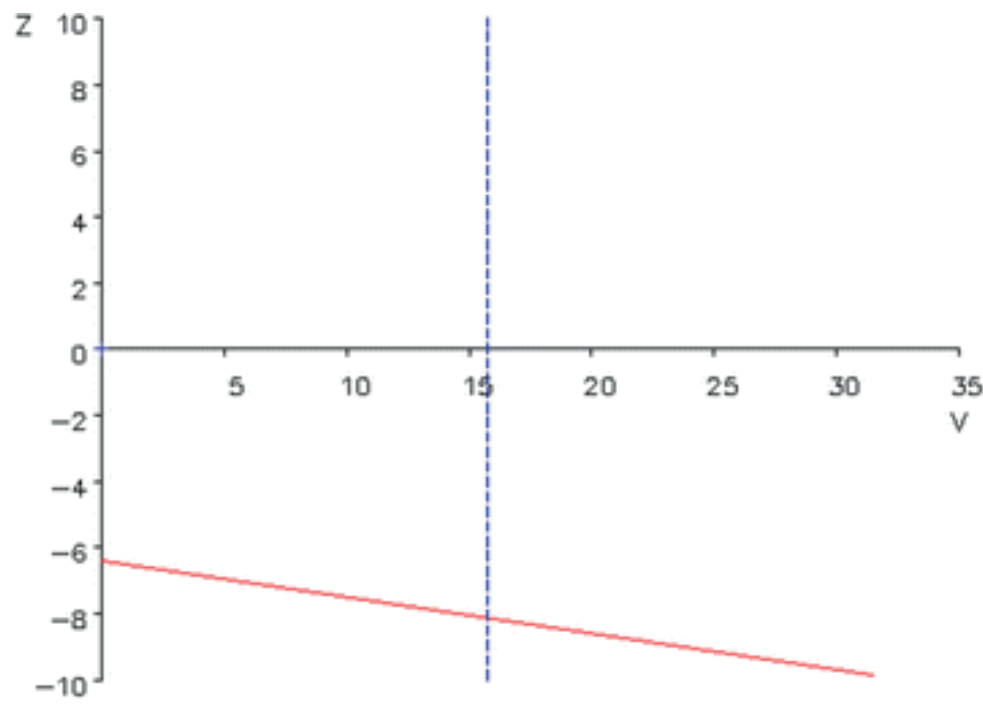

Figure $\mathbf{2}$ | Sequential testing safety profile. The plot is based on a likely sample size of 75 patients per treatment strategy, a 1 -sided $a=0.05$, an expected seizure recurrence rate $\leq 30 \%$ and a cut-off point for safety, which is a recurrence rate of $\geq 40 \%$. The red boundary represents the safety margin. The following formulas will be used to plot data in the graph: $Z=-(F-n \times P c)(Z=$ number of observed seizure recurrences - number of expected seizure recurrences), $V=n \times P c$ $(1-P c)(V$ is the variance of $Z$ and stands for the amount of information in $n$ patients, with $\mathrm{n}$ being the number of included patients, Pc the expected seizure recurrence risk (i.e., for the selected patient cohort: 0.30 ) and $F$ the number of observed seizure recurrences). The vertical line is the anticipated value for $V$ for a sample size of 75 patients. 


\section{Discussion}

We present the protocol of a European pragmatic randomized clinical trial, designed to investigate the possible benefits and the safety of early AED withdrawal after epilepsy surgery in children. We hypothesize that early AED withdrawal is safe, in terms of seizure relapse rates and eventual seizure outcomes, and will improve cognitive functioning, behavior and quality of life, compared to late withdrawal. We will investigate these hypotheses by randomly allocating patients to an early withdrawal group that starts withdrawal of medication 4 months after surgery, versus a late withdrawal group, that starts withdrawal of medication after 12 months. Justification of this trial needs to be discussed. The non-experimental evidence obtained so far on safety of AED withdrawal in relation to seizure outcome seems strong and consistent (Boshuisen et al. 2012; Yardi et al. 2014). In analyses that had been elaborately adjusted for confounding, there was a slightly increased recurrence risk for earlier withdrawal, but not for regain of seizure freedom rates and eventual seizure outcome. Moreover, findings on cognitive outcome after AED withdrawal all pointed towards cognitive improvement on several domains (Shields 2000; Cross 2002; Van Schooneveld and Braun 2013; Loring and Meador 2004; Meador 2002; Lossius et al. 2008; Skirrow et al. 2011; Hessen et al. 2006; AP Aldenkamp et al. 1993, 1998; Van Schooneveld et al. 2013; Hallböök et al. 2013; Meekes et al. 2013). Why then do we think that the trial will have important added value? Through randomized allocation the study population will be broader than in the non-experimental studies which only pertained to patients for whom AED withdrawal had already been decided about. Consequently, results will have wider clinical applicability and will apply to all patients who are eligible for withdrawal decision- making. A very important asset will be obtaining baseline comparability of treatment arms for both known and measurable confounders, but also for unknown or unmeasurable confounders. In principle, the non-experimental evidence, although elaborately adjusted for known confounders, does leave residual confounding as an explanation. The trial will allow for full follow-up of all children randomized and for intention-to-treat analysis. Finally, through the above, stronger evidence will contribute to our ultimate goal, implementation of evidence-based practice concerning AED withdrawal. Although previous non-experimental research does seem to indicate benefits of early AED withdrawal, it is especially the remaining uncertainty about residual confounding that leads us to claim that there currently is genuine equipoise about timing of withdrawal and, therefore, that random allocation is ethically justified. This issue will have to be addressed with regard to trial feasibility. Throughout European centers participating in the trial, there are regional preferences concerning AED withdrawal timing, and non-experimental evidence has already led to changes in clinical practice concerning withdrawal timing. It will be vital for successful completion of the trial that participating physicians can and will be convinced that there still is true equipoise, as a sufficient basis for studying randomized AED withdrawal timing to the ultimate benefit of children who have undergone epilepsy surgery. 
Recruitment of participants for this study might be challenging for three reasons. First, in some of the participating countries and centers, AED withdrawal policies are still rather conservative, conflicting with the proposed early AED strategy. This may either be due to reluctance of parents or patients to risk a seizure relapse, now that seizure freedom has finally been achieved following epilepsy surgery, or to fear of the treating neurologist or neurosurgeon that seizure control is permanently lost after a post-withdrawal relapse. In addition, in some patients treating physicians may require a postoperative MRI to prove complete resection of the epileptogenic lesion before considering AED withdrawal. Planning of MRI scanning before randomization may not always be feasible in the proposed short time period. Second, in many participating centers, AED withdrawal policies already tend to change towards earlier reduction, as a consequence of the findings from the previous retrospective observational TTS study (Boshuisen et al. 2012). These changing opinions on AED withdrawal policies are not only known among physicians, there is also an increasing awareness of safety of early withdrawal among parents of children who have been operated on. Therefore, willingness to be randomized to the late withdrawal strategy arm may prove difficult in these centers. Third, some treating physicians may not want to withdraw AEDs within a set maximum time frame of 8 months, or only consider reduction of medication instead of complete discontinuation of AEDs, fearing that relatively early, rapid or complete withdrawal may increase the risk of seizure relapse. To prevent limited recruitment of patients we will encourage participating physicians to counsel parents and patients optimally, based on the available evidence regarding safety of AED withdrawal, which can be summarized as follows: although retrospective data suggest that early withdrawal is safe and does not compromise eventual seizure outcome or treatability of seizure relapse, definite proof of safety in a predefined population of children who underwent anticipated curative surgery is still required, justifying randomization to either treatment arm. Furthermore, although we anticipate cognitive improvement following AED discontinuation, the - possibly enduring - cognitive advantages of early over late withdrawal need to established, which is important to enable careful balancing of risks and benefits of early AED discontinuation in individual patients in the future.

\section{Trial organization}

Steering committee

The steering committee carries the ultimate responsibility for the trial. Specific tasks of the steering committee are:

1. Design and final approval of the study protocol

2. Approval of the amendments to the study protocol

3. Deciding whether or not to continue the trial based on the recommendations of the DSMB

4. Approval of manuscripts and publications of the trial 
The Trial committee is constituted of the principle investigator of each participating center and of the members of the Steering committee.

As of 16 March 2015, the members of the Steering Committee are (in alphabetical order): A. Arzimanoglou, child neurologist, University Hospitals of Lyon, France, co-principle investigator; K. Boshuisen, research physician, University Medical Center Utrecht, the Netherlands; K.P.J Braun, child neurologist, University Medical Center Utrecht, the Netherlands, co-principle investigator; J.H. Cross, child neurologist, Great Ormond Street Hospital for Children NHS Foundation Trust, London, and Young Epilepsy, Lingfield, United Kingdom, co-principle investigator; K. Geleijns, child neurologist University Medical Center Utrecht, the Netherlands, coordinating investigator; H.J. Lamberink, PhD student, University Medical Center Utrecht, the Netherlands; M.M.J. Schooneveld, child neuropsychologist, University Medical Center Utrecht, the Netherlands; C.S.P.M Uiterwaal, epidemiologist and statistician, University Medical Center Utrecht, the Netherlands.

Principle and coordinating investigators (other than those listed in the steering committee) at each site are in alphabetical order: R. Chin, child neurologist, Western General Hospital Edinburgh, Scotland; T. Polster, child neurologist, Krankenhaus Mara, Epilepsiezentrum Bethel, Bielefeld, Germany; G. Ramantani, child neurologist, Epilepsy Center Freiburg, Epilepsy Center Kork, University Hospital Heidelberg, Germany; A. De Saint-Martin, child neurologist, University Hospital Strasbourg, France; M. Seeck, epileptologist, Hôpitaux Universitaire de Genève.

\section{Data Safety Monitoring Board}

The DMSC analyses the unblinded data on a permanent basis and formulates recommendations for the Steering Committee on the continuation of the trial. The Data Monitoring Committee may also offer unsolicited recommendations. Members of the Data Monitoring Committee are: Prof. Dr. Oebo Brouwer, child neurologist, epileptologist, UMCG; Dr. Ingeborg van der Tweel, statistician, UMCU; Prof. Dr. Jaap Kappelle, neurologist, UMCU.

\section{Trial status ${ }^{3}$}

Ethical approval has been obtained for the University Medical Center Utrecht. The other participating centers are in the process of obtaining approval. No patients have yet been included in the trial.

Abbreviations

AED: antiepileptic drug

CBCL: Child Behavior Checklist

$\mathrm{Cl}$ : confidence interval

CRF: case report form

DSMB: Data Safety Monitoring Board 
EEG: electroencephalogram

HASES: The Hague Side Effects Scale

HR: hazard ratio

IQ: Intelligence Quotient

MRI: magnetic resonance imaging

PedsQLTM: Paediatric Quality of Life InventoryTM

SD: standard deviation

PIQ: Performal IQ

SAE: severe adverse event

SD: standard deviation

TTS: TimeToStop

VIQ: Verbal IQ

\section{Acknowledgments}

We thank Eltje Bloemen-Carlier, who supported in the design of the trial and will support the conduct of the trial; we thank the Dutch Epilepsy Fund for funding of the TimeToStop project. 







\title{
Why the TimeToStop trial failed to recruit: a survey on antiepileptic drug withdrawal after paediatric epilepsy surgery
}

\author{
Lamberink HJ \\ Geleijns K \\ Otte WM \\ Arzimanoglou $\mathrm{A}$ \\ Cross JH \\ Korff CM \\ Ramantani G \\ Braun KPJ
}

Epileptic Disorders. 2018;20(5):374-385 


\section{Abstract}

Following the results of the multicentre European retrospective "TimeToStop" cohort study, we initiated a randomised trial to determine cognitive benefits of early postoperative antiepileptic drug withdrawal. Unfortunately, the trial failed to recruit and was terminated, as almost all parents preferred early drug withdrawal. The objectives of the current survey were to obtain insight into current practices regarding drug withdrawal after paediatric epilepsy surgery among epileptologists, and better understand the reasons for difficulties in recruitment. A survey was sent to three international epilepsy surgery networks, questioning drug withdrawal policies. Forty-seven (19\%) surveys were returned. For polytherapy, withdrawal was started at a median of three and six months by the TimeToStop collaborators and other paediatric epileptologists, respectively. Withdrawal was completed at a median of 12 and 20 months, respectively. For monotherapy, tapering was initiated at five and 11 months in these two groups, and ended at a median of seven and 12 months, respectively. Most TimeToStop collaborators believed that it was not justified to wait 12 months after surgery before reducing AEDs, regardless of the number of AEDs taken. Current AED policies in Europe have changed as a consequence of the retrospective TimeToStop results, and this accounts for why recruitment in a randomised trial was not feasible. 
On achieving seizure freedom following epilepsy surgery, the ultimate proof of surgical success and thus of "cure" is the complete discontinuation of antiepileptic drugs (AEDs). In children, AED withdrawal favours, on average, improved psychomotor speed and intelligence (Skirrow et al. 2011; Van Schooneveld et al. 2013; Boshuisen, Van Schooneveld, et al. 2015). A multicentre European retrospective cohort study strongly suggested that the timing of postoperative AED withdrawal does not influence eventual seizure outcomes (Boshuisen et al. 2012); although the risk of seizure recurrence was increased with earlier AED withdrawal, there was no association with long-term freedom of seizures or medication status at final follow-up visits. Early withdrawal therefore uncovers incomplete surgical success sooner, while preventing overtreatment for the large majority of children in whom surgery has successfully removed the epileptogenic zone. To assess the potential cognitive benefits of early versus late withdrawal, we initiated the "TimeToStop" randomised controlled trial (EudraCT number 2011-005971-18) (Boshuisen, Lamberink, et al. 2015). We aimed to compare cognitive functioning, intelligence, and seizure outcome at 12 and 24 months after epilepsy surgery between children who were randomised to start withdrawal at four months and those who started withdrawal at 12 months after surgery. Eight centres in five countries agreed to participate. In November 2015, recruitment was started in Utrecht, the Netherlands, and was in preparation in the other centres. Up to February 2017, 47 children were screened, of whom 35 were not eligible (supplementary table 1). Of the 12 children who were eligible, their parents declined participation; $11 \mathrm{did}$ not want to wait until 12 months after surgery before withdrawing medication, and the parents of one child considered withdrawal at four months too early. None of the parents or children agreed with participation based on randomisation to determine the timing of postoperative AED withdrawal. After deliberation with the trial collaborators, it was decided to terminate recruitment for reasons of non-feasibility.

To gain insight into current practices and better understand the reasons for difficult recruitment in the TimeToStop trial, we aimed to describe AED withdrawal policies among paediatric epileptologists using a short survey. The survey was based on the following hypotheses:

- current beliefs about safety and benefits of early postoperative AED withdrawal among treating physicians justify premature discontinuation of the TTS trial for feasibility issues;

- $\quad$ partners of the original TTS study group and European paediatric epileptologists tend to withdraw medication sooner than others;

- the previous retrospective TTS cohort study has changed decision-making regarding AED withdrawal. 


\section{Methods}

A survey was created focusing on paediatric neurologists, but also enabling neurologists or physicians who treat both children and adults, to respond. The full survey can be found in Appendix 1 and contains several items on the timing of postoperative AED withdrawal in children who underwent anticipated curative epilepsy surgery, factors influencing timing, and personal preferences of participants and parents/children with epilepsy. The survey was widely distributed among epilepsy surgery specialists collaborating in three broad networks: (1) the U-Task (European Task Force for Epilepsy Surgery in Children), which meets twice a year to discuss surgical cases and collaborative research projects; (2) the E-PILEPSY consortium, an EUfunded pilot reference network of epilepsy surgery centres, aiming to improve access to, and outcome of, epilepsy surgery and harmonise (pre-)surgical approaches across Europe; and (3) the mailing list of the International League Against Epilepsy (ILAE) Pediatric Epilepsy Surgery Task Force. The mailing lists contained paediatric and adult neurologists, neurosurgeons, and other staff involved, with considerable overlap between the three lists.

When information on the timing of AED withdrawal was given as a range, the average was used as input for the analysis. The values were non-normally distributed, hence summary statistics are given as medians and interquartile ranges (IQR), and a Mann-Whitney $U$ test was used to test group differences. Results were compared between respondents who collaborated in the retrospective TTS study or prospective TTS trial and all other participants, and between European (TTS collaborators and non-collaborators) and non-European respondents.

\section{Results}

The survey was sent to 251 addresses; 47 (19\%) surveys were returned by 32 paediatric epileptologists and 15 specialists who treated both children and adults. Nine respondents had participated in the previous retrospective TimeToStop study (Boshuisen et al. 2012)or the prospective TimeToStop trial (Boshuisen, Lamberink, et al. 2015) described in the introduction. There were 38 additional respondents, from Europe (22), Brazil (2), India (2), Japan (5), Mexico (1), South Africa (1), Thailand (1), and the United States of America (4). The number of unique centres was 39 from 21 countries.

A comparison of the postoperative timing of AED withdrawal between TTS collaborators and other respondents is given in figure 1 and table 1 . With regards to both initiating and completely discontinuing AEDs, this was earlier for TTS collaborators than the other respondents. For children on polytherapy, the median start of withdrawal was three months following surgery for the TTS collaborators compared to six months for other respondents $(U=258.5 ; p=0.02)$. AEDs were completely tapered off after a median of 12 months compared to 20 months, respectively $(U=246 ; p=0.002)$. For children on monotherapy at the time of surgery, AEDs were reduced at 
a median of five months following surgery for the TTS collaborators compared to 11 months for other respondents $(U=222.5 ; p=0.08)$, and completely discontinued at seven months compared to 12 months, respectively $(U=243 ; p=0.01)$. European respondents started and discontinued AEDs earlier compared to non-Europeans in the case of monotherapy (starting at a median of six vs. 12 months, and discontinuation at nine vs. 17 months, respectively); for polytherapy, the two groups started AED withdrawal at a similar time (a median of five vs. six months, respectively), but there were differences in the timing of complete discontinuation (16 vs. 24 months, respectively).

TTS $(n=9)$ 直 other $(n=38)$


Figure 1 | The timing of antiepileptic drug (AED) withdrawal after paediatric epilepsy surgery in the case of polytherapy and monotherapy, compared between the two cohorts of TTS collaborators and other respondents. The boxes show the median and interquartile range (IQR), and the whiskers extend to $1.5^{*} \mathrm{IQR}$. End: complete discontinuation of last AED; start: start of AED withdrawal; TTS: collaborators of the TimeToStop study on safety of early drug tapering and/or the TimeToStop trial on the cognitive benefits of early drug tapering. A summary of the statistics is provided in table 1. 
Table 1 | Median time corresponding to initiation of AED withdrawal and complete discontinuation compared between the three groups.

\begin{tabular}{llll}
\hline & & Start of AED withdrawal & $\begin{array}{l}\text { Complete discontinuation of } \\
\text { last AED }\end{array}$ \\
\hline Polytherapy & TTS & $3(1-4)$ & $12(9-12)$ \\
& Other & $6(3-6)$ & $20(13-24)$ \\
& Mann-Whitney U: & $258.5, p=0.02$ & $246, p=0.002$ \\
Monotherapy & $5(4-6)$ & $7(6-11)$ \\
& TTS & $11(6-13)$ & $12(9-18)$ \\
& Other & $222.5, p=0.08$ & $243, p=0.01$ \\
\hline
\end{tabular}

All given values are medians (IQR) corresponding to months after paediatric epilepsy surgery (rounded to full months). Data are presented graphically in figure 1.

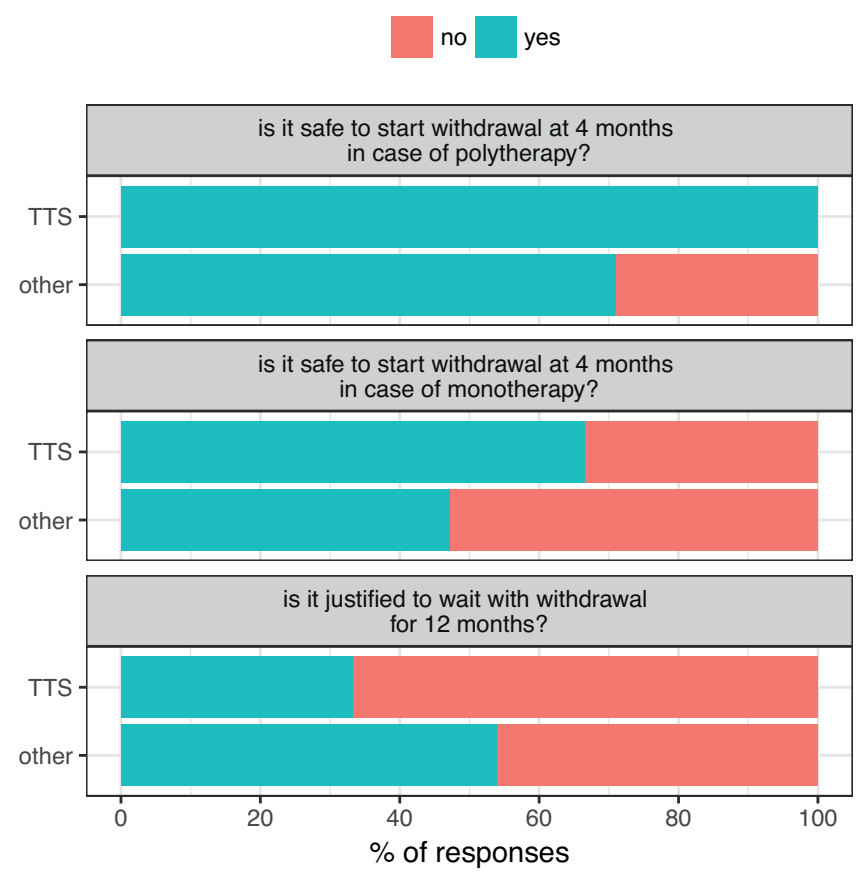

Figure 2 | Responses to the three questions, compared between TimeToStop (TTS) collaborators and all other respondents. 
Three questions were asked regarding the safety and justification of starting withdrawal at either four or 12 months after surgery; the responses are summarised in figure 2 . For polytherapy, all TTS collaborators deemed initiation of AED withdrawal safe at four months, compared to $71 \%$ of other respondents. For monotherapy, $67 \%$ of TTS collaborators deemed AED withdrawal safe at four months, compared to $47 \%$ of other respondents. Only $33 \%$ of TTS collaborators believed it was justified to wait until 12 months after paediatric epilepsy surgery, compared to $54 \%$ of others.

Figure 3 illustrates how respondents judged the different clinical factors that may influence AED withdrawal timing. Overall, the decision to completely wean off medication is taken more cautiously than the decision to start reduction of AEDs. The strongest reasons for not completely discontinuing medication were (as outlined in figure 3): incomplete resection of the epileptogenic zone (66\% of respondents), incomplete resection of the anatomical lesion (43\%), preoperative multifocal MRI abnormalities (33\%), and postoperative epileptic EEG abnormalities (30\%).

The combined strongest reasons to start AED withdrawal later or not at all were: incomplete resection of the epileptogenic zone (89\%) or anatomical lesion (85\%), postoperative EEG abnormalities (77\%), multifocal MRI abnormalities (74\%), neurocutaneous aetiology (63\%), previous epilepsy surgery (60\%), and depression as co-indication for AED treatment (60\%). Taper duration ranged from 0.5 to 18 months per drug, with a median of 3.0 months (IQR: 2.5-9).

The median (IQR) taper duration for TTS collaborators and other paediatric epileptologists was 2.5 months/drug (2.5-2.6) and 5.3 months/drug (3.0-9.8), respectively. Drugs with a longer taper period for some of the respondents were phenobarbital (17/37 responses), benzodiazepines (14/37), and carbamazepine (7/37) (supplementary table 2). Some respondents reported side effects experienced by patients as a reason for initiating withdrawal earlier, especially when these were more prominent after successful epilepsy surgery.

Fifteen physicians were treating both children and adults, which allowed for a comparison of withdrawal practices. On average, physicians were more careful regarding early withdrawal for their adult patients, and started withdrawal later for both polytherapy (median time difference: two months; IQR: 0-5) and monotherapy (median time difference: two months; IQR: 0-9). Complete discontinuation was also later in adult cases, with a median time difference of six months (IQR: 0-24) and 12 months (IQR: 0-12) in the case of polytherapy and monotherapy, respectively. For adults, 50\% deemed initiation of AED withdrawal safe at four months in the case of polytherapy and $14 \%$ in the case of monotherapy, compared to $53 \%$ and $46 \%$ for children, respectively. Sixty percent stated that it was justified to wait for 12 months after adult epilepsy surgery, compared to $47 \%$ in paediatric care. 
Of all the respondents, $32(68 \%)$ indicated that they would advise mainly in favour of withdrawal, four (9\%) were in favour of counselling towards continuation of medication, and $11(23 \%)$ indicated that counselling depends on case-specific factors. This is reflected by the impression of parental preferences in the respondents' centres: 30/45 (67\%) indicated that parents would prefer early withdrawal and $5 / 45(11 \%)$ late withdrawal. For $7 / 45(16 \%)$, this depended on the situation, and for $3 / 45(7 \%)$ of respondents, most children and parents would prefer to reduce the dose but not completely discontinue all AEDs.

The last question addressed whether results from the previous retrospective TimeToStop study (Boshuisen et al. 2012) influenced clinical practice. A total of 32/46 (70\%) indicated that this was the case; $88 \%$ of all TTS epileptologists (one indicated that the results did not influence his/her clinical practice), compared to $66 \%$ of the other paediatric epileptologists. Most responded stating that they now consider AED withdrawal earlier as a result of this study. As one respondent put it: "no more waiting for the magic two years".

\section{Discussion}

This study shows that European paediatric epileptologists who participated in the TTS study started tapering off AEDs, on average, between three and five months (in the case of polytherapy or monotherapy, respectively) after successful epilepsy surgery, which is earlier than the median of 6-11 months for the other respondents. In addition, the vast majority of the TTS respondents were unable to justify waiting for 12 months at this moment, and half of the other respondents shared this opinion. In addition, the majority of respondents indicated that the retrospective TTS study influenced their clinical decision-making towards earlier postoperative AED withdrawal. These results, together with the experiences of the coordinating centre of the planned TTS trial (UMC Utrecht), where all parents of eligible patients refused randomisation, was the rationale for prematurely stopping the trial without having included a single patient.

A comparison of our data with previous surveys on postoperative medication policy is problematic for several reasons. The three surveys that were performed in 2007 (Berg et al. 2007), 2012 (Téllez-zenteno et al. 2012), and 2013 (Swisher and Sinha 2013) were all performed in the US and Canada. Furthermore, adult neurologists were only questioned in one survey (Berg et al. 2007), and the other two involved mixed answers from paediatric and adult neurologists (Téllez-zenteno et al. 2012; Swisher and Sinha 2013). We have shown that when an epileptologist treats both children and adults, the timing of AED withdrawal is later for adults, especially regarding the timing of complete discontinuation. This may be related to the potential consequences regarding, for example, employment and driving. The median time to first reduction was 12.5 months in the European TimeToStop study (Boshuisen et al. 2012), 
AEDs are reduced/discontinued: as usual later not at all

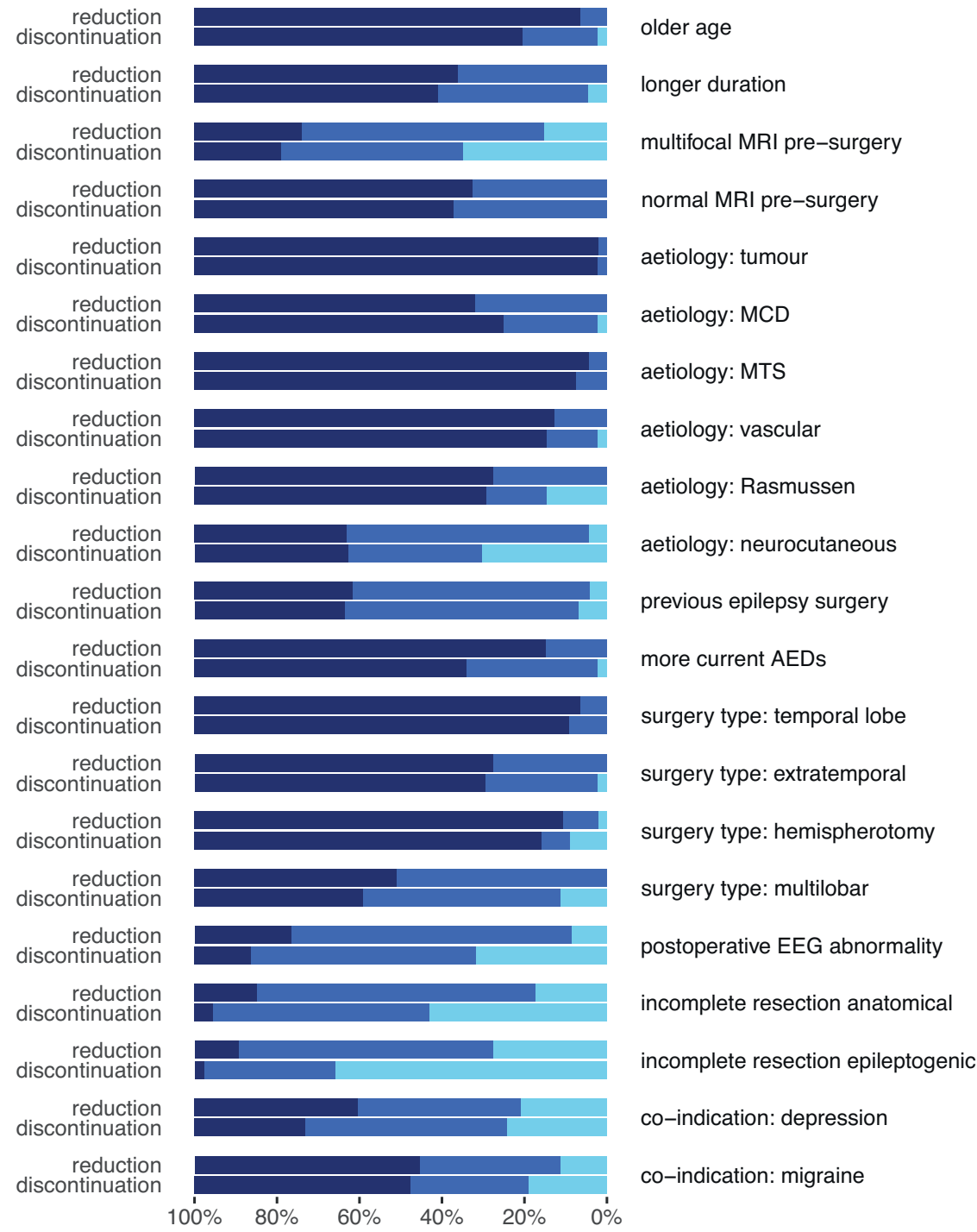

Figure 3 | Factors influencing the decision to start reduction of AEDs and complete discontinuation of AEDs. The data are based on answers to the question: "In the presence of this factor, would you (reduce or discontinue) AEDs (a) as usual, (b) later, or (c) not at all!". 
which included 766 children who were operated on between 2000 and 2008 (polytherapy and monotherapy combined). Relative to this study, and in line with our own experience, the current results show a marked shift to earlier AED withdrawal after paediatric epilepsy surgery. This survey illustrates current practices, however, there are several limitations. First, the total number of responses is low making strong generalizations invalid. However, the response rate of $19 \%$ is misleadingly low because it was not possible to provide an informative response rate in this study; the email lists that were used as a basis for the survey also contained those of neurosurgeons and other experts who may not have been directly involved in decisions regarding medication. Also, several adult neurologists received the survey and may have ignored it because it focused on a paediatric population. Since the survey was directed at the caring physician and not at the patient, we have no direct information on the preferences of the patient. As for the average timing of postoperative AED withdrawal, the indicated numbers are only averages across both low- and high-risk cases. Many respondents indicated a range for the timing, for example, starting AED withdrawal in the case of polytherapy 3-12 months after surgery. In this case, for the ideal candidate, AEDs would undoubtedly be tapered off at three months. However, in this study, the mean of the range, i.e. 7.5 months, would have been considered for analysis, which might influence the average. Nevertheless, we can conclude that the median time for starting AED withdrawal is well below 12 months after anticipated successful epilepsy surgery for the majority of European paediatric epileptologists. Conclusions regarding specialists outside Europe cannot be made because of the significant heterogeneity between countries. We can only speculate on the reasons for a more conservative AED policy outside Europe. If the fear of poor seizure outcome after early withdrawal persists among paediatric epileptologists, future comparative studies might be designed to address the safety of early withdrawal in specific populations, particularly children with higher-risk profiles. 
Supplementary Table 1 | Eligibility for the TimeToStop trial and reasons for non-eligibility.

\begin{tabular}{ll}
\hline Eligibility & Number of children \\
\hline Eligible for inclusion & 12 \\
Objection against late withdrawal & 11 \\
Objection against early withdrawal & 0 \\
Willing to participate & 35 \\
Not eligible & 8 \\
Age at surgery > 15 years & 10 \\
Age at surgery <6 years (too young to perform Epitrack Junior) & 8 \\
No Epitrack Junior performed (not able to take it or not performed) & 3 \\
Postoperative epileptiform discharges on EEG or ECoG & 1 \\
No postoperative seizure-freedom & 1 \\
Vagus nerve stimulator & 2 \\
Not using AEDs before surgery & 1 \\
Only surgery in our centre, follow-up elsewhere & 1 \\
Already started AED withdrawal <4 m because of side-effects & 1 \\
\hline
\end{tabular}

Total number of patients who were screened and approached for participation in the TimeToStop trial, between 1-11-2015 until 28-2-2017. 
Supplementary Table 2 | Does the type of drug influence timing of AED withdrawal?

\begin{tabular}{llll}
\hline Drug name & $\begin{array}{l}\text { Longer taper } \\
\text { period }\end{array}$ & $\begin{array}{l}\text { Later start } \\
\text { withdrawal }\end{array}$ & $\begin{array}{l}\text { No complete } \\
\text { discontinuation }\end{array}$ \\
\hline phenobarbital & 17 & 1 & 0 \\
benzodiazepines & 14 & 3 & 0 \\
carbamazepine & 7 & 5 & 0 \\
phenytoin & 4 & 0 & 0 \\
vigabatrin & 2 & 2 & 0 \\
valproic acid & 2 & 1 & 0 \\
primidone & 2 & 1 & 0 \\
oxcarbazepine & 2 & 1 & 0 \\
topiramate & 2 & 0 & 0 \\
lamotrigine & 1 & 2 & 0 \\
bromide & 1 & 0 & 0 \\
zonisamide & 1 & 0 & 0 \\
levetiracetam & 0 & 3 & 0 \\
lacosemide & 0 & 1 & 0 \\
cannabidiol & 0 & 0 & 1 \\
\hline lat & 2 & 2 & 0 \\
\hline
\end{tabular}

$37 / 47$ answered the question. Six respondents specifically mentioned that no drug influenced the decision. No drug was indicated to be related to not starting AED withdrawal. 


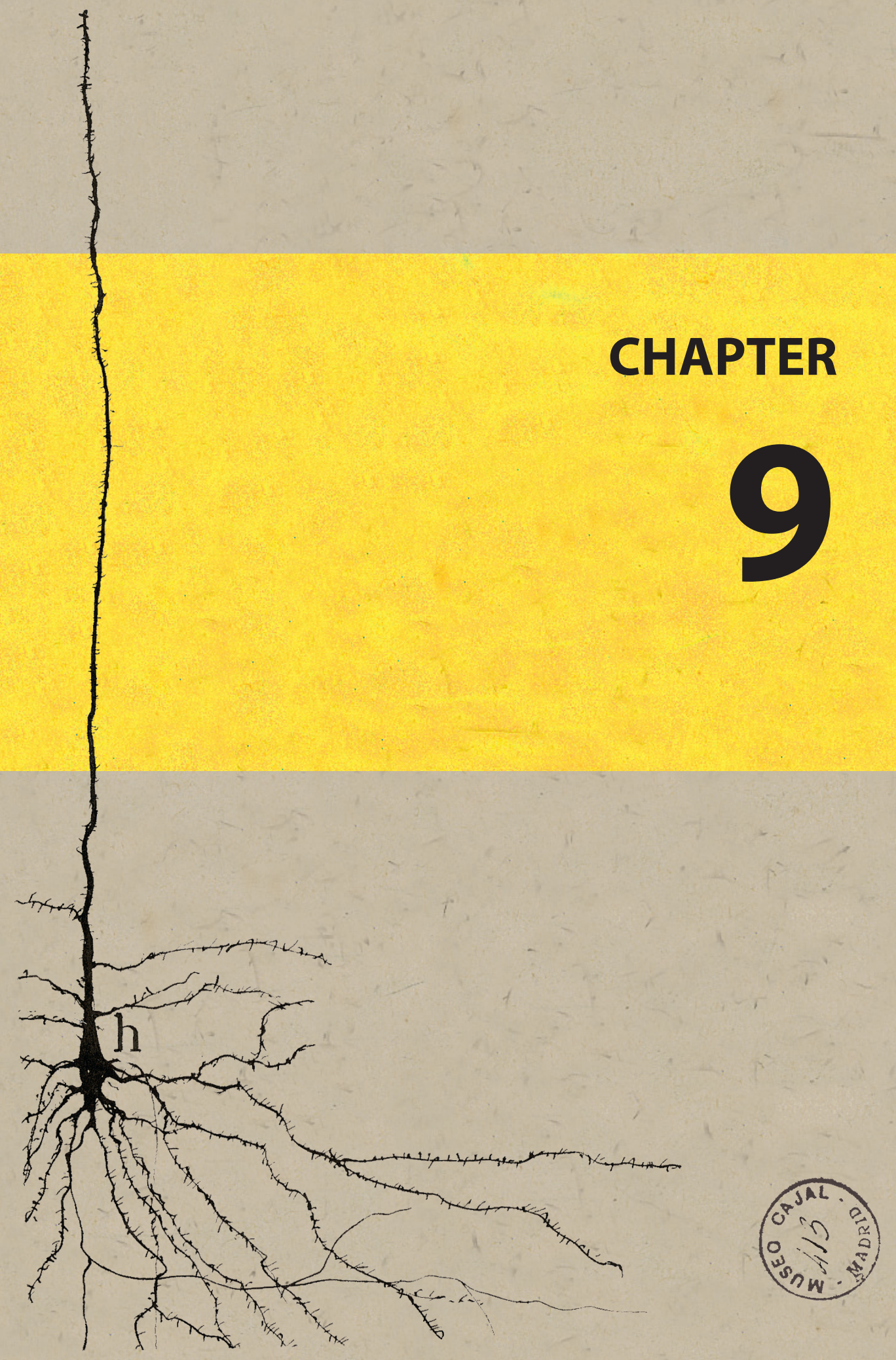




\section{Individualized prediction of seizure relapse and outcomes following antiepileptic drug withdrawal after pediatric epilepsy surgery}

Lamberink HJ

Boshuisen $\mathrm{K}$ Otte WM Geleijns K Braun KPJ

TimeToStop Study Group Epilepsia. 2018;59(3):e28-e33 


\section{Abstract}

The objective of this study was to create a clinically useful tool for individualized prediction of seizure outcomes following antiepileptic drug withdrawal after pediatric epilepsy surgery. We used data from the European retrospective TimeToStop study, which included 766 children from 15 centers, to perform a proportional hazard regression analysis. The 2 outcome measures were seizure recurrence and seizure freedom in the last year of followup. Prognostic factors were identified through systematic review of the literature. The strongest predictors for each outcome were selected through backward selection, after which nomograms were created. The final models included 3 to 5 factors per model. Discrimination in terms of adjusted concordance statistic was 0.68 (95\% confidence interval [CI] 0.670.69) for predicting seizure recurrence and 0.73 (95\% Cl 0.72-0.75) for predicting eventual seizure freedom. An online prediction tool is provided on www.epilepsypredictiontools. info/ttswithdrawal. The presented models can improve counseling of patients and parents regarding postoperative antiepileptic drug policies, by estimating individualized risks of seizure recurrence and eventual outcome. 


\section{Introduction}

After successful pediatric epilepsy surgery, a pressing question for many patients and their caretakers is whether and when to reduce antiepileptic drugs (AEDs). Being able to discontinue drugs is the ultimate proof of successful epilepsy surgery. Stopping medication will prevent further drug adverse effects and may improve cognitive domains, such as psychomotor speed, executive functions and IQ (Skirrow et al. 2011; Van Schooneveld et al. 2013; Boshuisen, Van Schooneveld, et al. 2015; Christoph Helmstaedter, Elger, and Witt 2016). The decision to withdraw AEDs is partially based on an assessment of the risk of seizure recurrence. Predictors of seizure recurrence on a group level have been identified in previous publications (Lee et al. 2008; Boshuisen et al. 2012; Park et al. 2010; Menon et al. 2012; Yardi et al. 2014; Berg et al. 2006) prognostic factors and proper time of AED withdrawal after surgery for medial temporal lobe epilepsy (MTLE, but prediction of relapse is not yet possible for an individual patient.

The TimeToStop study previously showed that early withdrawal unmasks surgical failure but is not related to long-term seizure outcomes (Boshuisen et al. 2012). This conclusion was based on multivariable survival analysis but did not yet enable individualized prediction of AED withdrawal outcomes. We now use the TimeToStop data to create nomograms and a web-based tool to enable estimation of individual risks, analogous to the recently developed prediction models for nonsurgical patients (Lamberink et al. 2017).

\section{Methods}

Data of 766 patients were available from the TimeToStop study; for an extensive description of data collection and definitions we refer to the earlier report (Boshuisen et al. 2012). In short, patients were identified retrospectively within 15 pediatric epilepsy surgery centers in Europe. Patients were included if they had epilepsy surgery between 2000 and 2008, were younger than 18 years at surgery, had at least 1 year of follow-up after surgery, and if postoperative AED withdrawal was initiated. Data were collected on clinical, surgical, and seizure outcome parameters.

Two outcome measures were used: first, seizure recurrence after start of AED withdrawal and second, the seizure status at final follow-up, with favorable outcome being defined as seizure freedom (including freedom from auras) for at least 1 year at final follow-up. We decided not to model for 'cure" (seizure and AED freedom at final follow-up), which was the third outcome measure in the original TimeToStop study, because the decision to completely discontinue medication is dependent on the policy of the individual physician, and these policies may change over time or may already have changed. A systematic review on risk factors of postoperative AED withdrawal previously identified 12 potential factors predictive of outcome (Lamberink, Otte, et al. 2015), listed in Supplementary Table 1. These factors were all available 
in the TimeToStop dataset.

Missing data were analyzed and imputed through multiple imputations using predictive mean matching. Heterogeneity between centers was tested, and when present, randomeffects modeling was used. Assumptions were tested: if the linearity assumption was violated, restricted cubic splines with 3 knots were used. Proportional hazard (Cox) regression analysis was used to account for different follow-up duration. For seizure recurrence, censoring time was the time to seizure recurrence. For seizure freedom, censoring time was time to seizure recurrence, only for those with unfavorable outcome. All patients with favorable outcome were censored at maximum follow-up. To create a useful set of predictors, the Akaike Information Criterion was used for backward selection of strongest predictors.

For the purpose of the prognostic models, variables with 3 or 4 items of which only 1 differed significantly from the reference category were dichotomized so that the nonsignificant categories were collapsed with the reference category. This was done for magnetic resonance imaging (MRI; multifocal MRI abnormalities vs focal MRI abnormality and normal MRI), for prewithdrawal electroencephalography (EEG; interictal epileptiform discharges vs no such discharges or no EEG performed), and for completeness of resection (incomplete resection of the anatomical lesion vs complete resection or completeness not determined). Within the nomograms, risk calculation of seizure recurrence was enabled for 2 and 5 years after start of AED withdrawal, and calculation of eventual seizure freedom was set at 6 years. We chose 6 years after start of AED withdrawal to model the chance of eventual "long-term" seizure freedom, creating a 1-year longer interval than the latest calculated 5-year relapse risk, as this intuitively allows for extra time to regain seizure freedom after restart of medication if a late recurrence has occurred.

Internal validation was accomplished by determining the concordance statistic for each model, corrected for overfitting using 200 bootstrap samples. Plotting of the predicted probabilities against observed proportions of the outcomes was done to visually assess calibration of the separate models. Internal-external cross-validation was performed by iteratively removing 1 of the 4 largest centers, refitting the model on the remaining data, and externally validating on the excluded dataset.

Analyses were performed with R Statistical software version 3.3.2, using the packages "MICE," "coxme," and "rms." Ethics approval was obtained for the original TimeToStop study. 


\section{Results}

One percent of data was missing and imputed (Supplementary Table 2). The seizure outcomes after AED withdrawal were the following: seizure recurrence in 95/766 patients (12\%) and seizure freedom in the last year of follow-up in 733/760 patients (96\%). Median followup after start of AED withdrawal was 39 months (interquartile range [IQR] 21-67 months) (Supplementary Table 3). Univariable relations between potential predictors and outcomes are shown in Supplementary Table 4.

One of the parameters, the presence of postoperative seizures (excluding seizures in the first 2 postoperative weeks), was not included in the analysis because none of the 13 cases experienced seizure recurrence, and consequently no parameters could be estimated. The median time to reduction (start tapering of the first drug) for these patients was 24 months (IQR 19-37), compared to 13 months (IQR 6-24) in the patients without postoperative seizures. AED status at final follow-up was known for 11 of these: 6 patients successfully discontinued AEDs and 5 were still using AEDs.

The strongest predictors were selected through backward selection, resulting in 2 models. The proportional hazards assumption was not violated. Table 1 shows the outcome of multivariable Cox regression of these models. Figure 1 is the visualization of the models in the form of nomograms. The predictors for each model and model parameters are listed below. Heterogeneity between centers was low, and a regular (fixed-effects) Cox regression was performed. The nomograms were converted to a web-based tool, accessible at www. EpilepsyPredictionTools.info/ttswithdrawal. Calibration of the models is visualized in Figures $1 C$ and D.

Factors predicting seizure recurrence were the following: (higher) age at withdrawal, (shorter) time to AED reduction (start tapering of the first AED), multifocal MRI abnormalities, interictal epileptiform discharges on EEG, and incomplete resection of the anatomical lesion (Table 1 , Figure 1); these factors combined into a prediction model had an adjusted concordancestatistic of 0.68 (95\% Cl 0.67-0.69). Internal-external cross-validation shows variation of the adjusted c-statistic between 0.61 and 0.69 , with wider confidence intervals (Table 2).

Factors predicting seizure freedom at final follow-up were the following: seizure frequency before surgery, (lower) number of AEDs used at time of surgery, and complete resection of the anatomical lesion (Table 1, Figure 1); these factors combined into a prediction model had an adjusted concordance-statistic of 0.73 (95\% Cl 0.72-0.75). Internal-external cross-validation shows variation of the adjusted c-statistic between 0.71 and 0.79 and wider confidence intervals (Table 2). 
Table 1 | Predicting the outcome of AED withdrawal in seizure-free children after epilepsy surgery.

\begin{tabular}{|c|c|c|c|c|}
\hline & \multicolumn{2}{|c|}{ Seizure recurrence } & \multicolumn{2}{|c|}{$\begin{array}{l}\text { Seizure-freedom at last } \\
\text { follow-up (seizure-free } \geq \\
1 \text { year) }\end{array}$} \\
\hline & HR $(95 \% \mathrm{Cl})$ & p-value & HR $(95 \% \mathrm{Cl})$ & p-value \\
\hline Time to reduction (months from surgery) & * & * & & \\
\hline \multicolumn{5}{|l|}{ MRI } \\
\hline focal abnormality or normal & Reference & & & \\
\hline multifocal abnormalities & $2.39(1.36-4.22)$ & 0.003 & & \\
\hline Age at withdrawal (years) & $1.06(1.01-1.10)$ & 0.008 & & \\
\hline \multicolumn{5}{|l|}{ Postoperative EEG } \\
\hline normal or not performed & Reference & & & \\
\hline interictal epileptiform discharges & $1.72(1.10-2.68)$ & 0.02 & & \\
\hline \multicolumn{5}{|l|}{ Complete resection of the anatomical lesion } \\
\hline complete or not determined & Reference & & Reference & \\
\hline incomplete & $2.46(1.55-3.39)$ & $<0.001$ & $0.23(0.11-0.51)$ & $<0.001$ \\
\hline $\begin{array}{l}\text { Seizure frequency (number of seizures per } \\
\text { day) }\end{array}$ & & & * & * \\
\hline Number of AEDs at time of surgery & & & $0.68(0.46-0.99)$ & 0.05 \\
\hline \multicolumn{5}{|l|}{ Model performance } \\
\hline $\begin{array}{l}\text { Adjusted c-statistic (95\% confidence } \\
\text { interval) }\end{array}$ & $0.68(0.67-0.69)$ & & $0.73(0.72-0.75)$ & \\
\hline Percentage optimism adjusted for & $2.8 \%$ & & $4.9 \%$ & \\
\hline Random effects measure of heterogeneity & 1.39 & & 1.25 & \\
\hline
\end{tabular}

AED, antiepileptic drug; c-statistic, concordance-statistic; $\mathrm{Cl}$, confidence interval; $\mathrm{EEG}$, electroencephalography; $\mathrm{HR}$, hazard ratio; $\mathrm{MRI}$, magnetic resonance imaging.

Multivariable models with strongest predictors for seizure recurrence and seizure freedom at last followup. Categorical variables with 3 or 4 items of which only 1 was significantly different from the reference category were dichotomized, so that the nonsignificant categories were collapsed with the reference category.

The random effects measure of heterogeneity can be interpreted as the hazard rate between 2 equally sized prognostic groups from 2 different cohorts. The values indicate relatively low heterogeneity.

* Not linearly modeled, restricted cubic splines used with 3 knots. For the effect size, see Figure 1. 
Table 2 | Internal-external cross-validation.

\begin{tabular}{|c|c|c|c|c|c|}
\hline & \multirow[b]{2}{*}{ City } & \multicolumn{2}{|c|}{$\begin{array}{l}\text { Internal validation (all } \\
\text { data minus indicated city) }\end{array}$} & \multicolumn{2}{|c|}{$\begin{array}{l}\text { External validation } \\
\text { (indicated city) }\end{array}$} \\
\hline & & $\begin{array}{l}\text { Adjusted } \\
\text { c-statistic }\end{array}$ & $95 \% \mathrm{Cl}$ & $\begin{array}{l}\text { Adjusted } \\
\text { c-statistic }\end{array}$ & $95 \% \mathrm{Cl}$ \\
\hline \multirow{4}{*}{$\begin{array}{l}\text { predicting seizure } \\
\text { recurrence }\end{array}$} & Bielefeld $(n=114)$ & 0.67 & $0.58-0.76$ & 0.69 & $0.60-0.78$ \\
\hline & Bonn $(n=124)$ & 0.61 & $0.56-0.66$ & 0.61 & $0.56-0.66$ \\
\hline & Milan $(n=131)$ & 0.69 & $0.61-0.77$ & 0.68 & $0.64-0.72$ \\
\hline & Utrecht $(n=87)$ & 0.69 & $0.63-0.75$ & 0.67 & $0.60-0.74$ \\
\hline \multirow{4}{*}{$\begin{array}{l}\text { predicting long-term } \\
\text { seizure-freedom }\end{array}$} & Bielefeld $(n=114)$ & 0.79 & $0.67-0.91$ & 0.75 & $0.62-0.88$ \\
\hline & Bonn $(n=120)$ & 0.71 & $0.61-0.81$ & 0.72 & $0.64-0.80$ \\
\hline & Milan $(n=131)$ & 0.73 & $0.66-0.80$ & 0.75 & $0.56-0.94$ \\
\hline & Utrecht $(\mathrm{n}=87)$ & 0.73 & $0.59-0.87$ & 0.73 & $0.63-0.83$ \\
\hline
\end{tabular}

The 4 largest cohorts within the data were chosen for internal-external cross-validation. Data from 1 of the indicated cities are temporarily removed, after which the model is refitted (training phase). Based on the remaining data, an internal validation is performed. External validation is performed on the removed data from one city. Subsequently, the removed data are placed back and the procedure is repeated for the next city, and the next, until 4 external validations have been performed.

Adjusted c-statistic: concordance-statistic, adjusted for overfitting using 200 bootstrap samples.

\section{Discussion}

We developed a prognostic tool to predict the seizure outcome after AED withdrawal in children who became seizure-free through epilepsy surgery, based on preselected factors that were identified through systematic review of the literature. The nomograms and the web-based tool, developed on a dataset from 766 children, are clinically useful when considering AED withdrawal in an individual patient. The predicted outcomes can serve as a basis to counsel children and caregivers when balancing the risks and benefits of AED withdrawal. The selected predictors differ between the 2 models. Of interest, 4 predictors of seizure recurrence were not predictive of eventual seizure outcome: the timing of AED withdrawal-as revealed in the original TimeToStop study report (Boshuisen et al. 2012)—the findings of preoperative MRI, the age at withdrawal, and postoperative EEG abnormalities. Two factors were related only to eventual seizure outcome: the preoperative seizure frequency had a U-shaped relation with seizure outcome, with the lowest and highest seizure frequencies related to lower chances of eventual seizure freedom, and the number of AEDs before surgery, which showed a linear relation with the outcome. The only factor predictive of both outcomes was the completeness of resection: incomplete resection of the anatomical lesion increased the hazard of seizure recurrence by a factor of 2.5 and decreased the hazard of eventual seizure freedom by a factor of 4 . 


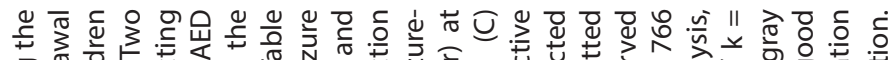

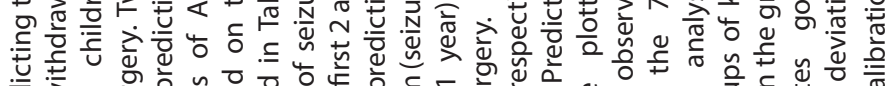

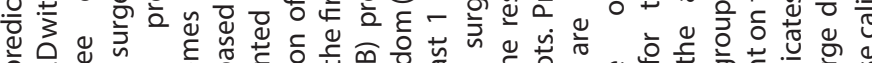

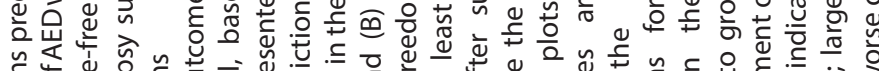

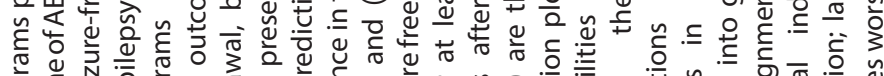

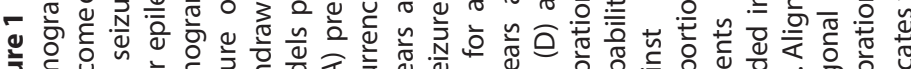

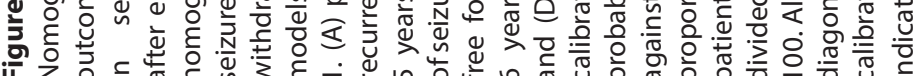
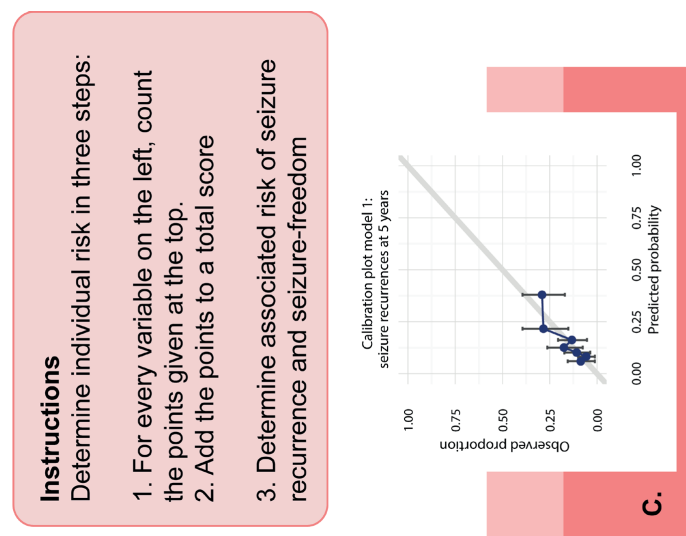

j

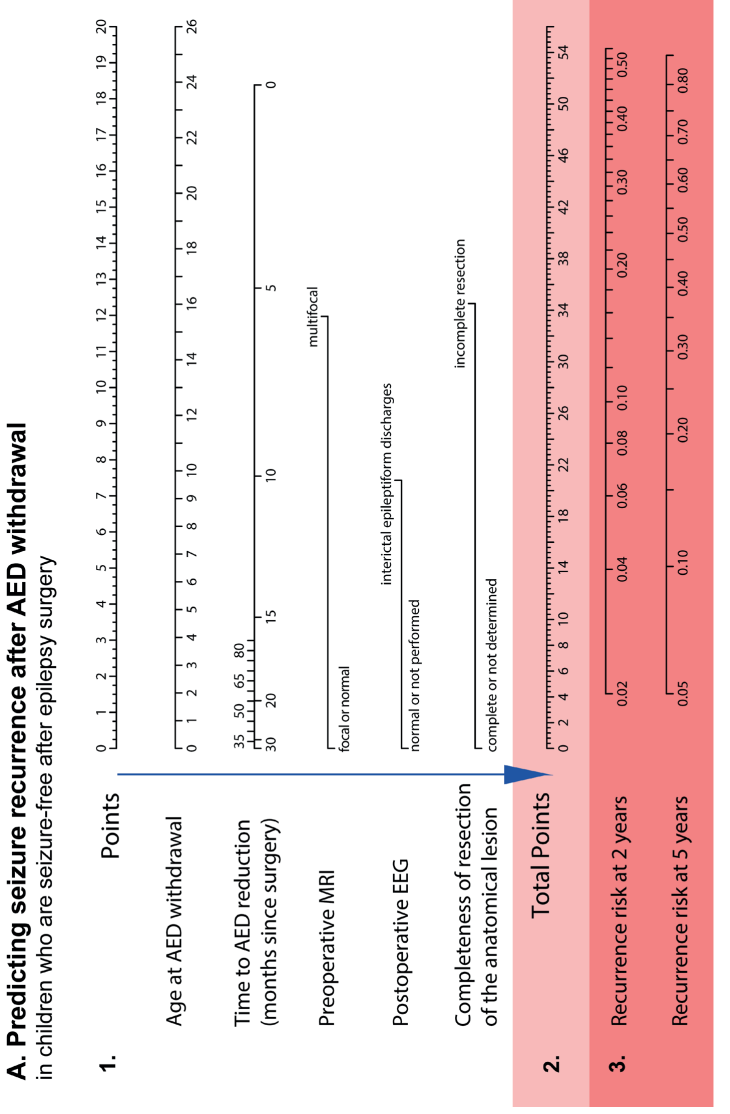

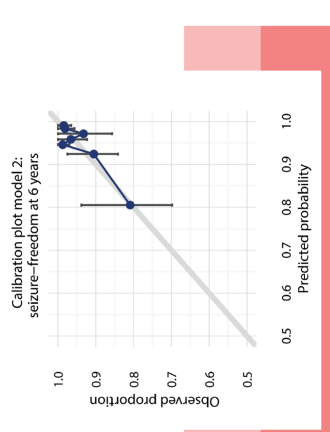

$\dot{0}$

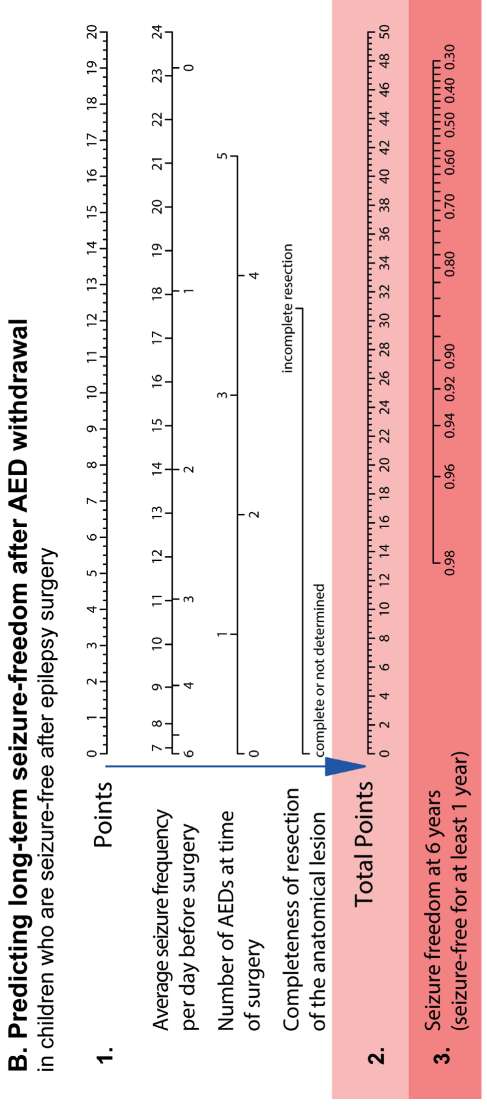


Model performance in terms of discrimination was best for predicting eventual seizure freedom, with an adjusted c-statistic of 0.73 . The calibration plot for this model shows that it can accurately identify a small proportion with increased risk, where most patients have a predicted chance between $95 \%$ and $100 \%$ of being seizure-free for at least 1 year at an average follow-up duration of 6 years after start of AED withdrawal. For predicting seizure recurrence, the adjusted c-statistic of 0.68 shows reasonable discriminatory ability of the model.

The current analysis has several limitations. First, although the analysis was performed on a Europe-wide multicenter cohort and bootstrap-samples were used to correct for overfitting, model performance should be tested in an external validation cohort, because the data were collected retrospectively. Second, one of the preselected potential predictors, "postoperative seizures," was not tested because none of the patients with this factor experienced seizure relapse. Although no parameters could be estimated, none of these 13 patients relapsed and 6 of 11 patients (55\%) were successful at discontinuing AEDs, which is similar to the $48 \%$ in the complete cohort. Therefore, the current data do not indicate an increased risk for patients with postoperative seizures, once it has been decided to withdraw AEDs. However, the time to reduction was almost twice as long for these patients, indicating that they had a substantial longer period of seizure freedom after the initial postsurgical relapse before reduction of AEDs. Finally, it is important to realize that postoperative seizure relapse risk has never been compared between patients who do, and those who do not, withdraw medication, in a prospective randomized manner. Our data, and all cohort studies reporting on postwithdrawal relapse rates, included only a selection of patients for whom the treating physician deemed it safe to start AED withdrawal. It cannot be excluded that recurrence rates, predicted by the current model, are somewhat higher when applied in a total cohort of unselected children who underwent epilepsy surgery. Therefore, the risk predictor is most reliably applied in patients in whom physicians already consider withdrawal of medication but want to balance its risks and benefits to better counsel the individual patient and his or her caregivers.

In conclusion, 2 prognostic models with moderate to good discriminative performance were created and converted to a clinically useful tool to predict the individual risk of seizure recurrence, and eventual seizure freedom, for children after epilepsy surgery. 


\section{Supplementary Table 1 | List of potential predictors}

\begin{tabular}{lll}
\hline & Potential predictor & References \\
\hline 1 & Age at withdrawal & 1 \\
2 & Time from surgery to reduction & $1-3$ \\
3 & Duration of epilepsy before surgery & 3,4 \\
4 & Absence of MRI abnormalities & 3 \\
5 & Multifocal MRI lesions & 2 \\
6 & Postoperative EEG interictal epileptiform discharges before AED withdrawal & $2,4,5,6$ \\
7 & Seizure frequency before surgery & 6 \\
8 & Presence of postoperative seizures (excluding'running down' seizures) & 3,7 \\
9 & Type of surgery (hemispherectomy) & 2 \\
10 & Etiology & 5 \\
11 & Incomplete resection & 2 \\
12 & Previous surgery & 2 \\
\hline
\end{tabular}

Potential predictors as identified in a systematic review of the literature. Adapted from: Lamberink $\mathrm{HJ}$, Otte WM, Geleijns K, Braun KPJ. Antiepileptic drug withdrawal in medically and surgically treated patients: a meta-analysis of seizure recurrence and systematic review of its predictors. Epileptic Disorders 2015; 17(3):211-228 with permission from John Libbey Eurotext.

AED, antiepileptic drug; EEG, electroencephalography; MRI, magnetic resonance imaging.

References:

1. Lee SY, Lee JY, Kim DW, Lee SK, Chung CK. Factors related to successful antiepileptic drug withdrawal after anterior temporal lobectomy for medial temporal lobe epilepsy. Seizure 2008; 17(1): 11-8.

2. Boshuisen K, Arzimanoglou A, Cross JH, et al. Timing of antiepileptic drug withdrawal and longterm seizure outcome after paediatric epilepsy surgery (TimeToStop): a retrospective observational study. Lancet Neurol 2012; 11(9): 784-91.

3. Park KI, Lee SK, Chu K, et al. Withdrawal of antiepileptic drugs after neocortical epilepsy surgery. Ann Neurol 2010; 67(2): 230-8.

4. Menon R, Rathore C, Sarma SP, Radhakrishnan K. Feasibility of antiepileptic drug withdrawal following extratemporal resective epilepsy surgery. Neurology 2012; 79(8): 770-6.

5. Rathore C, Panda S, Sarma PS, Radhakrishnan K. How safe is it to withdraw antiepileptic drugs following successful surgery for mesial temporal lobe epilepsy? Epilepsia 2011; 52(3): 627-35.

6. Yardi R, Irwin A, Kayyali H, et al. Reducing versus stopping antiepileptic medications after temporal lobe surgery. Ann Clin Transl Neurol 2014; 1(2): 115-23.

7. Berg AT, Vickrey BG, Langfitt JT, et al. Reduction of AEDs in postsurgical patients who attain remission. Epilepsia 2006; 47(1): 64-71. 


\section{Supplementary Table 2 | Missing values}

\begin{tabular}{ll}
\hline Variable & Number of missing values \\
\hline Sex & 0 \\
Seizure recurrence & 0 \\
Time to seizure recurrence & 0 \\
MRI & 0 \\
Etiology & 0 \\
Completeness of resection & 0 \\
Postoperative seizures & 0 \\
Age at surgery & 1 \\
Follow-up duration after start AED withdrawal & 6 \\
Seizures in last year of follow-up & 6 \\
Type of surgery & 6 \\
Time to AED reduction & 6 \\
Number of AEDs before surgery & 6 \\
Age at start AED withdrawal & 7 \\
Age at onset of epilepsy & 8 \\
Seizure frequency & 11 \\
EEG after surgery & 15 \\
Duration of epilepsy until surgery & 17 \\
Previous epilepsy surgery & 50 \\
\hline
\end{tabular}

Number of missing values in the original data. Missing values were imputed with multiple imputations using chained equations.

$A E D$, antiepileptic drug; EEG, electroencephalography; $M R I$, magnetic resonance imaging. 


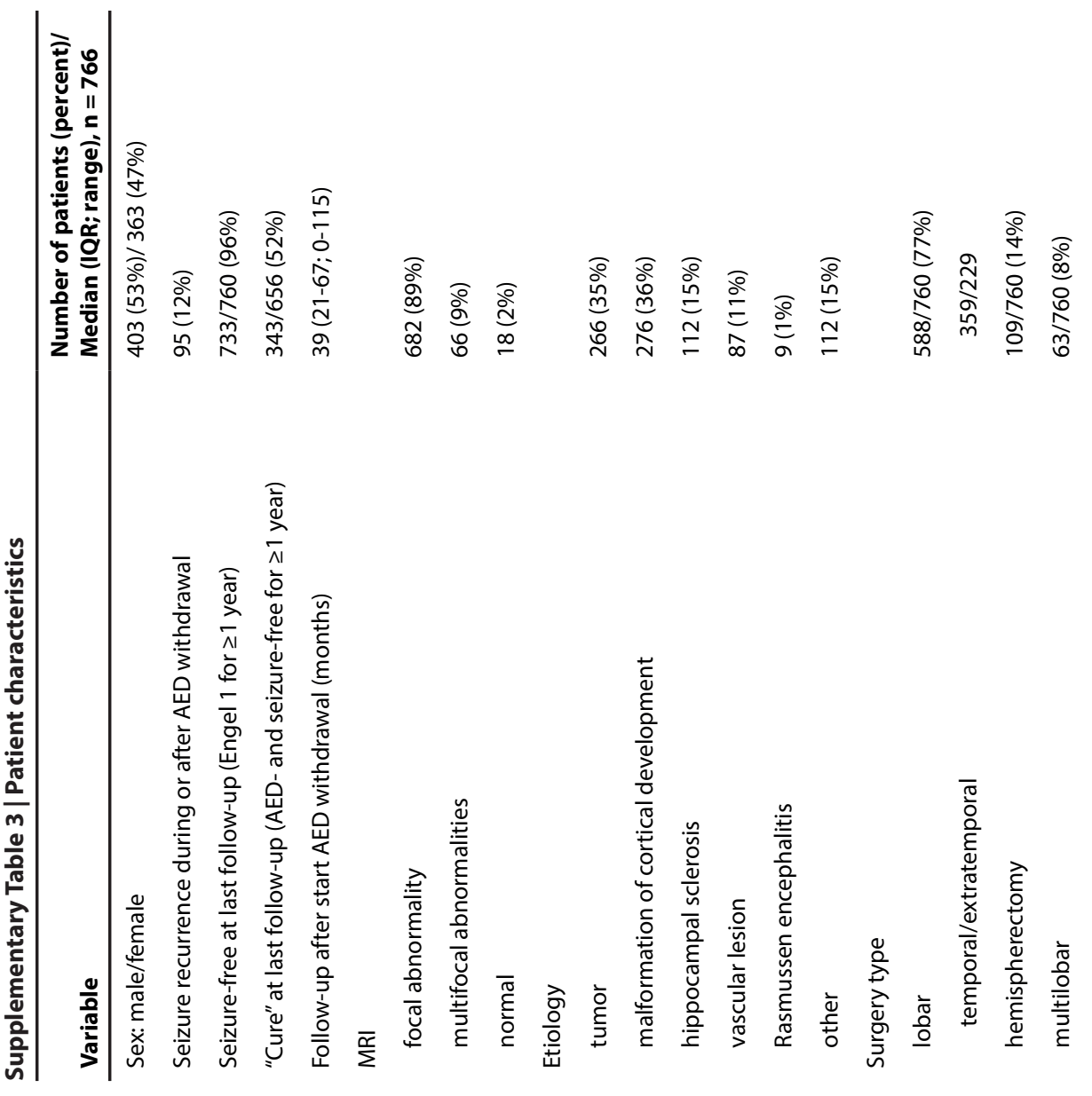




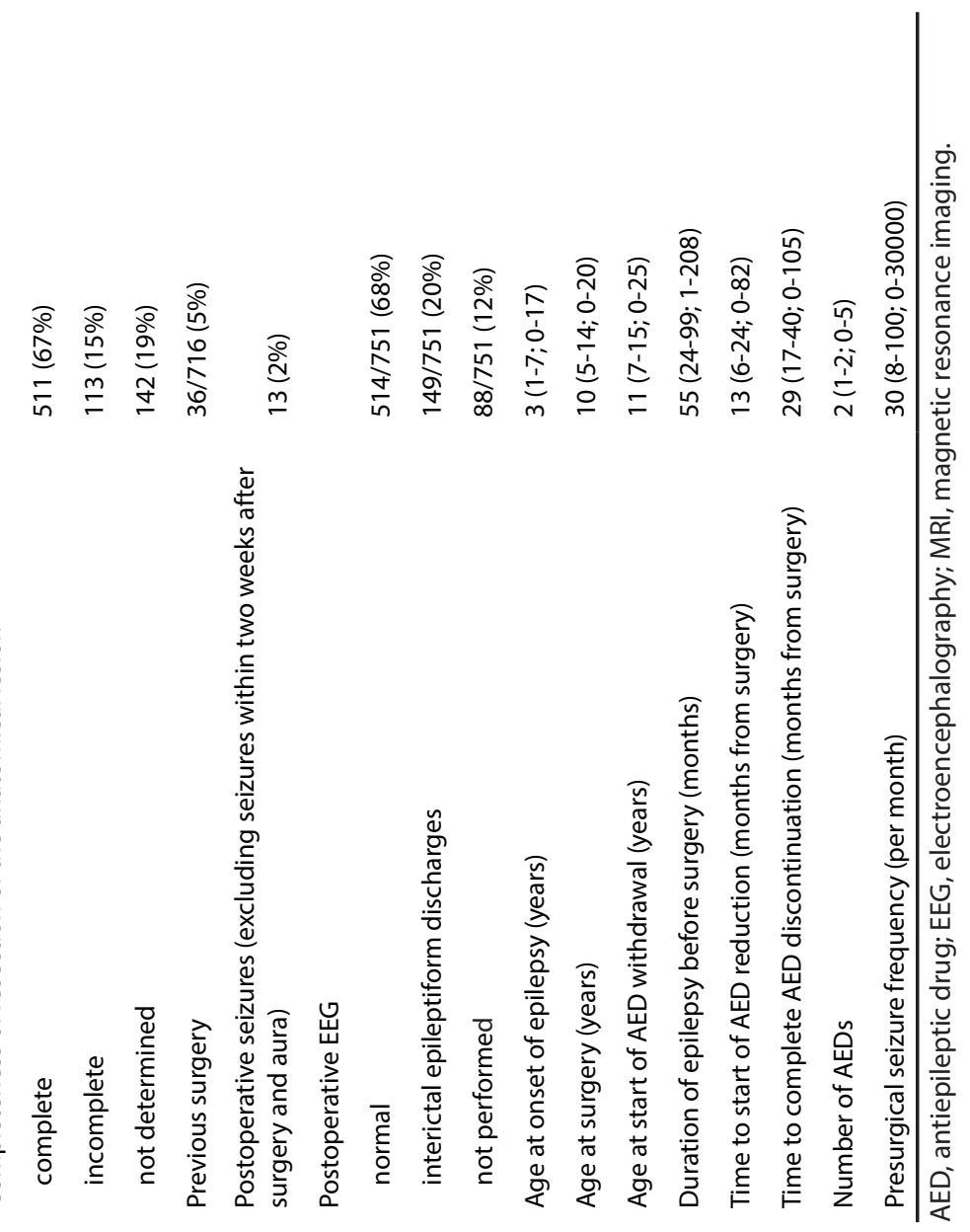




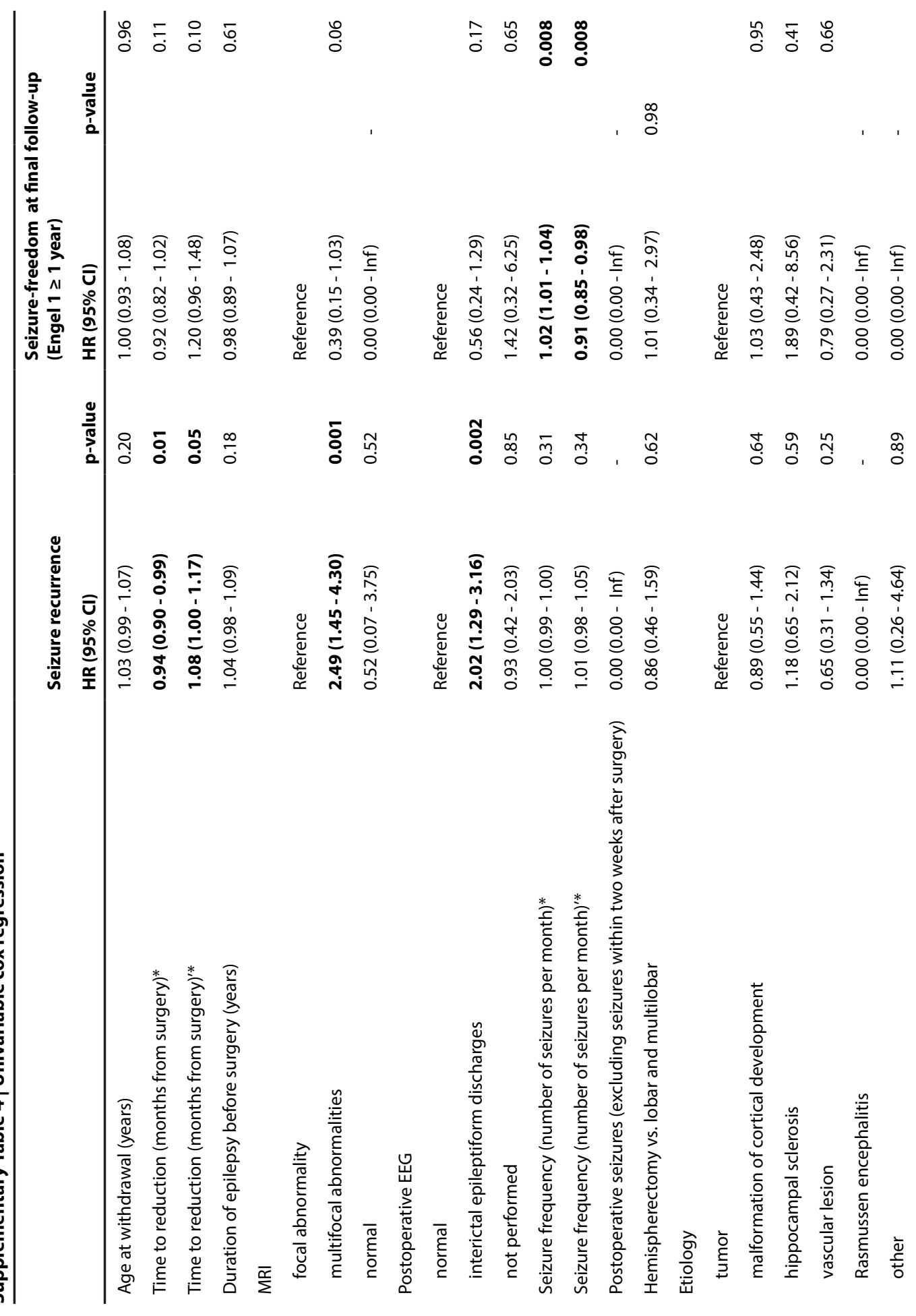









\section{TimeToStop Study Group}

Austria. M Feucht, G Gröppel (Medical University Vienna, Vienna). France. P Kahane, L Minotti (University Hospital Grenoble, Grenoble); A Arzimanoglou, P Ryvlin, E Panagiotakaki, J de Bellescize, K Ostrowsky-Coste (University Hospitals of Lyon, Lyon); E Hirsch, M Valenti (University Hospital Strasbourg, Strasbourg). Germany. T Polster (Bethel Epilepsy Center, Bielefeld); R Sassen, C Hoppe, S Kuczaty, C Elger (University Hospital, Bonn, Bonn); S Schubert (University Hospital Heidelberg, Heidelberg); K Strobl, T Bast (Epilepsy Center Kork, Kehl-Kork). Italy. C Barba, R Guerrini, F Giordano (Meyer Children's Hospital, University Hospital, Florence); S Francione, D Caputo (Claudio Munari Epilepsy Surgery Center Ospedale Niguarda, Milan). The Netherlands. K Boshuisen, K P J Braun, C S P M Uiterwaal, O van Nieuwenhuizen, F S Leijten, P C van Rijen (University Medical Center Utrecht, Utrecht). Switzerland. M Seeck (Neurology Clinic, University Hospital Geneva, Geneva). Turkey. D Yalnizoglu, G Turanli, M Topcu (Hacetteppe University Faculty of Medicine, Department of Pediatric Neurology, Ankara); C Özkara, M Uzan (Istanbul University, Cerrahpasa Medical Faculty, Istanbul). United Kingdom. J H Cross, L D’Argenzio, W Harkness (University College London Institute of Child Health, Great Ormond Street Hospital for Children NHS Foundation Trust, London). 

GENERAL DISCUSSION 

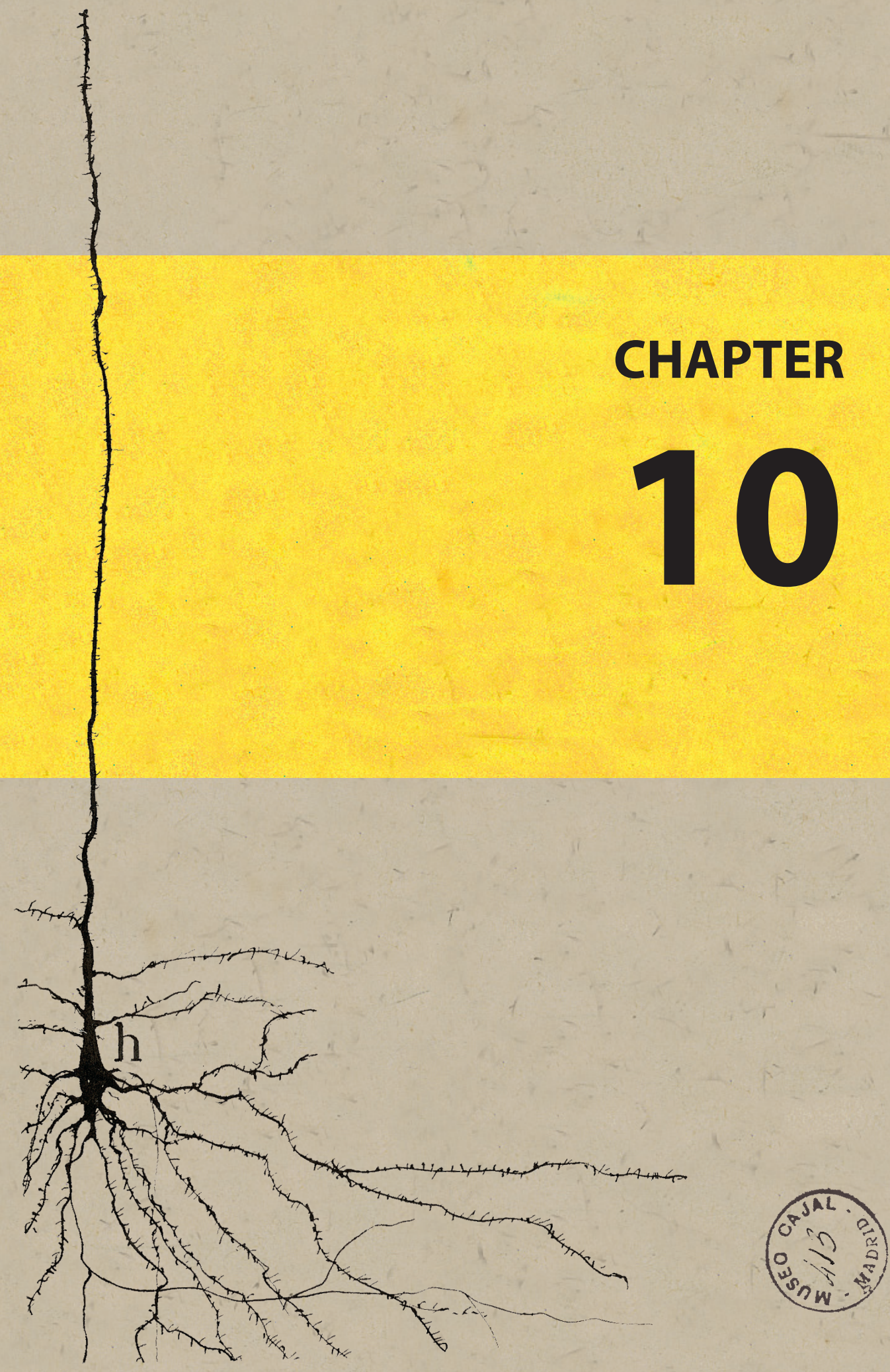


\section{Discussion}




\section{DISCUSSION}

The general aim of this thesis is to increase knowledge on the risks and benefits of, and policies on, AED withdrawal. Specific objectives were to develop tools to predict individual risks of AED withdrawal, explore postoperative medication policies, investigate long-term cognitive effects of the use of these drugs in children, and to study potential cognitive benefits of postoperative AED withdrawal. This general discussion will start by putting our findings in context and exploring the implications of this thesis. Furthermore, some additional practical and methodological considerations will be presented. The remaining part will focus on some of the remaining questions and important topics for future studies will be highlighted.

\section{Main findings in context}

BOX 1 summarizes the main findings regarding AED withdrawal.

\section{PART I. AED withdrawal in patients without surgery}

From the literature review in Chapter 2 it became evident that on average 34\% of medically treated people who attempt AED withdrawal will have a seizure recurrence in the next four years after drug reduction. Although average risks are useful for giving an indication of the risk in the entire population, a large drawback of using these pooled relapse rates to counsel patients on their individual risk, is that it is based on many different types of populations. The risks reported in these populations ranged from $10 \%$ to $66 \%$ of patients experiencing seizure recurrence after withdrawal. Therefore, an individualized approach to risk estimation is preferred.

For those seizure free on AED treatment alone, Chapter 4 offered a tool which answers the following question: if a person with epilepsy stops AED treatment, what is the risk that seizures return within the following two and five years, and what is the chance that this person is still (or again) seizure free ten years after medication cessation? In Chapter 4 we report on nomograms which were created using an individual participant data (IPD) meta-analysis, to answer these questions for the individual patient. An easy way to compute these risks is to go to the website www.epilepsypredictiontools.info/aedwithdrawal, which is a direct translation of the prediction tools in Chapter 4. The impact of this prediction model is illustrated by the commentaries that were written in response to its publication (French 2017; Brigo 2017), the extent to which it is discussed in a recent review on the topic (Schachter 2018), and the over 12,000 visits to the prediction tool website between May 2017 and May 2019 (not counting visits to the other prediction tools on the website). 
Chapter 3 adds another item to the list of reasons to consider AED withdrawal in children: it revealed a potential link between cumulative effects of AED treatment in children on their eventual IQ. We found a negative association between AED treatment and intelligence. This association remained after correction for many potential confounding variables. The more drugs, and the longer they are taken, the stronger the effect. The average patient in that study had around five drug-years, which would mean an average IQ decrease of six points according to the results. However, since this is the first study to show such long-term effects and the study design has its limitations, it is very important that the results are replicated in a prospective study. Potential side-effects should not be discussed out of context without regarding the benefits, because it is well known that epilepsy itself, and continuing seizures, are also related to cognitive problems (Braun 2017), and treatment is often necessary also to reduce the risk of complications of seizures itself and even the risk on mortality (Mohanraj et al. 2006). This context is paramount when interpreting the results of the study.

The results from Chapter 4 highlight some other important aspects of AED withdrawal, for example regarding the timing of tapering. Previous studies, in particular randomized trials, compared early withdrawal, usually defined as after a remission period of less than two years, with late withdrawal. These studies are summarized in a recent Cochrane meta-analysis (Strozzi et al. 2015) and the conclusion is that the risk of recurrence is 1.3 times higher after early withdrawal. This is proven for children with partial seizures only, because there are no randomized trials in adults, or in children with generalized seizures. What our study shows is that this dichotomy between early and late withdrawal does not portray the true extent of the relation between timing and risk. Instead, the message is: every additional year of seizure freedom reduces the risk of recurrence. What is regarded as an unacceptable risk after two years of seizure freedom by an individual might be seen as acceptable after five years of seizure freedom. Another important finding regards previous unsuccessful withdrawal attempts: even when a previous attempt failed - i.e. seizures recurred and AEDs were restarted - this will not affect the chances of successful AED withdrawal in the future. 


\section{BOX 1 | Clinically relevant main findings}

Meta-analysis shows that the average cumulative seizure relapse risk is approximately $34 \%$ percent for patients who became seizure free after AED treatment, and $24 \%$ in surgical cohorts (Chapter 2).

Cumulative drug-load of AEDs in children with epilepsy is negatively associated with their eventual intellectual functioning (Chapter 3 ).

By combining data from 10 cohorts and 1769 patients in an IPD meta-analysis, we have developed a reliable individualized prediction model and online calculation tool to help counseling patients who consider withdrawing AEDs after having reached seizure-freedom (Chapter 4).

Although numbers and complexity of paediatric epilepsy surgery cases have increased over the last two decades in our single-center nation-wide cohort, seizure-freedom remained high, with $74 \%$ at two and $65 \%$ at five years, and the proportion of children who successfully withdrew AEDs doubled over time (Chapter 5).

From an unprecedentedly large European epilepsy surgery cohort we have learned that seizure-outcomes largely differ between aetiologies, epilepsy duration negatively affects outcome, and children significantly more often become seizure- and medication-free than adults (Chapter 6).

Postoperative medication withdrawal policies in children have changed drastically over the last years, leading to premature discontinuation of a prospective randomized control trial to compare cognitive outcomes of children who withdrew AEDs early versus late after surgery (Chapter 7); patients and doctors consider late withdrawal not justified (Chapter 8).

We developed an individualized prediction tool to calculate seizure relapse risks after postoperative AED withdrawal in children (Chapter 9). 


\section{PART II. AED withdrawal after epilepsy surgery}

The studies described in this section taught us several important aspects about the policies that are employed regarding postoperative AED withdrawal, and ended with a prediction tool for postoperative AED withdrawal in children. Epilepsy surgery renders $66 \%$ of patients seizure free (Chapter 6), and for these patients it should be decided whether and when to start AED withdrawal. Most chapters contain retrospective studies in which we observe which medication policies were used in the past. In Chapter 5 for example, an important shift was demonstrated in medication policy over time. Where before the year 2000 only $14 \%$ of children were AED free two years after surgery, this number more than doubled for the years 2001-2011. Despite an increase in complexity of paediatric surgical cases and more rapid AED withdrawal, seizure outcomes remained excellent. The proportion of AED-free children is expected to have increased further in recent years, because in 2012 the retrospective TimeToStop study was published, which showed for the first time that in children early withdrawal is not correlated with long-term seizure outcome (Boshuisen et al. 2012). As a logical sequel to that study, we initiated a prospective randomized trial to compare the cognitive outcome of children after early (4 months after surgery) versus late (12 months after surgery) withdrawal, and to unambiguously confirm safety of early withdrawal (Chapter 7). The preliminary termination of this trial reflects an even stronger recent shift in AED policies and patient and parent perception after the results of the TimeToStop retrospective study became known: none of the eligible patients were included, because almost all parents demanded early withdrawal and refused to be randomized into a treatment arm that required continuing AEDs for an entire year (Chapter 8). The survey in Chapter 8 furthermore highlights that also most paediatric epileptologists did not think waiting for 12 months after successful epilepsy surgery before AED withdrawal is justified. The average answer distilled from the responses in the survey would say: if early withdrawal is safe, then we should not wait for one, or even two years before the patient is liberated from AEDs. Combining this information leads to the conclusion that current practice in paediatric epilepsy surgery is to start early with AED withdrawal, provided there are no important predictors of surgical failure.

Another observation regarding medication policy is the large difference between adults and children. Chapter 6 compared seizure and medication outcomes in large multicenter European cohorts of operated children and adults in this regard, and children were more likely to be medication free five years after surgery. Children had a higher relative chance of seizure freedom compared to adults, and their chance of reaching AED freedom was even higher. The same pattern was also shown in an international survey among neurologists: paediatric neurologists more often recommended drug tapering and would taper early in children with a low expected recurrence risk. In addition, physicians with over ten years experience more often recommended AED withdrawal (Bartolini, Majidi, and Koubeissi 2018). 
In Chapter 6, data from 37 centres across Europe not only revealed that epilepsy aetiology largely determines seizure outcomes over time and that epilepsy duration is an important independent predictor of seizure outcome, it also demonstrated that there is no consensus regarding the timing of postoperative AED withdrawal, with a wide range of policies between centres. Although the proportions of seizure free patients per centre were symmetrically distributed around the average of $60-70 \%$, the number of seizure- and drug-free patients at five years varied from $0 \%$ to $70 \%$ for the participating centres. This indicates that in some centres AEDs were tapered in almost all seizure free patients, whereas in others this was attempted in none. The international survey in Chapter 8 confirms that there are worldwide geographical differences in policy. In contrast to European paediatric epilepsy specialists, nonEuropean physicians start later with AED withdrawal for children on monotherapy as well as on polytherapy. It thus seems that the timing of AED withdrawal is as much dependent on country, centre or physician as on clinical factors related to the epilepsy.

As discussed in Part l, average risks are only to a limited extent informative for the individual patient, and the same counts for the average of $24 \%$ recurrences after epilepsy surgery (Chapter 2). With Chapter 9 we have provided a practical tool to define this risk in more detail for the individual patient, in the setting of paediatric epilepsy surgery. This model still requires external validation. A recent review on postoperative management of AEDs stated the following: "several important pieces of information are still missing to rationally guide the post-surgical management of the antiepileptic treatment: in fact, criteria to identify the best candidates in whom AEDs can be discontinued or to predict a high risk of relapse after tapering AEDs are lacking" (Rubboli et al. 2017). Our prediction tool is another step toward rationally counselling patients and their parents on the decision when or whether to withdraw medication.

\section{Counselling the individual patient}

\section{Understanding risks}

When using a prediction tool to counsel a patient, the physician should be aware of several aspects. First, understanding risks can be quite difficult, especially when calculated for a binary event; in this case, seizures will recur or not. A hypothetical risk of $15 \%$ is easily interpreted as 'it will probably not happen'. A great danger in this intuitive approach is that the consequences of an eventual seizure recurrence are not discussed thoroughly. There are several aspects that, in my opinion, should be laid out in every discussion regarding potential AED withdrawal with patient. These are mentioned in BOX 2. 
BOX 2 | Questions to ask your patient when discussing AED withdrawal.

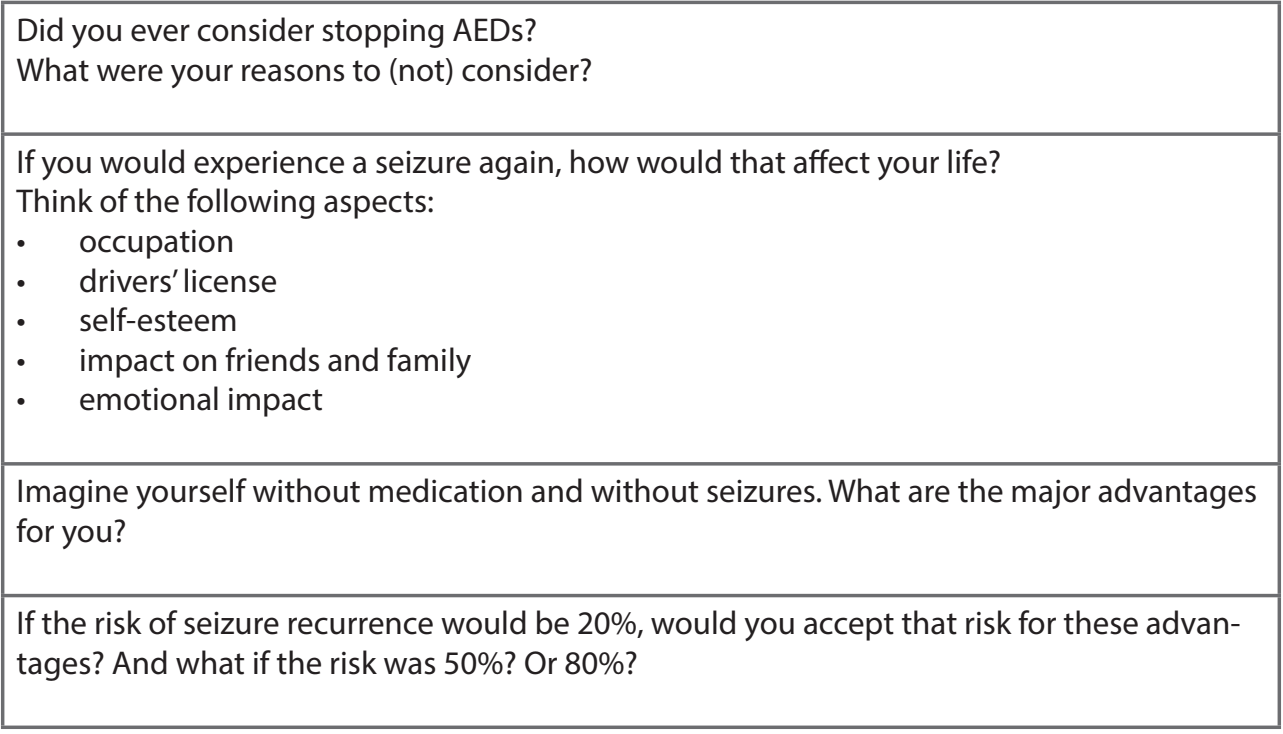

No matter how low the risk of seizure recurrence, the consequences of seizure recurrence should be discussed. Vice versa, no matter how high the risk of recurrence is, it should be discussed what the patient would gain by stopping AEDs. Failing to address either may lead to regret afterward - because of an unexpected seizure recurrence in the case of low predicted risk, or because they feel they do not need the medication anymore but fail to discuss this with their doctor. The latter is beautifully illustrated in a series of interviews, in which Ross (aged 10 years) tells that he stopped his medication because he thought the drugs had cured him:

"I[nterviewer]: Did you think you were going to have seizures again when you stopped taking it or did you think they'd gone away?

Ross: I hadn't had one for about a year and a half so I thought 'OK, that's close enough'. Although Ross thought his medication had cured his epilepsy, he went on to have another seizure and admitted to his mum that he had been putting his medicine down the sink." (Webster 2017)

Framing and choice

Almost forty years ago, Tversky and Kahneman (the latter is author of the enlightening book 'Thinking, Fast and Slow') have described the so-called framing effect, which is the effect of presenting risks positively (e.g. $85 \%$ chance) or negatively (e.g. 15\% risk) on the choice made afterward (Tversky and Kahneman 1981). Because deciding on AED withdrawal is ultimately the patient's choice, such a framing effect would have an impact on their decision. Gong and 
colleagues have performed a review of medical literature, to investigate whether such an effect also exists in medical decision-making, but unfortunately were unable to find a definite answer (Gong et al. 2013). Another study addressed the framing effect in the specific context of AED withdrawal: Jacoby and colleagues asked 62 patients about their preference on AED withdrawal, before and after they spoke with their physician about their individualized seizure recurrence risk (Jacoby et al. 1993) as computed with a prediction tool presented by the MRC AED withdrawal study group (Medical Research Council 1993). The counselling was framed either negatively or positively, and patients were classified as wishing to withdraw, to continue or to be uncertain, before and after counselling (Table 1). Because of the small difference between the (small) groups, a conclusion regarding framing could not be drawn (left part of Table 1). What is more striking in these results is that most patients who were beforehand inclined to start AED withdrawal, and those who were not certain yet, decided on a more conservative approach to continue AED treatment after being presented a personalized actual risk percentage, as calculated by a risk calculation tool (right part of Table 1).

Table 1 | AED withdrawal risk counselling and framing - results from (Jacoby et al. 1993)

\begin{tabular}{|c|c|c|c|c|c|}
\hline \multirow[b]{2}{*}{$\begin{array}{l}\text { Preference } \\
\text { beforehand }\end{array}$} & \multirow[b]{2}{*}{$\begin{array}{l}\text { Framing } \\
\text { strategy }\end{array}$} & \multicolumn{2}{|c|}{ Preference afterward } & \multicolumn{2}{|l|}{ Regardless of framing } \\
\hline & & Withdraw & Continue & $\begin{array}{l}\text { Preference for AED } \\
\text { continuation before } \\
\text { counselling }\end{array}$ & $\begin{array}{l}\text { Preference for AED } \\
\text { continuation after } \\
\text { counselling }\end{array}$ \\
\hline \multirow[t]{2}{*}{ Withdraw } & Positive & 1 & 3 & $0 / 62$ & $11 / 62(18 \%)$ \\
\hline & Negative & 4 & 8 & & \\
\hline \multirow[t]{2}{*}{ Continue } & Positive $^{1}$ & 1 & 8 & $18 / 62(29 \%)$ & $17 / 62(27 \%)$ \\
\hline & Negative $^{1}$ & 0 & 9 & & \\
\hline \multirow[t]{3}{*}{ Uncertain } & Positive & 5 & 12 & $0 / 62$ & $22 / 62(35 \%)$ \\
\hline & Negative & 1 & 10 & & \\
\hline & TOTAL & 12 & 50 & $18 / 62(29 \%)$ & $50 / 62(81 \%)$ \\
\hline
\end{tabular}

'These two rows may have been switched because of incomplete information: "Before counselling, 18 patients wanted to continue AEDs, of whom equal numbers received positively and negatively framed risk information. After counselling, all but one of them still wanted to continue." (Jacoby et al. 1993)

\section{Adjusting to the new normal life}

Adjusting to the "new normal" life without medication after AED withdrawal (Chiu et al. 2014) can be a challenge in itself. Restrictions in life-style can have a large impact on the person with epilepsy and their close surrounding. Although stopping treatment only temporarily increases the risk of relapse, the effect of epilepsy may linger for a longer period of time. For parents of children with epilepsy for example, stress levels are high, but they go down in the years after successful AED withdrawal (Operto et al. 2019). The parental stress levels did not normalize, however - they stayed elevated (Operto et al. 2019). This indicates that even when most of the 
risk of seizures is reduced, their child's condition has not disappeared from the parents minds. For adolescents with epilepsy AED withdrawal does give them a sense of liberation (Chiu et al. 2014). In a series of interviews with 11 children, subjects experienced a freedom from the constraints connected to antiepileptic therapy (Chiu et al. 2014). The interviewed adolescents described now being able to join the activities of their friends without any restrictions and feeling free (Chiu et al. 2014). Of course, these results only apply to children who have become seizure free, as the study did not interview adolescents who had experienced a relapse due to AED withdrawal.

\section{Methodological considerations in the presentation of prediction tools}

A recent article in the British Medical Journal described the advantages and disadvantages of different ways of presenting a prediction tool (Bonnett et al. 2019). A nomogram, for example, does not require a computer or phone. Furthermore, in contrast to simplifying a model into a simple risk score, it retains continuous variables without categorization, and allows for risk estimation at multiple time points. A potential difficulty is that proper understanding of a nomogram and performing the risk calculation may take some time. Lastly, addition errors may be made since the calculation is performed manually. Bonnett and colleagues (Bonnett et al. 2019) state that web-based tools such as those on www.epilepsypredictiontools.info can be an easy way to compute multiple risks at the same time, but also stress that easy access may also mean that such a tool could be used by people for whom it is not intended, potentially leading to misinterpretation. Although we developed the prediction tools for use by the neurologist, people with epilepsy could also use the tool from home. Before making the tool available online we have discussed this possibility in our research team and it was decided to keep the website open without restrictions, to maximize its reach and applicability. The website indicates that the tool is developed for medical professionals only, and it does not advocate any treatment decisions; it only provides an estimation of the risk. By presenting both nomograms and translating the model into a web-tool, we have made use of the advantages of both methods.

\section{Directions for future research}

The content of this thesis has given some answers to clinical questions, expanded the knowledge on the context of AED withdrawal, and provided new tools for the clinician to better inform patients. Nevertheless, many questions remain, and new questions may actually have arisen to offer guidance for future research. Also, some of the studies had limitations that might be overcome by different study designs. In this section I offer my personal view on important steps to take in future research.

Two chapters in this thesis were dedicated mainly to cognitive side effects of AEDs, and much 
remains unclear. Most of the research on the cognitive side-effect profile of AEDs was done ten to forty years ago, when the profile of prescribed drugs was very different from now (Chen et al. 2018). Newer classes of AEDs seem to have less side-effects, but most evidence is circumstantial or of low quality. Another complicating factor in this type of research is that there are many potential confounding effects of epilepsy itself on cognitive performance. Prospective and longitudinal studies, that measure potential side effects before and during administration of AEDs, are needed to get a better insight in the effect of the medication for an individual. Comparably, the side effects could be measured before and after stopping AEDs. Cumulative long-term effects of AEDs are extremely difficult to discern from cumulative longterm effects of epilepsy, or the condition from which epilepsy arose. The best evidence would come from placebo-controlled randomized and blinded trials, which for an indication such as epilepsy would not be ethical since continued seizures are associated with considerable risks. AEDs, however, are prescribed for other conditions beside epilepsy, for example in many psychiatric disorders they are thought to have beneficial effects (Ovsiew 2004). However, these disorders also come with (temporary) cognitive problems, making them less suitable for the proposed study. Migraine is another condition for which AEDs are indicated as second-line treatment (Bagnato and Good 2016). A randomized clinical trial using AEDs for migraine may be useful to give information on the cognitive side effects.

Where the studies in this thesis looked at AED withdrawal in the context of seizure freedom and potentially 'resolved' epilepsy, there is another field of research that is interested in AED withdrawal. Within the context of epilepsy surgery, AED withdrawal can be used to provoke seizures, and therefore serve as a diagnostic tool to gather information on the seizure onset zone. This is usually done during admission to an epilepsy monitoring unit, in which there is a continuous recording of combined EEG and video images, so clinical features can be correlated to neurophysiological patterns (Rheims and Ryvlin 2014). The goal of this procedure is to capture seizures and interictal EEG patterns that can guide epilepsy surgery. However, concerns have been raised about the safety of AED withdrawal in the monitoring unit, regarding the risk of seizure clusters, status epilepticus, and injuries due to falling, for example. (Di Gennaro et al. 2012; Dobesberger et al. 2011; Ryvlin et al. 2013; Rubboli et al. 2015), and few centers have a protocol in place on how to withdraw AEDs for this purpose (Kobulashvili et al. 2016). Practices vary, to an extent that some centers start tapering medication in the week(s) before admission to the monitoring unit, and some only start during the video-EEG registration. To study the effect of the timing and speed of AED withdrawal in this setting in relation to the occurrence of complications and successful monitoring, we launched the NeedToStop study in 2017 (Netherlands Trial Registry number NL6445, https://www.trialregister.nl/trial/6445), a European observational prospective study.

There is much to gain from future prognostic studies regarding outcomes of AED withdrawal. 
Many studies in the past tried to identify predictors of outcome based on a small sample of patients, but these small studies are of limited value when assessing risk factors individually (see also Chapter 2). The multitude of studies did allow an IPD meta-analysis, but the simple approach of assessing the predictive value of a combination of basic clinical variables in small single-center cohorts has been repeated many times, and more of these studies will probably not gain new knowledge. Still, at least eight studies have been published on the topic since 2017, when our IPD-meta-analysis became available (Choi et al. 2019; Syvertsen et al. 2019; Healy et al. 2018; Kerkhof et al. 2019; Rathore et al. 2018; Tang et al. 2017; Zhang et al. 2018; Ou et al. 2018). Some of these publications provide new and potentially useful data, for instance because the population studied is essentially different from the ones studied before, including people with glioma (Kerkhof et al. 2019) and with Juvenile Myoclonic Epilepsy (Syvertsen et al. 2019; Healy et al. 2018). However, some of the research seems to be driven by the availability of data, instead of the need for answers. The right question to ask today is not ' what are predictors of seizure recurrence after AED withdrawal?', rather we should be searching for factors that could improve prognostic accuracy.

Prediction of AED withdrawal outcomes can be improved on multiple levels. First, the prediction tool from Chapter 4 is based mainly on data from the 1990's. Definitions of epilepsy and aetiology were different, and great technological advances have been made since. This provides ample room for improvement of the prediction model, especially because the c-statistic of 0.64 indicates a moderate performance. When updating the existing prediction model, special attention should be given to the added value of modern imaging techniques, to the current definition of aetiologies, and the advances that have been made in the field of epilepsy genetics. When assessing the predictive value of imaging, previous studies (as discussed in Chapters 2 and 4) mainly looked at a dichotomous value of the presence or absence of MRI abnormalities. This is a great simplification of the potential of MRI diagnoses, which might underutilize its predictive capacities. As for aetiology, an early meta-analysis already found that the diagnosis of 'remote symptomatic etiology' was related to a higher risk of seizure recurrence (Berg and Shinnar 1994). However, this classification is outdated, and future studies should classify it according to the 2017 ILAE definition into structural, genetic, infectious, metabolic, immune, and unknown aetiology (Scheffer et al. 2017). This is where $\mathrm{MRI}$, genetic and metabolic laboratory tests can be utilized in sync to define the aetiology as a possible predictor in future research. Two other factors of interest would be the type of AED and the dosage before withdrawal. Lastly, the advances made in genetics should be taken into account in future predictive studies, as already attempted for the prediction of AED treatment response (Silva-Alves et al. 2017). Potentially, predictive factor finding studies could focus on identifying specific gene alleles that are associated with the outcome of AED withdrawal, although it may be difficult to grasp a complex factor as genetics and fruitfully implement this into a prediction model. 
The complexity of many questions, in combination with the scarcity of data, makes international collaboration essential for real progress in the future. Pooling multiple small-scale datasets into one larger IPD meta-analysis such as in Chapter 4 is a first step. International collaboration on gathering retrospective data in a uniform manner at a large scale such as in Chapter 6 is another. The most difficult but crucial step will be to define the core research questions in an international context, and gather data for this prospectively. Even though gathering prospective data is costly and time-consuming, only well-designed studies will ultimately improve clinical practice.

In conclusion, AED withdrawal - whether in the context of seizure freedom on medication only or after epilepsy surgery - is a topic on which opinions and practices differ, and an approach tailored to the individual patient is needed. The scientific community can improve prognostic validity of prediction tools, identify the best timing of postoperative AED withdrawal, and work toward drugs with less side effects. The final decision regarding stopping or continuing AED treatment however will always be for the person with epilepsy: are the gains of stopping AED treatment worth the risk of a seizure recurrence? That is a question science can never answer in their stead. 



\section{ADDENDUM}

References

Summary

Nederlandse samenvatting

List of publications

About the author

Dankwoord 


\section{REFERENCES}

Afshari, Daryoush, and Nasrin Moradian. 2012."Evaluating the Rate of Recurrence of Epilepsy after Therapy Discontinuation in 2-Year Seizure-Free." International Journal of Neuroscience 122: 598601. https://doi.org/10.3109/00207454.2012.694936.

Ahmed, Ikhlaaq, Thomas Pa Debray, Karel Gm Moons, and Richard D Riley. 2014. “Developing and Validating Risk Prediction Models in an Individual Participant Data Meta-Analysis." BMC Medical Research Methodology 14 (3). https://doi.org/10.1186/1471-2288-14-3.

Al-Kaylani, Muhammad, Peter Konrad, Barry Lazenby, Bennett Blumenkopf, and Bassel Abou-Khalil. 2007. "Seizure Freedom off Antiepileptic Drugs after Temporal Lobe Epilepsy Surgery." Seizure 16: 95-98. https://doi.org/10.1016/j.seizure.2006.10.007.

Aldenkamp, Albert, Frank Besag, Giuseppe Gobbi, Rochelle Caplan, David W Dunn, and Matti Sillanpää. 2016. "Psychiatric and Behavioural Disorders in Children with Epilepsy ( ILAE Task Force Report): Adverse Cognitive and Behavioural Effects of Antiepileptic Drugs in Children." Epileptic Disord 18: s55-67.

Aldenkamp, AP, WCJ Alphers, G Blennow, D Elmqvist, J Heijbel, HL Nilsson, P Sandstedt, B Tonnby, L Wahlander, and E Wosse. 1993. "Withdrawal of Antiepileptic Medication in Children - Effects on Cognitive Function: The Multicenter Holmfrid Study." Neurology 43: 41-50.

Aldenkamp, AP, WC Alpherts, P Sandstedt, G Blennow, D Elmqvist, J Heijbel, HL Nilsson, B Tonnby, L Wahlander, and E Wosse. 1998. "Antiepileptic Drug-Related Cognitive Complaints in Seizure-Free Children with Epilepsy before and after Drug Discontinuation." Epilepsia 39 (10): 1070-74.

Alsemari, Abdulaziz, Faisal Al-otaibi, Salah Baz, Ibrahim Althubaiti, Hisham Aldhalaan, David Macdonald, Tareq Abalkhail, et al. 2014. “Epilepsy Surgery Series: A Study of 502 Consecutive Patients from a Developing Country." Epilepsy Research and Treatment 2014: 286801. https://doi. org/10.1155/2014/286801.

Altunbasak, Sakir, Ömer Artar, Refik Burgut, and Dincer Yildiztas. 1999. “Relapse Risk Analysis after Drug Withdrawal in Epileptic Children with Uncomplicated Seizures." Seizure 8: 384-89.

Alvarez, Norberto. 1989. "Discontinuance of Antiepileptic Medicatinos in Patients with Developmental Disability and Diagnosis of Epilepsy." American Journal on Mental Retardation 93 (6): 593-99.

Åndell, Eva, Torbjörn Tomson, Sofia Carlsson, Eva Hellebro, Tomas Andersson, Cecilia Adelöw, and Per Åmark. 2015. “The Incidence of Unprovoked Seizures and Occurrence of Neurodevelopmental Comorbidities in Children at the Time of Their First Epileptic Seizure and during the Subsequent Six Months." Epilepsy Research 113: 140-50. https://doi.org/10.1016/j.eplepsyres.2015.04.002.

Arts, WFM, LH Visser, MCB Loonen, AT Tjiam, H Stroink, PM Stuurman, and DCJ Poortvliet. 1988. “Followup of 146 Children with Epilepsy after Withdrawal of Antiepileptic Therapy." Epilepsia 29 (3): 244-50.

Bagnato, Francesca, and Janine Good. 2016. "The Use of Antiepileptics in Migraine Prophylaxis.” Headache Currents 56 (3): 603-15.

Baker, G.A., Ann Jacoby, D Buck, C Stalgis, and D Monnet. 1997. "Quality of Life of People with Epilepsy: A European Study." Epilepsia 38 (3): 353-62. https://doi.org/10.1111/j.1528-1157.1997.tb01128.x.

Baron, Reuben M, and David A Kenny. 1986. "The Moderator-Mediator Variable Distinction in Social Psychological Research: Conceptual , Strategic , and Statistical Considerations." Journal of Personality and Social Psychology 51 (6): 1173-82.

Bartolini, Luca, Shahram Majidi, and Mohamad Z Koubeissi. 2018. “Uncertainties from a Worldwide Survey on Antiepileptic Drug Withdrawal after Seizure Remission." Neurology 8 (2): 108-15. https:// doi.org/10.1212/CPJ.0000000000000441.

Bath, Kevin G, and Helen E Scharfman. 2013. "Impact of Early Life Exposure to Antiepileptic Drugs on Neurobehavioral Outcomes Based on Laboratory Animal and Clinical Research." Epilepsy \& Behavior 26 (3): 427-39. https://doi.org/10.1016/j.yebeh.2012.10.031.

Baud, Maxime O, Thomas Perneger, R Attila, M C Pensel, Christian E Elger, B Rydenhag, Kristina Malmgren, et al. 2018. "European Trends in Epilepsy Surgery." Neurology 91 (2): e96-106. https:// doi.org/10.1212/WNL.0000000000005776. 
Beghi, Ettore. 2011. "AED Discontinuation May Not Be Dangerous in Seizure-Free Patients." Journal of Neural Transmission 118 (2): 187-91. https://doi.org/10.1007/s00702-010-0528-y.

Beghi, Ettore, Giorgia Giussani, Salvatore Grosso, Alfonso ludice, Angela La Neve, Francesco Pisani, Luigi M Specchio, et al. 2013. "Withdrawal of Antiepileptic Drugs: Guidelines of the Italian League Against Epilepsy." Epilepsia 54 (SUPPL.7): 2-12. https://doi.org/10.1111/epi.12305.

Beghi, Ettore, Giorgia Giussani, and Josemir W Sander. 2015. "The Natural History and Prognosis of Epilepsy." Epileptic Disorders 17 (3): 243-53.

Beghi, Ettore, and Dieter Schmidt. 2013. "When and How to Stop Antiepileptic Drugs." Epileptology 1 (1): 17-20. https://doi.org/10.1016/j.epilep.2012.07.001.

Bell, Gail S, Jane De Tisi, Juan Carlos Gonzalez-Fraile, Janet L Peacock, Andrew W McEvoy, William F J Harkness, Jacqueline Foong, et al. 2017. “Factors Affecting Seizure Outcome after Epilepsy Surgery: An Observational Series." Journal of Neurology, Neurosurgery \& Psychiatry 88: 933-40. https://doi.org/10.1136/jnnp-2017-316211.

Berg, Anne T, Samuel F Berkovic, Martin J Brodie, Jeffrey Buchhalter, J Helen Cross, Walter Van Emde Boas, J Jr Engel, et al. 2010. "Revised Terminology and Concepts for Organization of Seizures and Epilepsies: Report of the ILAE Commission on Classification and Terminology, 2005-2009." Epilepsia 51 (4): 676-85. https://doi.org/10.1111/j.1528-1167.2010.02522.x.

Berg, Anne T, John T Langfitt, Susan S Spencer, and Barbara G Vickrey. 2007. "Stopping Antiepileptic Drugs after Epilepsy Surgery: A Survey of U.S. Epilepsy Center Neurologists." Epilepsy \& Behavior 10: 219-22. https://doi.org/10.1016/j.yebeh.2006.12.001.

Berg, Anne T, Gary W Mathern, Richard A Bronen, Robert K Fulbright, Francis Dimario, Francine M Testa, and Susan R Levy. 2009. "Frequency, Prognosis and Surgical Treatment of Structural Abnormalities Seen with Magnetic Resonance Imaging in Childhood Epilepsy." Brain 132: 2785-97. https://doi. org/10.1093/brain/awp187.

Berg, Anne T, and Shlomo Shinnar. 1994. "Relapse Following Discontinuation of Antiepileptic Drugs: A Meta-Analysis.pdf." Neurology 44: 601-8.

Berg, Anne T, Barbara G. Vickrey, John T. Langfitt, Michael R. Sperling, Shlomo Shinnar, Carl Bazil, Thaddeus Walczak, and Susan S. Spencer. 2006. "Reduction of AEDs in Postsurgical Patients Who Attain Remission." Epilepsia 47 (1): 64-71. https://doi.org/10.1111/j.1528-1167.2006.00371.x.

Berg, Anne T, Frank A Zelko, Susan R Levy, and Francine M Testa. 2012. "Age at Onset of Epilepsy, Pharmacoresistance, and Cognitive Outcomes: A Prospective Cohort Study." Neurology 79: 138491. https://doi.org/10.1212/WNL.0b013e31826c1b55.

Bien, Christian G, Anna L Raabe, Johannes Schramm, Albert Becker, Horst Urbach, and Christian E Elger. 2013. "Trends in Presurgical Evaluation and Surgical Treatment of Epilepsy at One Centre from 1988 - 2009." Journal of Neurology, Neurosurgery \& Psychiatry 84 (1): 54-61. https://doi. org/10.1136/jnnp-2011-301763.

Bittigau, Petra, Marco Sifringer, Kerstin Genz, Ellen Reith, Dana Pospischil, Suresh Govindarajalu, Mark Dzietko, et al. 2002. "Antiepileptic Drugs and Apoptotic Neurodegeneration in the Developing Brain." Proc. Natl. Acad. Sci. U.S.A. 99 (23): 15089-94.

Bjellvi, Johan, Roland Flink, Bertil Rydenhag, and Kristina Malmgren. 2015. “Complications of Epilepsy Surgery in Sweden 1996-2010: A Prospective, Population-Based Study." Journal of Neurosurgery JNS 122 (3): 519-25. https://doi.org/10.3171/2014.9.JNS132679.

Bjellvi, Johan, Ingrid Olsson, Kristina Malmgren, and Karin Wilbe Ramsay. 2019. “Epilepsy Duration and Seizure Outcome in Epilepsy Surgery - A Systematic Review and Meta-Analysis." Neurology Pub ahead. https://doi.org/10.1212/WNL.0000000000007753.

Blümcke, Ingmar, R Spreafico, G Haaker, R Coras, K Kobow, Christian G Bien, M Pfäfflin, et al. 2017. "Histopathological Findings in Brain Tissue Obtained during Epilepsy Surgery." The New England Journal of Medicine 377: 1648-56. https://doi.org/10.1056/NEJMoa1703784.

Bonnett, Laura J, A. Shukralla, C. Tudur-Smith, P. R. Williamson, and A. G. Marson. 2011. “Seizure Recurrence after Antiepileptic Drug Withdrawal and the Implications for Driving: Further Results from the MRC Antiepileptic Drug Withdrawal Study and a Systematic Review." Journal of Neurology, Neurosurgery \& Psychiatry 82 (12): 1328-33. https://doi.org/10.1136/jnnp.2010.222885.

Bonnett, Laura J, Kym I E Snell, Gary S Collins, and Richard D Riley. 2019. "Guide to Presenting Clinical Prediction Models for Use in Clinical Settings." BMJ 365: 1737. https://doi.org/10.1136/bmj.1737. 
Boshuisen, Kim, Alexis Arzimanoglou, J Helen Cross, Cuno S P M Uiterwaal, Tilman Polster, Onno Van Nieuwenhuizen, and Kees P J Braun. 2012. "Timing of Antiepileptic Drug Withdrawal and Long-Term Seizure Outcome after Paediatric Epilepsy Surgery (TimeToStop): A Retrospective Observational Study." The Lancet Neurology 11 (9): 784-91. https://doi.org/10.1016/S14744422(12)70165-5.

Boshuisen, Kim, Herm J Lamberink, M.M.J. van Schooneveld, J Helen Cross, Alexis Arzimanoglou, I. van der Tweel, Karin Geleijns, C.S.P.M. Uiterwaal, and Kees P J Braun. 2015. “Cognitive Consequences of Early versus Late Antiepileptic Drug Withdrawal after Pediatric Epilepsy Surgery, the TimeToStop (TTS) Trial: Study Protocol for a Randomized Controlled Trial." Trials 16 (1). https://doi.org/10.1186/ s13063-015-0989-2.

Boshuisen, Kim, MMJ van Schooneveld, FSS Leijten, GAP de Kort, PC van Rijen, PH Gosselaar, Onno Van Nieuwenhuizen, and Kees P J Braun. 2010. “Contralateral MRI Abnormalities Affect Seizure and Cognitive Outcome after Hemispherectomy." Neurology 75: 1623-30. https://doi.org/10.1212/ WNL.0b013e3181fb4400.

Boshuisen, Kim, Monique M J Van Schooneveld, Cuno S P M Uiterwaal, J Helen Cross, Sue Harrison, Tilman Polster, Marion Daehn, et al. 2015. "Intelligence Quotient Improves after Antiepileptic Drug Withdrawal Following Pediatric Epilepsy Surgery." Annals of Neurology 78 (1): 104-14. https://doi. org/10.1002/ana.24427.

Bouma, P A D, A C B Peters, and O F Brouwer. 2002. “Long Term Course of Childhood Epilepsy Following Relapse after Antiepileptic Drug Withdrawal." Journal of Neurology, Neurosurgery \& Psychiatry 72: 507-10. https://doi.org/10.1136/jnnp.72.4.507.

Braathen, Gunnar, and Hans Melander. 1997. “Early Discontinuation of Treatment in Children with Uncomplicated Epilepsy: A Prospective Study with a Model for Prediction of Outcome." Epilepsia 38 (5): 561-69.

Braun, Kees P J. 2017. "Preventing Cognitive Impairment in Children with Epilepsy." Curr Opin Neurol 30 (2): 140-47. https://doi.org/10.1097/WCO.0000000000000424.

Braun, Kees P J, and Dieter Schmidt. 2014. "Stopping Antiepileptic Drugs in Seizure-Free Patients." Curr Opin Neurol 27: 219-26. https://doi.org/10.1097/WCO.0000000000000075.

Brelie, Christian von der, Michael P Malter, Pitt Niehusmann, Christian E Elger, Marec von Lehe, and Johannes Schramm. 2013. "Surgical Management and Long-Term Seizure Outcome after Epilepsy Surgery for Different Types of Epilepsy Associated with Cerebral Cavernous Malformations." Epilepsia 54 (9): 1699-1706. https://doi.org/10.1111/epi.12327.

Brigo, Francesco. 2017. "Antiepileptic Drug Withdrawal Risk Calculator." Practical Neurology 17 (4): 332-33. https://doi.org/10.1136/practneurol-2017-001715.

Bromley, Rebecca L, and Gus A Baker. 2017. "Fetal Antiepileptic Drug Exposure and Cognitive Outcomes." Seizure 44: 225-31. https://doi.org/10.1016/j.seizure.2016.10.006.

Buna, Donna K. 1998. "Antiepileptic Drug Withdrawal - a Good Idea?" Pharmacotherapy 18 (2): 235-41.

Buuren, Stef Van, and Karin Groothuis-Oudshoorn. 2011. "Multivariate Imputation by Chained Equations." Journal Of Statistical Software 45 (3): 1-67. https://doi.org/10.1177/0962280206074463.

Callaghan, Noel, Andrew Garrett, and Timothy Goggin. 1988. "Withdrawal of Anticonvulsant Drugs in Patients Free of Seizures for Two Years: A Prospective Study." The New England Journal of Medicine 318 (15): 942-46.

Camfield, Peter, and Carol Camfield. 2005. “The Frequency of Intractable Seizures after Stopping AEDs in Seizure-Free Children with Epilepsy." Neurology 64: 973-75.

Cardoso, Tania M O, Fernando Cendes, and Carlos A M Guerreiro. 2003. "Is Low Antiepileptic Drug Dose Effective in Long-Term Seizure-Free Patients?" Arquivos de Neuro-Psiquiatria 61 (3 A): 566-73. https://doi.org/10.1590/S0004-282X2003000400008.

Carpay, JA, J Vermeulen, H Stroink, OF Brouwer, A C B Peters, Albert Aldenkamp, CA van Donselaar, and WFM Arts. 2002. “Parent-Reported Subjective Complaints in Children Using Antiepileptic Drugs: What Do They Mean?" Epilepsy \& Behavior 3 (4): 322-29.

Caviedes, Benigno E, and Jose L Herranz. 1998. "Seizure Recurrence and Risk Factors after Withdrawal of Chronic Antiepileptic Therapy in Children." Seizure 7: 107-14.

Chadwick, David, J Taylor, and T Johnson. 1996. "Outcomes after Seizure Recurrence in People with WellControlled Epilepsy and the Factors That Influence It. The MRC Antiepileptic Drug Withdrawal 
Group." Epilepsia 37 (11): 1043-50. https://doi.org/10.1111/j.1528-1157.1996.tb01023.x.

Chassoux, Francine, Eric Artiges, Franck Semah, Agathe Laurent, Elisabeth Landré, Baris Turak, Philippe Gervais, Badia-Ourkia Helal, and Bertrand Devaux. 2017."18F-FDG-PET Patterns of Surgical Success and Failure in Mesial Temporal Lobe Epilepsy." Neurology 88: 1045-53.

Chen, Yi-Hua, Hung-Yi Chiou, Herng-Ching Lin, and Hsiu-Li Lin. 2009. "Affect of Seizures during Gestation on Pregnancy Outcomes in Women with Epilepsy." Arch. Neurol. 66 (8): 979-84.

Chen, Zhibin, Martin J Brodie, Danny Liew, and Patrick Kwan. 2018. "Treatment Outcomes in Patients with Newly Diagnosed Epilepsy Treated with Established and New Antiepileptic Drugs - a 30-Year Longitudinal Cohort Study." JAMA Neurology 75 (3): 279-86. https://doi.org/10.1001/ jamaneurol.2017.3949.

Chern, Joshua J, Akash J Patel, Andrew Jea, Daniel J Curry, and Youssef G Comair. 2010. "Surgical Outcome for Focal Cortical Dysplasia: An Analysis of Recent Surgical Series - a Review." J Neurosurg 6: 452-58. https://doi.org/10.3171/2010.8.PEDS10145.

Chiu, Ya-ping, Tzu-ying Lee, Kuang-lin Lin, and Virginia L Laadt. 2014. “Adjusting to a Seizure-Free'new Normal' Life Following Discontinuation of Antiepileptic Drugs during Adolescence." Epilepsy \& Behavior 33: 54-58. https://doi.org/10.1016/j.yebeh.2014.02.009.

Choi, S A, S Y Kim, W J Kim, Y K Shim, H Kim, H Hwang, J E CHoi, et al. 2019. “Antiepileptic Drug Withdrawal after Surgery in Children with Focal Cortical Dysplasia: Seizure Recurrence and Its Predictors." Journal of Clinical Neurology 15 (1): 84-89.

Christensen, Jakob, TK Grønborg, MJ Sørensen, D Schendel, ET Parner, LH Pedersen, and M Vestergaard. 2013. “Prenatal Valproate Exposure and Risk of Autism Spectrum Disorders and Childhood Autism." JAMA 309 (16): 1696-1703.

Cloppenborg, Thomas, Theodor W May, Ingmar Blümcke, Susanne Fauser, Philip Grewe, Johanna L Hopf, Thilo Kalbhenn, et al. 2019. “Differences in Pediatric and Adult Epilepsy Surgery: A Comparison at One Center from 1990 to 2014." Epilepsia 60 (2): 233-45. https://doi.org/10.1111/epi.14627.

Cossu, M, G Lo Russo, S Francione, R Mai, L Nobili, I Sartori, L Tassi, et al. 2008. “Epilepsy Surgery in Children: Results and Predictors of Outcome on Seizures." Epilepsia 49 (1): 65-72.

Cross, J Helen. 2002. “Epilepsy Surgery in Childhood." Epilepsia 43: Suppl 3:65-70.

D’Andrea Meira, Isabella, Tayla Taynan Romão, Henrique Jannuzzelli Pires do Prado, Lia Theophilo Krüger, Maria Elisa Paiva Pires, and Priscila Oliveira da Conceicao. 2019. “Ketogenic Diet and Epilepsy: What We Know so Far." Frontiers in Neuroscience 13. https://doi.org/10.3389/fnins.2019.00005.

Das, Nirupam, Meenakshi Dhanawat, and Sushant K Shrivastava. 2012. "An Overview on Antiepileptic Drugs." Drug Discoveries and Therapeutics 6 (4): 178-93. https://doi.org/10.5582/ddt.2012.v6.4.178.

Delgado, MR, AR Riela, J Mills, A Pitt, and R Browne. 1996. “Discontinuation of Antiepileptic Drug Treatment after Two Seizure-Free Years in Children with Cerebral Palsy." Pediatrics 97: 192-97.

Deoni, Sean C L, Douglas C Dean, Justin Remer, Holly Dirks, and Jonathan O'Muircheartaigh. 2015. "Cortical Maturation and Myelination in Healthy Toddlers and Young Children." Neurolmage 115: 147-61. https://doi.org/10.1016/j.neuroimage.2015.04.058.

Desikan, Rahul S, Florent Se, Bruce Fischl, Brian T Quinn, Bradford C Dickerson, Deborah Blacker, Randy L Buckner, et al. 2006. “An Automated Labeling System for Subdividing the Human Cerebral Cortex on MRI Scans into Gyral Based Regions of Interest." Neurolm 31: 968-80. https://doi.org/10.1016/j. neuroimage.2006.01.021.

Dobesberger, Judith, Gerald Walser, Iris Unterberger, Klaus Seppi, Giorgi Kuchukhidze, Julia Larch, Gerhard Bauer, et al. 2011. "Video-EEG Monitoring: Safety and Adverse Events in 507 Consecutive Patients." Epilepsia 52 (3): 443-52. https://doi.org/10.1111/j.1528-1167.2010.02782.x.

Dooley, J, K Gordon, P Camfield, C Camfield, and E Smith. 1996. “Discontinuation of Anticonvulsant Therapy in Children Free of Seizures for 1 Year: A Prospective Study." Neurology 46: 969-74.

Dwivedi, Rekha, Bhargavi Ramanujam, P Sarat Chandra, Savita Sapra, Sheffali Gulati, Mani Kalaivani, Ajay Garg, et al. 2017. "Surgery for Drug-Resistant Epilepsy in Children." The New England Journal of Medicine 377: 1639-47. https://doi.org/10.1056/NEJMoa1615335.

Eadie, M J. 2012. "Sir Charles Locock and Potassium Bromide." J R Coll Physicians Edinb 42: 274-79. https:// doi.org/10.4997/JRCPE.2012.317.

Edelvik, Anna, Bertil Rydenhag, Ingrid Olsson, Roland Flink, Eva Kumlien, and Kristina Malmgren. 2013. "Long-Term Outcomes of Epilepsy Surgery in Sweden - A National Prospective and Longitudinal 
Study." Neurology 81: 1244-51.

Edey, Stephan, Nicholas Moran, and Lina Nashef. 2014. "SUDEP and Epilepsy-related Mortality in Pregnancy." Epilepsia 55 (7): e72-74. https://doi.org/10.1111/epi.12621.

Ehrhardt, P, and WI Forsythe. 1989. "Prognosis after Grand Mal Seizures: A Study of 187 Children with Three-Year Remissions." Developmental Medicine and Child Neurology 31: 633-39.

Elsharkawy, AE, F Behne, F Oppel, H Pannek, R Schulz, M Hoppe, G Pahs, et al. 2008. “Long-Term Outcome of Extratemporal Epilepsy Surgery among 154 Adult Patients." J Neurosurg 108: 676-86. https:// doi.org/10.3171/JNS/2008/108/4/0676.

Emerit, MB, M Riad, and M Hamon. 1992. "Trophic Effects of Neurotransmitters during Brain Maturation." Biol Neonate 62 (4): 193-201.

Emerson, Ronald, Bernard J D'Souza, Eileen P Vining, Kenton R Holden, E David Mellits, and John M Freeman. 1981. "Stopping Medication in Children with Epilepsy: Predictors of Outcome." The New England Journal of Medicine 304 (19): 1125-29.

Engel, J Jr, GD Cascino, PCV Ness, TB Rasmussen, and LM Ojemann. 1993. “Outcome with Respect to Epileptic Seizures." In Surgical Treatment of the Epilepsies, edited by J Jr Engel. NY, USA: Raven Press.

Engel, J Jr, and International League Against Epilepsy. 2001. "A Proposed Diagnostic Scheme for People with Epileptic Seizures and with Epilepsy: Report of the ILAE Task Force on Classification and Terminology." Epilepsia 42 (6): 796-803.

Engel, J Jr, P Van Ness, T B Rasmussen, and L M Ojemann. 1993. “Outcome with Respect to Epileptic Seizures." In Surgical Treatment of the Epilepsies, edited by J Jr Engel, 2nded., 609-621. Edition Raven Press; New York.

Englot, Dario J, and Edward F Chang. 2014. "Rates and Predictors of Seizure Freedom in Resective Epilepsy Surgery: An Update." Neurosurg Rev 37: 389-405. https://doi.org/10.1007/s10143-0140527-9.

Englot, Dario J, S J Han, M T Lawton, and Edward F Chang. 2011. "Predictors of Seizure Freedom in the Surgical Treatment of Supratentorial Cavernous Malformations." J Neurosurg 115: 1169-74. https:// doi.org/10.3171/2011.7.JNS11536.

Englot, Dario J, D Ouyang, PA Garcia, NM Barbaro, and EF Chang. 2012. “Epilepsy Surgery Trends in the United States, 1990 - 2008." Neurology 78: 1200-1206.

Erba, Giuseppe, Lorenzo Moja, Ettore Beghi, Paolo Messina, and Elisabetta Pupillo. 2012.“Barriers toward Epilepsy Surgery. A Survey among Practicing Neurologists." Epilepsia 53 (1): 35-43. https://doi. org/10.1111/j.1528-1167.2011.03282.x.

Escayg, Andrew, and Alan L Goldin. 2010. "Sodium Channel SCN1A and Epilepsy: Mutations and Mechanisms." Epilepsia 51 (9): 1650-58. https://doi.org/10.1111/j.1528-1167.2010.02640.x.

European Medicines Agency. 2018. New Measures to Avoid Valproate Exposure in Pregnancy Endorsed: Member State Representatives Agree New Restrictions and Pregnancy Prevention Programme.

Farwell, JR, Y Jack Lee, DG Hirtz, SI Sulzbacher, JH Ellenberg, and KB Nelson. 1990. “Phenobarbital for Febrile Seizures - Effects on Intelligence and on Seizure Recurrence." The New England Journal of Medicine 322 (364-369).

Fischl, Bruce, and Anders M Dale. 2000. “Measuring the Thickness of the Human Cerebral Cortex from Magnetic Resonance Images." Proc. Natl. Acad. Sci. U.S.A. 97 (20): 11050-55.

Fischl, Bruce, David H Salat, Evelina Busa, Marilyn Albert, Megan Dieterich, Christian Haselgrove, Andre Van Der Kouwe, et al. 2002. "Whole Brain Segmentation: Neurotechnique Automated Labeling of Neuroanatomical Structures in the Human Brain." Neuron 33: 341-55.

Fisher, Robert S, Carlos Acevedo, Alexis Arzimanoglou, Alicia Bogacz, J Helen Cross, Christian E Elger, Jerome Engel Jr, et al. 2014. "ILAE Official Report: A Practical Clinical Definition of Epilepsy." Epilepsia 55 (4): 475-82. https://doi.org/10.1111/epi.12550.

Fisher, Robert S, J Helen Cross, Jacqueline A French, Norimichi Higurashi, Edouard Hirsch, FE Jansen, Lieven Lagae, et al. 2017. "Operational Classification of Seizure Types by the ILAE- Position Paper of the ILAE Commission for Classification and Terminology." Epilepsia 58 (4): 522-30.

Flon, P De, E Kumlien, C Reuterwall, and P Mattsson. 2010. "Empirical Evidence of Underutilization of Referrals for Epilepsy Surgery Evaluation." Eur. J. Neurol. 17: 619-25. https://doi.org/10.1111/j.14681331.2009.02891.x.

Freitag, Hedwig, and Ingrid Tuxhorn. 2005. “Cognitive Function in Preschool Children after Epilepsy 
Surgery: Rationale for Early Intervention." Epilepsia 46 (4): 561-67.

French, Jacqueline A. 2017. "Withdrawal of Antiepileptic Drugs: An Individualised Approach." The Lancet Neurology 16 (7): 493-94. https://doi.org/10.1016/S1474-4422(17)30136-9.

Gafoor, V Abdul, Kondanath Saifudheen, and James Jose. 2014. “Recurrence Rate of Seizure Following Discontinuation of Anti-Epileptic Drugs in Patients with Normal Long Term Electroencephalography." Annals of Indian Academy of Neurology 17 (3): 277-80.

Galimberti, C A, R Manni, L Parietti, E Marchioni, and A Tartara. 1993. “Drug Withdrawal in Patients with Epilepsy: Prognostic Value of the EEG." Seizure 2 (3): 213-20.

Gebremariam, Ayele, Worku Mengesha, and Fikre Enqusilassie. 1999. "Discontinuing Anti-Epileptic Medication(s) in Epileptic Children: 18 versus 24 Months." Annals of Tropical Paediatrics 19: 93-99.

Geerts, Ada T, J. M F Niermeijer, A. C B Peters, W. F M Arts, O. F. Brouwer, H. Stroink, E. A. J. Peeters, and C. A. Van Donselaar. 2005. "Four-Year Outcome after Early Withdrawal of Antiepileptic Drugs in Childhood Epilepsy." Neurology 64 (12): 2136-38. https://doi.org/10.1212/01. WNL.0000166035.26217.61.

Geithner, Julia, Felix Schneider, Zhong Wang, Julia Berneiser, Rosemarie Herzer, Christof Kessler, and Uwe Runge. 2012. "Predictors for Long-Term Seizure Outcome in Juvenile Myoclonic Epilepsy: 25-63 Years of Follow-Up." Epilepsia 53 (8): 1379-86. https://doi.org/10.1111/j.1528-1167.2012.03526.x.

Gennaro, G Di, A Picardi, A Sparano, A Mascia, G N Meldolesi, L G Grammaldo, V Esposito, and P P Quarato. 2012. "\{S\}eizure Clusters and Adverse Events during Pre-Surgical Video-\{E\}\{E\}\{G\} Monitoring with a Slow Anti-Epileptic Drug (\{A\}\{E\}\{D\}) Taper." Clin Neurophysiol 123 (3): 486-88.

Gherpelli, JLD, F Kok, S dal Forno, LC Elkis, BHW Lefevre, and AJ Diament. 1992. “Discontinuing Medication in Epileptic Children: A Study of Risk Factors Related to Recurrence." Epilepsia 33 (4): 681-86.

Giussani, Giorgia, Elisa Bianchi, Valentina Canelli, Giuseppe Erba, Carlotta Franchi, Alessandro Nobili, Josemir W Sander, and Ettore Beghi. 2017. "Antiepileptic Drug Discontinuation by People with Epilepsy in the General Population." Epilepsia 58 (9): 1524-32. https://doi.org/10.1111/epi.13853.

Glauser, Tracy, Elinor Ben-Menachem, Blaise Bourgeois, Avital Cnaan, David Chadwick, Carlos Guerreiro, Reetta Kalviainen, Richard Mattson, Emilio Perucca, and Torbjörn Tomson. 2006. "ILAE Treatment Guidelines: Evidence-Based Analysis of Antiepileptic Drug Efficacy and Effectiveness as Initial Monotherapy for Epileptic Seizures and Syndromes." Epilepsia 47 (7): 1094-1120. https://doi. org/10.1111/j.1528-1167.2006.00585.x.

Glauser, Tracy, Elinor Ben-Menachem, Blaise Bourgeois, Avital Cnaan, Carlos Guerreiro, Reetta Kalviainen, Richard Mattson, Jacqueline A French, Emilio Perucca, and Torbjörn Tomson. 2013. “Updated ILAE Evidence Review of Antiepileptic Drug Efficacy and Effectiveness as Initial Monotherapy for Epileptic Seizures and Syndromes." Epilepsia 54 (3): 551-63. https://doi.org/10.1111/epi.12074.

Gong, Jingjing, Yan Zhang, Zheng Yang, Yonghua Huang, Jun Feng, and Weiwei Zhang. 2013. “The Framing Effect in Medical Decision-Making: A Review of the Literature." Psychology, Health \& Medicine 18 (6): 645-53. https://doi.org/10.1080/13548506.2013.766352.

Gowers, William Richard. 1881. Epilepsy and Other Chronic Convulsive Diseases: Their Causes, Symptoms, and Treatment. London, United Kingdom: J \& A Churchill.

Gracia, Camilo Garcia, Ruta Yardi, Michael W Kattan, Dileep Nair, Ajay Gupta, William Bingaman, Jorge Gonzalez-martinez, and Lara Jehi. 2015. "Seizure Freedom Score: A New Simple Method to Predict Success of Epilepsy Surgery." Epilepsia 56 (3): 359-65. https://doi.org/10.1111/epi.12892.

Grambsch, Patricia M, and Therry M Therneau. 1994. "Proportional Hazards Tests and Diagnostics Based on Weighted Residuals." Biometrika 81 (3): 515-26.

Greenwood, Robert S., and Michael B. Tennison. 1999. "When to Start and Stop Anticonvulsant Therapy in Children." Arch. Neurol. 56: 1073-77.

Griffin, Craigan T, Mary E Abastillas, Carmel Armon, Julie Lacanlale, Boleslaw H Liwnicz, George Kaptain, and Lloyd A Dayes. 2004. "Early Antiepileptic Drug Reduction Following Anterior Temporal Lobectomy for Medically Intractable Complex Partial Epilepsy." Seizure 13: 434-37. https://doi. org/10.1016/j.seizure.2003.10.004.

Hader, Walter J, Jose Tellez-Zenteno, Amy Metcalfe, Lisbeth Hernandez-Ronquillo, Samuel Wiebe, ChurlSu Kwon, and Nathalie Jette. 2013. "Complications of Epilepsy Surgery: A Systematic Review of Focal Surgical Resections and Invasive EEG Monitoring." Epilepsia 54 (5): 840-47. https://doi. 
org/10.1111/epi.12161.

Hallböök, T, P Tideman, I Rosén, J Lundgren, and E Tideman. 2013. “Epilepsy Surgery in Children with Drug-Resistant Epilepsy, a Long-Term Follow-Up." Acta Neurologica Scandinavica 128: 414-21. https://doi.org/10.1111/ane.12154.

Harrell Jr, Frank E, Kerry L Lee, and Daniel B Mark. 1996. "Tutorial in Biostatistics - Multivariable Prognostic Models: Issues in Developing Models, Evaluating Assumptions and Adequacy, and Measureing and Reducing Errors." Statistics in Medicine 15: 361-87.

Hayden, Jill A, Pierre Coté, and Clarie Bombardier. 2006. "Evaluation of the Quality of Prognosis Studies in Systematic Reviews." Annals of Internal Medicine 144 (6): 427-38. http://www.annals.org/ content/144/6/427.short.

Healy, Liam, Maria Moran, Sumeet Singhal, Michael F O Donoghue, Rania Alzoubidi, and William P Whitehouse. 2018. “Relapse after Treatment Withdrawal of Antiepileptic Drugs for Juvenile Absence Epilepsy and Juvenile Myoclonic Epilepsy." Seizure 59: 116-22. https://doi.org/10.1016/j. seizure.2018.05.015.

Hedegärd, Emelie, Johan Bjellvi, Anna Edelvik, Bertil Rydenhag, Roland Flink, and Kristina Malmgren. 2014. "Complications to Invasive Epilepsy Surgery Workup with Subdural and Depth Electrodes:

A Prospective Population-Based Observational Study." Journal of Neurology, Neurosurgery \& Psychiatry 85: 716-20. https://doi.org/10.1136/jnnp-2013-306465.

Helbig, Ingo, Erin L Heinzen, and Heather C Mefford. 2016. “Primer Part 1 - The Building Blocks of Epilepsy Genetics." Epilepsia 57 (6): 861-68. https://doi.org/10.1111/epi.13381.

Helmstaedter, C, TW May, M Von Lehe, M Pfaefflin, A Ebner, HW Pannek, CE Elger, H Stefan, and J Schramm. 2014. "Temporal Lobe Surgery in Germany from 1988 to 2008: Diverse Trends in Etiological Subgroups." Eur. J. Neurol. 21 (6): 827-34. https://doi.org/10.1111/ene.12322.

Helmstaedter, C, K Schoof, T Rossmann, G Reuner, A Karlmeier, and G Kurlemann. 2010. "Introduction and First Validation of EpiTrack Junior, a Screening Tool for the Assessment of Cognitive Side Effects of Antiepileptic Medication on Attention and Executive Functions in Children and Adolescents with Epilepsy." Epilepsy \& Behavior 19: 55-64. https://doi.org/10.1016/j.yebeh.2010.06.042.

Helmstaedter, Christoph, Christian E Elger, and Juri Alexander Witt. 2016. "The Effect of Quantitative and Qualitative Antiepileptic Drug Changes on Cognitive Recovery after Epilepsy Surgery." Seizure 36: 63-69. https://doi.org/10.1016/j.seizure.2016.02.001.

Hemb, M, TR Velasco, MS Parnes, JY Wu, JT Lerner, JH Matsumoto, S Yudovin, et al. 2010. “Improved Outcomes in Pediatric Epilepsy Surgery: The UCLA Experience, 1986-2008." Neurology 74: 1768-75.

Hessen, Erik, M I Lossius, and Leif Gjerstad. 2011. "Improvement in Verbal Memory after Withdrawal of Carbamazepine and Valproate in Patients with Well-Controlled Epilepsy: A Randomized, DoubleBlind Study." Acta Neurol. Scand. 123: 385-89. https://doi.org/10.1111/j.1600-0404.2010.01452.x.

Hessen, Erik, MI Lossius, and Leif Gjerstad. 2009. "Antiepileptic Monotherapy Significantly Impairs Normative Scores on Common Tests of Executive Functions." Acta Neurol. Scand. 119: 194-98. https://doi.org/10.1111/j.1600-0404.2008.01109.x.

Hessen, Erik, Morten I Lossius, Ivar Reinvang, and Leif Gjerstad. 2006. "Influence of Major Antiepileptic Drugs on Attention, Reaction Time, and Speed of Information Processing: Results from a Randomized, Double-Blind, Placebo-Controlled Withdrawal Study of Seizure-Free Epilepsy Patients Receiving Monotherapy." Epilepsia 47 (12): 2038-45. https://doi.org/10.1111/j.15281167.2006.00805.x.

Higgins, Julian P T, and Simon G Thompson. 2002. "Quantifying Heterogeneity in a Meta-Analysis." Statistics in Medicine 21: 1539-58. https://doi.org/10.1002/sim.1186.

Höfler, Julia, Iris Unterberger, Judith Dobesberger, Giorgi Kuchukhidze, Gerald Walser, and Eugen Trinka. 2014. "Seizure Outcome in 175 Patients with Juvenile Myoclonic Epilepsy - A LongTerm Observational Study." Epilepsy Research 108 (10): 1817-24. https://doi.org/10.1016/j. eplepsyres.2014.09.008.

Holmes, Gregory L. 2016. "Effect of Seizures on the Developing Brain and Cognition." Seminars in Pediatric Neurology 23 (2): 120-26. https://doi.org/10.1016/j.spen.2016.05.001.

Holowach-Thurston, Jean, Don L Thurston, Barbara B Hixon, and Amy J Keller. 1982. "Prognosis in Childhood Epilepsy: Additional Follow-up of 148 Children 15 to 23 Years after Withdrawal of Anticonvulsant Therapy." The New England Journal of Medicine 306 (14): 831-36. 
International League Against Epilepsy. 1993. "Guidelines for Epidemiologic Studies on Epilepsy." Epilepsia 34 (4): 592-96.

loannidis, John P A. 2005. “Why Most Published Research Findings Are False” 2 (8). https://doi. org/10.1371/journal.pmed.0020124.

loannidis, John P A. 2014. “How to Make More Published Research True." PLoS Med 11 (10): e1001747. https://doi.org/10.1371/journal.pmed.1001747.

Jacoby, Ann, Gus A Baker, David Chadwick, and Anthony Johnson. 1993. “The Impact of Counselling with a Practical Statistical Model on Patients' Decision-Making about Treatment for Epilepsy: Findings from a Pilot Study." Epilepsy Research 16: 207-14.

Jacoby, Ann, Anthony Johnson, and David Chadwick. 1992."Psychosocial Outcomes of Antiepileptic Drug Discontinuation.pdf." Epilepsia 33 (6): 1123-31.

Janszky, J, I Janszky, R Schulz, M Hoppe, F Behne, H W Pannek, and A Ebner. 2005. “Temporal Lobe Epilepsy with Hippocampal Sclerosis: Predictors for Long-Term Surgical Outcome." Brain 128: 395-404. https://doi.org/10.1093/brain/awh358.

Jehi, Lara, Ruta Yardi, Kevin Chagin, Laura Tassi, Giorgio Lo Russo, Gregory Worrell, Wei Hu, et al. 2015. "Development and Validation of Nomograms to Provide Individualised Predictions of Seizure Outcomes after Epilepsy Surgery: A Retrospective Analysis." The Lancet Neurology 14 (3): 283-90. https://doi.org/10.1016/S1474-4422(14)70325-4.

Jobst, Barbara C, and Gregory D Cascino. 2019. "Resective Epilepsy Surgery for Drug-Resistant Focal Epilepsy: A Review." JAMA 313 (3): 285-93. https://doi.org/10.1001/jama.2014.17426.

Kaindl, A M, S Asimiadou, D Manthey, L Turski, and C Ikonomidou. 2006. "Antiepileptic Drugs and the Developing Brain." Cell Mol Life Sci 63: 399-413. https://doi.org/10.1007/s00018-005-5348-0.

Karnebeek, Clara D M Van, Sylvia A Tiebout, Jikkemien Niermeijer, Bwee Tien Poll-The, Aisha Ghani, Jost Wigand Richter, Hans Juergen Christen, Renata Gallagher, Hans Hartmann, and Sylvia StocklerIpsiroglu. 2016. “Pyridoxine-Dependent Epilepsy: An Expanding Clinical Spectrum." Pediatric Neurology 59: 6-12. https://doi.org/10.1016/j.pediatrneurol.2015.12.013.

Kerkhof, M, JAF Koekkoek, MJ Vos, MJ van den Bent, W Taal, TJ Postma, JEC Bromberg, et al. 2019. "Withdrawal of Antiepileptic Drugs in Patients with Low Grade and Anaplastic Glioma after Long-Term Seizure Freedom: A Prospective Observational Study." Journal of Neuro-Oncology 142: 463-70. https://doi.org/10.1007/s11060-019-03117-y.

Kerling, Frank, Elisabeth Pauli, Bogdan Lorber, Ingmar Blümcke, Michael Buchfelder, and Hermann Stefan. 2009. “Drug Withdrawal after Successful Epilepsy Surgery: How Safe Is It?" Epilepsy and Behavior 15 (4): 476-80. https://doi.org/10.1016/j.yebeh.2009.05.016.

Kilinç, S., and C. Campbell. 2008. "The Experience of Discontinuing Antiepileptic Drug Treatment: An Exploratory Investigation." Seizure 17 (6): 505-13. https://doi.org/10.1016/j.seizure.2008.01.002.

Kim, Eun-hee, and Tae-sung Ko. 2016. “Cognitive Impairment in Childhood Onset Epilepsy: Up-to-Date Information about Its Causes." Korean J Pediatr 59 (4): 155-64.

Kim, Young Dae, Kyoung Heo, Soo Chul Park, Kyoon Huh, Jin Woo Chang, Joong Uhn, Sang Sup Chung, and Byung In Lee. 2005. "Antiepileptic Drug Withdrawal after Successful Surgery for Intractable Temporal Lobe Epilepsy." Epilepsia 46 (2): 251-57.

Knapp, Guido, and Joachim Hartung. 2003. "Improved Tests for a Random Effects Meta-Regression with a Single Covariate." Statistics in Medicine 2710: 2693-2710. https://doi.org/10.1002/sim.1482.

Kobulashvili, Teia, Julia Höfler, Judith Dobesberger, Florian Ernst, Philippe Ryvlin, J Helen Cross, Kees P J Braun, et al. 2016. “Current Practices in Long-Term Video-EEG Monitoring Services: A Survey among Partners of the E-PILEPSY Pilot Network of Reference for Refractory Epilepsy and Epilepsy Surgery." Seizure 38: 38-45. https://doi.org/10.1016/j.seizure.2016.03.009.

Koch, S, K Titze, RB Zimmermann, M Schröder, U Lehmkuhl, and H Rauh. 1999. “Long-term Neuropsychological Consequences of Maternal Epilepsy and Anticonvulsant Treatment during Pregnancy for School-age Children and Adolescents." Epilepsia 40 (9): 1237-43.

Korkman, Marit, Pekka Lahti-Nuuttila, Marja Laasonen, Sally L Kemp, and James Holdnack. 2013. "Neurocognitive Development in 5- to 16-Year- Old North American Children : A Cross-Sectional Study American Children : A Cross-Sectional Study." Child Neuropsychology 19 (5): 516-39. https:// doi.org/10.1080/09297049.2012.705822.

Kovac, Stjepana, Vejay N Vakharia, Catherine Scott, and Beate Diehl. 2017. "Invasive Epilepsy Surgery 
Evaluation." Seizure: European Journal of Epilepsy 44: 125-36. https://doi.org/10.1016/j. seizure.2016.10.016.

Krsek, Pavel, Tom Pieper, Anja Karlmeier, Michelle Hildebrandt, Dieter Kolodziejczyk, Peter Winkler, Elisabeth Pauli, Ingmar Blümcke, and Hans Holthausen. 2009. "Different Presurgical Characteristics and Seizure Outcomes in Children with Focal Cortical Dysplasia Type I or II." Epilepsia 50 (1): 12537. https://doi.org/10.1111/j.1528-1167.2008.01682.x.

Kumar, Atul, Antonio Valentín, Danish Humayon, Alix L Longbottom, Diego Jimenez-Jimenez, Nandini Mullatti, Robert CD Elwes, et al. 2013. “Preoperative Estimation of Seizure Control after Resective Surgery for the Treatment of Epilepsy." Seizure 22: 818-26. https://doi.org/10.1016/j. seizure.2013.06.010.

Kumlien, Eva, and Peter Mattsson. 2010. "Attitudes towards Epilepsy Surgery: A Nationwide Survey among Swedish Neurologists." Seizure: European Journal of Epilepsy 19 (4): 253-55. https://doi. org/10.1016/j.seizure.2010.02.001.

Kwan, P, and Martin J Brodie. 2000. "Early Identification of Refractory Epilepsy." The New England Journal of Medicine 342: 314-19. https://doi.org/https://dx.doi.org/10.1056/NEJM200002033420503.

Kwan, Patrick, Alexis Arzimanoglou, Anne T Berg, Martin J Brodie, W Allen Hauser, Gary Mathern, Solomon L Moshé, Emilio Perucca, Samuel Wiebe, and Jacqueline A French. 2010. “Definition of Drug Resistant Epilepsy: Consensus Proposal by the Ad Hoc Task Force of the ILAE Commission on Therapeutic Strategies." Epilepsia 51 (6): 1069-77. https://doi.org/10.1111/j.15281167.2009.02397.x.

Lachhwani, Deepak K. 2005. "Pediatric Epilepsy Surgery: Lessons and Challenges." Seminars in Pediatric Neurology 12: 114-18. https://doi.org/10.1016/j.spen.2005.04.001.

Lachhwani, Deepak K, Tobias Loddenkemper, Katherine D Holland, Prakash Kotagal, Edward Mascha, William Bingaman, and Elaine Wyllie. 2008. "Discontinuation of Medications after Successful Epilepsy Surgery in Children." Pediatric Neurology 38: 340-44. https://doi.org/10.1016/j. pediatrneurol.2008.01.010.

Ladino, Lady D, Lizbeth Hernández-Ronquillo, and José F Téllez-Zenteno. 2014. “Management of Antiepileptic Drugs Following Epilepsy Surgery: A Meta-Analysis." Epilepsy Research 108 (4): 765 74. https://doi.org/10.1016/j.eplepsyres.2014.01.024.

Lagae, Lieven. 2006. "Cognitive Side Effects of Anti-Epileptic Drugs The Relevance in Childhood Epilepsy." Seizure 15: 235-41. https://doi.org/10.1016/j.seizure.2006.02.013.

Lagae, Lieven. 2017. “The Importance of Assessing Behaviour and Cognition in Antiepileptic Drug Trials in Children and Adolescents." Acta Neurologica Belgica 117 (2): 425-32. https://doi.org/10.1007/ s13760-016-0734-y.

Lamberink, Herm J, Kim Boshuisen, P.C. Van Rijen, Peter H. Gosselaar, and Kees P J Braun. 2015. “Changing Profiles of Pediatric Epilepsy Surgery Candidates over Time: A Nationwide Single-Center Experience from 1990 to 2011." Epilepsia 56 (5): 717-25. https://doi.org/10.1111/epi.12974.

Lamberink, Herm J, W.M. Otte, Karin Geleijns, and Kees P J Braun. 2015. "Antiepileptic Drug Withdrawal in Medically and Surgically Treated Patients: A Meta-Analysis of Seizure Recurrence and Systematic Review of Its Predictors." Epileptic Disorders 17 (3).

Lamberink, Herm J, Willem M Otte, Ada T Geerts, Milen Pavlovic, Julio Ramos-Lizana, Anthony G Marson, Jan Overweg, et al. 2017."Individualised Prediction Model of Seizure Recurrence and Long-Term Outcomes after Withdrawal of Antiepileptic Drugs in Seizure-Free Patients: A Systematic Review and Individual Participant Data Meta-Analysis." The Lancet Neurology 16 (7): 523-531, http://dx.doi. org/10.1016/S1474-4422(17)30114-X.

Lamdhade, SJ, and GM Taori. 2002. "Study of Factors Responsible for Recurrence of Seizures in Controlled Epileptics for More than 1 Years after Withdrawal of Antiepileptic Drugs." Neurol India 50: 295-300.

Lancaster, Eric, and Josep Dalmau. 2012. "Neuronal Autoantigens - Pathogenesis, Associated Disorders and Antibody Testing." Nature Reviews Neurology 8 (7): 380-90. https://doi.org/10.1038/ nrneurol.2012.99.

Lee, Seo-Young, Ji-Young Lee, Dong Wook Kim, Sang Kun Lee, and Chun Kee Chung. 2008. "Factors Related to Successful Antiepileptic Drug Withdrawal after Anterior Temporal Lobectomy for Medial Temporal Lobe Epilepsy." Seizure: The Journal of the British Epilepsy Association 17 (1): 11-18. https://doi.org/10.1016/j.seizure.2007.05.014. 
Lettori, D, D Battaglia, A Sacco, C Veredice, D Chieffo, L Massimi, T Tartaglione, et al. 2008. “Early Hemispherectomy in Catastrophic Epilepsy - a Neuro-Cognitive and Epileptic Long-Term FollowUp." Seizure 17: 49-63. https://doi.org/10.1016/j.seizure.2007.06.006.

Levitt, Pat, John A Harvey, Eitan Friedman, Kenny Simansky, and E Hazel Murphy. 1997. “New Evidence for Neurotransmitter Influences on Brain Development." Trends Neurosci 20 (6): 269-74.

Li, Wei, Yang Si, Xue-mei Zou, Dong-mei An, Hui Yang, and Dong Zhou. 2014. "Prospective Study on the Withdrawal and Reinstitution of Antiepileptic Drugs among Seizure-Free Patients in West China." Journal of Clinical Neuroscience 21: 997-1001. https://doi.org/10.1016/j.jocn.2013.09.019.

Loring, David W, and Kimford J Meador. 2004. "Cognitive Side Effects of Antiepileptic Drugs in Children." Neurology 62: 872-77.

Lossius, Morten Ingvar, Erik Hessen, Petter Mowinckel, Knut Stavem, Jan Erikssen, Paal Gulbrandsen, and Leif Gjerstad. 2008. "Consequences of Antiepileptic Drug Withdrawal: A Randomized, Double-Blind Study (Akershus Study)." Epilepsia 49 (3): 455-63. https://doi.org/10.1111/j.15281167.2007.01323.x.

Marcus, JC. 1998. "Stopping Antiepileptic Therapy in Mentally Retarded, Epileptic Children." Neuropediatrics 29: 26-28.

Marinas, A, E Elices, A Gil-Nagel, J Salas-Puig, J C Sánchez, M Carreño, V Villanueva, J Rosendo, J Porcel, and JM Serratosa. 2011. "Socio-Occupational and Employment Profile of Patients with Epilepsy." Epilepsy \& Behavior 21 (3): 223-27. https://doi.org/10.1016/j.yebeh.2011.01.025.

Marshall, Andrea, Douglas G Altman, Patrick Royston, and Roger L Holder. 2010. “Comparison of Techniques for Handling Missing Covariate Data within Prognostic Modelling Studies: A Simulation Study." BMC Medical Research Methodology 10 (7): 1-16. https://doi.org/10.1186/14712288-10-7.

Martin-McGill, KJ, CF Jackson, R Bresnahan, RG Levy, and PN Cooper. 2018. “Ketogenic Diets for DrugResistant Epilepsy." Cochrane Database of Systematic Reviews Issue 11: CD001903. https://doi. org/10.1002/14651858.CD001903.pub4.

Matricardi, M, M Brinciotti, and P Benedetti. 1989. “Outcome after Discontinuation of Antiepileptic Drug Therapy in Children with Epilepsy." Epilepsia 30 (5): 582-89.

Meador, KJ. 2002. "Cognitive Outcomes and Predictive Factors in Epilepsy." Neurology 58 (8 Suppl 5): $\mathrm{S} 21-26$.

Medical Research Council, Antiepileptic Drug Withdrawal Study Group. 1993. “Prognostic Index for Recurrence of Seizures after Remission Ofepilepsy." BMJ 306: 1374-78.

Meekes, Joost, Olga Braams, Kees P J Braun, Aag Jennekens-Schinkel, and Onno van Nieuwenhuizen. 2013. "Verbal Memory after Epilepsy Surgery in Childhood." Epilepsy Research 107: 146-55. https:// doi.org/10.1016/j.eplepsyres.2013.08.017.

Mehta, V, CD Ferrie, J Helen Cross, and G Vadlamani. 2015. "Corticosteroids Including ACTH for Childhood Epilepsy Other than Epileptic Spasms." Cochrane Database of Systematic Reviews Issue 6: CD005222. https://doi.org/10.1002/14651858.CD005222.pub3.

Menon, Ramsekhar, Chaturbhuj Rathore, Sankara P. Sarma, and Kurupath Radhakrishnan. 2012. "Feasibility of Antiepileptic Drug Withdrawal Following Extratemporal Resective Epilepsy Surgery." Neurology 79 (8): 770-76. https://doi.org/10.1212/WNL.0b013e3182644f7d.

Mohanraj, Rajiv, John Norrie, Linda J Stephen, Kevin Kelly, Nikolas Hitiris, and Martin J Brodie. 2006. "Mortality in Adults with Newly Diagnosed and Chronic Epilepsy: A Retrospective Comparative Study." Lancet Neurology 5: 481-87. https://doi.org/10.1016/S1474-4422(06)70448-3.

MPS Research Unit. 2000. “Pest 4: Operating Manual.” University of Reading.

MRC AED withdrawal study group. 1991."Randomised Study of Antiepileptic Drug Withdrawal in Patients in Remission." Lancet 337 (8751): 1175-80.

Mula, Marco, and Michael R Trimble. 2009. "Antiepileptic Drug-Induced Cognitive Adverse Effects: Potential Mechanisms and Contributing Factors." CNS Drugs 23 (2): 121-37.

Munckhof, Bart van den, Violet van Dee, Liora Sagi, Roberto H Caraballo, Pierangelo Veggiotti, Elina Liukkonen, Tobias Loddenkemper, et al. 2015. “Treatment of Electrical Status Epilepticus in Sleep: A Pooled Analysis of 575 Cases." Epilepsia 56 (11): 1738-46. https://doi.org/10.1111/epi.13128.

Murro, Anthony M, Don W King, JR Smith, Brian B Gallagher, HF Flanigin, David W Loring, Gregory P Lee, R Campbell, K Meador, and H Revolinski. 1991. “Withdrawal of Antiepileptic Medications Following 
Epilepsy Surgery." J Epilepsy 4: 39-42.

Nederlandse Vereniging voor Neurologie. 2015. “Richtlijnen Epilepsie: Neuropsychologisch Onderzoek. Accessed on 02/28/2019." 2015. http://epilepsie.neurologie.nl/cmssite/index.php?pageid=324.

Ngugi, Anthony K, Christian Bottomley, Immo Kleinschmidt, Josemir W Sander, and Charles R Newton. 2010. "Estimation of the Burden of Active and Life-Time Epilepsy: A Meta-Analytic Approach." Epilepsia 51 (5): 883-90. https://doi.org/10.1111/j.1528-1167.2009.02481.x.

Nguyen, L, JM Rigo, V Rocher, S Belachew, Brigitte Malgrange, Bernard Rogister, P Leprince, and G Moonen. 2001. "Neurotransmitters as Early Signals for Central Nervous System Development." Cell Tissue Res 205: 187-202. https://doi.org/10.1007/s004410000343.

O'Callaghan, Finbar J K, Stuart W Edwards, Fabienne Dietrich Alber, Eleanor Hancock, Anthony L Johnson, Colin R Kennedy, Marcus Likeman, et al. 2017. "Safety and Eff Ectiveness of Hormonal Treatment versus Hormonal Treatment with Vigabatrin for Infantile Spasms (ICISS): A Randomised , Multicentre, Open-Label Trial." The Lancet Neurology 16 (1): 33-42. https://doi.org/10.1016/ S1474-4422(16)30294-0.

Ohta, Hodaka, Yoko Ohtsuka, Toshihide Tsuda, and Eiji Oka. 2004. "Prognosis after Withdrawal of Antiepileptic Drugs in Childhood-Onset Cryptogenic Localization-Related Epilepsies." Brain and Development 26: 19-25. https://doi.org/10.1016/S0387-7604(03)00089-5.

Oijen, Marieke van, Hanneke de Waal, Peter C van Rijen, Aag Jennekens-Schinkel, Alexander C van Huffelen, and Onno van Nieuwenhuizen. 2006. "Resective Epilepsy Surgery in Childhood: The Dutch Experience 1992-2002." European Journal of Paediatric Neurology 10: 114-23. https://doi. org/10.1016/j.ejpn.2006.04.003.

Olmez, Akgun, Umut Arslan, Guzide Turanli, and Sabiha Aysun. 2009. "Risk of Recurrence after Drug Withdrawal in Childhood Epilepsy." Seizure 18: 251-56. https://doi.org/10.1016/j. seizure.2008.10.011.

Operto, Francesca Felicia, Roberta Mazza, Grazia Maria Giovanna Pastorino, Stella Campanozzi, Lucia Margari, and Giangennaro Coppola. 2019. "Parental Stress in Pediatric Epilepsy after Therapy Withdrawal." Epilepsy \& Behavior 94: 239-42. https://doi.org/10.1016/j.yebeh.2019.03.029.

Ou, Shuchun, Lu Xia, Rong Li, Li Wang, Li Xia, Qin Zhou, and Songqing Pan. 2018. "Long-Term Outcome of Seizure-Free Patients and Risk Factors of Relapse Following Antiepileptic Drug Withdrawal." Epilepsy \& Behavior 88: 295-300. https://doi.org/10.1016/j.yebeh.2018.09.028.

Overweg, J., C D Binnie, J. Oosting, and A. J. Rowan. 1987. "Clinical and EEG Prediction of Seizure Recurrence Following Antiepileptic Drug Withdrawal." Epilepsy Research 1 (5): 272-83. https://doi. org/10.1016/0920-1211(87)90002-7.

Ovsiew, F. 2004. "Antiepileptic Drugs in Psychiatry." Journal of Neurology, Neurosurgery \& Psychiatry 75 (12): 1655-58.

Panebianco, M, A Rigby, J Weston, and Anthony G Marson. 2015. “Vagus Nerve Stimulation for Partial Seizures." Cochrane Database of Systematic Reviews Issue 4: CD002896. https://doi. org/10.1002/14651858.CD002896.pub2.

Park, Kyung Il, Sang Kun Lee, Kon Chu, Keun Hwa Jung, Eun Kee Bae, Jin Soo Kim, Jung Ju Lee, Seo Young Lee, and Chun Kee Chung. 2010. "Withdrawal of Antiepileptic Drugs after Neocortical Epilepsy Surgery." Annals of Neurology 67 (2): 230-38. https://doi.org/10.1002/ana.21884.

Pavlović, Milen, Nebojša Jović, and Tatjana Pekmezović. 2012. “Withdrawal of Antiepileptic Drugs in Young Patients with Cryptogenic Focal Epilepsies." Seizure 21 (6): 431-36. https://doi. org/10.1016/j.seizure.2012.04.008.

Perucca, Piero, Jewell Carter, Victoria Vahle, and Frank G Gilliam. 2009. "Adverse Antiepileptic Drug Effects: Toward a Clinically and Neurobiologically Relevant Taxonomy." Neurology 72 (14): 1223-29. https://doi.org/10.1212/01.wnl.0000345667.45642.61.

Perucca, Piero, and Frank G Gilliam. 2012. "Adverse Eff Ects of Antiepileptic Drugs." Lancet Neurology 11: 792-802. https://doi.org/10.1016/S1474-4422(12)70153-9.

Pestana-Knight, Elia M, Nicholas K Schiltz, Paul M Bakaki, Siran M Koroukian, Samden D Lhatoo, and Kitti Kaiboriboon. 2015. "Increasing Utilization of Pediatric Epilepsy Surgery in the United States between 1997 and 2009." Epilepsia 56 (3): 375-81. https://doi.org/10.1111/epi.12912.

Phal, PM, Alexander Usmanov, Gary M Nesbit, James C Anderson, David Spencer, Paul Wang, Jonathan A Helwig, Colin Roberts, and Bronwyn E Hamilton. 2008. "Qualitative Comparison of 3-T and 1.5-T 
MRI in the Evaluation of Epilepsy." American Journal of Roentgenology 191 (3): 890-95. https://doi. org/10.2214/AJR.07.3933.

Phung, Jennifer, Gary W Mathern, and Paul Krogstad. 2013. "Timing and Predictors of Fever and Infection after Craniotomy for Epilepsy in Children." The Pediatric Infectious Disease Journal 32 (5): 450-59. https://doi.org/10.1097/INF.0b013e318287b408.

Picot, Marie-Christine, Michel Baldy-Moulinier, Jean-Pierre Daurès, Pierre Dujols, and Arielle Crespel. 2008. "The Prevalence of Epilepsy and Pharmacoresistant Epilepsy in Adults: A Population-Based Study in a Western European Country." Epilepsia 49 (7): 1230-38. https://doi.org/10.1111/j.15281167.2008.01579.x.

R Core Team. 2014. “R: A Language and Environment for Statistical Computing." Vienna, Austria: R Foundation for Statistical Computing.

Radhakrishnan, Ashalatha, Ramshekhar Menon, Sanjeev V Thomas, Mathew Abraham, George Vilanilam, Chandrashekharan Kesavadas, Bejoy Thomas, Ajith Cherian, and Prasad Ravi Varma. 2018. "' Time Is Brain '- How Early Should Surgery Be Done in Drug-Resistant TLE?" Acta Neurol. Scand. 138: 531-40. https://doi.org/10.1111/ane.13008.

Ramos-Lizana, J, J Aguirre-Rodríguez, P Aguilera-López, and E Cassinello-García. 2010. “Recurrence Risk after Withdrawal of Antiepileptic Drugs in Children with Epilepsy: A Prospective Study." European Journal of Paediatric Neurology: EJPN: Official Journal of the European Paediatric Neurology Society 14 (2): 116-24. https://doi.org/10.1016/j.ejpn.2009.05.006.

Rathore, Chaturbhuj, Malcolm K Jeyaraj, Gopal K Dash, Pandurang Wattamwar, Neeraj Baheti, Sankara P Sarma, and Kurupath Radhakrishnan. 2018. "Outcome after Seizure Recurrence on Antiepileptic Drug Withdrawal Following Temporal Lobectomy." Neurology 91 (3): e208-16. https://doi. org/10.1212/WNL.0000000000005820.

Rathore, Chaturbhuj, Samhita Panda, P. Sankara Sarma, and Kurupath Radhakrishnan. 2011. “How Safe Is It to Withdraw Antiepileptic Drugs Following Successful Surgery for Mesial Temporal Lobe Epilepsy?" Epilepsia 52 (3): 627-35. https://doi.org/10.1111/j.1528-1167.2010.02890.x.

Reilly, Colin, Patricia Atkinson, Krishna B Das, Richard F M Chin, Sarah E Aylett, Victoria Burch, Christopher Gillberg, Rod C Scott, and Brian G R Neville. 2015. “Cognition in School-Aged Children with 'Active 'Epilepsy: A Population-Based Study." Journal of Clinical and Experimental Neuropsychology 37 (4): 429-38.

Reinisch, JM, SA Sanders, EL Mortensen, and DB Rubin. 1995. “In Utero Exposure to Phenobarbital and Intelligence Deficits in Adult Men." JAMA 274 (19): 1518-25.

Retz, W, J Kornhuber, and P Riederer. 1996. "Neurotransmission and the Ontogeny of Human Brain." Journal of Neural Transmission 103: 403-19.

Rheims, Sylvain, and Philippe Ryvlin. 2014."Patients' Safety in the Epilepsy Monitoring Unit: Time for Revising Practices." Curr Opin Neurol 27 (2): 213-18. https://doi.org/10.1097/ WCO.0000000000000076.

Riley, Richard D, Paul C Lambert, and Ghada Abo-Zaid. 2010. "Meta-Analysis of Individual Participant Data: Rationale, Conduct, and Reporting." BMJ (Clinical Research Ed.) 340: c221. https://doi. org/10.1136/bmj.c221.

Rogawski, Michael A, and Wolfgang Löscher. 2004. "The Neurobiology of Antiepileptic Drugs." Nature Reviews Neuroscience 5: 553-64. https://doi.org/10.1038/nrn1430.

Rubboli, Guido, Sandor Beniczky, Steven Claus, Maria Paola, Philippe Kahane, Hermann Stefan, Walter Van Emde, et al. 2015. "A European Survey on Current Practices in Epilepsy Monitoring Units and Implications for Patients' Safety." Epilepsy \& Behavior 44: 179-84. https://doi.org/10.1016/j. yebeh.2015.02.004.

Rubboli, Guido, Anne Sabers, Peter Uldall, and Kristina Malmgren. 2017. “Management of Antiepileptic Treatment after Epilepsy Surgery - Practices and Problems." Current Pharmaceutical Design 23: 5749-59. https://doi.org/10.2174/1381612823666170823111712.

Russ, Shirley A, Kandyce Larson, and Neal Halfon. 2012. "A National Profile of Childhood Epilepsy and Seizure Disorder." Pediatrics 129 (2): 256-64. https://doi.org/10.1542/peds.2010-1371.

Ryvlin, Philippe, J Helen Cross, and Sylvain Rheims. 2014. "Epilepsy Surgery in Children and Adults." The Lancet Neurology 13 (11): 1114-26. https://doi.org/10.1016/S1474-4422(14)70156-5.

Ryvlin, Philippe, M Cucherat, and Sylvain Rheims. 2011."Risk of Sudden Unexpected Death in Epilepsy 
in Patients given Adjunctive Antiepileptic Treatment for Refractory Seizures: A Meta-Analysis of Placebo-Controlled Randomised Trials." Lancet Neurology 10 (11): 961-68.

Ryvlin, Philippe, Lina Nashef, Samden D Lhatoo, Lisa M Bateman, Jonathan Bird, Andrew Bleasel, Paul Boon, et al. 2013. "Incidence and Mechanisms of Cardiorespiratory Arrests in Epilepsy Monitoring Units (MORTEMUS): A Retrospective Study." Lancet Neurology 12: 966-77. https://doi.org/10.1016/ S1474-4422(13)70214-X.

Schachter, Steven C. 2018. "Determining When to Stop Antiepileptic Drug Treatment." Curr Opin Neurol 31: 211-15. https://doi.org/10.1097/WCO.0000000000000530.

Scheffer, Ingrid E, S Berkovich, Giuseppe Capovilla, MB Connolly, Jacqueline A French, L Guilhoto, Edouard Hirsch, et al. 2017. "ILAE Classification of the Epilepsies- Position Paper of the ILAE Commission for Classification and Terminology." Epilepsia 58 (4): 512-21.

Schiller, Y, G Cascino, Elson L So, and WR Marsh. 2000. "Discontinuation of Antiepileptic Drugs after Successful Epilepsy Surgery." Neurology 54 (2): 346-49.

Schiltz, Nicholas K, Siran M Koroukian, Samden D Lhatoo, and Kitti Kaiboriboon. 2013. "Temporal Trends in Pre-Surgical Evaluations and Epilepsy Surgery in the U. S. from 1998 to 2009." Epilepsy Research 103: 270-78. https://doi.org/10.1016/j.eplepsyres.2012.07.016.

Schmidt, Dieter. 2011. "AED Discontinuation May Be Dangerous for Seizure-Free Patients." Journal of Neural Transmission 118 (2): 183-86. https://doi.org/10.1007/s00702-010-0528-y.

Schmidt, Dieter, Christoph Baumgartner, and L Wolfgang. 2004. "The Chance of Cure Following Surgery for Drug-Resistant Temporal Lobe Epilepsy - What Do We Know and Do We Need to Revise Our Expectations?" Epilepsy Research 60: 187-201. https://doi.org/10.1016/j.eplepsyres.2004.07.004.

Schmidt, Dieter, and W. Löscher. 2005. “Uncontrolled Epilepsy Following Discontinuation of Antiepileptic Drugs in Seizure-Free Patients: A Review of Current Clinical Experience." Acta Neurologica Scandinavica 111 (5): 291-300. https://doi.org/10.1111/j.1600-0404.2005.00408.x.

Schooneveld, Monique M J Van, and Kees P J Braun. 2013. “Cognitive Outcome after Epilepsy Surgery in Children." Brain and Development 35 (8): 721-29. https://doi.org/10.1016/j.braindev.2013.01.011.

Schooneveld, Monique M J Van, Nicole Van Erp, Kim Boshuisen, Joost Meekes, and Kees P J Braun. 2013. "Withdrawal of Antiepileptic Drugs Improves Psychomotor Speed after Childhood Epilepsy Surgery." Epilepsy Research 107 (1-2): 200-203. https://doi.org/10.1016/j.eplepsyres.2013.08.004.

Schulze-Bonhage, Andreas. 2017. "Brain Stimulation as a Neuromodulatory Epilepsy Therapy." Seizure: European Journal of Epilepsy 44: 169-75. https://doi.org/10.1016/j.seizure.2016.10.026.

Schwenkhagen, Anneliese M, and Stefan R G Stodieck. 2008. "Which Contraception for Women with Epilepsy?" Seizure 17: 145-50. https://doi.org/10.1016/j.seizure.2007.11.013.

Scolnik, Dennis, Irena Nulman, Joanne Rovet, David Gladstone, Deborah Czuchta, H. Allen Gardner, Richard Gladstone, et al. 1994. "Neurodevelopment of Children Exposed in Utero to Phenytoin and Carbamazepine Monotherapy." JAMA 271 (10): 767-70. https://doi.org/10.1001/ jama.1994.03510340057034.

Serra, J G, M A Montenegro, and M M Guerreiro. 2005. "Antiepileptic Drug Withdrawal in Childhood: Does the Duration of Tapering off Matter for Seizure Recurrence?" J Child Neurol 20 (7): 624-26. https:// doi.org/10.1177/08830738050200071901.

Shields, WD. 2000. "Catastrophic Epilepsy in Childhood." Epilepsia 41: Supple 2:52-56.

Shih, Jerry J, and Juan G Ochoa. 2009. "A Systematic Review of Antiepileptic Drug Initiation and Withdrawal." The Neurologist 15 (3): 122-31. https://doi.org/10.1097/NRL.0b013e3181901ad3.

Shinnar, Shlomo, and Anne T Berg. 1995. "Withdrawal of Antiepileptic Drugs." Curr Opin Neurol 8: 103-6.

Shinnar, Shlomo, Anne T Berg, SL Moshé, H Kang, C O'Dell, M Alemany, ES Goldensonn, and WA Hauser. 1994. "Discontinuing Antiepileptic Drugs in Children with Epilepsy: A Prospective Study." Annals of Neurology 35: 534-45.

Shinnar, Shlomo, Eileen P Vining, E David Mellits, Bernard J D'Souza, Kenton R Holden, Rosemary A Baumgardner, and John M Freeman. 1985. “Discontinuing Antiepileptic Medication in Children with Epilepsy after Two Years without Seizures: A Prospective Study." The New England Journal of Medicine 313 (16): 976-80.

Shorvon, Simon D, and David M G Goodridge. 2013. "Longitudinal Cohort Studies of the Prognosis of Epilepsy : Contribution of the National General Practice Study of Epilepsy and Other Studies." Brain 136: 3497-3510. https://doi.org/10.1093/brain/awt223. 
Sillanpää, Matti, Leena Haataja, and Shlomo Shinnar. 2004. “Perceived Impact of Childhood-Onset Epilepsy on Quality of Life as an Adult." Epilepsia 45 (8): 971-77. https://doi.org/10.1111/j.00139580.2004.44203.x.

Sillanpää, Matti, and D Schmidt. 2006. "Prognosis of Seizure Recurrence after Stopping Antiepileptic Drugs in Seizure-Free Patients: A Long-Term Population-Based Study of Childhood-Onset Epilepsy." Epilepsy \& Behavior 8: 713-19. https://doi.org/10.1016/j.yebeh.2006.02.014.

Silva-Alves, Mariana S, Rodrigo Secolin, Benilton S Carvalho, Clarissa L Yasuda, Elizabeth Bilevicius, Marina K M Alvim, Renato O Santos, Claudia V Maurer-Morelli, Fernando Cendes, and Iscia Lopes-Cendes. 2017. "A Prediction Algorithm for Drug Response in Patients with Mesial Temporal Lobe Epilepsy Based on Clinical and Genetic Information." PLos One 12 (1): e0169214. https://doi.org/10.1371/ journal.pone.0169214.

Simasathien, Thitiwan, Sumeet Vadera, Imad Najm, Ajay Gupta, William Bingaman, and Lara Jehi. 2013. "Improved Outcomes with Earlier Surgery for Intractable Frontal Lobe Epilepsy." Annals of Neurology 73: 646-54. https://doi.org/10.1002/ana.23862.

Sinclair, D Barry, Laura Jurasek, Matt Wheatley, Anita Datta, Donald Gross, Nizam Ahmed, Daphne Quigley, Thomas Snyder, Keith Aronyk, and John Mckean. 2007. "Discontinuation of Antiepileptic Drugs after Pediatric Epilepsy Surgery." Pediatric Neurology 37: 200-202. https://doi.org/10.1016/j. pediatrneurol.2007.05.004.

Skirrow, C., J Helen Cross, F. Cormack, W. Harkness, F. Vargha-Khadem, and T. Baldeweg. 2011. “Long-Term Intellectual Outcome after Temporal Lobe Surgery in Childhood." Neurology 76 (15): 1330-37. https://doi.org/10.1212/WNL.0b013e31821527fo.

Skirrow, C, J Helen Cross, Rosie Owens, Louise Weiss-Croft, Patricia Martin-Sanfilippo, Tina Banks, Emily Shah, William Harkness, Faraneh Vargha-Khadem, and Torsten Baldeweg. 2019. “Determinants of IQ Outcome after Focal Epilepsy Surgery in Childhood: A Longitudinal Case-Control Neuroimaging Study." Epilepsia 60: 872-84. https://doi.org/10.1111/epi.14707.

So, Elson L, and Ricky W Lee. 2014. "Epilepsy Surgery in MRI-Negative Epilepsies." Curr Opin Neurol 27 (2): 206-12. https://doi.org/10.1097/WCO.0000000000000078.

Specchio, Luigi M, and Ettore Beghi. 2004. "Should Antiepileptic Drugs Be Withdrawn in Seizure-Free Patients?" CNS Drugs 18 (4): 201-12.

Specchio, Luigi M, L Tramacere, a La Neve, and E Beghi. 2002."Discontinuing Antiepileptic Drugs in Patients Who Are Seizure Free on Monotherapy." Journal of Neurology, Neurosurgery, and Psychiatry 72 (1): 22-25. https://doi.org/10.1136/jnnp.72.1.22.

Spencer, Susan, and Linda Huh. 2008. "Outcomes of Epilepsy Surgery in Adults and Children." Lancet Neurology 7: 525-37.

Stagi, Stefano, Stefania Lasorella, Alessandra Piccorossi, Giulia lapadre, Stefano Stagi, Stefania Lasorella, Alessandra Piccorossi, and Giulia lapadre. 2016. “Cessation of Epilepsy Therapy in Children." Expert Review of Neurotherapeutics 16 (5): 549-59. https://doi.org/10.1586/14737175.2016.1168296.

Steensberg, Alvilda T, Ane Sophie Olsen, Minna Litman, Bo Jespersen, Miriam Kolko, and Lars H Pinborg. 2018. “Visual Field Defects after Temporal Lobe Resection for Epilepsy." Seizure: European Journal of Epilepsy 54: 1-6. https://doi.org/10.1016/j.seizure.2017.11.011.

Steinbrenner, Mirja, Alexander B Kowski, and Martin Holtkamp. 2019. "Referral to Evaluation for Epilepsy Surgery : Reluctance by Epileptologists and Patients." Epilepsia ePub ahead. https://doi. org/10.1111/epi.14641.

Sterne, Jonathan A C, lan R White, John B Carlin, Michael Spratt, Patrick Royston, Michael G Kenward, Angela M Wood, and James R Carpenter. 2009. "Multiple Imputation for Missing Data in Epidemiological and Clinical Research: Potential and Pitfalls." BMJ 338: b2393. https://doi. org/10.1136/bmj.b2393.

Steyerberg, E, K G M Moons, D van der Windt, J Hayden, P Perel, S Schroter, R Riley, H Hemingway, and R B Altman. 2013. "Prognosis Research Strategy (PROGRESS) Series 3: Prognostic Model Research." PLoS Med 10 (2): e1001381. https://doi.org/10.1371/jour-.

Stockler, Sylvia, Barbara Plecko, Sidney M Gospe Jr, Marion Coulter-Mackie, Mary Connolly, Clara Van Karnebeek, Saadet Mercimek-Mahmutoglu, et al. 2011.“Pyridoxine Dependent Epilepsy and Antiquitin Deficiency - Clinical and Molecular Characteristics and Recommendations for Diagnosis, Treatment and Follow-Up." Molecular Genetics and Metabolism 104: 48-60. https://doi. 
org/10.1016/j.ymgme.2011.05.014.

Strozzi, I, SJ Nolan, MR Sperling, DM Wingerchuk, and J Sirven. 2015. “Early versus Late Antiepileptic Drug Withdrawal for People with Epilepsy in Remission (Review)." Cochrane Database of Systematic Reviews 2: CD001902.

Su, Lingying, Qing Di, Nian Yu, and Yanfang Zhang. 2013. “Predictors for Relapse after Antiepileptic Drug Withdrawal in Seizure-Free Patients with Epilepsy." Journal of Clinical Neuroscience 20: 790-94. https://doi.org/10.1016/j.jocn.2012.07.010.

Sulzbacher, Stephen, Jacqueline Farwell, Nancy Temkin, Ann S Lu, and Deborah G Hirtz. 1999. “Late Cognitive Effects of Early Treatment with Phenobarbital." Clinical Pediatrics 38 (7): 387-94.

Sutton, Alex J, Keith R Abrams, David R Jones, Trevor A Sheldon, and Fujian Song. 2000. Methods for MetaAnalysis in Medical Research. Chichester: John Wiley \& Sons, LTD.

Swisher, Christa B, and Saurabh R Sinha. 2013. "Survey of Current Practices among US Epileptologists of Antiepileptic Drug Withdrawal after Epilepsy Surgery." Epilepsy \& Behavior 26 (2): 203-6. https:// doi.org/10.1016/j.yebeh.2012.11.053.

Syvertsen, Marte, Ida Fløgstad, Ulla Enger, Cecilie Johannessen, and Jeanette Koht. 2019. "Antiepileptic Drug Withdrawal in Juvenile Myoclonic Epilepsy." Acta Neurol. Scand. 139: 192-98. https://doi. org/10.1111/ane.13042.

Tang, Xinghua, Peimin Yu, Ding Ding, Yan Ge, Yunbo Shi, Ping Wang, Guoxing Zhu, and Zhen Hong. 2017. "Risk Factors for Seizure Reoccurrence after Withdrawal from Antiepileptic Drugs in Individuals Who Have Been Seizure-Free for over 2 Years." PLos One 12 (8): E0181710.

Téllez-zenteno, José F, Lizbeth Hernández, Nathalie Jette, Jorge G Burneo, Dang Khoa, Elizabeth J Donner, Mark Sadler, et al. 2012. “Discontinuation of Antiepileptic Drugs after Successful Epilepsy Surgery. A Canadian Survey." Epilepsy Res. 102: 23-33. https://doi.org/10.1016/j. eplepsyres.2012.04.018.

Tennison, Michael, Robert Greenwood, Darrell Lewis, and Michael Thorn. 1994. “Discontinuing Antiepileptic Drugs in Children with Epilepsy: A Comparison of a Six-Week and a Nine-Month Taper Period." The New England Journal of Medicine 330: 1407-10.

The International League Against Epilepsy Consortium on Complex Epilepsies. 2018. "Genome-Wide Mega-Analysis Identifies 16 Loci and Highlights Diverse Biological Mechanisms in the Common Epilepsies." Nature Communications 9: 5269. https://doi.org/10.1038/s41467-018-07524-z.

Therneau, Terry M. 2015. "Coxme: Mixed Effects Cox Models." R-Package Description. https://doi. org/10.1111/oik.01149.

Tinuper, P, P Avoni, R Riva, F Provini, E Lugaresi, and A Baruzzi. 1996. "The Prognostic Value of the Electroencephalogram in Antiepileptic Drug Withdrawal in Partial Epilepsies." Neurology 47: 76-78.

Tisi, Jane de, Gail S Bell, Janet L Peacock, Andrew W McEvoy, William F J Harkness, Josemir W Sander, and John S Duncan. 2011. “The Long-Term Outcome of Adult Epilepsy Surgery, Patterns of Seizure Remission, and Relapse: A Cohort Study." The Lancet 378: 1388-95. https://doi.org/10.1016/S01406736(11)60890-8.

Tomson, Torbjörn, Dina Battino, Erminio Bonizzoni, John Craig, Dick Lindhout, Emilio Perucca, Anne Sabers, Sanjeev V Thomas, and Frank Vajda. 2018. "Comparative Risk of Major Congenital Malformations with Eight Different Antiepileptic Drugs: A Prospective Cohort Study of the EURAP Registry." Lancet Neurology 17: 530-38. https://doi.org/10.1016/S1474-4422(18)30107-8.

Tonini, C, E Beghi, A T Berg, G Bogliun, L Giordano, R W Newton, A Tetto, E Vitelli, D Vitezic, and S Wiebe. 2004. "Predictors of Epilepsy Surgery Outcome: A Meta-Analysis." Epilepsy Research 62: 75-87. https://doi.org/10.1016/j.eplepsyres.2004.08.006.

Trinka, Eugen, Gerhard Bauer, Willi Oberaigner, Jean-Pierre Ndayisaba, Klaus Seppi, and Claudia A Granbichler. 2013. “Cause-specific Mortality among Patients with Epilepsy: Results from a 30-year Cohort Study." Epilepsia 54 (3): 495-501.

Trinka, Eugen, Hannah Cock, Dale Hesdorffer, Andrea O Rossetti, Ingrid E Scheffer, Shlomo Shinnar, Simon Shorvon, and Daniel H Lowenstein. 2015. "A Definition and Classification of Status Epilepticus - Report of the ILAE Task Force on Classification of Status Epilepticus." Epilepsia 56 (10): 1515-23. https://doi.org/10.1111/epi.13121.

Tversky, Amos, and Daniel Kahneman. 1981. "The Framing of Decisions and the Psychology of Choice." 
Science 211 (4481): 453-58.

Uesugi, Hideji, T Kojima, M Miyasaka, M Matsuura, T Ohtaka, M Moriiwa, and Y Shimazono. 1994.

"Discontinuation of Antiepileptic Drug Treatment in Controlled Seizure Patients." J Epilepsy 7: $178-88$.

Uijl, Sabine G, Frans S S Leijten, Karel G M Moons, Ellen P H M Veltman, Cyrille H Ferrier, and Cees A Van Donselaar. 2012. "Epilepsy Surgery Can Help Many More Adult Patients with Intractable Seizures." Epilepsy Research 101 (3): 210-16. https://doi.org/10.1016/j.eplepsyres.2012.04.002.

Vân Phan, Thanh, Dirk Smeets, Joel B Talcott, and Maaike Vandermosten. 2018. “Developmental Cognitive Neuroscience Processing of Structural Neuroimaging Data in Young Children: Bridging the Gap between Current Practice and State-of-the-Art Methods." Developmental Cognitive Neuroscience 33: 206-23. https://doi.org/10.1016/j.dcn.2017.08.009.

Vergouw, David, Martijn W. Heymans, Daniëlle a W M Van Der Windt, Nadine E. Foster, Kate M. Dunn, Henriette E. Van Der Horst, and Henrica CW De Vet. 2012. “Missing Data and Imputation: A Practical Illustration in a Prognostic Study on Low Back Pain." Journal of Manipulative and Physiological Therapeutics 35 (6): 464-71. https://doi.org/10.1016/j.jmpt.2012.07.002.

Vermeulen, Lynn, Johannes Van Loon, Tom Theys, Jan Goffin, Kathleen Porke, Koen van Laere, Karolien Goffin, Mathieu Vandenbulcke, Vincent Thijs, and Wim van Paesschen. 2016. "Outcome after Epilepsy Surgery at the University Hospitals 1998-2012." Acta Neurologica Belgica 116 (3): 271-78. https://doi.org/10.1007/s13760-016-0605-6.

Verrotti, A, C D'Egidio, S Agostinelli, P Parisi, A Spalice, F Chiarelli, and G Coppola. 2012. "Antiepileptic Drug Withdrawal in Childhood Epilepsy: What Are the Risk Factors Associated with Seizure Relapse?" European Journal of Paediatric Neurology 16: 599-604. https://doi.org/10.1016/j. ejpn.2012.02.002.

Verrotti, Alberto, S Morresi, F Basciani, R Cutarella, G Morgese, and F Chiarelli. 2000. “Discontinuation of Anticonvulsant Therapy in Children with Partial Epilepsy." Neurology 55: 1393-95.

Verrotti, Alberto, S Morresi, R Cutarella, G Morgese, and F Chiarelli. 2000. "Predictive Value of EEG Monitoring during Drug Withdrawal in Children with Cryptogenic Partial Epilepsy." Neurophysiol Clin 30 (4): 240-45.

Verrotti, Alberto, A Scaparrotta, M Cofini, F Chiarelli, and G M Tiboni. 2014. “Developmental Neurotoxicity and Anticonvulsant Drugs: A Possible Link." Reproductive Toxicology 48: 72-80. https://doi. org/10.1016/j.reprotox.2014.04.005.

Verrotti, Alberto, Daniela Trotta, Carmela Salladini, Guido Morgese, and Francesco Chiarelli. 2003. “Risk Factors for Recurrence of Epilepsy and Withdrawal of Antiepileptic Therapy: A Practical Approach." Annals of Medicine 35: 207-15. https://doi.org/10.1080/07853890310008260.

Vezzani, Annamaria, Robert S Fujinami, H Steve White, Pierre Marie, Ingmar Blümcke, Josemir W Sander, and Wolfgang Löscher. 2016. "Infections, Inflammation and Epilepsy." Acta Neuropathologica 131 (2): 211-34. https://doi.org/10.1007/s00401-015-1481-5.

Vurucu, Sebahattin, Mehmet Saldir, Bulent Unay, and Ridvan Akin. 2010. "Determination of Risk Factors Associated with Seizure Relapse after Antiepileptic Drug Withdrawal." Central European Journal of Medicine 5 (2): 251-56. https://doi.org/10.2478/s11536-009-0049-y.

Webb, Sara J, Christopher S Monk, and Charles A Nelson. 2001. “Mechanisms of Postnatal Neurobiological Development: Implications for Human Development." Developmental Neuropsychology 19 (2): 147-71. https://doi.org/10.1207/S15326942DN1902.

Webster, Michelle. 2017. "Similarities and Differences in the Meanings Children and Their Parents Attach to Epilepsy Medications." Social Science \& Medicine 177: 190-97. https://doi.org/10.1016/j. socscimed.2017.01.065.

Wellmer, J, Ferdinand von der Groeben, Ute Klarmann, Christian Weber, Christian E Elger, Horst Urbach, Hans Clusmann, and Marec von Lehe. 2012." Risks and Benefits of Invasive Epilepsy Surgery Workup with Implanted Subdural and Depth Electrodes." Epilepsia 53 (8): 1322-32. https://doi. org/10.1111/j.1528-1167.2012.03545.x.

West, S, SJ Nolan, J Cotton, S Gandhi, J Weston, A Sudan, R Ramirez, and R Newton. 2015. “Surgery for Epilepsy." Cochrane Database of Systematic Reviews Issue 7: CD010541. https://doi. org/10.1002/14651858.CD010541.pub2.

Whelan, Christopher D, Andre Altmann, Juan A Botıa, Neda Jahanshad, Derrek P Hibar, Julie Absil, Saud 
Alhusaini, et al. 2018. "Structural Brain Abnormalities in the Common Epilepsies Assessed in a Worldwide ENIGMA Study." Brain 141: 391-408. https://doi.org/10.1093/brain/awx341.

Whitehead, J. 1997. The Design and Analysis of Sequential Clinical Trials. 2nd ed. Chichester: Wiley.

Wieser, H G, W T Blume, D Fish, E Goldensohn, A Hufnagel, D King, M R Sperling, and H Lüders. 2001. "Proposal for a New Classification of Outcome with Respect to Epileptic Seizures Following Epilepsy Surgery." Epilepsia 42 (2): 282-86.

Wieser, H G, M Ortega, A Friedman, and Y Yonekawa. 2003. “Long-Term Seizure Outcomes Following Amygdalohippocampectomy." J Neurosurg 98: 751-63.

Wolf, Peter. 2016. “Remission of Epilepsy as a Function of Time." Epilepsy \& Behavior 61: 46-50. https://doi. org/10.1016/j.yebeh.2016.04.043.

World Health Organization. 2006. "Neurological Disorders: Public Health Challenges." Geneva.

Wyllie, E, DK Lachhwani, A Gupta, A CHirla, G Cosmo, S Worley, P Kotagal, P Ruggieri, and WE Bingaman. 2007. "Successful Surgery for Epilepsy due to Early Brain Lesions despite Generalized EEG Findings." Neurology 69: 389-97.

Yang, Lian-hong, Long-yuan Jiang, Jian-qiang Zhong, Shu-qiong Liu, En-xiang Tao, and Jian-hong Ye. 2013. "Correlation between the Changes in Ambulatory Electroencephalography Findings and Epilepsy Recurrence after Medication Withdrawal among the Population in Southern China." Neurol Med Chir (Tokyo) 53: 12-16.

Yardi, Ruta, Anna Irwin, Husam Kayyali, Ajay Gupta, Dileep Nair, Jorge Gonzalez-Martinez, William Bingaman, Imad M Najm, and Lara E Jehi. 2014. “Reducing versus Stopping Antiepileptic Medications after Temporal Lobe Surgery." Annals of Clinical and Translational Neurology 1 (2): 115-23. https://doi.org/10.1002/acn3.35.

Yasiry, Zeid, and Simon D Shorvon. 2012. "How Phenobarbital Revolutionized Epilepsy Therapy: The Story of Phenobarbital Therapy in Epilepsy in the Last 100 Years." Epilepsia 53 (Suppl. 8): 26-39. https://doi.org/10.1111/epi.12026.

Zeng, Tian-fang, Dong-mei An, Jin-mei Li, Yao-hua Li, Lei Chen, Zhen Hong, Ding Lei, and Dong Zhou. 2012. "Evaluation of Different Antiepileptic Drug Strategies in Medically Refractory Epilepsy Patients Following Epilepsy Surgery." Epilepsy Research 101: 14-21. https://doi.org/10.1016/j. eplepsyres.2012.02.013.

Zhang, Le, Xin-yue Jiang, Dong Zhou, Heng Zhang, Shi-min Bao, and Jin-mei Li. 2018. “Postoperative Seizure Outcome and Timing Interval to Start Antiepileptic Drug Withdrawal: A Retrospective Observational Study of Non-Neoplastic Drug Resistant Epilepsy." Scientific Reports 8: 13782. https://doi.org/10.1038/s41598-018-31092-3. 


\section{SUMMARY}

Epilepsy is a disorder characterized by recurrent epileptic seizures. Medications, so called antiepileptic drugs (AEDs), are successful in preventing seizures for the majority of patients. The introduction of this book, Chapter 1, describes the general aspects of epilepsy and its treatment, to continue with the context of this thesis: AED withdrawal. When a person with epilepsy is seizure free, antiepileptic medications can be stopped in some cases without seizures returning. Because of the side-effects profile of most AEDs, the benefits of AED withdrawal are clear, but these are opposed by the risk of seizure recurrence its consequences. This thesis discusses two different patient populations. In Part l, patients who were seizure free on AED treatment alone are discussed, after which Part II focuses on those who were seizure free after epilepsy surgery.

\section{PART I. AED withdrawal in patients without surgery.}

Chapter 2 presents a systematic review with two aims: (1) to meta-analyze the risk of seizure recurrence after AED withdrawal based on all available literature, for both medically treated and surgically treated patients; and (2) to systematically gather information on predictors of seizure recurrence after AED withdrawal. Based on nearly 3700 patients from 26 publications, the average seizure recurrence risk 3-4 years after AED withdrawal was 34\% (95\% confidence interval 28-40\%) for those seizure free on AEDs patients. For patients seizure free after epilepsy surgery, the average recurrence risk in the first 3-4 years was $24 \%$ ( $95 \%$ confidence interval 13-42\%), based on 1025 patients from 5 studies. The number of predictors reported in the literature of outcome after AED withdrawal was 25 for medication-only, and 12 for after epilepsy surgery. Because of the heterogeneity between study designs it was not possible to state which predictors were most important.

In Chapter 3 we investigated the potential cumulative effects of AEDs on the intelligence quotient (IQ) of children. Fifty nine children with focal epilepsy were included. These children had an MRI and neuropsychological investigations available between the ages of 5 and 12 . Linear regression analysis revealed a negative association between the cumulative drug load and IQ. Drug load was defined in drug-years until neuropsychological investigation. After correcting for confounders in multivariable linear regression, the negative association remained with a regression coefficient of -1.2 IQ-points per drug-year (95\% confidence interval: -2.1 to -0.3$)$. There are limitations to the study design, so causal inferences are not possible. These findings do highlight the need for further studies on this topic. 
After the systematic review of predictors in Chapter 2 we conducted an independent participant data meta-analysis in Chapter 4. With this analysis we wanted to determine which predictors are strongest to assess the risks, and to combine these into a prediction model. Ten datasets from previously published cohorts were combined to form one large cohort of 1769 patients. These were analyzed with proportional hazards regression resulting in two prediction models. The first predicts the risk of seizure recurrence after two and five years, and included the following eight predictors: the duration of epilepsy before remission, the seizurefree interval before initiating AED withdrawal, the age at onset of seizures, a history of febrile seizures, the number of seizures before remission, the absence of an epilepsy syndrome with a benign course (childhood absence epilepsy, Rolandic epilepsy or Panayiotopoulos syndrome), developmental delay, and epileptiform abnormalities on an EEG prior to AED withdrawal. The second prediction model predicts the presence of seizures at 10 years after withdrawal. The model contains the following eight predictors: the duration of epilepsy before remission, the seizure-free interval before initiating AED withdrawal, the number of AEDs before withdrawal, female sex, a family history of epilepsy, the number of seizures before remission, the presence of focal seizures, and epileptiform abnormalities on an EEG prior to AED withdrawal.

\section{PART II. AED withdrawal after epilepsy surgery.}

Epilepsy surgery mainly focusses on completely removing the source of epileptic seizures, and by doing so curing the patient from epilepsy. When this is not possible, palliative surgery focusses on relief from symptoms, which is not the focus of this thesis. After successful curative surgery people usually continue with AED treatment for some time, until it is deemed safe to withdraw the medication. The policies and practices regarding the timing of AED withdrawal have changed over time, as this part of the thesis will show.

In 1990 a national program was started in the Netherlands for epilepsy surgery in children. Chapter 5 presents outcomes of the first two decades of pediatric epilepsy surgery in the Netherlands. Between 1990 and 2011, 234 children underwent epilepsy surgery with curative intent. Two years after epilepsy surgery, $74 \%$ of patients were completely seizure free. When comparing the outcomes of 1990-2000 and 2001-2011, there was no statistical difference regarding seizure outcome between these epochs. In the second epoch (2001-2011), however, the amount of children who had completely stopped antiepileptic drug treatment more than doubled: $32 \%$ in $2001-2011$ versus $13 \%$ in 1990-2000.

Chapter 6 investigated epilepsy surgery outcome on a larger scale. We summarized outcomes in relation to histopathological diagnosis in a European cohort of 9147 adults and children operated between 2000 and 2012. There was a difference in seizure outcomes between different 
classes of histopathology, with seizure freedom at two years in $72-76 \%$ of patients with a tumor, vascular malformation or hippocampal sclerosis, and $54-60 \%$ of those with malformation of cortical development, encephalitis, glial scar, and those with no histopathological lesion. Medication freedom increased between one and five years after surgery, from 3-9\% of patients (depending on histopathology) to $17-43 \%$. There was a large difference between the centers regarding the proportion of medication free patients. Lastly, children were more often free from medication at five years post-surgery (45\%) than adults (28\%).

To study the cognitive benefits of early postoperative AED withdrawal in children, the TimeToStop trial was designed, of which Chapter $\mathbf{7}$ contains the study protocol. This randomized clinical trial was designed to allocate children to early withdrawal at four months after surgery, or late withdrawal at twelve months. Primary outcome was cognitive performance, especially executive functions as measured with the EpiTrack Junior, at one and two years after surgery. A secondary objective was to confirm safety of early withdrawal. The study was designed as a multicenter, international trial to be executed in the UK, Germany, France, Switzerland and the Netherlands.

The TimeToStop trial was unsuccessful in recruiting patients, because almost all parents demanded early withdrawal. This was the motivation to start a survey among pediatric epileptologists to uncover current practices of AED withdrawal. The results of this survey are described in Chapter 8. AED withdrawal was started between three and six months on average in case of poly, and between five and eleven months in case of monotherapy. In addition, 46$67 \%$ of respondents stated it was not justified to wait twelve months before starting AED withdrawal. These results confirmed what we suspected: medication is withdrawn early as standard practice in many places already, late withdrawal is not accepted anymore as good option, and therefore the TimeToStop trial was justifiably terminated.

Chapter 9 showcases the development of another prediction tool; this time predicting seizure outcomes after AED withdrawal in the context of pediatric epilepsy surgery. Based on a cohort of 766 European children, prediction models were created analogous to those in Chapter 4. These predict the risk of seizure recurrence at two and five years after the start of AED withdrawal, and the chance of seizure freedom at six years. Factors included in the first model were the age at AED withdrawal, the time to starting AED withdrawal in months since surgery, the presence of multifocal lesions on preoperative MRI, interictal epileptiform discharges on a postoperative EEG, and incomplete resection of the anatomical lesion. Three factors were related to long-term seizure freedom (at six years) and were included in the second model: the average seizure frequency before surgery, the number of AEDs before surgery, and incomplete resection of the anatomical lesion. 
Finally, the general discussion in Chapter $\mathbf{1 0}$ places the main findings in context of the literature. To summarize the findings: for seizure-free patients on medication, and for children after epilepsy surgery, prediction models are presented. These models allow for individual estimation of seizure recurrence risk after AED withdrawal. The models are used in practice to counsel patients, and available on www.epilepsypredictiontools.info. Also in this thesis, the relation between AED treatment in children and their intelligence quotient was studied: AEDs may have a negative impact on cognitive development of children. Finally, we learned several aspects about postoperative medication policy: in the Netherlands, children stopped with medication earlier in later years; children more often stop AEDs after surgery than adults; there is large variation between centers regarding policy; and the average pedicatric epileptologist starts postoperative AED withdrawal within the first year after surgery. 


\section{SAMENVATTING}

Epilepsie is een aandoening gekenmerkt door epileptische aanvallen. Medicatie, zogenaamde anti-epileptica (antiepileptic drugs, AEDs), kunnen bij veel mensen de aanvallen voorkomen. De introductie, Hoofdstuk 1, beschrijft de algemene aspecten van epilepsie en de behandeling, en daarna de context van dit proefschrift: het stoppen van AEDs. Na een tijd zonder aanvallen is het namelijk de vraag of deze medicatie nog nodig is; soms kan deze gestopt worden zonder dat de aanvallen terug komen. AEDs hebben veel bijwerkingen, zo kunnen ze bijvoorbeeld iemand slaperig of minder alert maken en kunnen ze voor geboortedefecten zorgen. Het voordeel van het staken van de medicamenteuze behandeling is duidelijk, maar het risico dat de aanvallen terugkomen met alle gevolgen van dien blijft.

In dit proefschrift worden twee verschillende groepen mensen besproken. DEEL I kijkt naar mensen die onder behandeling van deze medicatie aanvalsvrij zijn geworden. In DEEL II wordt gekeken naar mensen die dankzij een speciale hersenoperatie geen aanvallen meer hebben: epilepsiechirurgie.

\section{DEEL I. Het staken van AEDs bij patiënten die geen epilepsiechirurgie hebben gehad.}

Hoofdstuk 2 heeft alle gepubliceerde artikelen over het staken van AEDs onderzocht met twee doelen: (1) het gemiddelde risico op een aanvalsrecidief te berekenen na het staken van AEDs, zowel voor medicamenteus als chirurgisch behandelde mensen; en (2) op een systematische wijze informatie te verzamelen over mogelijke voorspellers van een aanvalsrecidief na het staken van AEDs. De gemiddelde kans dat aanvallen in de eerste 3-4 jaar terugkomen was $34 \%$ (95\% betrouwbaarheidsinterval $28-40 \%$ ) in het geval van medicamenteuze behandeling, gebaseerd op bijna 3700 patiënten uit 26 verschillende artikelen. was. In het geval van epilepsiechirurgie was de gemiddelde kans dat de aanvallen terug kwamen na het staken van AEDs 24\% (95\% betrouwbaarheidsinterval 13-42\%), gebaseerd op 1025 patiënten uit 5 studies. Er werden 25 verschillende voorspellers gevonden in de literatuur die een aanvalsrecidief zouden voorspellen na het staken van AEDs. In het geval van aanvalsvrijheid na epilepsiechirurgie waren dat er nog eens 12. Er waren echter te veel verschillen tussen de onderzoeksmethoden, het formaat van de studies en de kwaliteit, dat er niet gezegd kon worden welke van deze voorspellers het belangrijkst zijn.

In Hoofdstuk 3 hebben we gekeken naar de mogelijke effecten van AEDs op de intelligentie quotiënt (IQ) van kinderen. Gegevens waren beschikbaar van 57 kinderen, met onder andere een $\mathrm{MRI}$ en een neuropsychologisch onderzoek tussen de leeftijden 5 en 12 jaar. Lineaire 
regressie toonde een negatieve associatie aan tussen cumulatieve AED load en IQ. Cumulatieve AED load wil zeggen, hoe langer en hoe meer medicijnen, des te zwaarder de AED load, en hoe lager het IQ. Na correctie voor confounders bleef deze negatieve associatie met een regressie coëfficiënt van -1.2 IQ punten per AED jaar (95\% betrouwbaarheidsinterval: -2.1 tot -0.3). Er zijn beperkingen aan deze studie, dus er kan geen causaal verband worden aangetoond. Wel zetten deze bevindingen aan tot meer onderzoek op dit onderwerp.

$\mathrm{Na}$ het systematisch onderzoek van de literatuur in Hoofdstuk 2 hebben we een independent participant data meta-analyse uitgevoerd in Hoofdstuk 4. Hiermee wilden we bepalen welke voorspellers het belangrijkst zijn om risico's in te schatten, om deze vervolgens te combineren in een voorspelmodel. We konden tien datasets van eerder uitgevoerd onderzoek combineren tot een grote dataset van 1769 patiënten. Deze gegevens hebben we geanalyseerd met proportional hazards regressie om twee voorspelmodellen te maken.

Het eerste model voorspelt het risico dat de aanvallen terugkomen binnen twee en vijf jaar. Het bevat acht voorspellers: de duur van epilepsie voordat de persoon aanvalsvrij werd, het aanvalsvrije interval tot aan het staken van medicatie, de leeftijd van de eerste aanval, een voorgeschiedenis van koortsconvulsies, het totaal aantal aanvallen voor remissie, de afwezigheid van een epilepsiesyndroom met een bekend gunstig beloop (absence epilepsie op de kinderleeftijd, benigne Rolandische epilepsie, Panayiotopoulos syndroom), ontwikkelingsachterstand, en epileptiforme afwijkingen op een EEG (hersenfilmpje) voorafgaand aan het staken van AEDs.

Het tweede model voorspelt de aanwezigheid van aanvallen 10 jaar na het staken. Het model bevat acht voorspellers: de duur van epilepsie voordat de persoon aanvalsvrij werd, het aanvalsvrije interval tot aan het staken van medicatie, het aantal AEDs voorafgaand aan AED withdrawal, vrouwelijk geslacht, een familiegeschiedenis van epilepsie, het totaal aantal aanvallen voor remissie, de aanwezigheid van focale aanvallen, en epileptiforme afwijkingen op een EEG (hersenfilmpje) voorafgaand aan het staken van AEDs.

\section{DEEL II. Het staken van AEDs na epilepsiechirurgie.}

Epilepsiechirurgie heeft als grootste doel om de oorzaak van epileptische aanvallen volledig weg te nemen, en dus de epilepsie te genezen. Dit is niet altijd mogelijk, in dat geval wordt er gesproken over palliatieve chirurgie (het verlichten van symptomen), wat verder niet ter sprake komt in dit proefschrift. Na een succesvolle -in opzet curatieve- operatie blijven mensen voor een tijd medicatie gebruiken, totdat het veilig wordt geacht om deze te staken. Het beleid met betrekking tot de timing hiervan is onderhevig aan veranderingen naarmate er meer ervaring en data beschikbaar is, zoals uit onderstaande hoofdstukken ook duidelijk wordt. 
In 1990 is men in Nederland begonnen met epilepsiechirurgie voor kinderen. Hoofdstuk $\mathbf{5}$ presenteert de uitkomsten van de eerste 22 jaar epilepsiechirurgie bij kinderen in Nederland. Tussen 1990 en 2011 zijn er 234 kinderen geopereerd met curatieve opzet. Twee jaar na de operatie waren $74 \%$ van de patiënten compleet aanvalsvrij. Een vergelijking tussen de kinderen geopereerd in 1990-2000 en 2001-2011 liet zien dat er geen verandering was in de aanvalsvrijheid. Wel werd de medicatie in het tweede tijdvak vaker gestaakt in de eerste twee jaar: 32\% nam geen medicatie meer, in vergelijking met 13\% in de 1990-2000 groep.

Hoofdstuk 6 heeft naar de uitkomsten van epilepsiechirurgie gekeken op een grotere schaal. We hebben de uitkomsten samengevat in relatie tot de histopathologische diagnoses, in een Europees cohort van 9147 volwassenen en kinderen die tussen 2000 en 2012 geopereerd zijn. De verschillende pathologische diagnosen hadden verschillende uitkomsten van de operatie, met $72 \%-76 \%$ aanvalsvrijheid voor mensen met een tumor, vasculaire malformatie of hippocampale sclerose, en 54-60\% voor mensen met een aanlegstoornis van de hersenschors, encefalitis, een gliaal litteken, en degenen waarbij er geen histopathologische diagnose gesteld werd vanwege normaal weefsel. Afhankelijk van de histopathologische diagnose was er een klein verschil (3-9\%) of groter verschil (17-43\%) in het aantal patiënten welke medicatie stopte tussen één en vijf jaar na de operatie. Er waren grote verschillen tussen de centra met betrekking tot het percentage patiënten zonder AEDs na vijf jaar. Kinderen waren vaker zonder medicatie op vijf jaar na chirurgie (45\%) dan volwassenen (28\%).

Om te kijken naar de mogelijke cognitieve voordelen van vroeg staken van AEDs na epilepsiechirurgie bij kinderen, hebben we de TimeToStop trial ontworpen. Hoofdstuk 7 toont hier het studieprotocol van. Deze gerandomiseerde studie was ontworpen zodat een vergelijking gemaakt kon worden tussen kinderen die vroeg, vier maanden na de operatie, of laat, twaalf maanden na operatie, zouden beginnen met het verminderen van medicatie. De primaire uitkomst zou gemeten worden één en twee jaar na operatie, aan de hand van cognitieve prestaties gemeten met EpiTrack Junior. Daarnaast wilden we ook de veiligheid van vroege medicatie onttrekking bevestigen. Deze trial was ontworpen als een multicenter, internationale trial voor het Verenigd Koninkrijk, Duitsland, Frankrijk, Switzerland en Nederland.

De TimeToStop trial is voortijdig gestaakt omdat geen van de ouders mee wilden doen: ze wensten bijna allemaal vroeg na de operatie te stoppen met de medicatie. Dit was voor ons de aanleiding om een enquête te houden onder kinderepileptologen en hen te vragen naar hun huidig beleid ten opzichte van postoperatief medicatiegebruik. De resultaten van deze enquête zijn beschreven in Hoofdstuk 8. Deze experts gaven aan gemiddeld na drie tot zes maanden te beginnen met het verlagen van de AED dosis als de patiënt meerdere middelen gebruikt. In het geval van één middel was dit na vijf tot elf maanden. Daarnaast zei ook 46$67 \%$ van de experts dat het niet gerechtvaardigd was om twaalf maanden lang door te gaan 
met de medicatie. Deze resultaten bevestigden wat we reeds vermoedden: medicatie wordt tegenwoordig standaard vroeg na de operatie verminderd, laat staken wordt gezien als minder gunstig, en de TimeToStop trial is dus terecht gestaakt.

Hoofdstuk 9 beschrijft de ontwikkeling van een tweede predictiemodel voor de uitkomsten van het staken van AEDs; deze keer in de context van epilepsiechirurgie bij kinderen. Op basis van een cohort van 766 kinderen uit Europa zijn er twee modellen gemaakt vergelijkbaar met de modellen in Hoofdstuk 4. Ze voorspellen het risico op een aanvalsrecidief op twee en vijf jaar na de start van medicatievermindering, en de kans op aanvalsvrijheid na zes jaar. De voorspellers in het eerste model waren: leeftijd waarop AEDs worden verminderd, de tijd tussen operatie en het starten van AED vermindering, de aanwezigheid van multifocale afwijkingen op het preoperatief MRI, interictale afwijkingen op een postoperatief EEG, en incomplete resectie van de anatomische lesie. Drie factoren waren gerelateerd aan langetermijn aanvalsvrijheid (op zes jaar) en zijn in het tweede model opgenomen: het gemiddeld aantal aanvallen voor de operatie, het aantal AEDs voor de operatie, en incomplete resectie van de anatomische lesie.

Tot slot, in de algemene discussie van Hoofdstuk 10 zijn de hoofdbevindingen van de voorgaande hoofdstukken in de context van literatuur geplaatst. Samengevat: dit proefschrift presenteert voorspelmodellen voor mensen die met AEDs aanvalsvrij zijn geworden, en voor kinderen die epilepsiechirurgie hebben ondergaan. Deze modellen maken het mogelijk om voor de individuele patiënt te berekenen wat het risico is dat de aanvallen terug zullen keren. De modellen worden in de praktijk gebruikt om patiënten voor te lichten, en zijn beschikbaar op www.epilepsypredictiontools.info. Een hoofdstuk heeft gekeken naar de relatie tussen AED gebruik bij kinderen en hun intelligentie quotiënt: AEDs hebben mogelijk een negatieve impact op cognitieve ontwikkeling bij kinderen. Als laatste zijn er meerdere conclusies te trekken met betrekking tot postoperatief medicatiebeleid: kinderen die in latere jaren geopereerd waren stopten eerder met medicatie; kinderartsen beginnen meestal in het eerste jaar met het onttrekken van AEDs; in Europa stoppen kinderen vaker dan volwassenen, en is er ook een grote variatie in beleid tussen de centra. 


\section{LIST OF PUBLICATIONS}

Lamberink, HJ, K Boshuisen, PC Van Rijen, PH Gosselaar, and KPJ Braun. 2015. “Changing Profiles of Pediatric Epilepsy Surgery Candidates over Time: A Nationwide Single-Center Experience from 1990 to 2011." Epilepsia 56 (5): 717-25.

Lamberink, HJ, WM Otte, K Geleijns, and KPJ Braun. 2015. "Antiepileptic Drug Withdrawal in Medically and Surgically Treated Patients: A Meta-Analysis of Seizure Recurrence and Systematic Review of Its Predictors." Epileptic Disorders 17 (3): 211-28.

Boshuisen, K, HJ Lamberink, MM van Schooneveld, JH Cross, A Arzimanoglou, I van der Tweel, K Geleijns, CS Uiterwaal, and KPJ Braun. 2015. “Cognitive Consequences of Early versus Late Antiepileptic Drug Withdrawal after Pediatric Epilepsy Surgery, the TimeToStop (TTS) Trial: Study Protocol for a Randomized Controlled Trial." Trials 16: 482.

Lamberink, HJ, K Boshuisen, PC van Rijen, PH Gosselaar, and KPJ Braun. 2016. “Kinderepilepsiechirurgie in Nederland: Patiëntkarakteristieken En Chirurgische Uitkomsten Tussen 1990 En 2011 [Dubbelpublicatie van Epilepsia 56:171-175]." Tijdschr Neurol Neurochir 117 (3): 110-19.

Lamberink, HJ, WM Otte, AT Geerts, M Pavlovic, J Ramos-Lizana, AG Marson, J Overweg, et al. 2017. "Individualised Prediction Model of Seizure Recurrence and Long-Term Outcomes after Withdrawal of Antiepileptic Drugs in Seizure-Free Patients: A Systematic Review and Individual Participant Data Meta-Analysis." The Lancet Neurology, 16(7):523-531.

Lamberink, HJ, K Boshuisen, WM Otte, K Geleijns, KPJ Braun, M Feucht, G Gröppel, et al. 2018. "Individualized Prediction of Seizure Relapse and Outcomes Following Antiepileptic Drug Withdrawal after Pediatric Epilepsy Surgery." Epilepsia 59 (3).

Lamberink, HJ, Van Eijsden P, Braun KPJ. 2018. "Surgery highly effective for seizure cessation in drug-resistant epilepsy." Journal of Pediatrics 196:324-327.

Otte, WM, JK Tijdink, PL Weerheim, HJ Lamberink, and CH Vinkers. 2018. "Adequate Statistical Power in Clinical Trials Is Associated with the Combination of a Male First Author and a Female Last Author." eLIFE 7: e34412.

Baud, MO, T Perneger, R Attila, MC Pensel, CE Elger, B Rydenhag, K Malmgren, ..., HJ Lamberink, ..., et al. 2018. "European Trends in Epilepsy Surgery." Neurology 91 (2): e96-106.

Lamberink, HJ, WM Otte, MRT Sinke, P Glasziou, JK Tijdink, and CH Vinkers. 2018. "Statistical Power of Clinical Trials Increased While Effect Size Remained Stable: An Empirical Analysis of 136,212 Clinical Trials between 1975 and 2014." Journal of Clinical Epidemiology 102: 12-128.

Lamberink, HJ, K Geleijns, WM Otte, A Arzimanoglou, JH Cross, CM Korff, G Ramantani, and KPJ Braun. 2018. "Why the TimeToStop Trial Failed to Recruit: A Survey on Antiepileptic Drug Withdrawal after Paediatric Epilepsy Surgery." Epileptic Disorders 20 (5): 374-85.

Diessen, E van, HJ Lamberink, WM Otte, N Doornebal, OF Brouwer, FE Jansen, and KPJ Braun. 2018. "A Prediction Model to Determine Childhood Epilepsy after 1 or More Paroxysmal Events." Pediatrics 142 (6): e20180931. 


\section{ABOUT THE AUTHOR}

Herm Lamberink was born on November 30, 1989, in Zwolle, the Netherlands. In 2008 he commenced his BSc at the University College Roosevelt in Middelburg. In 2012 he started SUMMA (Selective Utrecht Medical Master), a medical master with a strong focus on research. From the first year of this program he worked on a research project supervised by Prof. Dr. Kees Braun and Dr. Kim Boshuisen, on outcomes of epilepsy surgery in children, at the Pediatric Neurology department. Research and epilepsy had captured his interest and aside his medical training he stayed involved in various research projects, including taking on responsibility for the coordination of the TimeToStop trial.


After graduating as M.D. in 2016 Herm joined the UMC Utrecht Brain Center as a PhD candidate, supervised by Prof. Dr. Kees Braun, Dr. Wim Otte and Dr. Karin Geleijns. This research lead to, among others, the website www.EpilepsyPredictionTools.info. For the presentation of a prediction tool for AED withdrawal he won an award at the 2017 congress of the European Academy of Neurology. Moreover, together with Kim Boshuisen he won an award by the Dutch Association for Neurology for presenting the Dutch pediatric epilepsy surgery outcomes in 2017. Provided with an opportunity to dive into research ethics and research methodology, Herm took a short break from studying people with epilepsy, working as a researcher on a big data project for a year: 'Predicting Responsible Research', led by Dr. Christiaan Vinkers and Dr. Wim Otte. In this project the quality of 180,000 clinical trials across medical disciplines was reviewed. After this year Herm returned to the Pediatric Neurology department to focus on finishing his $\mathrm{PhD}$ thesis revolving around the withdrawal of antiepileptic drugs. Aside his $\mathrm{PhD}$ defence, he is also looking forward to his wedding with his soon-to-be ${ }^{4}$ wife Anne, both happening this autumn. 


\section{DANKWOORD}

Dat u dit proefschrift nu kunt lezen is alleen maar mogelijk dankzij een groep geweldige mensen om mij heen. Ik heb enorm veel geluk gehad met een sterk en attent team van begeleiders, leuke collega's, goede ondersteuning vanuit het UMC Utrecht en heel veel mensen in mijn omgeving die mij het belang van ontspanning, vrienden en familie telkens weer hebben laten inzien. Ik wil hier iedereen die een bijdrage heeft geleverd, direct of indirect, heel hartelijk bedanken!

Dit proefschrift was niet mogelijk geweest zonder gegevens van duizenden mensen met epilepsie-methen wil ik beginnen.Zonder proefpersonen die meewerken aan wetenschappelijk onderzoek is er geen vooruitgang mogelijk in de zorg.

Kees, toen ik in 2013 als masterstudent bij jou aanklopte voor 'een klein onderzoeksproject voor naast de studie' had ik niet kunnen bedenken dat dit project het begin van een prachtig PhD traject zou zijn. Bedankt voor je grote vertrouwen in mij, voor je enthousiaste en positieve houding, en voor je feilloze gevoel voor onderzoek van de toekomst. Zonder jouw ideeën waren de projecten in dit proefschrift niet ontstaan. Ik vind je energie stimulerend en van jouw kennis heb ik veel kunnen leren.

Karin, jij was net naar Utrecht gekomen toen we bespraken of ik hier misschien aan een promotietraject zou beginnen. "Welkom bij het team", hoor ik je nog zeggen. Jij hebt een scherp oog voor kwaliteit in onderzoek, hoge standaarden, maar hebt aan de andere kant altijd als eerste mijn persoonlijke groei en welzijn voorop gezet. Dat jij je eigen weg durft te gaan en recent een ander pad bent ingeslagen vind ik enorm inspirerend.

Wim, wat een geluk dat ik jou ook als copromotor mag hebben. Dankzij jouw sturing en ervaring heb ik mijn interesse in analysetechnieken en epidemiologie kunnen verwezenlijken met veel kennis, ervaring en enthousiasme. Je bent voor mij het voorbeeld van Wetenschapper: veelzijdig, creatief, met een zeer goede methodologische basis. Niet bang om nieuwe projecten en samenwerkingen aan te gaan, en bovenal, een heel goede en bereikbare begeleider.

Kim, dit proefschrift kun je toch ook een klein beetje als jouw nalatenschap zien; het bouwt voort op ideeën en projecten die zijn ontstaan bij jouw onderzoek. Ik wil je bedanken voor je fijne begeleiding tijdens mijn studentenjaren en de prettige samenwerking. 
Geachte professor Eijkemans, professor Brouwer, en Dr. Leijten, bedankt dat $u$ in de leescommissie van dit proefschrift wilde plaatsnemen en daar tijd en energie in heeft gestoken. Dear professor Arzimanoglou and professor Sander, thank you for your time and energy in taking part in the reading committee for this thesis.

Medeauteurs aan mijn artikelen, C.H. Stevering, Dr. M.M.J. van Schooneveld, Dr. A.T. Geerts, Dr. J. Overweg, Dr. K. Boshuisen, Dr. P.C. van Rijen, Dr. P.H. Gosselaar, Dr. I. van der Tweel, Dr. C.S.P.W. Uiterwaal, bij het schrijven van elk artikel heb ik weer heel veel geleerd van jullie kennis en kunde - dank daarvoor.

Dear co-authors of articles in this thesis, dear J. Woodfield, Dr. M. Pavlovic, Dr. J. Ramos-Lizana, Prof. Dr. A.G. Marson, Dr. L. Sauma, Prof. L.M. Specchio, Prof. M. Tennison, Dr. T.M.O. Cardoso, Prof. Dr. S. Shinnar, Prof. Dr. D. Schmidt, Prof. Dr. Blümcke, Collaborators of the European Epilepsy Brain Bank consortium, Prof. Dr. J.H. Cross, Prof. Dr. A. Arzimanoglou, Dr. C.M. Korff, Dr. G. Ramantani, in writing articles together with you I have benefited a great deal from your knowledge and knowhow - thank you.

Renate, het geeft mij een gerust gevoel dat ik de NeedToStop studie aan jou mocht overdragen. Ik wens je een interessante reis toe met dit onderzoek en andere projecten rondom het verlagen van anti-epileptica. Team NeedToStop, beste Mireille, Charlotte, Manon, Albert, Hilde, Simon, Louis, Lotte, Jack, Elise en Hannah, bedankt voor jullie inzet voor deze studie. We zijn goed onderweg!

Christiaan, Joeri, en Wim weer, jullie ben ik dankbaar voor het opzetten van een geweldig project waar ik een jaar aan mocht werken. Dankzij dit project heb ik heel veel geleerd over de kwaliteit van onderzoek, over epidemiologie, en heb ik ook dit proefschrift mooi kunnen afronden. Lotty, Anneke en Pauline, jullie maakten het team compleet en ik ben blij dat ik met jullie kon samenwerken.

Hélène, het leek wel alsof jij altijd aan het telefoneren was als ik een vraag voor je had. Maar hoe druk je het ook had, altijd had je tijd voor een praatje en gaf mij precies de hulp die ik nodig had.

Eltje, ik heb genoten van jou als kamergenoot, altijd op zoek naar de diepere zin in je werk en leven, volle aandacht voor de patiënt -en zo attent en lief dat je bent voor iedereen! Ook als collega heb ik veel gehad aan je adviezen en hulp met alle praktische onderzoeksvragen.

Tijdens dit project heb ik ook mogen leren van het begeleiden van studenten in hun bachelorof masteronderzoek. Silvia, Carmen, Lennart en Michelle, bedankt voor jullie enthousiasme, harde werk, en goede lessen ook voor mij als begeleider. 
Er zijn veel mensen binnen het UMC Utrecht geweest die belangrijk ondersteunend werk doen. Zonder hen kan een onderzoeksafdeling niet functioneren, dus ik wil hier de datamanagers Leo, Hans, Zimbo en Yvonne bedanken; de kwaliteitscoördinatoren Hanneke en Irene; voor al het juridisch advies Jan; en voor de ondersteuning met betrekking tot financiële zaken Annet en Silvia.

Samen met alle collega-onderzoekers van de kinderneurologie heb ik meer koffie gedronken dan gezond en zoveel kilometers gewandeld achter het WKZ dat het tijd is voor nieuwe schoenen. Bedankt voor de gezellige tijd op de KNF kamer Bart, Hanna en Eltje, de wandelingen en gesprekken Anne, de soepele samenwerking Eric en Maurits, en de mooie afsluitende tijd op de $3^{\text {e }}$ Matea en Lotte. Dan zijn er ook nog collega's van de psychiatrie, KT1PRO, de mooiste kamer die helaas niet meer bestaat; Sonja, Judith, Jalmar, Jasper, Judith, Bart, Martijn en Jessica, ik ben blij dat ik een jaar bij jullie heb mogen vertoeven.

Er zijn vrienden, te veel om op te noemen: mannen van het MAG, zonder de jaarlijkse tocht was ik vast vastgeroest aan mijn bureaustoel. Wat een prachtige traditie. NEXT mag ook niet ontbreken in dit dankwoord, waar ik elke maandagavond mijn muzikale ontspanning vind, en die mij menig zware dinsdagochtend heeft bezorgd. Ook de avonden aan de klimwanden met de Sterksten hebben bijgedragen aan de welkome ontspanning en spierpijn. Al mijn Middelburgse vrienden met veel spelletjesavonden en kampeerweekenden, wat fijn dat wij al zo lang samen op trekken, banden kunnen versterken en bij elkaar terecht kunnen.

Stephanie en Saskia, het is mij een eer dat jullie mijn paranimfen zijn. Met jullie naast mij voel ik mij gesteund en ik weet dat ik bij jullie terecht kan voor eerlijke gesprekken, goede reizen en mooie ervaringen.

Anne en Sjoerd, jullie zijn geweldig, wat fijn dat jullie mij en Anne ook wilden ondersteunen in dat andere grote evenement dit jaar.

Co, Fijke, Jelle, Lieke en Veerle, familie is belangrijk en wat fijn is het dat ik in jullie een geweldige tweede familie er bij heb gekregen.

Kirsten, van je familie moet je het hebben, en dat ik in hetzelfde jaar getuige mocht zijn op de bruiloft van mijn enige zus en dat jij dat ook bij mij bent maakt mij blij. Joachim, de cryptobankprofeet, jij maakt het feest compleet.

Lieve papa en mama, jullie zijn mijn voorbeeld in het uitdragen van je overtuigingen, niet alleen in woorden maar juist ook in daden. En van jullie daden straalt veel liefde uit, bedankt voor dat voorbeeld. 
Lieve, lieve Anne, wat een geluk dat ik jou heb, en jij mij. Tijdens de voorbereidingen voor dit boek heb jij altijd in mij geloofd en mij aangemoedigd. Maar ook heb je oneindig veel geduld gehad. Voor dat geduld, de lieve sturingen en bewaking van de balans in mijn leven ben ik je heel dankbaar. Met jou voel ik mij sterk en gelukkig en ik kijk uit naar alles wat wij samen mogen beleven. 



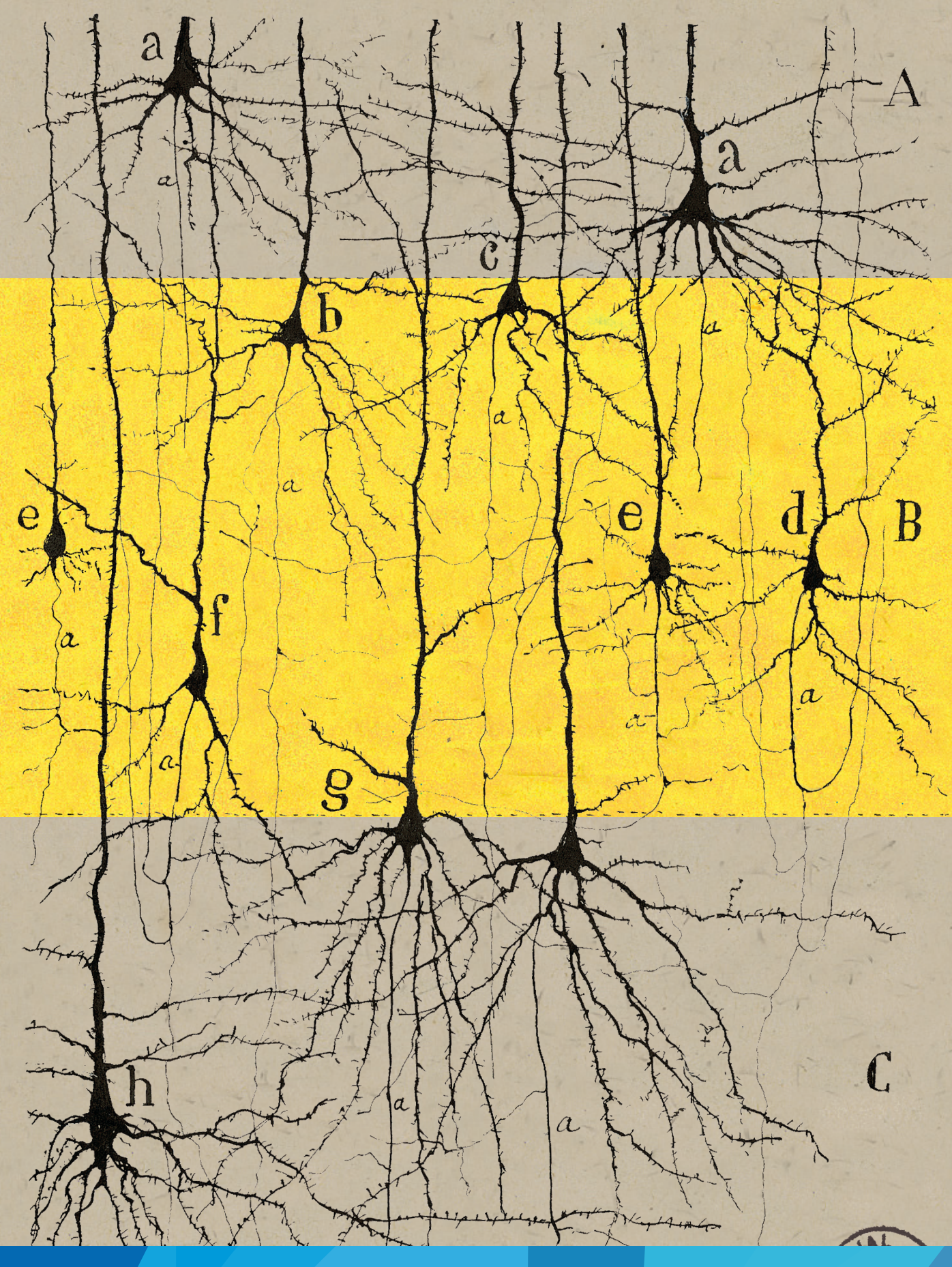

ISBN 978-90-393-7186-2

)$f^{m} x+1$ 